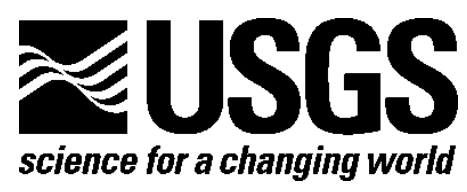

Prepared in cooperation with the University of Tennessee, Louisiana Department of Wildlife and Fisheries, and the U.S. Fish and Wildlife Service

\title{
Population Viability and Connectivity of the Louisiana Black Bear (Ursus americanus luteolus)
}

By Jared S. Laufenberg and Joseph D. Clark

Open-File Report 2014-1228

U.S. Department of the Interior

U.S. Geological Survey 


\section{U.S. Department of the Interior SALLY JEWELL, Secretary}

\section{U.S. Geological Survey \\ Suzette M. Kimball, Acting Director}

U.S. Geological Survey, Reston, Virginia: 2014

For more information on the USGS—-the Federal source for science about the Earth, its natural and living resources, natural hazards, and the environment-visit http://www.usgs.gov or call 1-888-ASK-USGS (1-888-275-8747)

For an overview of USGS information products, including maps, imagery, and publications, visit http://www.usgs.gov/pubprod

To order this and other USGS information products, visit $h$ ttp://store.usgs.gov

Any use of trade, firm, or product names is for descriptive purposes only and does not imply endorsement by the U.S. Government.

Although this information product, for the most part, is in the public domain, it also may contain copyrighted materials as noted in the text. Permission to reproduce copyrighted items must be secured from the copyright owner.

Suggested citation:

Laufenberg, J.S., and Clark, J.D., 2014, Population viability and connectivity of the Louisiana Black Bear (Ursus americanus luteolus): U.S. Geological Survey Open-File Report 2014-1228, 104 p.,

http://dx.doi.org/10.3133/ofr20141228.

ISSN 2331-1258 (online) 


\section{Acknowledgments}

Funding was provided by the Louisiana Department of Wildlife and Fisheries, U.S. Fish and Wildlife Service, U.S. Army Corps of Engineers, Roy O. Martin Timber Company, Black Bear Conservation Coalition, University of Tennessee, and U.S. Geological Survey. Special thanks go to Maria Davidson (Louisiana Department of Wildlife and Fisheries) and Debbie Fuller (U.S. Fish and Wildlife Service) for their logistical and contractual assistance and Terry White (University of Tennessee) who provided administrative support throughout this project. We gratefully acknowledge University of Tennessee Master of Science students M. J. Hooker, C. L. Lowe, J. C. Troxler, and K. C. O’Connell and Louisiana State University Master of Science students J. F. Benson and A. C. Crook who collected much of the data for the study. We wish to thank the many technicians and land owners that contributed to this project for their hard work and hospitality, respectively. R. B. Chandler (University of Georgia) assisted with the capture-mark-recapture modeling, and P. Beier (Northern Arizona University), H. L. Beyer (University of Queensland), M. S. Boyce (University of Alberta), S. Ciuti (University of Freiburg), J. D. Forester (University of Minnesota), R. Greco (U.S. Fish and Wildlife Service), S. L. King (U.S. Geological Survey), B. T. McClintock (National Oceanic and Atmospheric Administration), T. L. McDonald (West, Inc.), A. Saxton (University of Tennessee), and K. A. Zeller (University of Massachusetts) provided valuable assistance with the step-selection function analysis. Thanks also go to D. Paetkau for guidance with population genetics analyses. We are grateful to J. L. Belant (Mississippi State University) and D. L. Garshelis (Minnesota Department of Natural Resources) for providing samples for DNA analysis, to M. J. Chamberlain (University of Georgia) for radio telemetry and reproduction data, and to J. Goad for conducting aerial telemetry. 


\section{Contents}

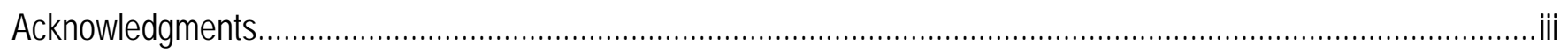

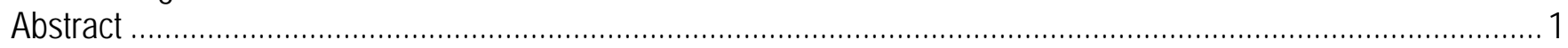

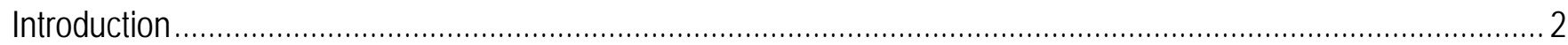

Purpose and Scope

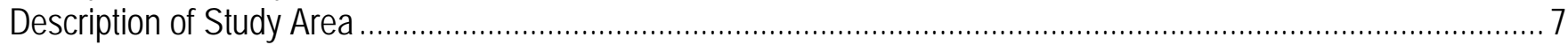

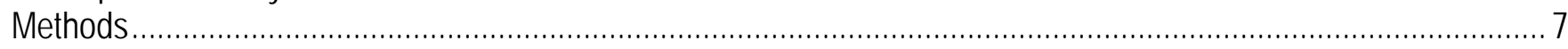

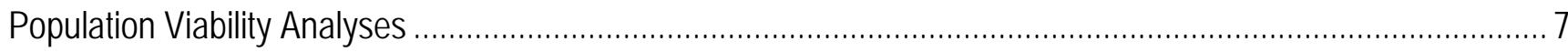

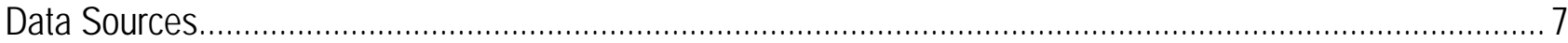

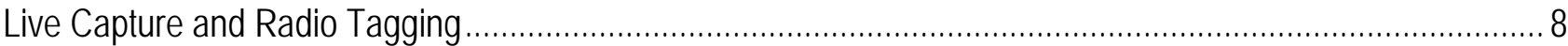

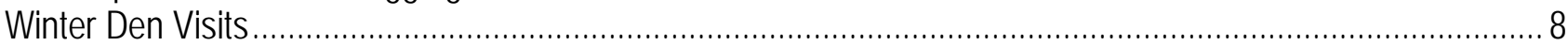

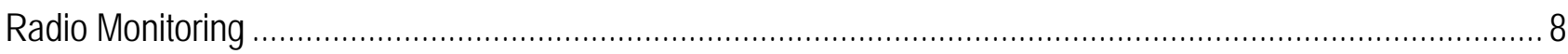

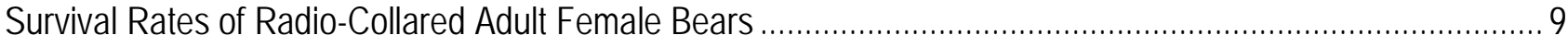

Reproductive Rates of Radio-Collared Adult Female Bears .................................................................... 11

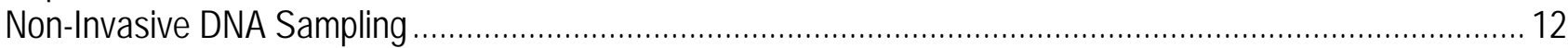

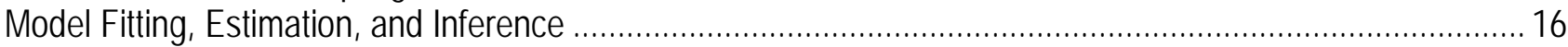

Demographic Rates from the Capture-Mark-Recapture Dataset............................................................ 16

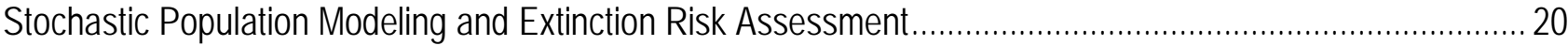

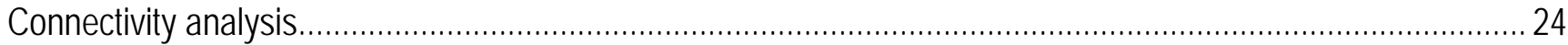

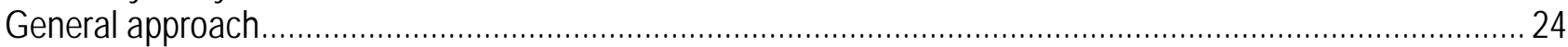

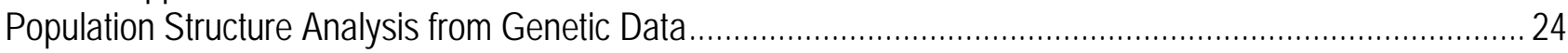

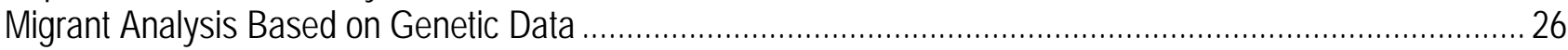

GPS Telemetry Data to Estimate Successful Interchange Rates and Paths ................................................ 27

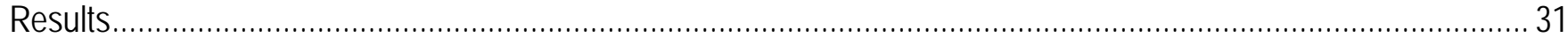

Population Viability Analysis .............................................................................................................. 31

Survival Rates of Radio-Collared Adult Female Bears ……................................................................... 31

Reproductive Rates of Radio-Collared Adult Female Bears .................................................................... 35

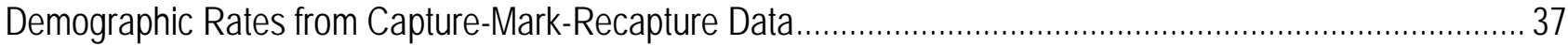

Stochastic Population Modeling and Extinction Risk Assessment............................................................ 45

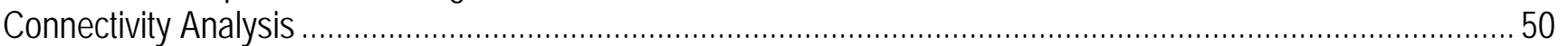

Population Structure Analysis from Genetic Data....................................................................... 50

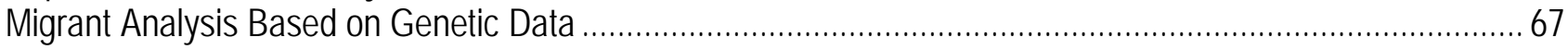

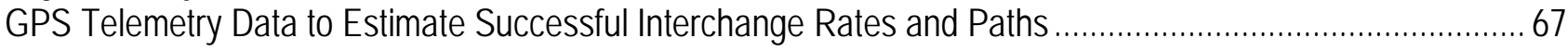

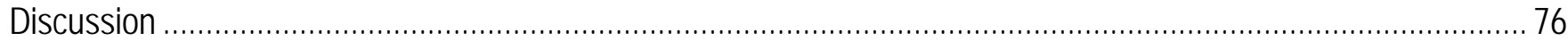

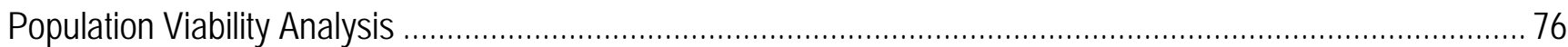

Survival Rates of Radio-Collared Adult Female Bears …………................................................................76

Reproductive Rates of Radio-Collared Adult Female Bears ..................................................................... 77

Demographic Rates from Capture-Mark-Recapture Data.........................................................................

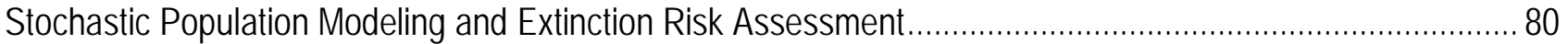

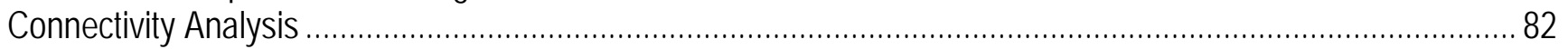

Population Structure and Migrant Analysis .......................................................................................... 82

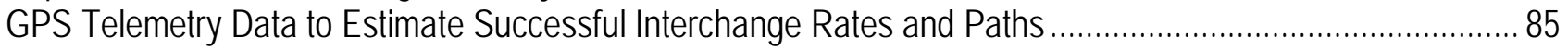

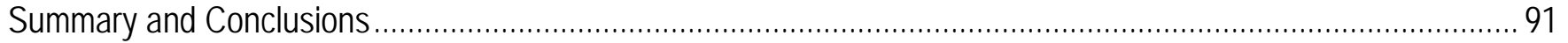

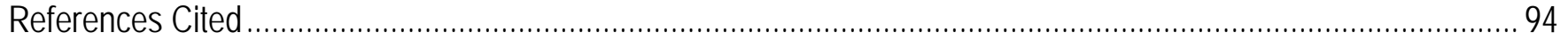




\section{Figures}

1. Map showing the study area showing each of the four subpopulations of Louisiana black bear within the Lower Mississippi Alluvial Valley in Louisiana.

2. Graph showing posterior distributions of assumed-alive and assumed-dead mean annual adult female survival estimates for Louisiana black bears within the Three Rivers Complex and Tensas River Basin in Louisiana, 2002-12.

3. Graph showing annual estimates and 95-percent credible intervals of adult female survival for Louisiana black bears within the Tensas River Basin and Three Rivers Complex in Louisiana, 2002-12.

4. Graph showing annual estimates and 95-percent credible intervals of adult female survival for Louisiana black bears within the Tensas River Basin and Three Rivers Complex in Louisiana, 2002-12.

5. Graph showing posterior distributions for proportions of adult female Louisiana black bears with no litters, cubs, and yearlings within the Tensas River Basin and Three Rivers Complex in Louisiana, 2002-12.

6. Graph showing estimated litter size probabilities of cub and yearling litters and 95-percent credible intervals for adult female Louisiana black bears within the Tensas River Basin and Three Rivers Complex in Louisiana, 2002-12.

7. Graph showing annual number of DNA-based initial captures and recaptures of Louisiana black bears from hair-snare sampling within the Tensas River Basin, Upper Atchafalaya River Basin, and Lower Atchafalaya River Basin in Louisiana, 2006-12

8. Graph showing estimates and 95-percent credible intervals of abundance, population growth, apparent survival, and per-capita recruitment from Model 1 for female Louisiana black bears within the Tensas River Basin in Louisiana, 2006-12.

9. Graph showing estimates and 95-percent credible intervals of abundance, population growth, apparent survival, and per-capita recruitment from Model 2 for female Louisiana black bears within the Tensas River Basin in Louisiana, 2006-12.

10. Graph showing estimates and 95-percent credible intervals of abundance, population growth, apparent survival, and per-capita recruitment from Model 1 for female Louisiana black bears within the Upper Atchafalaya River Basin in Louisiana, 2007-12.

11. Graph showing estimates and 95-percent credible intervals of abundance, population growth, apparent survival, and per-capita recruitment from Model 2 or female Louisiana black bears within the Upper Atchafalaya River Basin in Louisiana, 2007-12.

12. Graph showing estimates and 95-percent credible intervals of abundance, population growth, apparent survival, and per-capita recruitment from Model 1 for female Louisiana black bears within the Lower Atchafalaya River Basin in Louisiana, 2010-12.

13. Graph showing estimates and 95-percent credible intervals of abundance, population growth, apparent survival, and per-capita recruitment from Model 2 for female Louisiana black bears within the Lower Atchafalaya River Basin in Louisiana, 2010-12.

14. Graph showing factorial correspondence analysis results for black bears in Minnesota, Mississippi, the White River Basin in Arkansas, and the Tensas River Basin north of Interstate 20, Tensas River Basin south of Interstate 20, Three Rivers Complex, Upper Atchafalaya River Basin, and Lower Atchafalaya River Basin in Louisiana.

15. Graph showing factorial correspondence analysis results for black bears in Minnesota and the Tensas River Basin north of Interstate 20, Tensas River Basin south of Interstate 20, Upper Atchafalaya River Basin, and Lower Atchafalaya River Basin in Louisiana.... 
16. Graph showing factorial correspondence analysis results for black bears within the Tensas River Basin north of Interstate 20, Tensas River Basin south of Interstate 20, Three Rivers Complex, and Upper Atchafalaya River Basin in Louisiana.

17. Graph showing factorial correspondence analysis results for black bears within the White River Basin in Arkansas and the Tensas River Basin north of Interstate 20 and Tensas River Basin south of Interstate 20 in Louisiana...

18. Graph showing factorial correspondence analysis results for black bears within the Tensas River Basin north of Interstate-20 and south of Interstate-20 in Louisiana.

19. Graph showing factorial correspondence analysis results for black bear cubs born within the Three Rivers Complex in Louisiana

20. Graph showing factorial correspondence analysis results for black bears within the Upper Atchafalaya

River Basin in Louisiana

21. Graph showing factorial correspondence analysis results for bears within the Lower Atchafalaya River Basin in Louisiana...

22. Graph showing mean capture locations from DNA-based captures for black bears in Lower Atchafalaya River Basin, Louisiana, 2010-12. From Troxler 2013.

23. Chart shwoing proportional population ancestries for 556 black bears from Minnesota, western Mississippi, southeast Mississippi, White River Basin in Arkansas, and Tensas River Basin, Three Rivers Complex, Upper Atchafalaya River Basin, and Lower Atchafalaya River Basin in Louisiana, 2006-12......

24. Graph showing program STRUCTURE $\log [K]$ values across 10 chains for each value of $K$ from 2 to 11 , for Louisiana black bears in Louisiana, 2006-12

25. Graph showing estimated $\Delta \log [K]$ values from Program STRUCTURE population clustering analyses for values of $K$ from 3 to 10 for Louisiana black bears in Louisiana, 2006-12.

26. Graph showing proportional population ancestries for black bears within the Tensas River Basin in Louisiana, 2006-12, assigned to populations in Minnesota, White River Basin, Tensas River Basin, Upper Atchafalaya River Basin, or Lower Atchafalaya River Basin

27. Graph showing proportional population ancestries for black bears within the southern portion of the White River Basin in Arkansas, the northern portion of the Tensas River Basin in Louisiana, and northwestern Mississippi, 2006-12, assigned to populations in Minnesota, White River Basin, Tensas River Basin, Upper Atchafalaya River Basin, or Lower Atchafalaya River Basin

28. Graph showing proportional population ancestries for black bears within the Three Rivers Complex in Louisiana, 2006-12, assigned to populations in Minnesota, White River Basin, Tensas River Basin, Upper Atchafalaya River Basin, or Lower Atchafalaya River Basin

29. Graph showing step lengths for female and male Louisiana black bears based on GPS radio collar data collected in Louisiana, during 2010-12.

30. Graph showing turning angles for female and male Louisiana black bears based on GPS radio collar data collected in Louisiana, during 2010-12.

31. Graph showing relation between probability of selection of steps and distance to natural cover by distance to roads, and distance to agriculture for female Louisiana black bears during 2010-12 .............73

32. Map showing cost surface for female bears, Louisiana, 2011-12 …................................................75

33. Map showing cost surface for male bears, Louisiana, 2011-12 …................................................76

34. Map showing relative densities of simulated successful dispersal trajectories for male bears in Louisiana 
35. Map showing relative densities of simulated successful dispersal trajectories for female bears in Louisiana.....

36. Map showing projected movement paths for female Louisiana black bears after the addition of a hypothetical 3,000-meter-wide corridor between the Lower Atchafalaya River Basin and Upper Atchafalaya River Basin subpopulations in Louisiana.

\section{Tables}

1. Distribution of hair-collection sites for study areas in the Tensas River Basin (TRB), Upper Atchafalaya River Basin (UARB), and Lower Atchafalaya River Basin (LARB), Louisiana.

2. Microsatellite markers used for individual identification and population genetics analyses for Louisiana black bears in the Tensas River Basin (TRB), Upper Atchafalaya River Basin (UARB), and Lower Atchafalaya River Basin (LARB), Louisiana, 2006-12.

3. Estimated transition rates between reproductive states for adult female Louisiana black bears in the Tensas River Basin (TRB) and Three Rivers Complex (TRC), Louisiana, 2002-12.

4. Summary of 10,000 simulated population trajectories over a 100 -year period for female Louisiana black bears in the Tensas River Basin (TRB) and Upper Atchafalaya River Basin (UARB), Louisiana. .....46

5. Summary of 10,000 simulated population trajectories over a 100-year period for female Louisiana black bears in the Three Rivers Complex (TRC), Louisiana.

6. Summary of 10,000 simulated population trajectories over a 100-year period for female Louisiana black bears in the Three Rivers Complex, Louisiana, USA.

7. Global Positioning System (GPS) collars deployed on bears in Louisiana, 2010-12.

8. Cox proportional hazards models used to create step-selection functions, Louisiana, 2010-12.

9. Number of successful dispersal paths between four black bear subpopulations in Louisiana, 2010-12, with and without the addition of hypothetical corridors and non-permanently protected natural land cover.

10. Estimates of annual survival for adult female black bears from unhunted populations within the southeastern United States. 


\section{Conversion Factors}

International System of Units to Inch/Pound

\begin{tabular}{|c|c|c|}
\hline Multiply & By & To obtain \\
\hline \multicolumn{3}{|c|}{ Length } \\
\hline centimeter (cm) & 0.3937 & inch (in.) \\
\hline meter (m) & 3.281 & foot (ft) \\
\hline kilometer (km) & 0.6214 & mile (mi) \\
\hline \multicolumn{3}{|c|}{ Area } \\
\hline square meter $\left(\mathrm{m}^{2}\right)$ & 10.76 & square foot $\left(\mathrm{ft}^{2}\right)$ \\
\hline square kilometer $\left(\mathrm{km}^{2}\right)$ & 0.3861 & square mile $\left(\mathrm{mi}^{2}\right)$ \\
\hline \multicolumn{3}{|c|}{ Volume } \\
\hline cubic meter $\left(\mathrm{m}^{3}\right)$ & 264.2 & gallon (gal) \\
\hline cubic centimeter $\left(\mathrm{cm}^{3}\right)$ & 0.06102 & cubic inch $\left(\mathrm{in}^{3}\right)$ \\
\hline \multicolumn{3}{|c|}{ Mass } \\
\hline gram (g) & 0.03527 & ounce, avoirdupois (oz) \\
\hline kilogram (kg) & 2.205 & pound avoirdupois (lb) \\
\hline
\end{tabular}

\section{Datum}

Vertical coordinate information is referenced to the"National Geodetic Vertical Datum of 1929 (NGVD 29)."

Horizontal coordinate information is referenced to the North American Datum of 1983 (NAD 83)].

Altitude, as used in this report, refers to distance above the vertical datum. Negative altitude refers to distance below the vertical datum. 


\title{
Population Viability and Connectivity of the Louisiana Black Bear (Ursus americanus luteolus)
}

\author{
By Jared S. Laufenberg ${ }^{1}$ and Joseph D. Clark ${ }^{2}$
}

\begin{abstract}
In 1992, the U.S. Fish and Wildlife Service (USFWS) granted Ursus americanus luteolus (Louisiana black bear) threatened status under the U.S. Endangered Species Act of 1973, listing loss and fragmentation of habitat as the primary threats. A study was developed by the U.S. Geological Survey in cooperation with the University of Tennessee, the Louisiana Department of Wildlife and Fisheries, and the USFWS to estimate demographic rates and genetic structure of Louisiana black bear populations; evaluate relations between environmental and anthropogenic factors and demographic, genetic, and movement characteristics of Louisiana black bear populations; and develop data-driven stochastic population projection models to assess long-term persistence of individual subpopulations and the overall black bear population in Louisiana.

Data were collected with non-invasive DNA sampling, live capture, winter den visits, and radio monitoring from 2002 to 2012 in the four areas supporting breeding subpopulations in Louisiana: Tensas River Basin (TRB), Upper Atchafalaya River Basin (UARB), Lower Atchafalaya River Basin (LARB), and Three Rivers Complex (TRC). Bears were live trapped and radio collared in the TRB and TRC to estimate survival and reproductive rates, deterministic matrix models were used to estimate asymptotic growth rates, and stochastic population models were used to estimate long-term viability. DNA extracted from hair collected at baited, barbedwire enclosures in the TRB, UARB, and LARB and capture-mark-recapture (CMR) analysis based on Bayesian hierarchical modeling methods were used to estimate apparent survival $(\phi)$, per capita recruitment $(\gamma)$, abundance $(N)$, realized growth rate $(\lambda)$, and long-term viability.

From 2002 to 2012, we radio monitored 86 adult females greater than $(>) 2$ years old within the TRB, and 43 adult females were monitored in the TRC. The mean annual survival rate estimate ranged from 0.97 to 0.99 for the TRB and from 0.93 to 0.97 for the TRC. Fecundity and yearling recruitment in the TRB were 0.47 and 0.15 , respectively, whereas estimates for the TRC were 0.37 and 0.18 . Depending on estimated carrying capacity, the strength of the density dependence, level of uncertainty, and the treatment of unresolved signals, persistence probabilities for the TRC subpopulation ranged from 0.295 to 0.999 .

Estimates of $N$ for females in the TRB ranged from 140 to 163 during 2006-12 when detection heterogeneity was assumed to follow a logistic-normal distribution (Model 1) and from 133 to 158 when a 2-point finite mixture distribution was assumed (Model 2). Annual estimates of $\gamma$ ranged from 0.00 to 0.16 and from 0.00 to 0.22 , depending on the model, and estimates of $\phi$
\end{abstract}

\footnotetext{
${ }^{1}$ Department of Forestry, Wildlife and Fisheries, 274 Ellington Plant Sciences Building, University of Tennessee, Knoxville, TN 37996

${ }^{2}$ U.S. Geological Survey, Southern Appalachian Field Branch, Leetown Science Center Department of Forestry, Wildlife and Fisheries, 274 Ellington Plant Sciences Building, University of Tennessee, Knoxville, TN 37996
} 
ranged from 0.87 to 0.93 during that period. In the UARB, estimates of $N$ for females ranged from 25 to 44 during the study period, regardless of heterogeneity model. Estimated $\gamma$ ranged from 0.00 to 0.41 , and $\phi$ ranged from 0.88 to 0.90 during that period. Estimated $N$ for females in the LARB ranged from 78 to 97 from 2010 to 2012 based on Model 1 and from 68 to 84 based on Model 2. Estimates of $\gamma$ were 0.00 for 2010-11 regardless of heterogeneity model and ranged from 0.24 to 0.31 for $2011-12$, depending on the model assumptions. We estimated $\phi$ as 0.81 for $2010-11$, and from 0.84 to 0.85 for 2011-12, depending on model assumptions.

On the basis of vital rate estimates from Model 1 of the CMR analysis, probability of persistence over 100 years for the TRB subpopulation was $>0.999,0.975$, and 0.958 for processonly, 50-percent (\%) credible interval (CI), and 95\% CI projections, respectively. Similarly, the probability of persistence based on Model 2 was $>0.999$, 0.982, and 0.958. For the UARB, probabilities of persistence based on Model 1 were $>0.999,0.971$, and 0.958 for process-only, $50 \%$ CI, and 95\% CI projections, respectively, and 0.993, 0.929, and 0.849 for Model 2. Using the telemetry and reproductive data from the TRC, probabilities of persistence were greater than or equal to 0.95 only for projections based on the most optimistic set of assumptions. Assuming that the dynamics of the TRB, TRC, and UARB subpopulations were independent and using the most pessimistic population-specific persistence probabilities (that is, 0.958, 0.295, and 0.849, respectively), the probability of persistence for bears in the overall population system was 0.996 .

Genetic methods were used to estimate interchange and structure between subpopulations in Louisiana and in Minnesota (MINN); Mississippi (MISS); and the White River Basin (WRB), Arkansas. Results from the all-population and the WRB-TRB clustering analyses indicate at least five genetically distinct subpopulations. The genetic clustering and migrant analyses combined with capture data provided direct evidence that interchange had occurred from the WRB to the TRB and MISS, from the TRB to MISS, from the UARB to the TRC, and from the TRC to the TRB. Indirect evidence that interchange occurred from the UARB to the TRC and from the UARB to the TRB by way of the TRC was documented. No evidence was found of interchange from any of the subpopulations to the WRB, UARB, or LARB.

From April 2010 to April 2012, global positioning system (GPS) radio collars were placed on 8 female and 23 male bears ranging from 1 to 11 years of age to develop a step-selection function model to predict routes and rates of interchange. For both males and females, the probability of a step being selected increased as the distance to natural land cover and agriculture at the end of the step decreased and as distance from roads at the end of a step increased. Of 4,000 correlated random walks, the least potential interchange was between TRB and TRC and between UARB and LARB, but the relative potential for natural interchange between UARB and TRC was high. The step-selection model predicted that dispersals between the LARB and UARB subpopulations were infrequent but possible for males and nearly nonexistent for females. No evidence of natural female dispersal between subpopulations has been documented thus far, which is also consistent with model predictions. Both the genetic data and the step-selection results illustrated the value of the reintroduced TRC subpopulation in facilitating connectivity.

\section{Introduction}

Habitat fragmentation is a fundamental cause of population decline and increased risk of extinction for many wildlife and plant species worldwide. The subdivision of contiguous populations into small isolated subpopulations can have serious demographic and genetic consequences that increase the likelihood of local extirpations and the eventual collapse of entire 
systems. For example, small populations are subject to increased probabilities of extinction compared with larger populations simply because of stochastic demographic processes (MacArthur and Wilson, 1967; Shaffer, 1987; Lande, 1993). Also, small populations are more prone to chance fixation of deleterious alleles caused by stochastic processes, such as genetic drift, founder effects, and inbreeding depression (Mills, 2007). Furthermore, populations composed of spatially discrete subpopulations often depend on dispersal to facilitate demographic rescue or re-colonization and to maintain genetic variability essential to long-term persistence (Hanski, 1996, McCullough, 1996; Anderson and Danielson, 1997; Duke and others, 2001). Finally, close spatial proximity may result in non-independent fates of individual subpopulations, resulting in an increased extinction risk for the entire system. Therefore, understanding how fragmented systems function is critical to the conservation management of species of concern.

Ursus americanus (American black bear) is the most common species of the three North American ursids and once occurred throughout the continent from northern Canada into Mexico (Pelton, 2003). Since European settlement, the historic range of the black bear has been reduced by nearly 25-35 percent with most of that reduction occurring in the contiguous United States (Scheick and McCown, 2014). Large contiguous populations continue to persist in mountainous regions such as the Rocky and Appalachian Mountains, largely because these rugged topographies are less prone to human development and exploitation. In contrast, human development in the Southeastern Coastal Plain has reduced bear populations, which now exist in small vestigial patches of forests consisting of mixtures of bottomland hardwood swamps, pocosins, and Pinus spp. (pine) plantations (Wooding and others, 1994).

Conservation and management priorities for southeastern bear populations include the need to alleviate the negative demographic and genetic consequences associated with habitat loss and fragmentation (Hellgren and Vaughan, 1994). Because population growth in bear populations is most sensitive to changes in adult female survival, factors affecting this vital rate have significant consequences for the future viability of bear populations in this region (Beston, 2011). Therefore, recovery efforts for populations at risk, such as those of Ursus americanus luteolus (Louisiana black bear), have recognized the importance of management strategies that increase the quality and quantity of habitat and reduce human-caused mortality (U.S. Fish and Wildlife Service [USFWS], 1995).

The Louisiana black bear once ranged throughout Louisiana, southern Mississippi, and eastern Texas and occurred in the greatest numbers in the bottomland hardwoods of the Lower Mississippi Alluvial Valley (LMAV; St. Amant, 1959). By the 1950s, much of the bottomland hardwood forests had been converted to agriculture, and the statewide bear population was estimated to be 80-120 bears equally distributed between the Tensas River Basin and the coastal part of the Atchafalaya River Basin (St. Amant, 1959). In response to low population numbers, the Louisiana Wild Life and Fisheries Commission (now Louisiana Department of Wildlife and Fisheries [LDWF]) initiated a reintroduction program from 1964 to 1967 during which 161 bears were captured in Cook County, Minnesota, and released in Louisiana, 31 in the Tensas River Basin and 130 in the Upper Atchafalaya River Basin (UARB; Taylor, 1971).

Bottomland hardwood forests in the LMAV remain highly fragmented with $>80$ percent lost primarily to land clearing for agriculture by 1980 (U.S. Fish and Wildlife Service, 1995). As a consequence, the remaining bears in the region exist in isolated fragments of wooded habitat in the Tensas and Atchafalaya River Basins. In 1992, the USFWS classified the Louisiana black bear as Threatened under the U.S. Endangered Species Act (ESA) of 1973, listing loss and fragmentation of habitat as the primary threats (U.S. Fish and Wildlife Service, 1992). The 1995 Recovery Plan 
outlines recovery goals designed to meet the objective of reducing threats to the Louisiana black bear and the supporting habitat (U.S. Fish and Wildlife Service, 1995). To meet that objective, the Recovery Plan lists the following criteria for delisting:

1. at least 2 viable subpopulations, 1 each in the Tensas and Atchafalaya River Basins;

2. establishment of immigration and emigration corridors between the 2 viable subpopulations; and

3. long-term protection of the habitat and interconnecting corridors that support each of the 2 viable subpopulations used as justification for delisting.

The Recovery Plan defines a viable subpopulation as one that has a 95-percent or better chance of persistence over 100 years, despite random effects of demography, environment, genetics, and natural catastrophes. Long-term protection is defined as having sufficient voluntary conservation agreements with private landowners and public land managers so that habitat degradation is unlikely to occur over 100 years. Although the Recovery Plan was not explicit in defining how it would be determined whether or not the corridor provision was met, the document does describe the functional attributes of corridors, stating that "Corridors providing cover may facilitate the movement of bears between highly fragmented forest habitats (Pelton, 1982; Noss, 1987). If adequate immigration and emigration exists between habitat patches, small numbers of bears can function as a viable population (Lande, 1987).” Thus, the Recovery Plan implies that the identification and conservation of crucial habitat blocks and corridors may be required to facilitate the movement of bears among fragmented forest habitats. Note that the 1995 Recovery Plan classifies bears along the Louisiana coast in the Lower Atchafalaya River Basin (LARB; Iberia and St. Mary Parishes) and bears in the Upper Atchafalaya River Basin (Pointe Coupee Parish) as subpopulations, and the two together constitute the Atchafalaya River Basin population (fig. 1). 


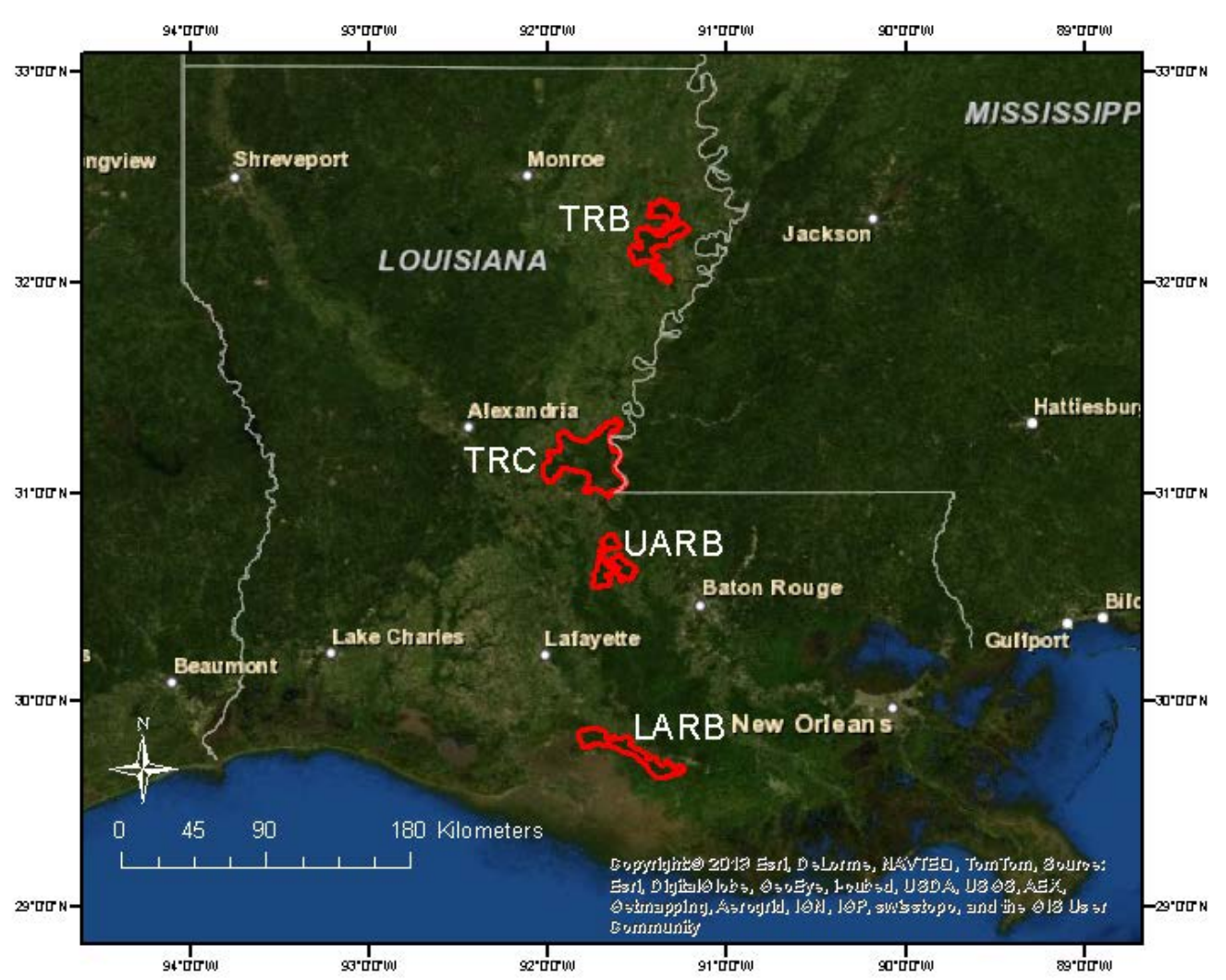

Figure 1. Study area showing each of the four subpopulations of Louisiana black bear (red polygons) within the Lower Mississippi Alluvial Valley in Louisiana. Subpopulations studied were located at Tensas River Basin (TRC), Three Rivers Complex (TRC), Upper Atchafalaya River Basin (UARB), and Lower Atchafalaya River Basin (LARB).

Many studies on Louisiana black bears have been conducted since the Recovery Plan was published and prior to the initiation of this research project. Research focused on movement patterns (Marchinton, 1995; Nyland, 1995; Anderson, 1997; Beausoleil, 1999; Wagner and others, 2001; Hightower 2003; Benson and Chamberlain, 2007), habitat needs (Weaver, 1990; Stinson, 1996; Bowman, 1999), taxonomy (Warrillow and others, 2001; Kennedy and others, 2002; Csiki and others, 2003; Triant and others, 2004), denning ecology (Weaver and Pelton, 1994; Hightower and others, 2002; Crook and Chamberlain, 2010), public attitudes (Bowman and others, 2001; Van Why and Chamberlain, 2003b), mortality (Pace and others, 2000; Van Why and Chamberlain, 2003a), and population abundance (Beausoleil, 1999; Boersen and others, 2003; Triant and others, 2004). Most recently, Hooker (2010), Lowe (2011), and Troxler (2013) estimated bear population sizes at the Tensas River Basin (TRB), Upper Atchafalaya River Basin (UARB), and Lower Atchafalaya River Basin (LARB), respectively, and O'Connell (2013) and O'Connell and others (2014) updated population estimates and evaluated the effects of the opening of the Morganza Spillway on bear demographics at UARB. 
Along with research, a number of management activities have improved recovery prospects for the Louisiana black bear. In 2009, the USFWS designated approximately 484,000 hectares (ha) of Federal, state, and privately owned lands as Critical Habitat for the Louisiana black bear under the ESA (U.S. Fish and Wildlife Service, 2009). Since 1992, a total of 22,263 ha of future bear habitat was created under the Federal Wetland Reserve Program, and a total of 3,654 ha was protected through the establishment of Bayou Teche National Wildlife Refuge, adding to the existing 115,500 ha of Federal and state lands within the boundaries of the Critical Habitat designation (U.S. Fish and Wildlife Service, 2009). Additionally, a reintroduction program was conducted from 2001 to 2009 to reestablish a subpopulation in the Three Rivers Complex (TRC) in east-central Louisiana between the TRB and UARB (fig. 1). The primary objective of this program was to translocate breeding-age females from the TRB to suitable but vacant habitat, thereby establishing another breeding subpopulation that would increase overall numbers and strengthen the network of bear subpopulations in the region. Since inception of the reintroduction program, 48 adult females with 104 cubs have been translocated to the TRC. Although the TRC subpopulation was not identified in the 1995 Recovery Plan, the intent of the reintroduction was for the TRC subpopulation to function as a stepping stone, increasing connectivity between the UARB and TRB, and to act as a numeric buffer, thus increasing the probability of persistence for the metapopulation.

Although there have been many positive developments, whether Louisiana black bears can persist in Louisiana for the long-term has not been established. The Recovery Plan generally refers to two subpopulations consisting of bears in the Tensas River Basin and those in the Atchafalaya River Basin. In 2014, researchers and managers generally consider there to be four distinct breeding subpopulations of Louisiana black bears, consisting of TRB, UARB, LARB, and the reintroduced TRC subpopulations (fig. 1), which we collectively refer to as the population system. Therefore, to estimate persistence of the Louisiana black bear, a unified evaluation of Louisiana black bear recovery throughout the entire Lower Mississippi Alluvial Valley (LMAV) of Louisiana is needed. This will first require an evaluation of the long-term viability of each of the subpopulations by forecasting individual subpopulation trajectories. Once the viability of each subpopulation is assessed, a comprehensive viability analysis for all subpopulations can be achieved by forecasting the trajectory of the entire Louisiana system. Furthermore, genetic and demographic interchange and pathways for such interchange within the network of populations within the LMAV of Louisiana is essential to long-term viability, and a better understanding of such interchange is needed. This information will then be combined to provide a holistic evaluation of Louisiana black bear recovery throughout the entire LMAV of Louisiana.

The objectives of the study were to

1. estimate demographic rates of Louisiana black bear subpopulations,

2. develop data-driven stochastic population projection models to assess long-term persistence of individual subpopulations and the overall black bear population in Louisiana,

3. determine how different assumptions with respect to projection model structure and parameter values affect population trajectories and long-term persistence, and

4. evaluate genetic structure and movement dynamics of Louisiana black bear subpopulations.

\section{Purpose and Scope}

This report describes population attributes and movements of the Louisiana black bear from 2002-2012 throughout the state, but primarily at four sites. Those sites are the Tensas River 
Basin (TRB) in northeast Louisiana, the Three Rivers Complex (TRC) in east-central Louisiana, the Upper Atchafalaya River Basin (UARB) in east-central Louisiana, and the Lower Atchafalaya River Basin (LARB) in south-central Louisiana along the Gulf of Mexico. This report addresses population viability and connectivity issues as pertaining to the 1995 Recovery Plan (U.S. Fish and Wildlife Service 1995).

\section{Description of Study Area}

The study area included the entire LMAV of Louisiana and western Mississippi, but the collection of field data was restricted to the three original subpopulations and the reintroduced subpopulation at TRC (fig. 1). Most of Louisiana is Outer Coastal Plain Mixed Forest (that is, uplands) and Lower Mississippi Riverine Forest (that is, alluvial; Bailey, 1995). The uplands consist of prairie and woodlands, whereas the alluvial region includes swamps, coastal marshes, beaches, and barrier islands. Elevations range from 0 at the coast to 163 meters (m) above NGVD 29 at Driskill Mountain in the uplands. The riverine system is extensive, consisting of $>6,400$ kilometers $(\mathrm{km})$ of navigable waterways.

The study area has a humid subtropical climate with long, hot, humid summers and short, mild winters. Average annual temperatures ranged from 16 to 21 degrees Celsius $\left({ }^{\circ} \mathrm{C}\right)$. Rainfall is abundant and well distributed throughout the year; annual precipitation at Baton Rouge averaged 153.9 centimeters (cm; http://www.usclimatedata.com/climate/baton-rouge/louisiana/unitedstates/usla0033). Historically, much of Louisiana was covered by bottomland deciduous forest with an abundance of Fraxinus spp. (ash), Ulmus spp. (elm), Populus deltoides (cottonwood), Celtis laevigata (sugarberry), Liquidambar styraciflua (sweetgum), Nyssa aquatica (water tupelo), Quercus spp. (oak), and Taxodium distichum (baldcypress). Upland areas consisted of Pinus taeda (loblolly) and Pinus echinata (shortleaf pine). Much of the alluvial area has since been converted to agriculture, primarily corn, soybeans, and wheat (Neal, 1990).

\section{Methods}

\section{Population Viability Analyses}

\section{Data Sources}

Data were collected from four primary research activities to assess population viability: (1) live capture, (2) winter den visits, (3) radio monitoring of individuals fitted with VHF transmitters, and (4) non-invasive DNA sampling. Additional data were opportunistically collected from sightings, road mortalities, and human-bear conflict management activities throughout the LMAV of Louisiana. Data collection was conducted by students, technicians, and biologists from the University of Tennessee, Louisiana State University, and LDWF during 2002-12 in the four areas supporting breeding populations. Viability analyses were done on two main datasets - the telemetry data from TRB and TRC, and the capture-mark-recapture (CMR) data from TRB, UARB, and LARB. 


\section{Live Capture and Radio Tagging}

Black bears were captured each year from 2002 to 2011 for several projects with various research and management objectives, including investigations of habitat use, denning ecology, reproduction, survival, movement patterns, and translocation. Bears were captured using modified Aldrich spring-activated foot snares (Aldrich Animal Trap Company, Clallam Bay, Washington) or culvert traps. Traps were checked once daily, except during extremely hot weather (that is, $>35^{\circ} \mathrm{C}$ ) when traps were checked twice daily or disabled during diurnal hours. Bears were immobilized using 4.4 milligrams (mg) of ketamine hydrochloride and $2.2 \mathrm{mg}$ of xylazine hydrochloride per kilogram (kg) or using 4-5 mg of Telazol ${ }^{\circledR}$ (Fort Dodge Animal Health, Fort Dodge, Iowa) per kg of estimated body mass. After latency, bears were placed in lateral or sternal recumbency, sterile ophthalmic lubricant was applied to prevent corneal desiccation, and blindfolds were secured to reduce visual stimulation and prevent retinal damage. Body temperature, respiration, and pulse were monitored throughout each immobilization. Yohimbine hydrochloride was intravenously administered at a dosage of $0.2 \mathrm{mg}$ per $\mathrm{kg}$ of estimated body mass as an antagonist for bears immobilized with ketamine and xylazine.

Female bears greater than or equal to $(\geq) 36.4 \mathrm{~kg}$ captured from 2002 to 2005 and females $\geq 45.5 \mathrm{~kg}$ captured from 2006 to 2011 were fitted with mortality sensitive radio collars (Advanced Telemetry Systems, Isanti, Minnesota; Telonics, Mesa, Arizona). All collars incorporated a leather spacer soaked in oil to serve as a release mechanism. Unmarked individuals received unique lip tattoos, plastic ear tags, and passive integrated transponder (PIT) tags. Existing marks, morphometric measurements, estimated age class, general condition, and reproductive status were recorded for all bears. The first upper premolars were extracted for age determination by cementum annuli analysis (Willey, 1974). Animals were handled according to University of Tennessee Institutional Animal Care and Use Committee (IACUC) protocol number 1716 and Louisiana State University IACUC protocol number A-03-04.

\section{Winter Den Visits}

From 2003 to 2013, radio-collared females were located by VHF signal during JanuaryMarch to determine reproductive status and litter size. When feasible, females wearing VHF collars nearing the end of battery life were immobilized to replace collars using the same immobilization drugs and procedures as live-captured bears. Cubs were weighed and sexed, and PIT tags were implanted. Hair samples were collected for DNA analysis. Additionally, selected females (that is, accessible and with cubs of the year) were immobilized for translocation to the TRC from 2001 to 2009 as part of the reintroduction program.

\section{Radio Monitoring}

Radio monitoring was conducted in the TRB and TRC at various intensities and durations according to different research objectives during the time span of this study. From 2003 to 2005, adult females were located using ground telemetry three or more times per week during the active months (April-November) to determine space use of resident bears in the TRB and of bears recently released into the TRC (Benson, 2005). Radio monitoring resumed in the TRC in 2006 and the TRB in 2007 and continued through 2012 with bi-monthly or monthly telemetry flights during non-denning months to monitor survival for adult females in the TRB and all bears reintroduced to the TRC. From 2002 to 2012, collared females were opportunistically radio located by ground 
telemetry to conduct post-den emergence observations of family groups in the TRB and TRC to verify reproductive status. Females were then approached on foot to determine reproductive state (barren [B], with cubs [C], or with yearlings [Y]) and to record observed litter size.

\section{Survival Rates of Radio-Collared Adult Female Bears}

Survival rate of independent females is a critical demographic component of black bear population dynamics (Beston, 2011). Determining how individual and environmental covariates affect survival rates and, hence, population growth is important in assessing population trends. Monthly encounter histories were constructed from radio monitoring data for the adult female survival analysis. April 1 of each year was used as the start date of the annual survival period to coincide with the period when females generally become active following den emergence. Each bear was considered initially available during the month it was radio collared and continued to be considered available until it died or was censored. Confirmed mortalities were assigned to month of known death or month of last known active signal. It was assumed that bears that shed their collars (that is, leather spacer broke and released collar or collar slipped off bear) were alive at the time of last active signal, and encounter histories of those bears were right censored to the month of the last active signal. Bears that were not encountered in more than 2 consecutive months but were subsequently re-encountered were right censored to the month of the last active signal and were re-entered into the dataset for the month of re-encounter. Because non-parturient females can be active during the winter den season in Louisiana, we did not assume survival was 1.0 during that period and applied the same censoring rule throughout the entire year.

Process variation of demographic rates refers to the manner in which those rates vary over time, space, and individuals. Estimates of process variance over time (hereafter referred to as temporal process variance) are important for incorporating temporal variation that is not explained by ancillary covariates into population projection models (White, 2000). Variance estimates obtained from time series of demographic rate estimates are not appropriate estimates of temporal process variation because of the added effects of sampling variation on variation in the time series. Therefore, separating temporal process and sampling variation was necessary.

Ideally, the most important factors influencing such variation are known, are measureable, and can be used to characterize or forecast variation in demographic rates and population dynamics. More often, such factors are unknown or operate in such complex ways that they cannot be identified or measured because sufficient data to do so are not available. Fortunately, the cumulative effects of such complex factors often can be characterized by general stochastic processes based on known families of probability distributions for which governing parameters can be estimated from available data and then used in population forecasts. When time series of demographic data were sufficient in length (that is, $\geq 6$ years), we estimated temporal process variation for survival and other demographic rates.

Numerous methods are available that can be used to separate the sources of variation. In general, a hierarchical modeling approach within a Bayesian estimation framework was used to separately estimate temporal process variation and sampling variation. To do so, a hyperdistribution structure was imposed on annual demographic rates, whereby annual rates were modeled as coming from a normal distribution governed by a mean that represented the expected value over time and a variance term that represented the magnitude of temporal process variation. The Bayesian estimation procedure produced a set of values that represented a sample from the posterior distribution and provided an estimate of parameter uncertainty caused by sampling 
variation. Spatial or individual process variation was not estimated because sufficient data to do so were not available.

From 2001 to 2009, radio-collared adult female bears with cubs were translocated from the TRB to the TRC during the winter den season to reestablish a breeding population. From the perspective of the TRB population, those females essentially were losses. However, treating those animals as losses can negatively bias estimates because only radio-collared individuals were exposed to translocation (Clark and Eastridge, 2006). Therefore, we right-censored translocated TRB females to the month of translocation and re-entered them into the dataset as new TRC females for the same month.

Occasionally, loss of radio signal caused by battery depletion, malfunction, or inaccessibility prevented collar recovery and fate determination. When fates of individuals were unknown, a maximum survival estimate treating missing animals as alive and a minimum survival estimate treating missing animals as dead can be obtained that provide an upper and lower bound for survival (Heisey and Fuller, 1985; Pollock and others, 1989; Haroldson and others, 2006). To bound survival estimates, two datasets were constructed either by assuming radio-collared bears with unknown fates were alive and right censoring those to the month of last active signal (that is, assumed censored, AC) or by assuming they died with mortality assigned to the month of last active signal (that is, assumed dead, AD). The latter scenario is relevant because poachers sometimes destroy radio collars after killing the animal and, if this occurs to any extent, assuming that signal loss is not related to mortality can produce positively biased estimates of survival. Both estimates were used in the population projections to provide pessimistic and optimistic estimates of growth.

A parametric exponential model of survival time with a constant discrete hazard rate function and a hierarchical modeling approach was used to estimate population-specific annual survival rates for female black bears in the TRB and TRC. To estimate mean annual survival rates, temporal process variances, and process correlation, annual survival rates for each population were treated as random effects by imposing a hierarchical model structure, whereby annual log hazard rates were modeled as random realizations from a common bivariate normal hyperdistribution. Annual survival $(S)$ was defined as

$$
S_{i, j}(t)=e^{-H_{i, j}(t)}
$$

where $H_{i, j}(t)=\sum_{t=1}^{12} h_{i, j}(t)$ is the cumulative discrete hazard and $h_{i, j}(t)=e^{\left(\delta_{i, j}\right)}$ is the unit (monthly) hazard rate. Subscripts $i$ and $j$ are indexed years and populations, respectively, and $\delta$ is defined as the baseline log hazard rate. The inverse Wishart distribution with two parameters, a scale matrix $(R)$ and degrees of freedom $(d f)$, was used as the prior for the variance-covariance matrix of the bivariate normal hyperdistribution. $R$ was specified as

$$
\left[\begin{array}{ll}
1 & 0 \\
0 & 1
\end{array}\right] \text { with } d f=3
$$

Uniform priors of Unif $(-15,0)$ were specified for the means of the bivariate normal hyperdistribution. Priors $>0$ resulted in extremely low annual survival rates (less than $[<] 6.1 \mathrm{E}-6$ ), so we truncated the prior to increase efficiency of the Markov chain Monte Carlo (MCMC) sampler. 


\section{Reproductive Rates of Radio-Collared Adult Female Bears}

To estimate reproductive rates in the TRB and TRC, reproductive status data collected during winter den visits were used. First, a multi-state transition modeling approach was used to estimate the probability that a female was in reproductive state B, state C, or state Y during winter, given her reproductive state during the previous winter (Schwartz and White, 2008). This approach assumes transitions between states are first-order Markovian processes and differs from the classical multi-state CMR modeling approach (Arnason, 1972) in that apparent survival and detection probabilities are assumed to be 1 . That assumption was made because only data on females that survived and for which reproductive status was observed in consecutive years were analyzed. We separately estimated transition probabilities for the TRB and TRC to compare rates between the two populations. Because transitions from B to Y and from Y to $\mathrm{Y}$ were not biologically possible, those transition probabilities were fixed at 0 . To ensure transition probabilities were restricted to the interval $[0,1]$ and met the unit-sum constraint requirement for transitions from one state to all other states, we indirectly imposed a Dirichlet prior for transition probabilities by specifying hyperpriors $\alpha_{i, j} \sim \operatorname{gamma}(1,1)$ and the relation

$$
\psi_{i, j}=\alpha_{i, j} / \sum_{j=1}^{n} \alpha_{i, j}
$$

where $\psi_{i, j}$ is the probability of transitioning from state $i$ to state $j$ (Royle and Dorazio, 2008; Kery and Schaub, 2012).

Assuming transition probabilities were constant across time and age classes, we next estimated the posterior distributions of reproductive state probabilities (that is, proportion of females in each reproductive state) by multiplying a state vector representing all possible reproductive states (for example, $[1,0,0]$ ) by the transition matrix from each MCMC sample and repeating the procedure 50 times using the resulting vector from the previous iteration. The distributions of stable state probabilities for females in different reproductive states for the TRB were compared to those for the TRC to identify potential differences in reproduction and litter survival processes. From the estimated stable reproductive state probabilities, the proportion of females with cubs or with yearlings can be multiplied by the mean litter size of those age classes to obtain an estimate of fecundity rate or yearling recruitment for breeding females, which can be used to project the number of cubs or yearlings in population projections. However, measures of fecundity and yearling recruitment based on mean litter size pose problems for population projections because they are discrete processes, whereas mean litter size is on a continuous scale. Therefore, we chose to independently model litter size probabilities for cub and yearling age classes as a multinomial process, whereby each possible litter size was treated as a categorical response variable on the nominal scale.

Observed litter size data for females with cubs collected during winter den visits in the TRB and TRC and multinomial logistic regression were used to estimate the probability of a female producing a 1-, 2-, 3-, or 4-cub litter, conditional that the female was in the $\mathrm{C}$ reproductive state. Similarly, observed litter size data for females with yearlings were used to estimate litter size probabilities for that age class. Litter size probabilities for the TRB and TRC were separately estimated and a Dirichlet prior via gamma hyperpriors was used to ensure probabilities were restricted to the interval $[0,1]$ and met the unit-sum constraint requirement. 
An estimate was derived for mean litter size by first calculating the posterior distribution for mean litter size as

$$
\bar{L}_{i}=\sum_{j=1}^{4} \operatorname{Pr}\left(L_{i, j}\right) \times L_{i, j},
$$

where $\operatorname{Pr}\left(L_{i, j}\right)$ is the probability of litter size $j$ and $L_{i, j}$ is litter size $j$ for the ith sample and then calculating the mode of that distribution. To derive an estimate of fecundity ( $f$; the number of cubs produced per breeding age female) and yearling recruitment ( $r$; number of yearlings produced per breeding age female) for each study area, the posterior distribution was obtained for each by calculating the pairwise product of 1,000,000 random values drawn from the posterior distribution of mean cub or yearling litter size and 1,000,000 random values drawn from the posterior distribution of the corresponding $\mathrm{C}$ or $\mathrm{Y}$ stable state probability and then multiplying each product by 0.5 on the basis of an assumed 1:1 sex ratio for cubs and yearlings. Finally, $f$ and $r$ were estimated by calculating the modes of those posterior distributions.

Non-Invasive DNA Sampling

Non-invasive DNA sampling is based on the use of molecular markers to obtain unique, multilocus genotypes of individual animals. DNA was extracted from hair collected at baited, barbed-wire enclosures to determine individual identities, record capture histories for capturemark-recapture (CMR) analysis, ascertain population of origin, infer population structure, and study family relationships (Woods and others, 1999). Hair sampling was conducted for three subpopulations: TRB, UARB, and LARB. To ensure that all bears would have opportunities to be sampled, hair-collection sites were spaced so that four or more sites would be available per adult female home range (Otis and others, 1978). Site density, number of sites, and sampling area varied among study areas, depending on home range size, area of forested habitat, and accessibility (table 1).

Table 1. Distribution of hair-collection sites for study areas in the Tensas River Basin (TRB), Upper Atchafalaya River Basin (UARB), and Lower Atchafalaya River Basin (LARB), Louisiana.

$\left[\mathrm{km}^{2}\right.$, square kilometers]

\begin{tabular}{l|c|c|c|c|c}
\hline \multicolumn{1}{c|}{ Study area } & Home range size & Site density & $\begin{array}{c}\text { Sites per home } \\
\text { range }\end{array}$ & Number of sites & Sampling area sized \\
\hline TRB & $10.0 \mathrm{~km}^{2}$ & $1 / 3.8 \mathrm{~km}^{2}$ & 2.63 & 209 & $795 \mathrm{~km}^{2}$ \\
UARB & $15.7 \mathrm{~km}^{2}$ & $1 / 5.0 \mathrm{~km}^{2}$ & 3.14 & 115 & $575 \mathrm{~km}^{2}$ \\
LARB & $11.8 \mathrm{~km}^{2}$ & $1 / 5.2 \mathrm{~km}^{2}$ & 2.27 & 118 & $613 \mathrm{~km}^{2}$ \\
\hline
\end{tabular}

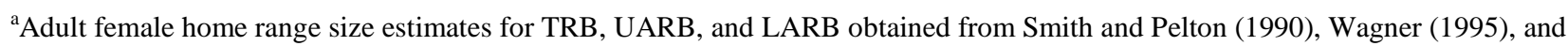
Murrow and Clark (2012), respectively.

${ }^{\mathrm{b}}$ Site density = Number of sites/area size.

${ }^{\mathrm{c}}$ Sites per home range $=$ home range $\times$ site density.

${ }^{\mathrm{d}}$ Sampling area was estimated by circumscribing each site by a circle with a radius equal to that of an adult female home range, merging those circles into a single polygon, and calculating the area contained within that polygon. Non-forested habitat was not excluded from these estimates.

From 2006 to 2009, hair-collection sites were established consisting of a single strand of 4point, 15.5-gauge barbed wire $40-50 \mathrm{~cm}$ above the ground, stretched around $3-5$ trees and enclosing an area approximately $5 \mathrm{~m} \times 5 \mathrm{~m}$. Beginning in 2010, sites were constructed using two strands of barbed wire at $35-40 \mathrm{~cm}$ and $65-70 \mathrm{~cm}$ above the ground to increase the likelihood of 
collecting hair from bears that avoided detection by crawling under or stepping over the single wire. Each site was baited with a small amount of bakery products (for example, sweet rolls, donuts) and a scent attractant (artificial raspberry or honey flavoring; Mother Murphy's Laboratories, Greensboro, North Carolina). All sites were checked for hair samples, and each site was rebaited every 7 days for 8 weeks each year. Hair was collected using this protocol for 7 years in the TRB (2006-12), 6 years in the UARB (2007-12), and 3 years in the LARB (2010-12). In 2012, we sampled for only 3 weeks in the TRB because research objectives changed to less intensive long-term monitoring of population trends (Maria Davidson, LDWF, oral commun., 2012). Samples collected from individual barbs were each placed in individually labeled coin envelopes and stored in a dry location at room temperature until DNA extraction was performed. To ensure sufficient DNA for sequence analysis, only samples with at least five hairs were collected. To prevent contamination with future hair samples, a cigarette lighter or propane torch was used to burn any remaining hair from the barbs after sample collection.

Small home ranges and high population densities often require greater densities of haircollection sites to ensure all bears have a non-zero probability of being captured. Such site densities can produce a large number of hair samples (Settlage and others, 2008). Because genotyping all samples was cost prohibitive, we selected only a portion of the total number of samples collected (that is, subsample) for DNA analysis. The number of subsamples and the method of selection sometimes varied by study area to ensure optimal spatial coverage and to achieve adequate capture probabilities for CMR analyses.

In the TRB, the subsampling objective was to submit 75 viable samples (that is, samples containing five or more guard hairs or combination of guard hairs and underfur hairs) per week for DNA analysis. That number was based on a combination of budget constraints and the need to obtain adequate capture probabilities (Laufenberg, 2014). We accomplished this by selecting 1 viable sample from 75 randomly selected sites each week from those sites that produced 1 or more samples. For each selected site, samples were examined in random order to select the first viable sample. If no viable samples were available for a given site, the site was passed over. If the number of unique sites that produced one or more viable samples in a given week was $<75$, we randomly reselected sites in search of additional viable samples to reach the target of 75 samples. In the UARB, the subsampling objective was 38 samples per week, and samples were selected using the same subsampling approach as for the TRB. In contrast, subsampling in the LARB was conducted by searching all site/week combinations for a viable sample in random order until 533 samples were selected each year. Similar to the TRB and UARB, if the number of unique site/week combinations that produced one or more viable samples was $<533$, site/week combinations were randomly reselected to find additional samples to reach the target.

We also submitted samples collected from individuals handled from live captures, den checks, and road mortalities in all four primary study areas for DNA analysis to supplement the non-invasive dataset. Small sections of foot pad tissue from road-killed bears, approximately 0.25 square centimeters $\left(\mathrm{cm}^{2}\right)$ in size, were placed in individually labeled coin envelopes and stored in a dry location at room temperature until DNA extraction was performed.

DNA extraction and microsatellite genotyping took place at Wildlife Genetics International, Inc. (Nelson, BC, Canada) following standard protocols (Woods and others, 1999; Paetkau, 2003; Roon and others, 2005). DNA was extracted from selected hair or tissue samples using QIAGEN's DNeasy Tissue kits. Guard hair roots were clipped and used for extraction, whereas in the case of underfur, entire clumps were used for extraction. The quantity of guard hairs and underfur used for extraction was recorded for each sample. 
Extracted DNA was amplified at all loci using polymerase chain reaction. Reactions contained 50 nanometers of potassium chloride, 0.1 percent Triton X-100, and 160 micrometers of deoxyribonucleotide triphosphates in a volume of 15 microliters with concentrations of magnesium chloride $\left(\mathrm{MgCl}_{2}\right)$, Taq polymerase and with primers optimized to permit co-amplification. Thermal cycling was performed using a Perkin Elmer 9600 instrument (Perkin Elmer, Waltham, Massachusetts). Amplified DNA samples were sequenced on a 373A automated sequencer (Applied Biosystems [ABI], Foster City, California) using ABI's four-color detection system. DNA fragments were analyzed, and genotype data were generated using Genescan software (ABI). Genotypes were determined using Genotyper software (ABI). Genotyping followed a three-phase approach to assign individual identities to samples submitted each year and to minimize genotyping errors that could cause misidentification of individuals (Paetkau, 2003).

The power of multilocus genotypes to differentiate individuals depends on the number and variability of markers used for individual identification and the number of individuals sampled (Paetkau, 2003). Marker variability was expected to be low and to differ among study areas because the bear population in Louisiana was substantially fragmented and reduced in size between 1890 and 1950 (St. Amant, 1959). Additionally, LDWF released 130 and 31 bears from Minnesota into the TRB and UARB, respectively, from 1964 to 1967 (Taylor, 1971), which affected genetic variation in those areas. For those reasons, genetic marker systems for individual identification were independently developed for each study area to ensure adequate power. Marker selection began by genotyping about 30 individuals from each population at 23 microsatellite markers to provide information on expected heterozygosity $\left(H_{\mathrm{E}}\right)$ that could be used to identify a smaller, optimal set of markers for each population. Paetkau (2003) suggested that small projects (that is, $<100$ sampled individuals) require $H_{\mathrm{E}} \geq 0.69$ for 6-marker systems to reliably distinguish between individuals, whereas $H_{\mathrm{E}} \geq 0.75$ is needed for larger projects (200-400 sampled individuals) using the same number of markers. In all study areas, variability based on the initial 23-marker analysis was lower than recommended for a 6-marker system, confirming the need for additional markers. On the basis of individual $H_{\mathrm{E}}$ of the 23 markers available for black bears and the efficiency of various markers to be simultaneously analyzed (that is, multi-plexing), subsets of markers with the greatest power to differentiate individuals were identified and selected for individual identification in each study area (table 2). Additionally, a region of the amelogenin gene was sequenced for all submitted samples to determine sex (Ennis and Gallagher, 1994) and was used to supplement microsatellite markers in resolving individual identities. 
Table 2. Microsatellite markers used for individual identification and population genetics analyses for Louisiana black bears in the Tensas River Basin (TRB), Upper Atchafalaya River Basin (UARB), and Lower Atchafalaya River Basin (LARB), Louisiana, 2006-12.

\begin{tabular}{|c|c|c|c|c|c|}
\hline Marker name & $\begin{array}{c}\text { GENBANK accession } \\
\text { code } \\
\end{array}$ & TRB & UARB & LARB & $\begin{array}{c}\text { Population } \\
\text { genetics }\end{array}$ \\
\hline $\mathrm{CPH9}^{\mathrm{a}}$ & GU179031.1 & & & & $\mathrm{X}$ \\
\hline CXX110 & N/A & & & $\mathrm{X}$ & $\mathrm{X}$ \\
\hline CXX20 & N/A & & & & $\mathrm{X}$ \\
\hline $\mathrm{G} 0 \mathrm{~B}^{\mathrm{c}}$ & U22084.1 & $\mathrm{X}$ & & & $\mathrm{X}$ \\
\hline G10C ${ }^{d}$ & U22085.1 & & $\mathrm{X}$ & & $\mathrm{X}$ \\
\hline $\mathrm{G} 10 \mathrm{H}^{\mathrm{c}}$ & U22086.1 & & & & $\mathrm{X}$ \\
\hline $\mathrm{G} 10 \mathrm{~J}^{\mathrm{c}}$ & U22087.1 & & & $\mathrm{X}$ & $\mathrm{X}$ \\
\hline $\mathrm{G} 10 \mathrm{~L}^{\mathrm{c}}$ & U22088.1 & & $\mathrm{X}$ & & $\mathrm{X}$ \\
\hline $\mathrm{G}_{10 \mathrm{M}^{\mathrm{d}}}$ & U22089.1 & $\mathrm{X}$ & $\mathrm{X}$ & & $\mathrm{X}$ \\
\hline $\mathrm{G} 10 \mathrm{P}^{\mathrm{d}}$ & U22091.1 & $\mathrm{X}$ & $\mathrm{X}$ & $\mathrm{X}$ & $\mathrm{X}$ \\
\hline $\mathrm{G} 10 \mathrm{U}^{\mathrm{c}}$ & U22092.1 & & & $\mathrm{X}$ & $\mathrm{X}$ \\
\hline G10X ${ }^{d}$ & U22093.1 & & & & $\mathrm{X}$ \\
\hline $\mathrm{G} 1 \mathrm{~A}^{\mathrm{c}}$ & U22095.1 & & & & $\mathrm{X}$ \\
\hline $\mathrm{G}^{\mathrm{c}} \mathrm{D}^{\mathrm{C}}$ & U22094.1 & $\mathrm{X}$ & & & $\mathrm{X}$ \\
\hline MSUT- $2^{\mathrm{e}}$ & AB040107.1 & $\mathrm{X}$ & & & $\mathrm{X}$ \\
\hline REN144A06 ${ }^{f}$ & AJ411278 & & & & $\mathrm{X}$ \\
\hline REN145P07 ${ }^{\mathrm{f}}$ & AJ411284 & & & & $\mathrm{X}$ \\
\hline UamD123 & EU414329 & & & $\mathrm{X}$ & $\mathrm{X}$ \\
\hline UamD1a ${ }^{g}$ & EU414318 & & & & $\mathrm{X}$ \\
\hline UarMU23 ${ }^{\mathrm{h}}$ & Y09645.1 & $\mathrm{X}$ & $\mathrm{X}$ & $\mathrm{X}$ & $\mathrm{X}$ \\
\hline UarMU26 ${ }^{\mathrm{h}}$ & Y09646.1 & & $\mathrm{X}$ & & $\mathrm{X}$ \\
\hline UarMU50 ${ }^{\mathrm{h}}$ & Y09647.1 & $\mathrm{X}$ & & $\mathrm{X}$ & $\mathrm{X}$ \\
\hline UarMU59 ${ }^{\mathrm{h}}$ & Y09649.1 & & $\mathrm{X}$ & $\mathrm{X}$ & $\mathrm{X}$ \\
\hline
\end{tabular}

${ }^{\mathrm{a}}$ Fredholm and Winterø, 1995.

broctor and others, 2002.

${ }^{\mathrm{C}}$ Paetkau and Strobeck, 1994.

${ }^{\mathrm{d}}$ Paetkau and others, 1995.

${ }^{\mathrm{e}}$ Kitahara and others, 2000.

${ }^{\mathrm{f}}$ Breen and others, 2001.

${ }^{\mathrm{g}}$ Meredith and others, 2009.

${ }^{\mathrm{h}}$ Taberlet and others, 1997.

To assess the power of the marker systems used to differentiate individuals, the probability that two full siblings randomly drawn from a population will have the same multilocus genotype ( $P I_{\text {sibs }}$, Taberlet and Luikart, 1999) was estimated. The $P I_{\text {sibs }}$ estimator represents a conservative upper limit of the probability of observing identical genotypes among individuals within a population (Taberlet and Luikart, 1999; Waits and others, 2001). Assuming random sampling of individuals, independence of alleles within loci, and no shared ancestry among individuals, $P I_{s i b s}$ at each locus is calculated as

$$
P I_{\text {sibs }}=0.25+\left(0.5 \sum p_{i}^{2}\right)+\left[0.5\left(\sum p_{i}^{2}\right)^{2}\right]-\left(0.25 \sum p_{i}^{4}\right),
$$

where $p_{i}$ is the frequency of the ith allele. Assuming independence of alleles among loci, an estimate of the multilocus $P I_{\text {sibs }}$ is obtained by taking the product of all loci-specific $P I_{\text {sibs }}$. To determine whether independence of loci and random sampling assumptions were met, we tested for linkage disequilibrium (lack of allele independence between loci) and conformity to HardyWeinberg equilibrium (independence of alleles within loci) in Program GENEPOP version 3.4 (Raymond and Rousset, 1995). The Dunn-Sidak method (Sokal and Rohlf, 1995) was used to 
ensure an experiment-wise error rate of $\alpha=0.05$ by restricting critical values for individual comparisons to $\alpha=1-(1-0.05)^{1 / k}$, where $k$ is the number of individual comparisons.

For each marker set, the frequency at which two individuals would match at all genotyped markers, including the sex marker (that is, zero-mismatch pairs or 0MM-pairs), was estimated for TRB, UARB, and LARB. To do so, genotype data from all individuals in each population were used to tally the number of pairs of individuals that mismatched at 1 to $k$ loci, where $k$ is the number of loci used in a marker set. The distribution of those numbers was plotted on the $\log _{10}$ scale against the number of mismatches, the slope of that distribution to 0MM-pairs was extrapolated, and an empirical estimate of the number of expected 0MM-pairs was visually derived (Paetkau, 2003). Genotyping of individuals handled in the TRC used the TRB marker subset because individuals handled in the TRC were translocated or descendants of those that were translocated from the TRB during the reintroduction project and would be genetically most similar.

Model Fitting, Estimation, and Inference

For all demographic rate analyses, MCMC sampling methods were used within a Bayesian inference framework implemented in JAGS (https://sourceforge.net/projects/mcmc-jags) accessed through Program R (Version 3.0.2, http://cran.us.r-project.org/, accessed January 30, 2014) with the package rjags (Plummer, 2011). Three independent MCMC sampling chains of 100,000 steps were collected after burn-in samples were discarded. Individual chains were inspected for serial correlation using autocorrelation function plots and were thinned to reduce within-chain serial correlation. Convergence was assessed by visually inspecting trace plots of the thinned chains and calculating the Gelman-Rubin diagnostic statistic using the gelman.diag function in the coda package for R (Plummer and others, 2006). Posterior modes for all parameter point estimates are reported unless specified otherwise. All analyses were conducted using vague, non-informative prior distributions.

\section{Demographic Rates from the Capture-Mark-Recapture Dataset}

The complete CMR dataset consisted of DNA-based binary detection records (that is, 1 if detected and 0 if not) of individual bears obtained from hair-collection surveys conducted across spatial arrays of hair-collection sites in the TRB, UARB, and LARB populations. Surveys were conducted in a robust-design format consisting of primary sampling occasions (that is, years), between which the population was considered open to gains and losses, and secondary occasions (that is, weeks) within primary occasions during which the population was considered geographically and demographically closed (Pollock, 1982). Selection of more than one sample for DNA analysis from individual site/week combinations occasionally caused the same bear to be detected more than once at a site during the same week. Additionally, individuals were often detected at one or more sites within a given week. Those multiple within-week detections were consolidated into single binary detection records. The final CMR dataset used for analysis consisted of binary records $\left(y_{i, k, t}\right)$ indicating whether individual $i(i=1, \ldots, n)$ was detected during week $k(k=1, \ldots, K)$ of year $t(t=1, \ldots, T)$, where $n$ is the total number of individuals ever detected, $K$ is the number of detection occasions within each year, and $T$ is the number of years.

The general approach to data analysis was to use a hierarchical CMR modeling framework based on a state-space parameterization of the Jolly-Seber model (Royle and Dorazio, 2008; Link and Barker, 2010) to estimate abundance $(N)$, annual apparent survival $(\phi)$, annual per capita 
recruitment $(\gamma)$, annual realized population rate-of-change $(\lambda)$, and weekly detection probabilities $(p)$ for females in the TRB, UARB, and LARB. Note that per capita recruitment is the ratio of the number of new recruits (that is, in situ reproduction or immigrants) to the total number of current residents (that is, breeding or non-breeding age) in the population and is different from fecundity and yearling recruitment (that is, $f$ and $r$ ) estimated from the telemetry data. The analysis was restricted to females because vital rates of females are more important determinants of population growth than those of males (Beston, 2011), because capture probabilities were greater, and because female reproductive rates are simpler to estimate. We considered $\phi$ and $\gamma$ for the TRB and UARB as random quantities using a hierarchical modeling approach to directly estimate temporal process variation $\sigma_{\phi}^{2}$ and $\sigma_{\gamma}^{2}$, respectively, while accounting for imperfect detection and sampling variation (Link and Barker, 2005). No attempt was made to estimate temporal variance in vital rates for the LARB because the number of years of data collection was insufficient for reliable estimation. Additionally, the relation between $\gamma$ and $N$ was modeled to test for density dependence in that vital rate because the failure to incorporate density effects into population projections when they are present in the population can lead to biased estimates of viability (Morris and Doak, 2002). A parameter-expanded data augmentation methodology was used to avoid technical problems caused by changes in the parameter space with each draw of the MCMC estimation procedure (Royle and others, 2007; Royle and Dorazio, 2012). This approach artificially inflates the number of individuals in the observed dataset with a fixed, known number of all-zero detection histories and includes an estimable zero-inflation parameter that represents the probability of inclusion in the population at the beginning of the study (Royle and others, 2007).

The basic structure of the state-space model formulation included 2 model components that describe the 3 ecological state processes of interest (that is, abundance, survival, and recruitment) and 1 model component that describes the observation state process (that is, detection) as follows: (1) a model for initial abundance during the first study year in each population, (2) a model for the change in abundance over time as a function of survival and recruitment, and (3) a model for the observation (that is, CMR) data. We first defined a latent state variable matrix $z$ of dimension $M \times T$, where element $z_{i, t}$ indicates whether individual $i$ is alive and has not permanently emigrated from the study area $\left(z_{i, t}=1\right)$ or is dead or has permanently emigrated $\left(z_{i, t}=0\right)$ at time $t$, $M$ is the sum of the total number of detected individuals across all study years $(n)$ and the number of all-zero detection histories used to augment the dataset, and $T$ is the number of study years. A number of individuals were selected with which to augment the observation data for each population that would result in $M$ being much greater than $n$ and avoid upper truncation of the posterior distribution for $N$.

The initial state of each individual in the augmented dataset was modeled as

$$
Z_{i, 1} \sim \operatorname{Bernoulli}(\psi)
$$

where $z_{i, 1}$ indicates whether individual $i$ is alive and a member of the sampled population at the beginning of the study and $\psi=\mathrm{E}\left(N_{1}\right) / M$ is the inclusion probability (Royle and Dorazio, 2008). As a result, initial abundance $\left(N_{1}\right)$ was defined as

$$
N_{1}=\sum_{i=1}^{M} Z_{i, 1}
$$

The second component of the ecological state process modeled abundance in years $t=2, \ldots, T$ as 


$$
z_{i, t} \sim \operatorname{Bernoulli}\left(z_{i, t-1} \times \phi+\left(1-\alpha_{i, t-1}\right) \times b\right),
$$

where $\alpha_{i, t-1}=\operatorname{maximum}\left(z_{i, t, \ldots,} z_{i, t-1}\right)$ indicates whether individual $i$ has already been recruited to the population and $b$ is recruitment probability. The state process equation defines the probability that an individual is alive and a member of the sampled population at time $t$ as $\phi$, given it was alive and in the study area at time $t-1$, and as $b$ if the individual had not previously been a member of the sampled population. We note that $\phi=S F$ where $S$ is the true annual probability of survival and $F$ is annual probability of fidelity to the study area because deaths and permanent emigration cannot be distinguished without ancillary information. The parameter $b$ is considered the probability of being recruited into the population. However, that probability is influenced by $M$ and has no direct biological interpretation. Per capita recruitment $(\gamma)$ is related to $b$ and is a more intuitive vital rate, which was defined as

$$
\gamma_{t}=\frac{b_{t} V_{t-1}}{N_{t-1}}, t=2, \ldots, T
$$

where $V_{t-1}=M-\sum_{i} a_{i, t-1}$ is the number of available recruits. We described the model component for the detection data as

$$
y_{i, j, t} \sim \operatorname{Bernoulli}\left(z_{i, t} \times p_{i, j, t}\right),
$$

where $p_{i, j, t}$ is the detection probability for individual $i$ during week $j$ of year $t$.

To separate sampling variance from process variance for $\phi$ and $\gamma$, annual values for each of those vital rates were treated as random variables coming from a common hyperdistribution using an appropriate link function. The hyperdistribution for $\phi$ was described as

$$
\begin{gathered}
\operatorname{logit}\left(\phi_{t}\right)=\mu_{\phi}+\varepsilon_{t} \\
\varepsilon_{t} \sim \operatorname{Normal}\left(0, \sigma_{\phi}^{2}\right),
\end{gathered}
$$

where $\mu_{\phi}$ is the overall mean annual apparent survival on the logit scale, $\varepsilon_{t}$ is the annual deviation from the mean, and $\sigma_{\phi}^{2}$ is the temporal variance. Similarly, temporal variation of $\gamma$ was modeled as

$$
\begin{gathered}
\log \left(\gamma_{t}\right)=\mu_{r}+\varepsilon_{t} \\
\varepsilon_{t} \sim \operatorname{Normal}\left(0, \sigma_{\gamma}{ }^{2}\right),
\end{gathered}
$$

where $\mu_{r}$ is the overall mean annual recruitment on the log scale, $\varepsilon_{t}$ is the annual deviation from the mean, and $\sigma_{\gamma}^{2}$ is the temporal variance.

Individual heterogeneity in $p$ is a well-known and prevalent issue when estimating vital rates for black bears from DNA-based CMR data (Tredick and others, 2007; Clark and others, 2010; Laufenberg and others, 2013). However, the most appropriate family of distributions (for example, beta, log-normal, or finite mixture) used to model individual heterogeneity is not identifiable using data-based selection criteria because different families can produce nearly identical data distributions but are parameterized by different values of $N$ (Link, 2003). An alternative approach to selecting a single distribution family is to consider multiple families and 
base inference on the entire set of models. Therefore, two common families of distributions were considered, the logistic-normal distribution (Coull and Agresti, 1999; Dorazio and Royle, 2003) and the finite-mixture distribution (Pledger, 2000). For the logistic-normal distribution (Model 1), $p$ was defined for individual $i$ during week $j$ in year $t$ as

$$
\begin{aligned}
& \operatorname{logit}\left(p_{i, j, t}\right)=\mu_{j, t}+\varepsilon_{i} \\
& \varepsilon_{i} \sim \operatorname{Normal}\left(0, \sigma^{2}\right),
\end{aligned}
$$

where $\mu_{j, t}$ is the mean weekly detection probability in year $t$ on the logit scale, $\varepsilon_{i}$ is the individual deviation from the mean, and $\sigma^{2}$ is the variance among individuals. For the finite-mixture distribution (Model 2), $p$ was defined for individual $i$ during week $j$ in year $t$ as

$$
\begin{gathered}
p_{i, j, t}=p_{i, t, g} \\
g \sim \text { Categorical }(A, \pi),
\end{gathered}
$$

where $p_{j, t, g}$ is the detection probability for mixture $g$ during week $j$ of year $t, A$ is the number of mixtures, and $\pi$ is a vector of probabilities defining the probability of an individual belonging to mixture $g$. For the analysis, $A$ was restricted to two mixtures. Detection probabilities likely differed across years in response to annual variation in abundance, distribution of food resources, weather, or other unknown factors. Therefore, $\mu_{j, t}$ and $p_{i, t, g}$ for the logit-normal and finite-mixture distributions were modeled as constant across weeks but with fixed-effect differences among years. In 2010, the hair-collection site configuration was modified from a 1-wire system to a 2-wire system that likely affected the distribution of individual differences in $p$. To account for this change in sampling methodology, we modeled with fixed-effects differences in $\sigma^{2}$ and $\pi$ for the logit-normal and finite-mixture distributions as 2 levels: pre- and post-modification. No temporal variation or behavioral effects in detection probabilities across weeks within years was assumed.

To model density dependence in per capita recruitment (Lebreton and Gimenez, 2013), we defined a log-linear model for the relation between $\gamma$ and $N$ as

$$
\begin{gathered}
\log \left(\gamma_{t}\right)=\beta_{0}+\beta_{1} N_{1}+\varepsilon_{t} \\
\varepsilon_{i} \sim \operatorname{Normal}\left(0, \sigma_{\gamma}{ }^{2}\right),
\end{gathered}
$$

where $\beta_{0}$ and $\beta_{1}$ are the intercept and slope parameters, respectively, $\varepsilon_{t}$ is the annual deviation from the mean, and $\sigma_{\gamma}^{2}$ is the temporal process variance.

The state-space formulation of the Jolly-Seber model often has extensive computational requirements because the latent state variable $z$ for each individual in each year must be updated at each step of the MCMC sampling process. For example, missing observations from the detection data between successive observations (for example, 1001 annual detection history) must be estimated because they are related to $z$ only through the observation process. This can result in extremely long periods of time required to achieve convergence and adequate mixing of multiple chains. One method to improve efficiency and reduce computation time is to directly impute information about $z$ for all years between the first and last year of observation and directly enter that information into the analysis (Kéry and Schaub, 2012). This was accomplished by creating a data matrix of known latent states where a 1 was recorded for all years an individual was known to 
be alive (for example, 1001 becomes 111 1), and NAs were recorded for years for which no information was available (for example, 1100 becomes 11 NA NA).

A rapidly developing approach for analyzing different types of population data in a single unified framework is integrated population modeling (Besbeas and others, 2002; Brooks and others, 2004; Schaub and others, 2007). This approach combines information collected from different sampling methods into a single population model facilitating simultaneous estimation of multiple vital rates and population processes that could not have been achieved if datasets were separately analyzed. Furthermore, use of integrated population models increases accuracy and precision when different types of data collected on the same vital rate (for example, CMR and known-fate data) are concurrently analyzed. Because hair samples collected from most of the females in the TRB known-fate dataset were genotyped, those genotypes could be matched to genotypes in the CMR dataset. This allowed direct incorporation of known-alive status information from the known-fate dataset into the known latent state matrix for the TRB analysis. Moreover, we incorporated information for bears in the CMR data from the TRB, UARB, and LARB that matched genotyped samples collected from bears handled during live-capture efforts for radio collaring, den-season captures for reproduction assessment, and conflict management activities.

In addition to including ancillary information about known-alive status, the known latent state matrix can also incorporate information from known mortalities. Therefore, known mortality data collected from radio monitoring and road mortality recoveries were used to incorporate known times of death into the analysis. During the first 4 years of CMR data collection in the TRB, females were being removed and translocated as part of the reintroduction efforts in the TRC. To account for removal of those females, zeroes were entered into the known latent state matrix. The following vague priors were used in all CMR models: $\mu_{\phi} \sim \operatorname{Normal}(0,0.001)$, $\beta_{0} \sim \operatorname{Normal}(0,0.0001), \beta_{1} \sim \operatorname{Normal}(0,0.0001), \sigma_{\phi}{ }^{2} \sim \operatorname{Uniform}(0,10), \sigma_{\gamma}{ }^{2} \sim$ Uniform( 0,10$)$, and $\psi \sim \operatorname{Beta}(1 \mathrm{E}-6,1)$. The median of the posterior distributions was used as a point estimator for $\sigma_{\phi}^{2}$ and $\sigma_{\gamma}^{2}$ because it is generally more robust when the level of variation is moderate and estimation is based on a time series of less than 7 years (White and others, 2009).

Determination of the demographic segment of the population that is being sampled is important in CMR-based studies of population dynamics because demographic rate estimates and inferences of population dynamics drawn from those estimates pertain only to the sampled population and may not reflect population segments that are not sampled. For DNA-based haircollection studies, young bears may never be detected because they are too small to encounter the barbed wire. To determine whether young bears were part of the sampled population, a search was performed of live-capture records for bears that were present in the TRB study area as cubs or yearlings during years of hair sampling and that had DNA samples collected during capture that were successfully genotyped. The DNA-based CMR dataset was searched for genotype matches, and the age at which each bear was first detected at a hair-collection site was determined. The numbers of bears detected at hair-collection sites as cubs, as yearlings, and as 2-year olds were tallied for use as measures of whether those age classes were part of the sampled population.

\section{Stochastic Population Modeling and Extinction Risk Assessment}

Population projections differ from population predictions. A projection simply is one of many possible population trajectories, some of which are more likely to occur than others, based on a stochastic model with a number of simplifying assumptions that govern population dynamics. By projecting a large number of trajectories, probability of persistence can be inferred from those trajectory outcomes most likely to occur (that is, extinction versus persistence) while accounting 
for uncertainty caused by stochastic population processes. However, the correct model parameters and assumptions are never known with perfect certainty, and plausible projections under varying parameter values and model assumptions can range from pessimistic to optimistic. The goal was to develop a set of models based on a range of biologically reasonable model parameters and assumptions by which to project population trajectories and characterize persistence probabilities.

To assess the viability of the TRC subpopulation, which was not sampled using the CMR methods, a stochastic age-structured population model was constructed using demographic rates and temporal process variance estimated from the telemetry-based survival and reproduction data. We restricted the population model to age classes $\geq 1$ because data for individual cub survival were insufficient and we wanted to project population dynamics for the same age classes at the TRC as at the TRB and UARB subpopulations. We simulated 10,000 population trajectories over a 100-year period using Monte Carlo methods. The simulation procedure used estimates of adult female survival and temporal process variance from the known-fate survival analysis, stable-state probability for reproductive state $\mathrm{Y}$ and reproductive-state transition probabilities from the multistate transition analysis, and litter-size probability estimates from the multinomial regression analysis of litter counts. Assumptions for the simulation include (1) the sex ratio for yearling litters was $1: 1,(2)$ the age of primiparity for rearing yearling litters was 4 , and (3) the maximum age was 24. Estimates of adult female survival rates and process variances from the known-fate survival analysis were used for all females $\geq 2$ years of age. For yearling survival, estimates were obtained from the published literature on southeastern bears (Hellgren, 1988 [ $S=0.78$ ]; Lombardo, 1993 [ $S=0.53$ ]; Maddrey, 1995 [ $S=0.78$ ]; Beausoleil, 1999 [ $S=0.57]$ ). The mean and sample standard deviation of those estimates to be used in the simulations were then calculated.

To forecast population trajectories using age-structured population models, information about the standing-age distribution is needed to specify the starting conditions for simulations. For bears, this distribution typically is derived from the age distribution of live-captured individuals. However, because live-capture data were not available for the TRC subpopulation, an estimate of the standing age distribution in 2013 could not be made (that is, the starting point of the simulations) using traditional methods. To obtain an initial age distribution, a separate individualbased population model was constructed by simulating annual survival events of censored adult females. Survival events up to 2013 were simulated for female yearlings known to have been produced by radio-collared females in the TRC prior to 2013. Data on unobserved yearlings prior to 2013 were incorporated by simulating the number of yearlings potentially produced by censored females, by females known to be alive through 2013 but lacking known reproductive histories, and by females (known and simulated) that reached reproductive maturity prior to 2013. Data on simulated and known yearlings still alive in 2013 were then combined with simulated and knownalive adult females to define potential standing age distributions in the TRC, which were then used as starting points in the second phase of the population projections.

For the second phase of population projections, life history events (that is, survival and reproduction) in the TRC were simulated for 100 years to project future population trajectories. Multiple approaches were used to assess the manner in which different sources of uncertainty in vital rates affected project population trajectories and inference for population persistence. More specifically, the approaches used addressed uncertainty caused by temporal process variation versus sampling variation, uncertainty in adult survival rates caused by the two ways unknown fates were handled (that is, assumed censored, AC and assumed mortality, AD), and uncertainty in the form and strength of density dependence in reproductive rates. Two-level and three-level approaches were used to incorporate uncertainty caused by process-only variation versus 
uncertainty from process and sampling variation. Because we did not have data-based estimates of process variance for reproductive rates in the TRC, we used coefficients of variation calculated from the estimated means and process variances of $\gamma$ based on the CMR data from the TRB and UARB to derive approximate values of temporal process variation for $R\left(\sigma_{R}\right)$ that reflected observed reproductive variation within the LMAV. Moreover, because we did not have empirical estimates of density-dependent relations between $N$ and reproduction in the TRC, density dependence was incorporated by assuming a relation between $N$ and $R$ on the basis of the Michaelis-Menten function for enzyme kinetics used in Program RISKMAN (Taylor and others, 2006) and defined as

$$
R_{t}=\left(R_{M A X}\right) \frac{\frac{C C}{N_{t-1}}-1}{\frac{C C}{N_{t-1}}-1+\theta},
$$

where $R_{\text {MAX }}$ is the value for $R$ estimated from our reproductive state transition analysis, $C C$ is the carrying capacity for the TRC, and $\theta$ is a shape parameter governing the strength of non-linearity of the density-dependence relation. Because no data were available that could be used to directly determine $C C$ for the TRC, possible values for $C C$ were derived using an estimate of current bear habitat in the TRC and density estimates from the TRB and UARB. To accomplish this, first the amount of current suitable habitat on state or federally owned land or on private land within designated Critical Habitat was quantified (848.4 km² , U.S. Fish and Wildlife Service, 2009) on the basis of habitat classification categories reported by Murrow and others (2013). That value was then multiplied by density estimates for the TRB and UARB derived from abundance estimates based on heterogeneity Model 1 or Model 2 and effective sampling areas calculated by placing a 1,600-m buffer (approximate radius of annual female home range) around respective trapping arrays. By deriving four estimates of $C C$ and simulating separate projections for each, we were able to include uncertainty in population densities that the TRC could support and uncertainty in the most appropriate heterogeneity model used to estimate density. To account for uncertainty in the strength of non-linearity of the density-dependence relation, $\theta=0.1$ and $\theta=0.5$ were considered. Finally, to account for differences in survival rate estimates caused by assuming unknown fates as right censored (AC) or mortalities (AD), simulations were run using both estimates. Combinations of the values of $C C$ and 2 values of $\sigma_{R}$ were restricted to the same population from which those values were derived (that is, $C C$ and $\sigma_{R}$, both from the TRB versus $C C$ and $\sigma_{R}$ from the UARB). In total, 32 combinations of sources of uncertainty were used in the simulations. The outcomes of the 10,000 trajectories for each of those simulations were summarized as for the other two subpopulations.

Population matrix models (Caswell, 2001) were used to estimate the asymptotic rate of population growth $\left(\lambda_{\text {Asym }}\right)$ for the TRB and TRC. Estimates of the number of yearlings per breeding aged female and adult female survival for each study area and estimates of yearling survival obtained from the published literature on southeastern bears were used to parameterize the models. The finite rate of increase module in PopTools (G.M. Hood, 2010; PopTools version 3.2.5 http://www.poptools.org/) was used to calculate deterministic estimates of $\lambda_{\text {Asym }}$.

CMR data were used to conduct viability analyses for TRB and UARB. Six population projections based on combinations of 3 model structures each incorporating different levels of parameter uncertainty and parameter estimates from 2 different capture heterogeneity models (that is, Model 1 and Model 2) were used to evaluate how assumptions about population parameters 
affected our inferences of population viability. For the first projection model structure (hereafter referred to as process-only model), we incorporated environmental (temporal) process variation for $\phi$ and $\gamma$ and included density dependence for $\gamma$ using hyperdistribution parameter estimates obtained from the CMR analysis. Demographic variation was incorporated by using appropriate probability distributions (for example, multinomial and Poisson) to simulate demographic processes. Monte Carlo methods were used to simulate 10,000 trajectories over a 100-year period using a 2-level hierarchical approach:

1. For each year within a simulated trajectory, a random value was drawn for each demographic rate (that is, $\phi$ and $\gamma$ ) from a probability distribution defined by hyperdistribution parameter estimates. Those values were then used to define probability distributions used in the next level. This was done to incorporate temporal process variation in population projections.

2. Within each year, the number of recruits was estimated by drawing a random value from a Poisson distribution with a rate parameter defined as the product of the random value drawn for $\gamma$ and the number of bears alive at the previous time step $\left(N_{t-1}\right)$. The number of survivors was estimated by sampling from a binomial probability distribution defined by the random value drawn for $\phi$ and the sum of $N_{t-1}$ and the previous number of recruits. Those two processes incorporated demographic variation in survival and recruitment into population projections.

The second projection model structure (hereafter collectively referred to as all uncertainty models) incorporated the same sources of variation as the process-only model but also included parameter uncertainty associated with estimates of mean $\phi$ and density-dependence parameters for $\gamma$ (that is, intercept and slope), which required an additional hierarchical level. For each simulated trajectory (that is, level 1 of the simulation process), we first drew a random value from the appropriate set of posterior samples for the hyperdistribution mean of $\phi$ and the intercept and slope parameters that defined the density-dependence relation for $\gamma$. We then used those values and corresponding point estimates of temporal process variance to define the distributions governing temporal variation in vital rates used in the next simulation level (that is, level 2). To avoid drawing extreme and biologically unreasonable values from the tails of the posterior distributions, the posterior distribution of samples was truncated in two ways to assess the effects of incorporating different levels of parameter uncertainty in estimates of persistence probabilities. Specifically, distributions were truncated to the $25 \%$ and $75 \%$ percentiles (that is, 50 -percent credible interval; hereafter referred to as $50 \%$ CI models) and to the $2.5 \%$ and $97.5 \%$ percentiles (that is, 95-percent credible intervals; hereafter referred to as $95 \%$ CI models). Demographic variation in recruitment and survival was incorporated into simulations (that is, level 3), as was done for the process-only model. For all six projections, an upper bound was placed on simulated values of $\gamma$ equal to the second largest annual estimate from each population for each capture heterogeneity model to avoid overly optimistic effects of extremely large values that could be generated by the densitydependence relation if sudden declines in abundance occurred during the simulations. Again, Monte Carlo methods were used to simulate 10,000 population trajectories over a 100-year period.

An estimate of the probability of persistence for each population was derived for each simulation model, based either on CMR or radio-telemetry data, by subtracting the proportion of 10,000 trajectories that became extinct (that is, $N<1$ ) from 1 . Instead of reporting persistence probabilities of 1 , we report probability of persistence estimates of $>0.999$ for simulations where all trajectories remained extant, because such results are based on a finite sample of the distribution of possible trajectories and do not imply absolute certainty of persistence. The ending 
$N$ after 100 years $\left(N_{100}\right)$ was summarized by calculating the mean $\left(\bar{N}_{100}\right), 2.5 \%$ percentile, and $97.5 \%$ percentile for the empirical distribution of $N_{100}$ based on all 10,000 simulations. Population projections and subsequent evaluations of long-term persistence were assumed to apply only to female age classes $\geq 1$. Growth of the LARB population was not projected because only two estimates of interannual parameters were made (for example, $\gamma, \phi$, and $\lambda$ ). Finally, the probability of persistence for the entire system of Louisiana black bear populations was calculated as

$$
1-\prod_{i=1}^{n} 1-\operatorname{Pr}\left(P_{i}\right)
$$

where $\operatorname{Pr}\left(P_{i}\right)$ is the probability of persistence for population $i$ and $n$ is the number of populations. This calculation is based on the assumption that dynamics of individual populations in Louisiana were independent.

\section{Connectivity analysis}

\section{General approach}

The approach for the connectivity analysis was to use genetic data to determine population structure and rates of contemporary exchange between the four subpopulations of Louisiana black bears and to use a step-selection function coupled with global positioning system (GPS) telemetry data to estimate probable movement pathways. The development of highly variable genetic markers, such as microsatellites, enables the direct estimation of movement rates and connectivity among populations from genetic data by differentiating immigrants from residents using individual-level genetic methods. Moreover, such markers permit detection of genetic structure within populations that may reveal the existence of natural- or anthropogenic-caused genetic discontinuities operating at smaller spatial scales that could affect local population dynamics.

Although genetic methods can provide straightforward estimates of interchange rates of individuals and genes, the factors on the landscape that may affect those processes are more difficult to quantify. Furthermore, genetic data by themselves cannot precisely identify where corridors for interchange occur or at what level of functionality those pathways exist. When attempting to identify landscape features that facilitate or impede interchange and dispersal, some type of animal movement data must be incorporated. Radio collars that utilize GPS technology enable the collection of frequent, accurate location estimates throughout the 24-hour period that can be used to determine how landscape attributes affect animal movements and quantify potential connectivity among populations. Thus, genetics data were coupled with GPS data to evaluate connectivity and interchange among the bear subpopulations in our study.

\section{Population Structure Analysis from Genetic Data}

To conduct the genetic analyses, microsatellite data collected from all study populations and associated populations of interest were used to identify groups of similar genotypes at the landscape scale. Potential causes for genetic discontinuities were then identified by comparing clusters of individuals with spatial locations of those clusters and identifying characteristics of the landscape in between. Because inter-population signals of genetic discontinuities between subpopulations can mask signals of intra-population structure, individual analyses for each 
subpopulation also were conducted to search for fine-scale structural patterns at the local population level.

Numerous methods are available to evaluate rates of contemporary and historical gene exchange between animal subpopulations (Spear and others, 2010). For example, genetic assignment tests identify putative migrants as individuals with genotypes that do not fit within an expected genotype distribution and provide estimates of interchange more reflective of current gene flow because they include non-effective dispersal and require fewer assumptions (for example, Hardy-Weinberg equilibrium). Assignment tests are somewhat equivalent to quantifying bear dispersal on the basis of demographic methods (for example, radio telemetry; Manel and others, 2005) because first-generation dispersers can be distinguished from offspring and relatives. In addition, natural or anthropogenic restrictions of gene flow between adjacent areas of occupied habitat can be revealed with genetic structuring. Genetic distance measures are commonly calculated between groups of individuals where group membership is based on subjective criteria and used to identify landscape features associated with reduced gene flow. Because factors causing zones of restricted movement may be cryptic or associated with unknown landscape characteristics, subjective criteria for assigning group membership may result in biased inference when identifying locations and causes of restricted gene flow. A more robust approach for delineating groups is to assume there is no previously existing structure and allow the genetic data to objectively identify clusters.

Adequate power to identify immigrants requires genotyping a sufficient number of individual bears from each population or population segment (for example, each side of a potential discontinuity) and using a sufficient number of markers. To ensure adequate power to detect migrants and genetic discontinuities, genotypes from 50 or more individuals per potential population segment were extended beyond the number of markers used for CMR to 23 markers. Potential population segments in the TRB were investigated, which required genotyping additional markers for $\geq 100$ individuals. Likewise, genotypes for $\geq 100$ individuals in the LARB were extended to the same 23 markers to investigate potential genetic structure within that population. Finally, genotypes for $\geq 50$ individuals in the UARB were extended to 23 markers to investigate regional genetic structure among the TRB, UARB, and LARB. Microsatellite genotype data collected from bear populations in central Itasca County, Minnesota (MINN), the White River Basin of Arkansas (WRB), the TRC, and western and southern Mississippi (MISS) were used. Bears from Minnesota were reintroduced to the TRB and UARB, so we wanted to determine the amount of influence those bears may still have on the study populations in Louisiana. Also, bears from the WRB may be closely related to Louisiana black bears and bears from the WRB were reintroduced to Felsenthal National Wildlife Refuge (FNWR) in south central Arkansas (Wear and others, 2005), approximately $200 \mathrm{~km}$ northwest of TRB. We wanted to evaluate possible gene exchange between WRB bears and bears in Louisiana. Finally, a growing bear population is present in western Mississippi, and we were interested in examining the source of those bears to provide further insight on movement and dispersal potential.

Two multivariate clustering methods, spatial distribution of bears in the LMAV, and knowledge of reintroduction history in Louisiana were used to determine population structure and identify genetically distinct populations that would be used in subsequent migrant identification analyses. The first clustering method was factorial correspondence analysis (FCA; She and others, 1987) in Program GENETIX (Belkhir, 2004). FCA is a special case of principal components analysis that uses multivariate categorical data to identify structural relations among variables without any previous information or expectations, such as the true number of clusters. For this 
analysis, FCA was used to reduce landscape-level multi-locus genotype data from all or specific subsets of populations down to principal dimensions from which groups of individuals with similar genotypes could be identified using graphical displays. A visual comparison was made of the distribution of individuals among inferred populations to the true spatial distribution of those individuals across the landscape to infer the appropriate number and juxtaposition of distinct populations to be used in migrant analyses. Furthermore, because inter-population signals of genetic discontinuities between subpopulations can mask signals of intra-population structure, separate FCA analyses were conducted for each of the four subpopulations in Louisiana to search for fine-scale structural patterns at the population level and identify potential movement barriers within populations.

The second clustering method was a model-based clustering algorithm that infers population structure by assuming a user-specified number of populations $(K)$ employed by Program STRUCTURE (Pritchard and others, 2000). Each putative population is assumed to be characterized by a unique set of allele frequencies and loci in Hardy-Weinberg equilibrium and linkage equilibrium within populations. Because this method is conditional on $K$, multiple values of $K$ need to be evaluated. To choose the most likely number of populations occurring within the genetic dataset, models were fitted that assumed different values of $K$ ranging from 2 to 11 , and the model was selected that best described the data on the basis of the log of the posterior probability of the data for a given $K(\log [K]$; Pritchard and others, 2000), the second-order rate of change of $\log [K](\Delta \log [K]$, Evanno and others, 2005), and prior knowledge of the historical and current distribution of bear populations within the LMAV. Individual admixture was included in the model to estimate the probability of an individual coming from each of the putative clusters, and it was assumed that allele frequencies were correlated because extant bear populations along the LMAV historically were a continuous single population. Program STRUCTURE employs Bayesian methods and MCMC sampling to generate samples from the posterior distribution from which parameter estimates can be obtained and the most appropriate value of $K$ can be inferred. Ten independent chains were run in a simulation for each value of $K$ to account for among-chain variation in convergence. Each chain was run for 500,000 steps, whereby the first 50,000 samples were discarded as burn-in, and the subsequent 450,000 samples were retained for inference. On the basis of those results, the most likely value of $K$ was selected and 10 additional chains with 500,000 burn-in samples and 500,000 retained samples were run to ensure consistent and reliable convergence across chains.

\section{Migrant Analysis Based on Genetic Data}

Two independent assignment methods were used to identify putative first-generation migrants between all pairs of population units identified by FCA and Program STRUCTURE analyses. Analysis was limited to bears from the WRB, TRB, UARB, and LARB because natural movement between MINN and any of the other areas sampled was not possible. First, individuals were assigned to populations of origin with the highest probability on the basis of populationspecific allele frequencies using simulation methods for distinguishing true migrants (Paetkau and others, 2004) available in the software package Program GeneClass 2.0 (Piry and others, 2004). Migrants were identified as those bears with assigned population that differed from the population in which they were sampled. The ratio of the likelihood of an individual's genotype coming from its sample population to the highest likelihood of that genotype coming from any of the sampled populations was used as the test statistic for determining significance. To obtain critical values for determining migrant status of each individual, we used the empirical distribution of test statistic 
values calculated from 10,000 simulated genotypes generated using observed allele frequencies of an individual's sample population. Migrant status was assigned to bears if their test statistics fell beyond the $2.5 \%$ and $97.5 \%$ percentiles (that is, Type I error rate of the test statistic distribution). To account for missing alleles, allele frequencies were set to 0.01 in populations where specific alleles were not observed.

Next, putative migrants were independently tested for using the model-based approach available in Program STRUCTURE (Pritchard and others, 2000). The assignment method in Program STRUCTURE is similar to the approach used to identify population clusters, except that prior population information is directly incorporated into the analysis. This is accomplished by coding each population with an integer value between 1 and $K$, where $K$ is the number of populations identified in the previous analysis, and assigning each individual with the value of $K$ pertaining to the population in which it was sampled. Using this model, the probability can be estimated that an individual is an immigrant to its sampled population or is an $n$th generation offspring of a migrant ancestor (Pritchard and others, 2000). Allele frequencies were assumed to be correlated among populations, and the value was set for the assumed migration rate (required user-specified input) to 0.01 because it was assumed that rates of interchange were low among such highly fragmented populations. A single chain of 200,000 steps was run, discarding the first 100,000 samples as burn-in and retaining the remaining 100,000 for inference. To obtain conservative estimates of interchange, migrants were defined as individuals that were classified as putative migrants by both assignment methods.

Putative migrants to the TRC could not be directly tested because samples from bears other than translocated females and their offspring were not available. Alternatively, interchange between the TRC and UARB was indirectly assessed by testing cubs born in the TRC to females with known TRB ancestry (that is, translocated to TRC from TRB) for evidence of being sired by males immigrating from the UARB (that is, second generation migrants). Bears sampled in the TRB and TRC cubs were classified as a single population cluster, and that cluster was compared with the UARB cluster using Program STRUCTURE. Program STRUCTURE identifies individuals with recent immigrant ancestry by estimating probabilities that an individual has a direct immigrant ancestor in the past $G$ generations (that is, immigrant parent or grandparent). For TRC cubs, the probability was estimated that each individual had one immigrant parent (that is, father) from the UARB to identify those cubs showing evidence of having been sired by males with UARB ancestry.

To supplement the search for migrants on the basis of genetic assignment tests, DNA-based CMR histories and live-capture records were searched for individuals that occurred in more than one population within the LMAV. Those individuals then were combined with migrants from assignment tests to determine the total number of migrants.

\section{GPS Telemetry Data to Estimate Successful Interchange Rates and Paths}

Approaches for quantifying animal movement processes on the basis of GPS data are in rapid development (Chetkiewicz and others, 2006; Forester and others, 2009; Cagnacci and others, 2010). One method for describing such movement is based on a step-selection function modeling approach (Fortin and others, 2005). Step-selection functions are similar to resource selection functions (Manly and others, 2002) in that an observed movement is compared with a random set of potential movements that were possible but not chosen. If it can be assumed that the step chosen and those not chosen are exclusive, then discrete choice analysis can be employed whereby predictions are true probabilities of use (Manly and others, 2002); if the assumption does not hold, 
then the predictions are relative probabilities of use (McDonald, 2013). Instead of individual points on the landscape as with typical resource selection function models, movement in a step-selection function model is described as a "step" between two sequential radiolocations having a step length and turning angle. Potential steps are generated on the basis of an assumed distribution of step lengths and turning angles, and environmental covariates associated with those (control) are compared with the covariates associated with the observed step (case). Conditional (case-control) logistic regression is then used to fit a linear model to the data on the logit scale (Hosmer and Lemeshow, 1989). Thus, step-selection function analyses can be used to identify landscape attributes associated with the movement from one point in space and time to another (Fortin and others, 2005, Chetkiewicz and others, 2006). On the basis of such models, movement pathways can be projected on the landscape to determine where animals are likely to travel and the relative frequency that particular routes are chosen. Thus, probable movement pathways can be identified and potential movement extents can be quantified. Thus the goal was to develop a step-selection function to simulate movement paths to quantify potential successful dispersal events and to compare potential interchange rates with rates of gene exchange among the four subpopulations of Louisiana black bears.

Black bears exhibit male-biased dispersal (Schwartz and Franzmann, 1992; Costello and others, 2008) so radio-tagging activities were focused on young males, which were more likely than other bears to exhibit long-range movements. Female dispersal can also be important for connectivity and demographic rescue (Onorato and Hellgren, 2001), and the factors responsible for female movement on the landscape may differ from those for males. Therefore, an attempt was made to trap and radio collar a small sample of females as well. Bears were captured in culvert traps, immobilized, and equipped with store-on-board GPS radio collars (Telonics, Mesa, Arizona; North Star Science and Technology, King George, Virginia) programmed to obtain one radiolocation per 2-4 hours; collar batteries were expected to last about 1.5 years. Collars were equipped with remote detachment mechanisms that were programmed to release prior to the end of their anticipated battery life or the end of the study period. Data from recovered collars were then downloaded and stored in a spreadsheet database. In addition to the store-on-board function, data from the North Star collars could be uploaded via satellite. Uploading was programmed to take place concurrently with location acquisition, although intermittent satellite availability prevented uploading of all locations that were stored on board the collars. The potentially reduced satellite datasets were used only when the collars were not retrievable for direct downloading.

All GPS locations with dilution of precision (PDOP) >7 (Lewis and others, 2007) and locations obtained during the denning season were omitted from the analysis. The denning season was defined as November 15 to April 15 for females and December 15 to April 1 for males, loosely based on the work by Waller and others (2012). Sequential locations $<100 \mathrm{~m}$ apart were deleted because the point of interest was in characterizing movements of bears that were actively moving (Latham and others, 2011). Because collars were programmed to collect location data at 2-, 3-, or 4-hour intervals, all steps consisting of location parings <4.1 hours apart were included; 0.1 hour was added to include locations that were on the margin. To determine whether step length was correlated with turning angles, an angular-linear correlation coefficient was calculated (Fisher, 1995).

Geospatial Modeling Environment (GME) software (Beyer, 2012) was used to separately estimate step lengths and turning angles for males and females. To characterize step lengths, the observed step lengths were binned into 24 categories, ranging from $100 \mathrm{~m}$ to 10,000 m. Observed turning angles were grouped into eighteen 20-degree bins. Potential step length and turning angle 
distributions for each bear were based on the empirical data from all the other radio tagged bears of the same sex. On the basis of these distributions, 10 random steps were created for every observed step for each bear in the study.

A variety of spatial data was used to characterize landscape attributes thought to be associated with movement steps in the step-selection models. For land cover, the National Land Cover Database 2006 (NLCD 2006; http://www.mrlc.gov) was used, which is a 16-class land cover classification scheme. NLCD 2006 has a spatial resolution of $30 \mathrm{~m}$ and is primarily based on the unsupervised classification of Landsat Enhanced Thematic Mapper+ circa 2006 satellite data (Fry and others, 2011). Land cover data were simplified by creating a mast-producing forest category composed of deciduous forest, mixed forest, and woody wetlands using ArcMap ${ }^{\circledR} 10.1$ Geographic Information System (GIS; Environmental Systems Research Institute, Redlands, California). Those cover types have been shown to be important to Louisiana black bears (Nyland, 1995; Benson and Chamberlain, 2007; Murrow and Clark, 2012). The non-forest category represented all other land cover types, including urban, agricultural, and barren areas. Data layers were created consisting of the Euclidian distance to nearest forest and percentage of forest as was a data layer of forest density on the basis of a neighborhood analysis of the mast-producing forest category using a 150 $\mathrm{m} \times 150 \mathrm{~m}$ focal area. Because other natural cover types could be used by bears, we created a "natural” variable, which consisted of deciduous forest, evergreen forest, mixed forest, shrub/scrub, grassland/herbaceous, and woody wetlands. The natural data layer included Conservation Reserve Program and Wetland Reserve Program data provided by the USFWS, which were coded as natural. We created a distance to natural and percent natural data layers. A natural density data layer was also created based on a larger focal area $(1,500 \mathrm{~m} \times 1,500 \mathrm{~m})$ to characterize land cover at a different scale. Using the NLCD 2006 data, two agriculture variables were created: percent agriculture and Euclidean distance to agriculture. The land cover maps did not accurately show forests available to bears because much of the woody wetlands cover type identified by Landsat was flooded and not used by bears. Therefore, we downloaded flood model data (http://abp.cr.usgs.gov/Library/Default.aspx?folder=268) and the map showing the mean gage level at Butte La Rose, Louisiana (http://waterdata.usgs.gov/nwis/ inventory/?site_no=07381515\&agency_cd=USGS), which we determined to best coincide with the 14.6-ft (4.45-m) map. That layer was used to reclassify the forest and natural cover maps so that only non-flooded areas remained. Open water was classified as a single cover type from the land cover map, and the Euclidean distance to the nearest water was calculated on the basis of that reclassification.

Fragstats software (McGarigal and Marks, 1995; Haines-Young and Chopping, 1996; Turner and others, 2001) was used to calculate three variables (that is, contagion, splitting index, and landscape shape index) to quantify broad-scale patterns of forest/wetland edge and patch configuration. Contagion is a measure of both the spatial distribution and intermixing of patch types and is inversely related to edge density (McGarigal and Marks, 1995). Splitting index is a measure of the patchiness of the landscape; the index increases as the landscape is subdivided into smaller patches. Landscape shape index is a measure of landscape shape, including land-cover aggregation.

Road information was obtained from 2012 TIGER/Line ${ }^{\circledR}$ data that were based on the redistricting 2010 census (U.S. Bureau of the Census, 2012). Two road variables were created to address human impacts and fragmentation of the landscape. Road density was calculated on the basis of all roads (including minor, unpaved roads). Road density was not calculated for areas 
outside the boundaries of Louisiana because road definitions vary by state. The Euclidean distance to roads was calculated from that same dataset.

Following the creation of the spatial variables, the GME tool isectlinerst was used to combine the observed and potential steps with the landscape data. For each landscape variable and step, five summary attributes were assigned: the value at the beginning of a step, the value at the end of a step, the maximum value, the minimum value, and the linear weighted mean along the step. To estimate the step-selection function for each sex, we used conditional logistic regression in Program R 2.15.3 (R Core Team, 2013), which is based on a Cox proportional hazards model in the survival package (Therneau, 2013). First, a correlation analysis was performed, excluding one variable of any pair of correlated variables (that is, Pearson's $r>0.6$ ). The list of possible variables was lengthy, so a data dredging procedure to fit all possible models was not feasible. Instead, individual main effects were fitted first following methods of Hosmer and Lemeshow (1989). To avoid redundancy, only 1 of the 5 attributes (beginning, end, minimum, maximum, or mean) for each landscape variable was included, based on the attribute that had the lowest Akaike Information Criterion score (AIC; Burnham and Anderson, 2002). Following that, main effects were added and removed and interactions and quadratic effects, based on a combination of AIC scores and the stability and interpretability of individual coefficients (Compton and others, 2002), were evaluated. We also wanted to evaluate whether agriculture would be used differently by bears during the growing season when crops provided some food and hiding cover. Therefore, the growing season (between June and November) and the non-growing season were modeled as interaction terms with distance to agriculture. Finally, mixed effects models were fitted by modeling deviations of individual bears $\left(b_{i}\right)$ from the mean response $(\beta)$ as $\beta+b_{i}$, where $b_{i} \sim \operatorname{Normal}\left(0, \sigma^{2}\right)$ and $\sigma^{2}$ was the variance term describing the dispersion across individuals (Duchesne and others, 2010). We did this to determine whether effects of major landscape variables differed by individual.

Autocorrelation among sequential spatial data can produce biased estimates of variance (Nielsen and others, 2002). Therefore, robust standard errors (SE) for fitted parameters were estimated from fixed effect models on the basis of work by Fortin and others (2005) and Forester and others (2009). Then 95-percent CIs were created for the parameter estimates based on those robust SEs, and those parameters from the top model were excluded if the interval included zero. Conventional SEs for mixed effects models were reported because robust estimates were not available.

The GME simulation tool movement.ssfsim 1 was used to simulate correlated random walks (Turchin, 1998) of bears from each of the subpopulations on the basis of the fixed-effects step-selection function for each sex. No attempt was made to simulate paths on the basis of the mixed effects models. The simulation tool functioned by beginning at a starting point and then choosing a random bearing associated with that point. Next, the tool generated a number of available steps $(n=10)$ based on our empirical distributions of step lengths and turning angles after which the likelihood of each step was calculated on the basis of the landscape covariates and our linear step-selection function. Finally, a single step was selected, and the process continued for a pre-defined number of steps and replications; the probability of a particular step being selected was proportional to its likelihood. Steps for females and males were projected to be 1,895 and 2,236, respectively, which represents the number of expected GPS fix intervals during the nondenning season per year (that is, hours during the non-denning season divided by the mean fix interval). One hundred random locations within each subpopulation were used as starting points, and 40 replicate projections of each were created (that is, 4,000 paths). Then the proportion of 
paths that intersected adjacent subpopulations, defined as the successful interchange rate, was calculated.

To evaluate the effects of corridors on successful interchange rates, hypothetical 1,000-mand 3,000-m-wide corridors were created, consisting of natural cover types, between each of the closest pairings of the four subpopulations. Then the path simulation routine was run to evaluate the effect of the potential corridors on successful interchange rates. Finally, the step-selection function was applied to the landscape in ArcMap, based on a logistic transformation of the linear equations. Then the inverse of those values, which was defined as relative affinity (cost) for traveling through a given 30-m pixel (Chetkiewicz and Boyce, 2009), was calculated.

Finally, to evaluate the worst-case scenario for long-term protection of Louisiana black bear habitat and movement pathways, all natural areas were excluded from the analysis, except U.S. Fish and Wildlife Service National Wildlife Refuges and Partners for Fish and Wildlife Lands, U.S. National Forest Land, U.S. Department of Agriculture Wetland Resources Protection Land, U.S. Army Corps of Engineers Lands, Tribal Lands, Nature Conservancy Lands, National Park Lands, Mitigation Bank Lands, Louisiana State Wildlife Management Areas, Farmers Home Administration lands, Ducks Unlimited Conservation Easement Lands, and Bureau of Land Management Lands (Andy Dolan, USFWS, unpublished data). The distance to natural data layer was based on natural cover types that were considered to be permanently protected, and the analysis was rerun to compare successful interchange rates to the results of previous analyses that were based on currently available land cover.

\section{Results}

\section{Population Viability Analysis}

\section{Survival Rates of Radio-Collared Adult Female Bears}

From 2002 to 2012, 86 radio-collared adult females $>2$ years old were monitored within the TRB, representing 305 bear years, and in the TRC, 43 adult females were monitored, representing 208 bear years. Four and 9 known mortalities in the TRB and TRC, respectively, were recorded. Causes of known mortalities in the TRB were collisions with automobiles $(n=2)$, research related $(n=1)$, and unknown $(n=1)$. In the TRC, mortalities were caused by automobile collisions $(n=$ $3)$, illegal kills $(n=4)$, natural $(n=1)$, and research related $(n=1)$. When we assumed unknown fates were mortalities (assumed dead, AD), we documented 10 and 16 mortalities in the TRB and TRC, respectively.

When unknown fates were right censored (AC), the mean annual survival rate estimate was 0.99 (95\% CI = 0.96-1.00) for TRB and 0.97 (95\% CI = 0.91-0.99) for TRC (fig. 2). Assuming unknown fates were mortalities (AD), mean annual survival rates were $0.97(95 \% \mathrm{CI}=0.93-0.99)$ in the TRB and $0.93(95 \% \mathrm{CI}=0.85-0.97)$ in the TRC (fig. 2). In general, annual survival rate estimates were higher and less variable for the TRB than for the TRC, regardless of censoring method (figs. 3-4). 
A

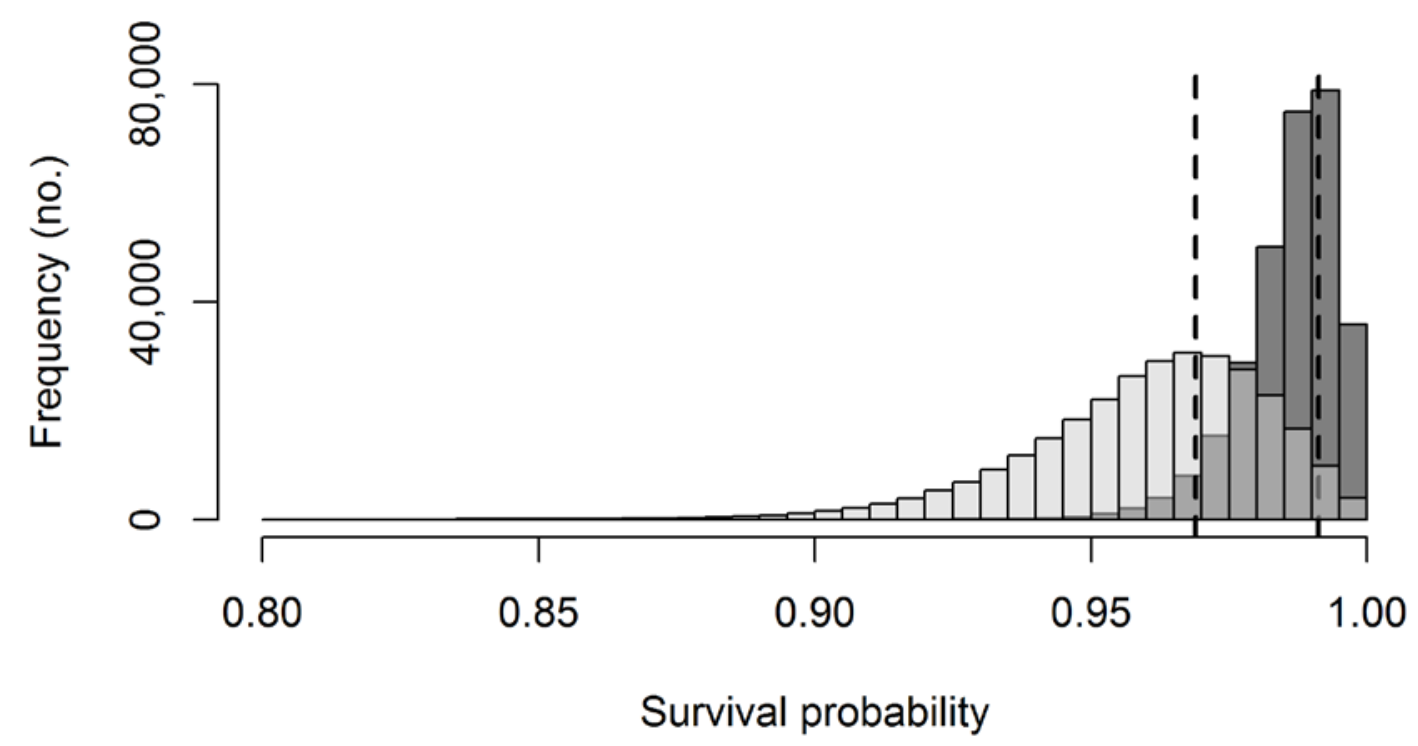

B

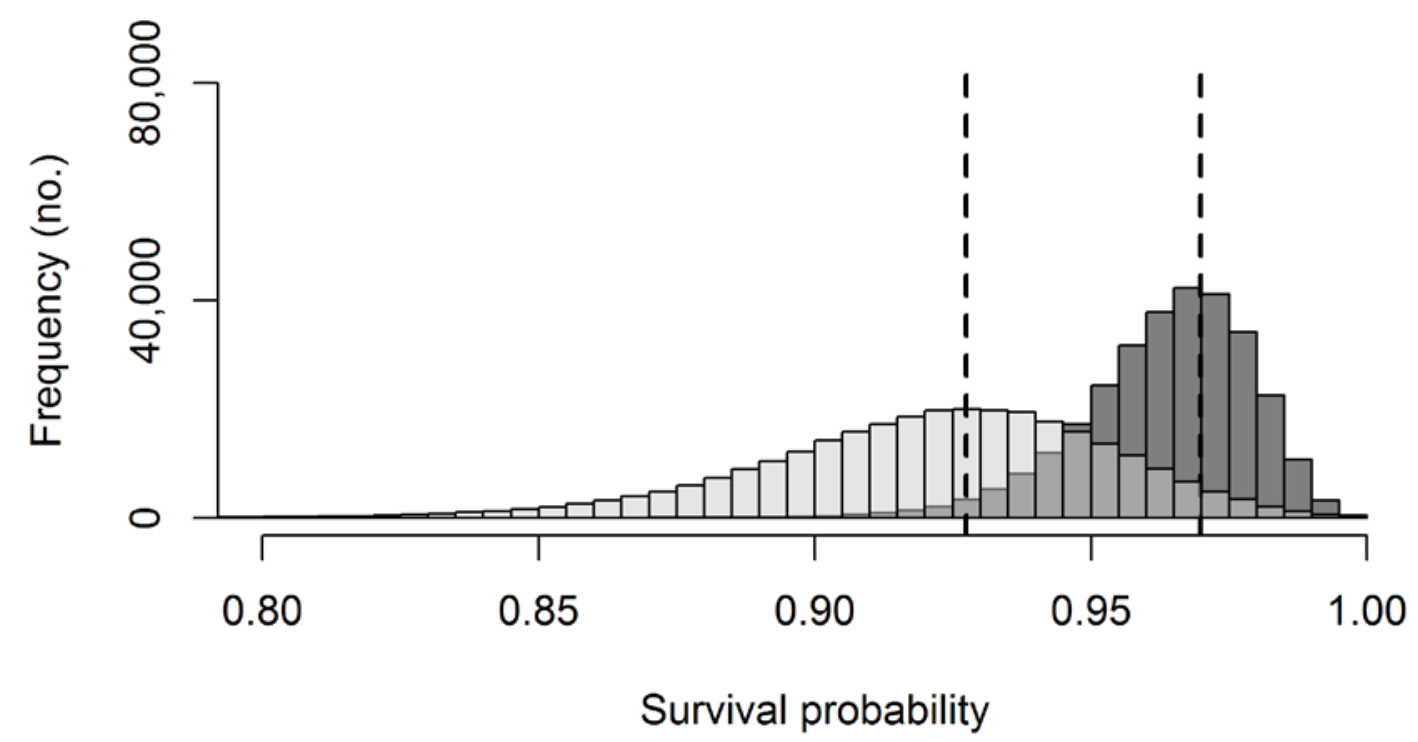

Figure 2. Posterior distributions of assumed-alive $A$, and assumed-dead $B$, mean annual adult female survival estimates for Louisiana black bears within the Three Rivers Complex (light gray) and Tensas River Basin (dark gray) in Louisiana, 2002-12. Dashed lines are posterior distribution modes. (no., number). 


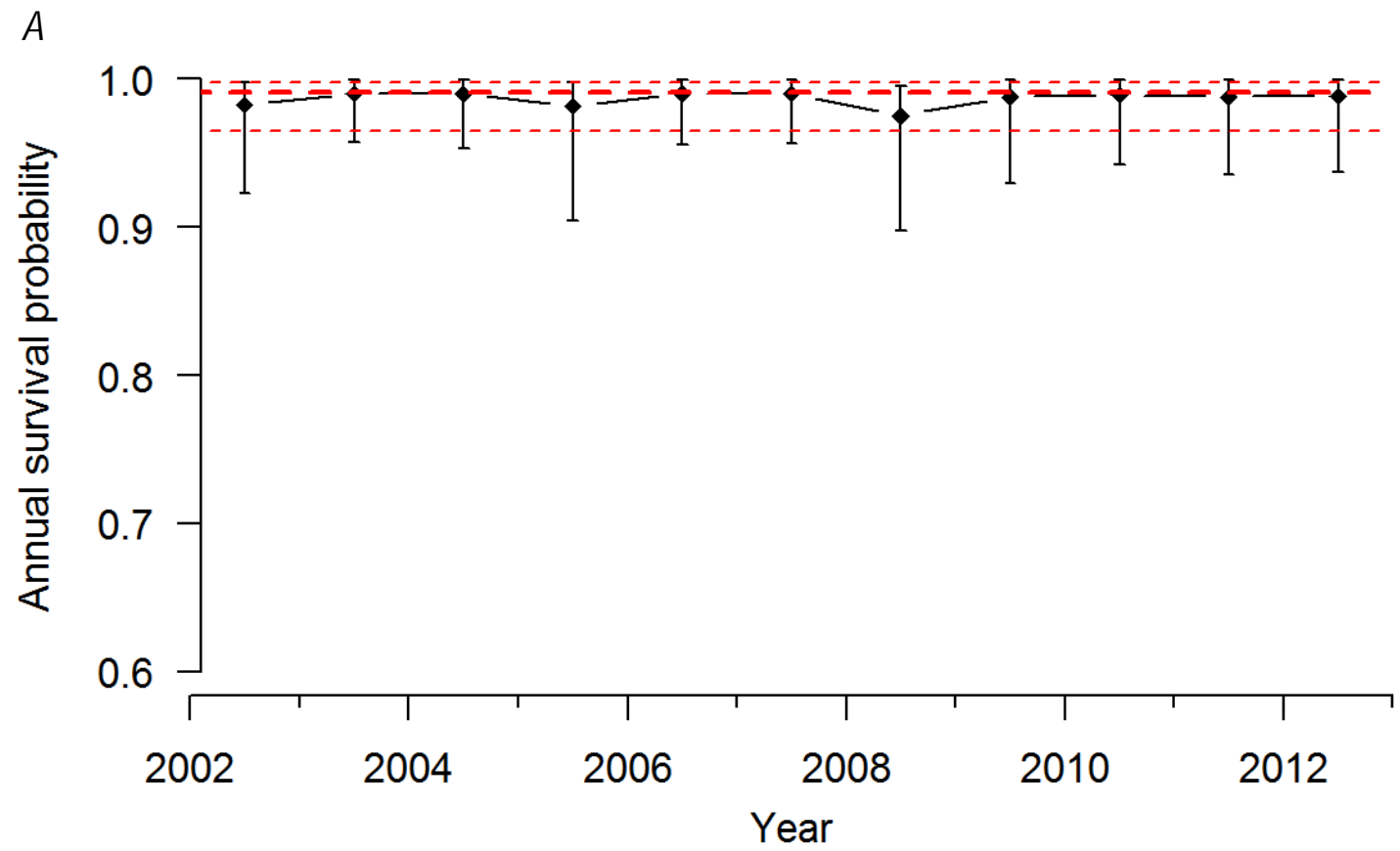

$B$

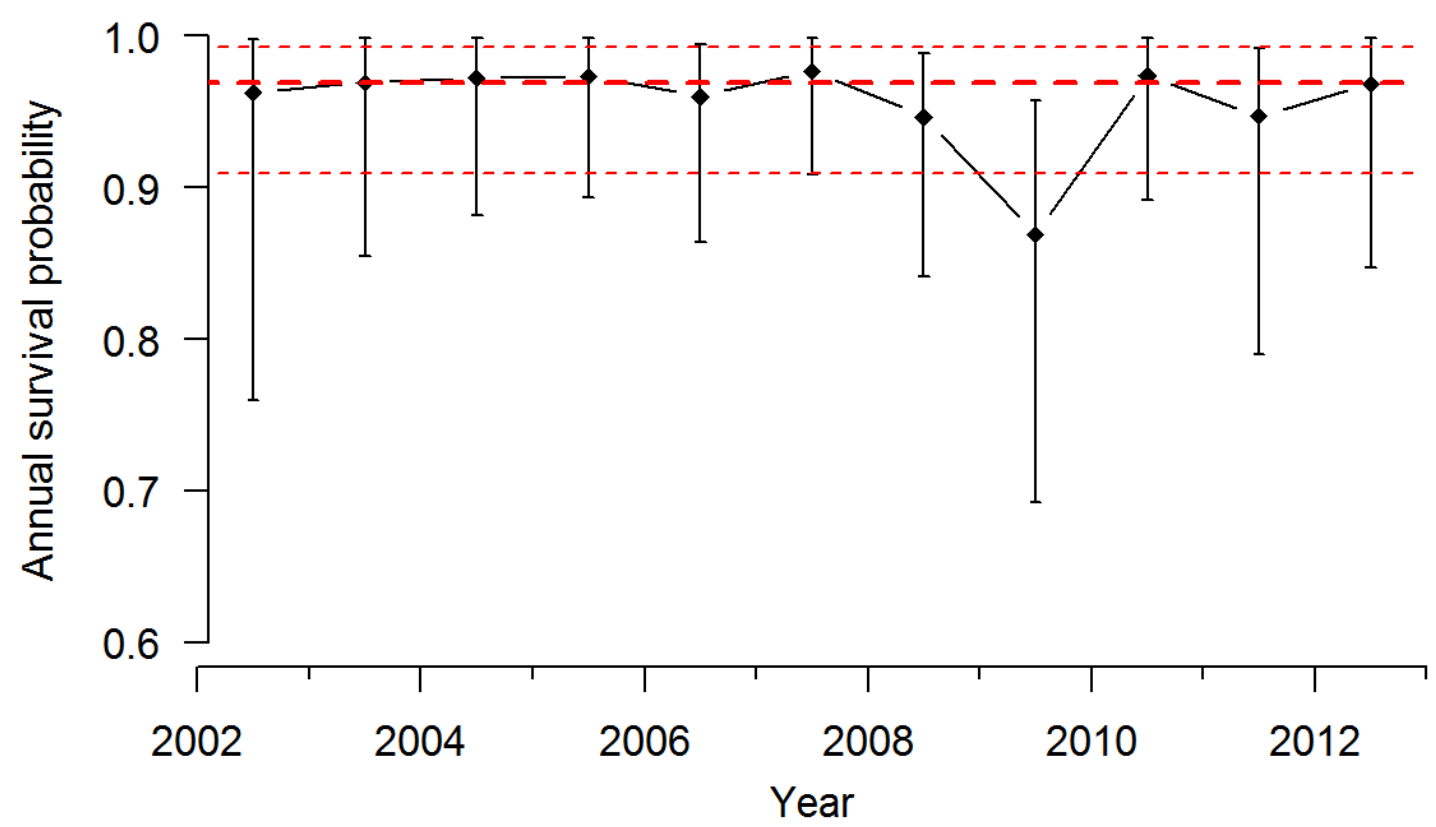

Figure 3. Annual estimates (diamonds) and 95-percent credible intervals (bars) of adult female survival for Louisiana black bears within the Tensas River Basin A, and Three Rivers Complex B, in Louisiana, 2002-12. For the estimates, it was assumed that bears with unresolved fates were alive at time of last contact. Thick dashed lines are mean annual survival estimates and thin dashed lines are 95-percent credible intervals. 

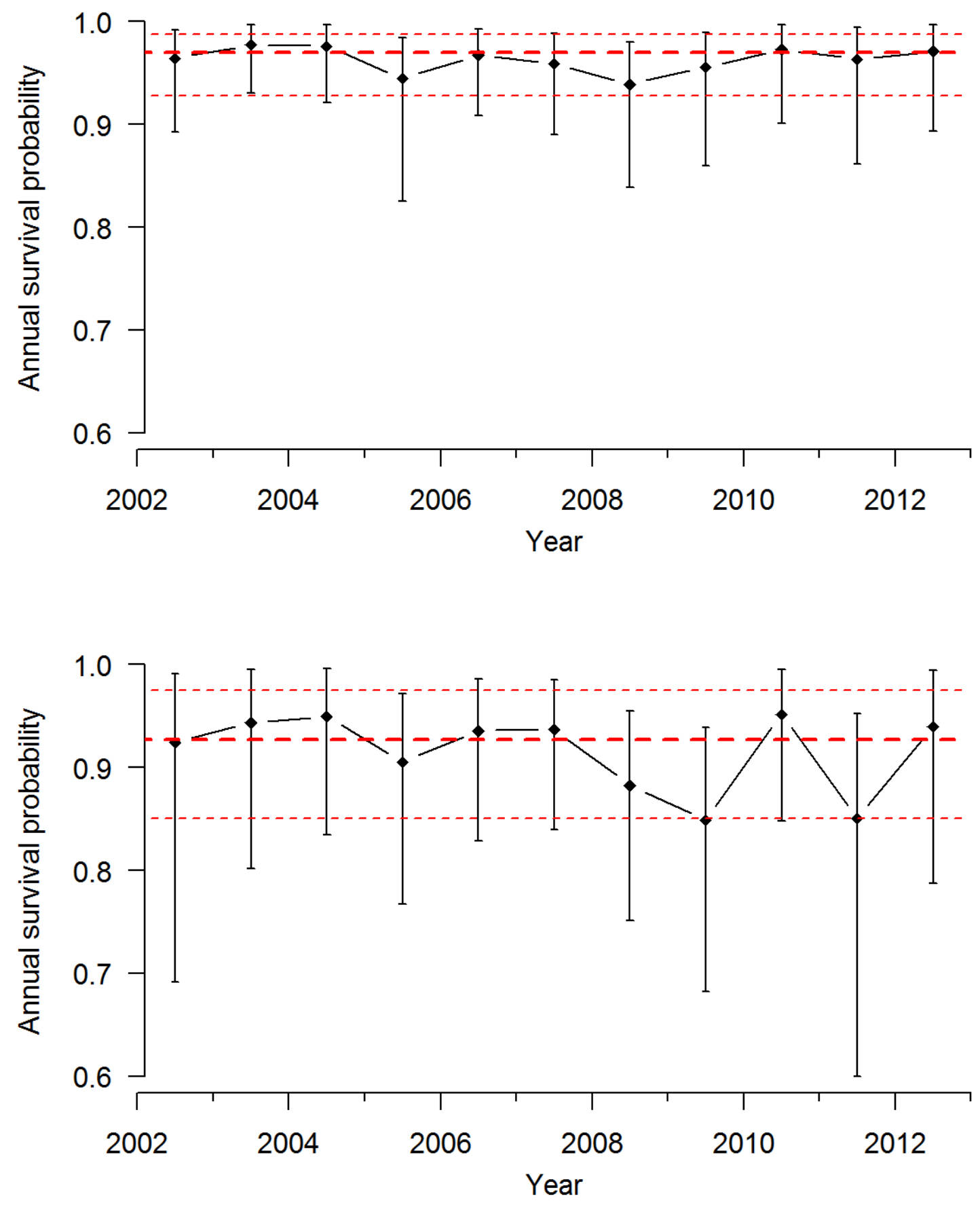

Figure 4. Annual estimates (diamonds) and 95-percent credible intervals (error bars) of adult female survival for Louisiana black bears within the Tensas River Basin $A$, and Three Rivers Complex $B$, in Louisiana, 2002-12. For the estimates, it was assumed that bears with unresolved fates were dead at time of last contact. Thick dashed lines are mean annual survival estimates and thin dashed lines are 95-percent credible intervals. 


\section{Reproductive Rates of Radio-Collared Adult Female Bears}

From 2003 to 2013, 142 transitions were observed among reproductive states for 58 females in TRB and 74 transitions for 29 females in TRC. Females in the TRB were more likely to transition to state $\mathrm{C}$ from any previous state (that is, $\psi_{i, 2}$ for $i=1,2,3$ ) than females in TRC (table 3). In contrast, females in TRC were more likely to transition from state $\mathrm{C}$ to state $\mathrm{Y}$ (that is, $\psi_{2,3}$ ) than females in TRB. Furthermore, females in TRC were nearly twice as likely to remain in state B $\left(\psi_{1,1}=0.67,95 \%\right.$ credible interval $\left.=0.46-0.82\right)$ than females in TRB $\left(\psi_{1,1}=0.347,95 \% \mathrm{CI}=\right.$ 0.23-0.46). The estimated stable state probability of females being in state B was greater in TRC $(\operatorname{Pr}(\mathrm{B})=0.47,95 \% \mathrm{CI}=0.31-0.64)$ than in $\mathrm{TRB}(\operatorname{Pr}(\mathrm{B})=0.27,95 \% \mathrm{CI}=0.19-0.36$; fig. 5), whereas the estimated probability of females being in state $C$ was greater in $\operatorname{TRB}(\operatorname{Pr}(\mathrm{C})=0.51$, $95 \% \mathrm{CI}=0.45-0.57)$ than in the TRC $(\operatorname{Pr}(\mathrm{C})=0.34,95 \% \mathrm{CI}=0.23-0.43)$. The proportion of females in state $\mathrm{Y}$ was nearly identical for $\mathrm{TRB}(\operatorname{Pr}(\mathrm{Y})=0.22,95 \% \mathrm{CI}=0.16-0.28)$ and $\mathrm{TRC}$ $(\operatorname{Pr}(\mathrm{Y})=0.19,95 \% \mathrm{CI}=0.12-0.27)$.

Table 3. Estimated transition rates between reproductive states for adult female Louisiana black bears in the Tensas River Basin (TRB) and Three Rivers Complex (TRC), Louisiana, 2002-12.

[Values in parentheses are 95-percent credible intervals. B, barren (without cubs or yearlings); C, with cubs; Y, with yearlings]

\begin{tabular}{|c|c|c|c|}
\hline \multicolumn{4}{|c|}{ Probability of transitioning to reproductive state } \\
\hline Current reproductive state & B & $\mathrm{C}$ & $\mathbf{Y}$ \\
\hline \multicolumn{4}{|c|}{ TRB } \\
\hline B & $0.34(0.23-0.46)$ & $0.66(0.54-0.77)$ & 0 \\
\hline $\mathrm{C}$ & $0.25(0.16-0.38)$ & $0.31(0.20-0.43)$ & $0.43(0.31-0.56)$ \\
\hline $\mathrm{Y}$ & $0.20(0.09-0.39)$ & $0.80(0.61-0.91)$ & 0 \\
\hline \multicolumn{4}{|c|}{ TRC } \\
\hline B & $0.67(0.46-0.82)$ & $0.33(0.18-0.54)$ & 0 \\
\hline $\mathrm{C}$ & $0.26(0.14-0.41)$ & $0.14(0.06-0.29)$ & $0.58(0.42-0.73)$ \\
\hline $\mathrm{Y}$ & $0.33(0.15-0.59)$ & $0.67(0.41-0.85)$ & 0 \\
\hline
\end{tabular}

From 2003 to 2013, 130 cub litters were observed for 74 females in the TRB, and 74 cub litters were observed for 45 females in the TRC. During the same period, 43 litters consisting of yearlings for 33 females were observed in the TRB, and 21 yearling litters were observed for 19 females in the TRC. Although estimated probabilities of females having litters of 1 or 2 cubs were greater in the TRB than in the TRC and probability estimates for 3- or 4-cub litters were greater in the TRC, strong evidence of a true difference existed only for the 3-cub litter category (that is, minimal overlap of 95\% CIs; fig. 6). Similarly, females in the TRB were more likely to have single-yearling litters, and females in the TRC were more likely to have 2- and 4-yearling litters, although there was substantial overlap among 95-percent credible intervals. Mean cub and yearling litter sizes were $1.85(95 \% \mathrm{CI}=1.72-1.99)$ and $1.40(95 \% \mathrm{CI}=1.26-1.64)$ in the TRB, respectively, whereas estimates for the TRC were $2.15(95 \% \mathrm{CI}=1.94-2.37)$ and $1.84(95 \% \mathrm{CI}=$ 1.55-2.28), respectively. Estimates of $f$ and $r$ for the TRB were 0.47 (95\% CI $=0.41-0.54)$ and $0.15(95 \% \mathrm{CI}=0.11-0.20)$, respectively, whereas estimates for the TRC were 0.37 (95\% CI = $0.25-0.47)$ and 0.18 (95\% CI $=0.11-0.27)$, respectively. 

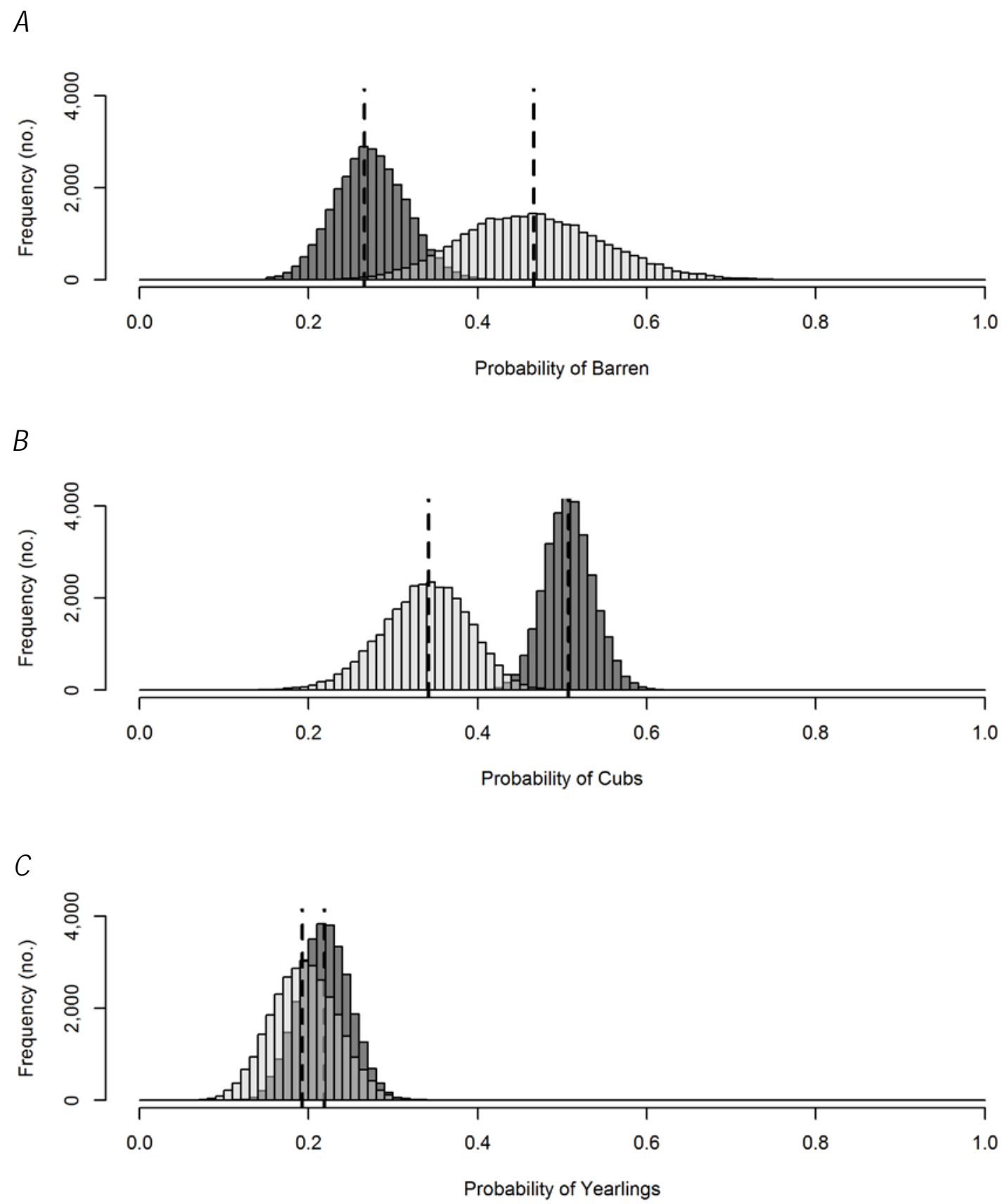

Figure 5. Posterior distributions for proportions of adult female Louisiana black bears with no litters $A$, cubs $B$, and yearlings $C$, within the Tensas River Basin (light gray) and Three Rivers Complex (dark gray) in Louisiana, 2002-12. Dashed lines are posterior distribution modes. (no., number). 

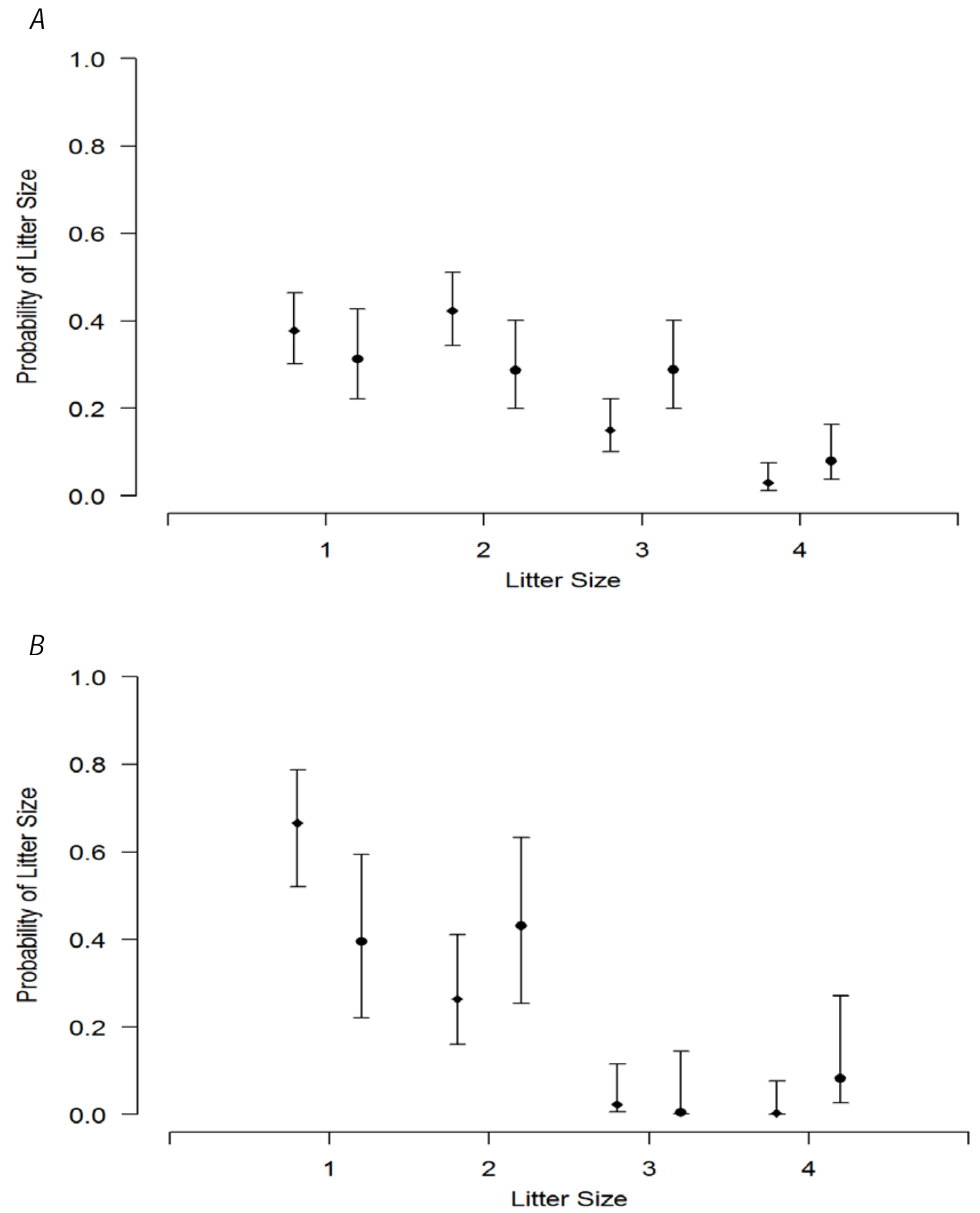

Figure 6. Estimated litter size probabilities (diamonds and circles) of cub $A$, and yearling $B$, litters and 95percent credible intervals (error bars) for adult female Louisiana black bears within the Tensas River Basin (diamonds) and Three Rivers Complex (circles) in Louisiana, 2002-12.

Demographic Rates from Capture-Mark-Recapture Data

From 2006 to 2012, 23,312 hair samples were collected in the TRB. The weekly number of sites that produced one or more viable hair samples ranged from 35 to 174 and the weekly number of samples collected ranged from 98 to 1,382. Of the 209 sites surveyed each year in the TRB, the annual number 
of sites that produced one or more collected hair samples across all weeks ranged from 138 to206. In the UARB, from 15 to 607 samples were collected each week, and a total of 11,643 samples were collected from 2007 to 2012. Of the 115 sites surveyed each year in the UARB, the annual number of sites that produced one or more collected hair samples ranged from 65 to 115 . The number of sites each week from which samples were collected in the LARB ranged from 7 to 101. From 2010 to 2012, the number of sites producing samples in the LARB varied from 26 to 78. The number of samples collected each week ranged from 53 to 281, totaling 3,698 samples collected during the entire study period. Of the 118 sites surveyed each year in the LARB, the annual number of sites that produced one or more collected hair samples ranged from 90 to 104.

For the TRB, UARB, and LARB, $H_{\mathrm{E}}$ for individual microsatellite loci ranged from 0.16 to 0.78 , 0.30 to 0.77 , and 0.31 to 0.73 ), respectively, across all 23 available loci. On the basis of those values, marker sets consisting of 9, 7, and 8 loci (table 2) were selected for identification of individual bears in the TRB, UARB, and LARB, respectively.

The overall $P I_{\text {sibs }}$ for the TRB was $1.5 \times 10_{-3}$, corresponding to a 1 in 673 chance that a bear shared its multilocus genotype with another bear. Using the Dunn-Sidak method to control the experiment-wise error rate, 3 of 9 microsatellite loci violated Hardy-Weinberg expectations $(\alpha=0.006)$, and 15 of the associations among 36 pairs of loci exhibited linkage disequilibrium $(\alpha=0.001)$. For the UARB, the overall $P I_{\text {sibs }}$ was 3.6E-3, corresponding to a 1 in 274 chance that two bears shared the same multilocus genotype. None of the 7 microsatellite loci violated Hardy-Weinberg expectations ( $\alpha=0.007)$, and 2 of 21 loci pairs exhibited linkage disequilibrium $(\alpha=0.002)$. The overall $P I_{\text {sibs }}$ for the LARB was 3.0E-3 or a 1 in 337 chance that two bears shared the same multilocus genotype. One of 8 loci violated Hardy-Weinberg expectations $(\alpha=0.006)$, and 2 of 28 locus pairs exhibited linkage disequilibrium $(\alpha=0.002)$. Extrapolation of mismatch distribution plots indicated that the expected numbers of $0 M M-$ pairs were $\leq 1$ for the TRB, UARB, and LARB.

From 2006 to 2012, DNA extraction and microsatellite genotyping were attempted for 3,821 hair samples from hair-collection sites surveyed in the TRB. The average annual genotyping success rate was 84 percent (range $=80-89 \%$ ), and the average annual percentage of samples being composed of a mixture of hairs from more than one bear was $<1$. During that same period, 229 hair samples and 18 tissue samples from live-captured bears and mortality recoveries were extracted with success rates of 98 percent and 90 percent, respectively. For the UARB, 1,755 hair samples were submitted for DNA extraction from 2007 to 2012 with an annual average success rate of 79 percent (range $=60-87 \%$ ). Mixed samples were encountered only in 2009 and accounted for 3 percent of those submitted. Twentythree hair samples and 1 tissue sample from live-captured bears and mortality recoveries were extracted with 100-percent success rates. From 2010 to 2012, 1,599 hair samples from hair-collection sites in the LARB were submitted for DNA extraction. An average of 87 percent (range $=81-91 \%$ ) of those samples was successfully genotyped each year, and an average of $<1$ percent was identified as mixtures of hairs from more than one bear. All 25 hair samples collected from live-captured bears and mortality recoveries were successfully genotyped, whereas 6 of 7 (86\%) tissue samples were successfully genotyped.

After collapsing multiple detections into single detections, the CMR dataset for the TRB contained 730 detections of 201 females and 490 detections of 191 males during the entire study period. In the UARB, 62 females were detected 196 times and 47 males 118 times. The LARB dataset contained 175 detections of 91 females and 148 detections of 83 males. In general, the numbers of new individuals entering each dataset each year decreased during the study period, 2006-12 (fig. 7). None of the 13 bears that were present in the TRB as cubs were detected at hair-collection sites. Three of 19 (16\%) bears present as yearlings were detected, and 17 of 30 (57\%) were first detected at age 2. 


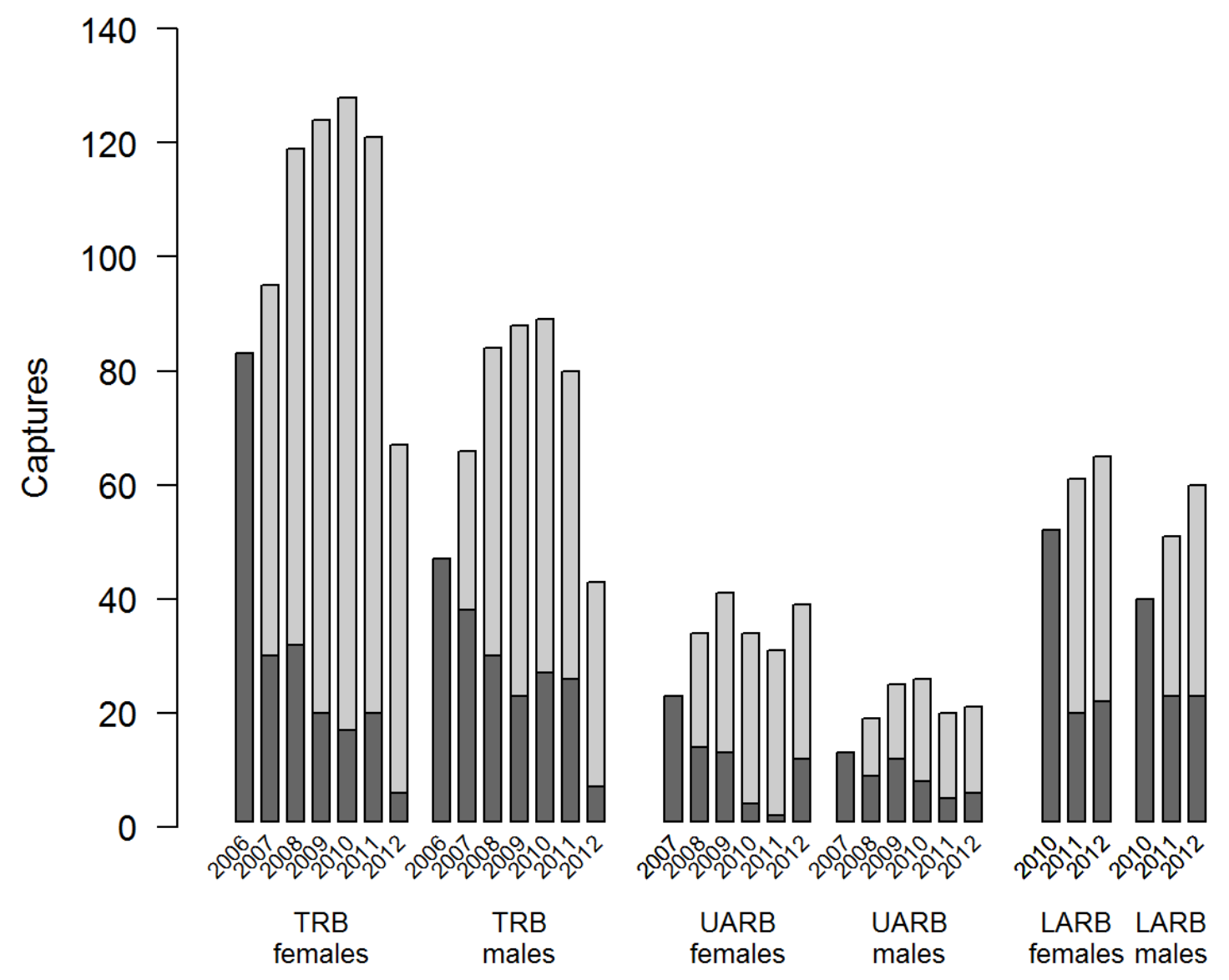

Figure 7. Annual number of DNA-based initial captures (dark gray) and recaptures (light gray) of Louisiana black bears from hair-snare sampling within the Tensas River Basin (TRB), Upper Atchafalaya River Basin (UARB), and Lower Atchafalaya River Basin (LARB) in Louisiana, 2006-12.

When detection heterogeneity was assumed to follow a logistic-normal distribution (Model 1), female abundance estimates for the TRB study area ranged from 141 to 165 from 2006 to 2012 (Model 1, fig. 8). Estimates of annual per capita recruitment $(\gamma)$ ranged from 0.00 to 0.22 , and the annual apparent survival rate $(\phi)$ varied between 0.87 and 0.93 during that period. Density dependence between $N$ and $\gamma$ was negative $\left(\beta_{1}=-0.04\right)$ with 96 percent of the posterior distribution for $\beta_{1}$ being $<0$ (that is, the probability of the relation being negative was 0.96 ). Temporal process variance for $\phi$ on the logit scale was $0.52(95 \% \mathrm{CI}=0.03-2.67)$ and for $\gamma$ on the $\log$ scale was $0.64(95 \% \mathrm{CI}=0.03-6.64)$. Assuming a 2-point finite mixture distribution for detection heterogeneity (Model 2), annual point estimates of female abundances in the TRB ranged from 133 to 158 (fig. 9). Annual estimates of $\gamma$ based on Model 2 ranged from 0.00 to 0.16 , and annual estimates of $\phi$ ranged from 0.87 to 0.89 . Density dependence between $N$ and $\gamma$ was also negative $\left(\beta_{1}=-0.08\right)$ on the basis of Model 2 with 84 percent of the posterior distribution for $\beta_{1}$ being $<0$. 
A

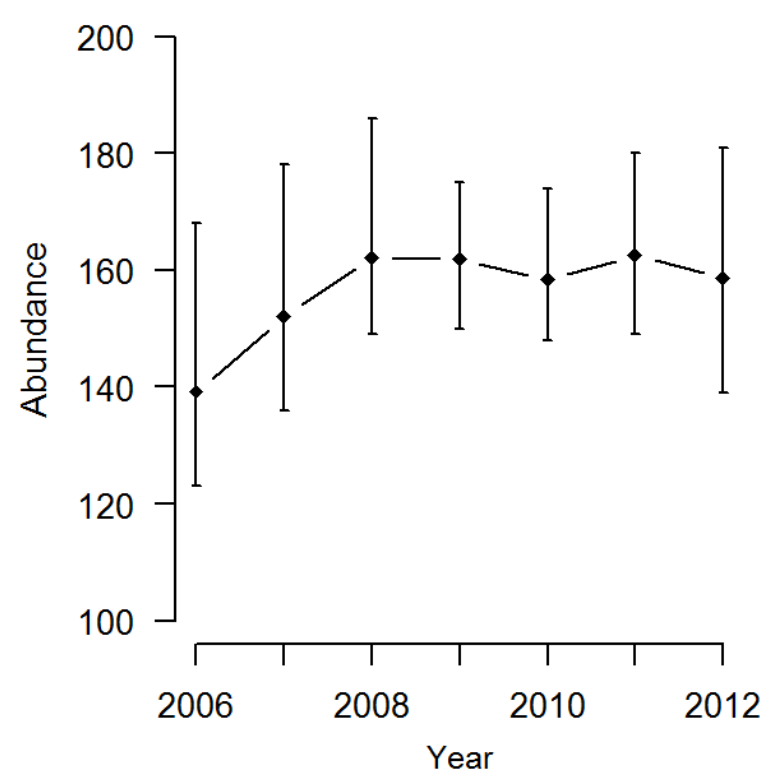

C

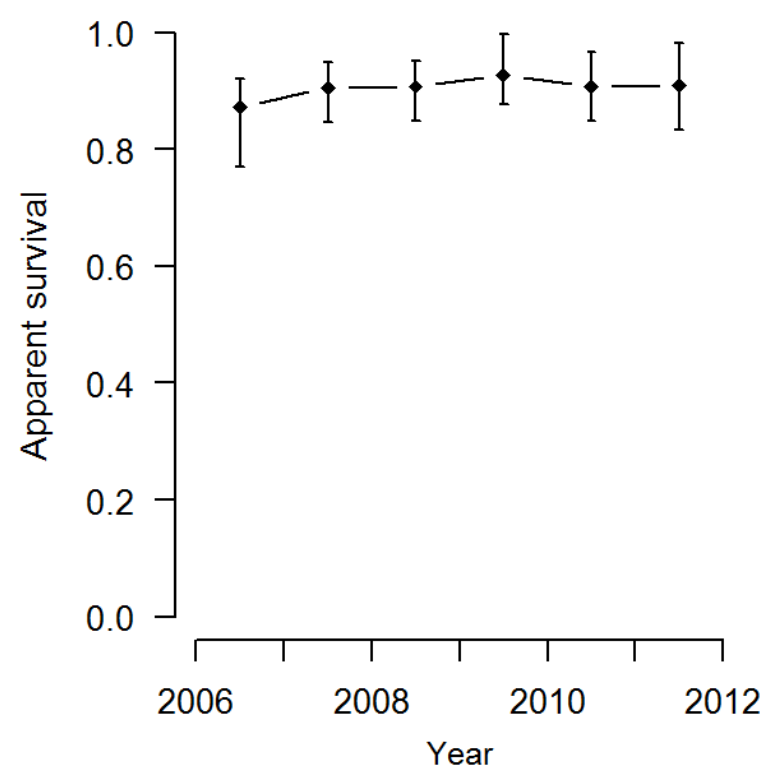

B
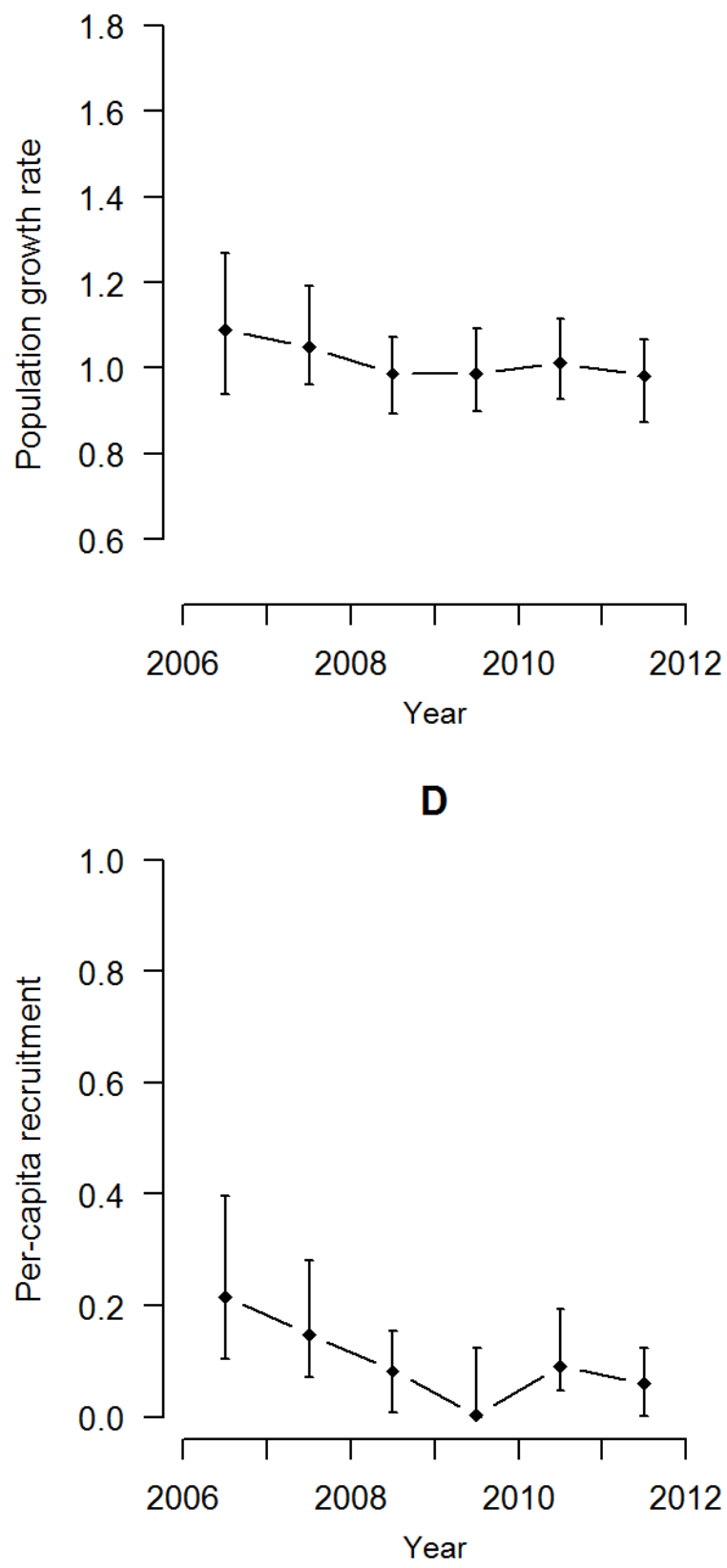

Figure 8. Estimates (diamonds) and 95-percent credible intervals (error bars) of $A$, abundance, $B$, population growth, $C$, apparent survival, and $D$, per-capita recruitment from Model 1 (individual capture heterogeneity modeled with logistic-normal distribution) for female Louisiana black bears within the Tensas River Basin in Louisiana, 200612. 
A
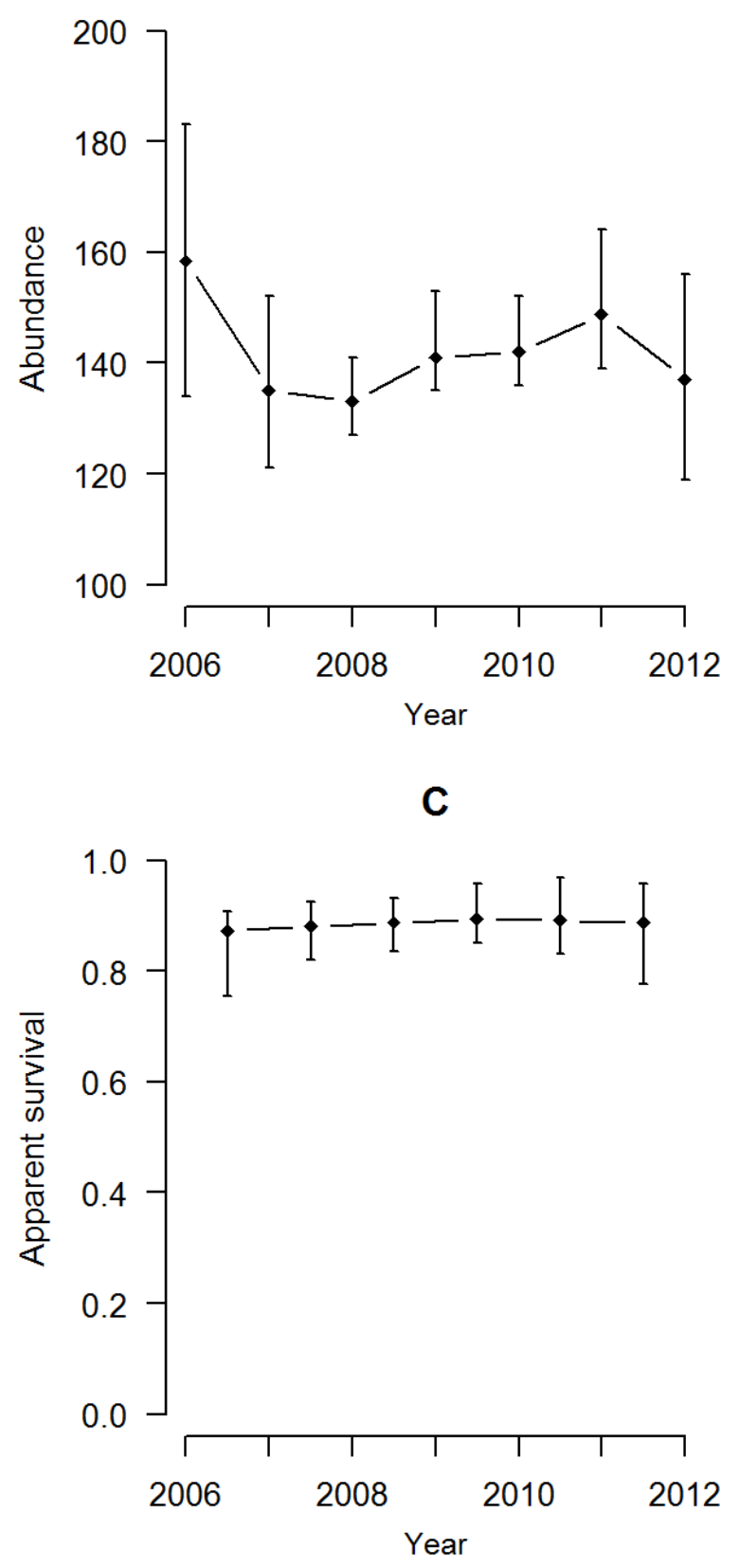

B
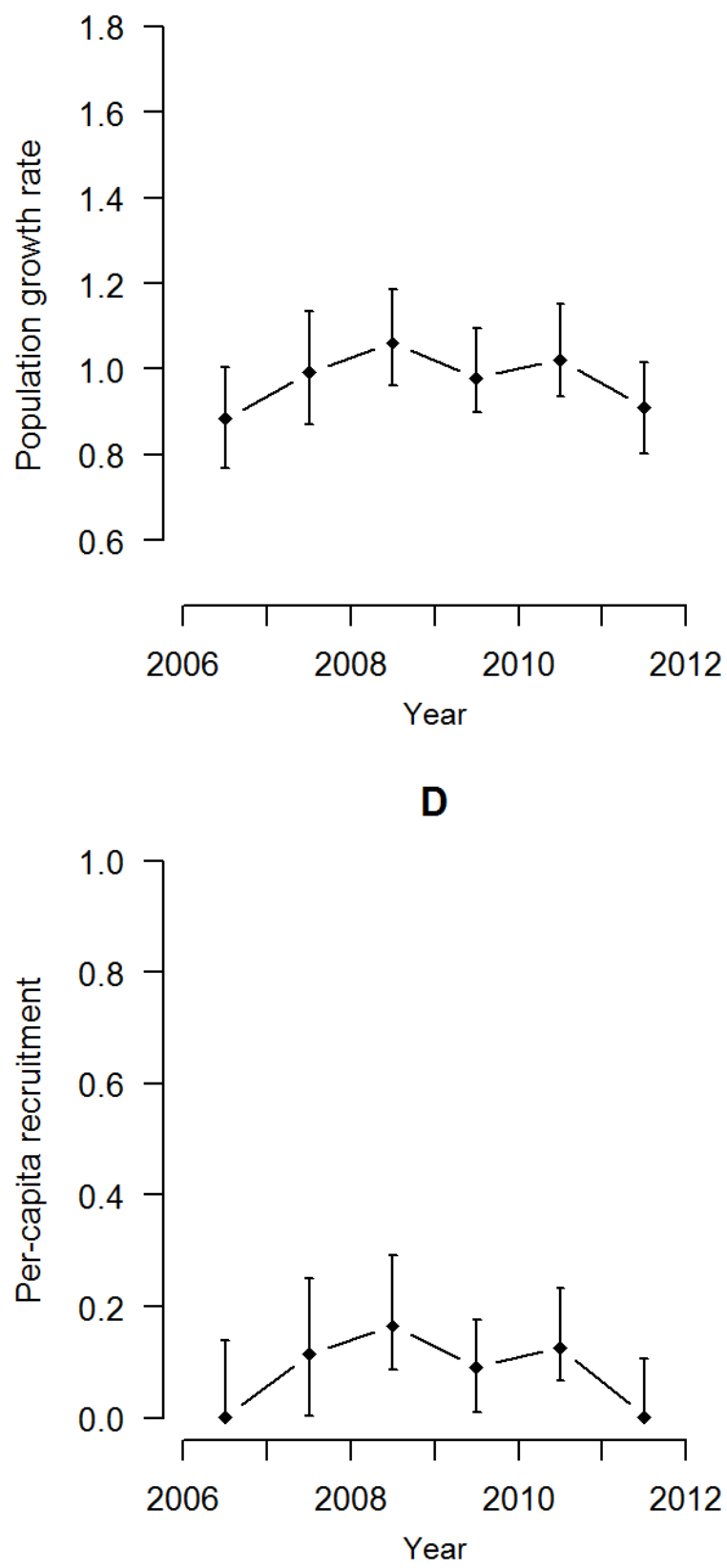

Figure 9. Estimates (diamonds) and 95-percent credible intervals (error bars) of $A$, abundance, $B$, population growth, $C$, apparent survival, and $D$, per-capita recruitment from Model 2 (individual capture heterogeneity modeled with 2-point finite mixture distribution) for female Louisiana black bears within the Tensas River Basin in Louisiana, 2006-12.

In the UARB, $N$ from Model 1 varied between 25 and 44 during 2007-12 (fig. 10). Model 1 estimates of $\gamma$ ranged from 0.00 to 0.41 , and $\phi$ ranged from 0.88 to 0.90 . Similar to the TRB, a negative relation was estimated between $N$ and $\gamma\left(\beta_{1}=-0.09\right)$ with 88 percent of the posterior distribution for $\beta_{1}$ being $<0$. Temporal process variance for $\phi$ on the logit scale was 0.36 (95\% CI $=0.01-2.1$ ) and for $\gamma$ on the log scale was $1.08(95 \% \mathrm{CI}=0.07-8.00)$. When detection heterogeneity was estimated with 
Model 2, annual point estimates of $N$ for the UARB study area ranged from 23 to 41 (fig. 11). Model 2 estimates of $\gamma$ ranged from 0.00 to 0.43 , and estimates of $\phi$ ranged from 0.85 to 0.89 . A negative relation between $N$ and $\gamma\left(\beta_{1}=-0.11\right)$ was again evident with 82 percent of the posterior distribution for $\beta_{1}$ below 0 . Temporal process variance for $\phi$ on the logit scale was $0.69(95 \% \mathrm{CI}=0.03-5.94)$ and for $\gamma$ on the log scale was $1.41(95 \% \mathrm{CI}=0.12-9.02)$.

A

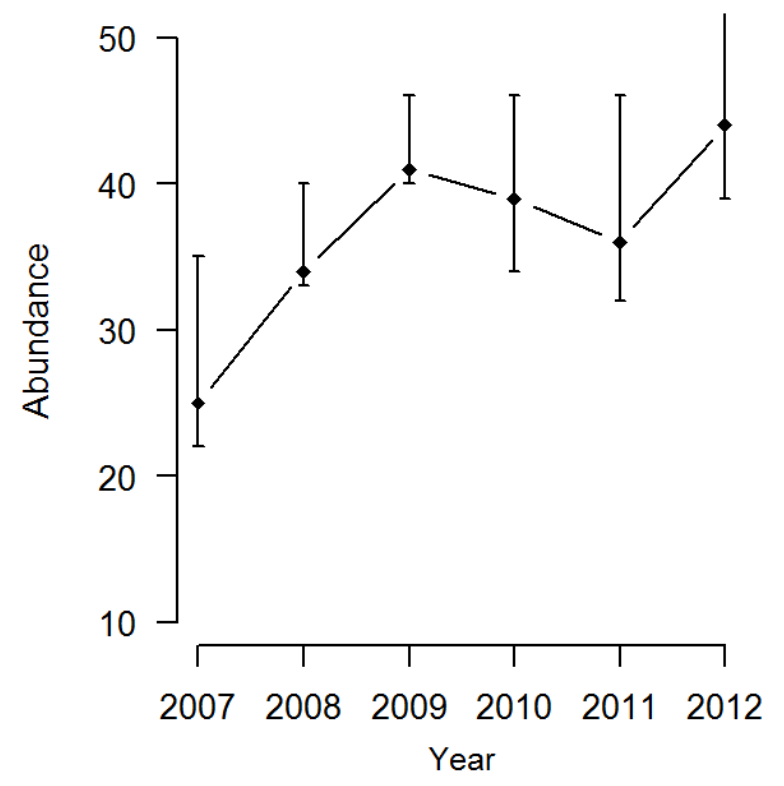

C

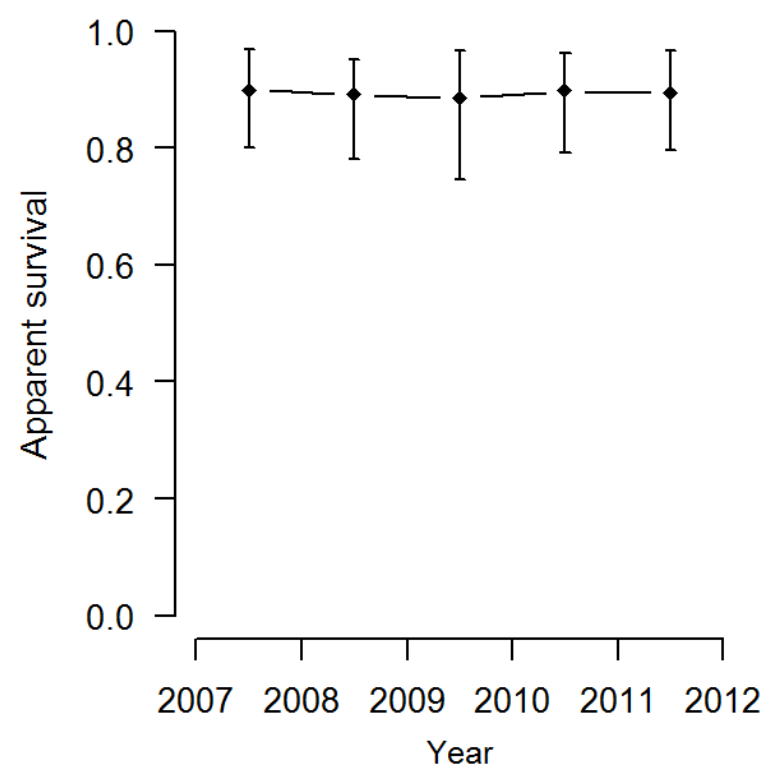

B
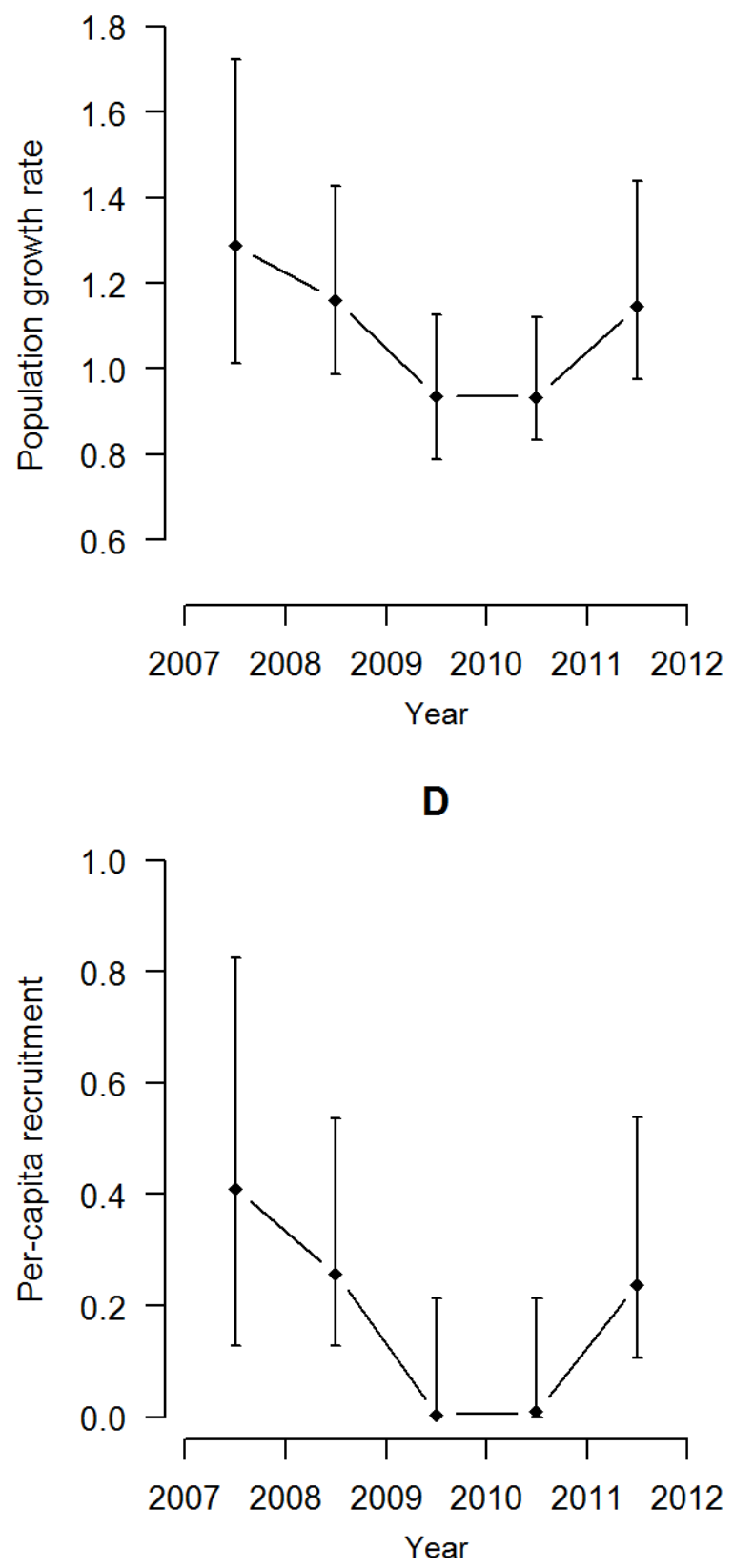

Figure 10. Estimates (diamonds) and 95-percent credible intervals (error bars) of $A$, abundance, $B$, population growth, $C$, apparent survival, and $D$, per-capita recruitment from Model 1 (individual capture heterogeneity modeled with logistic-normal distribution) for female Louisiana black bears within the Upper Atchafalaya River Basin in Louisiana, 2007-12. 
A

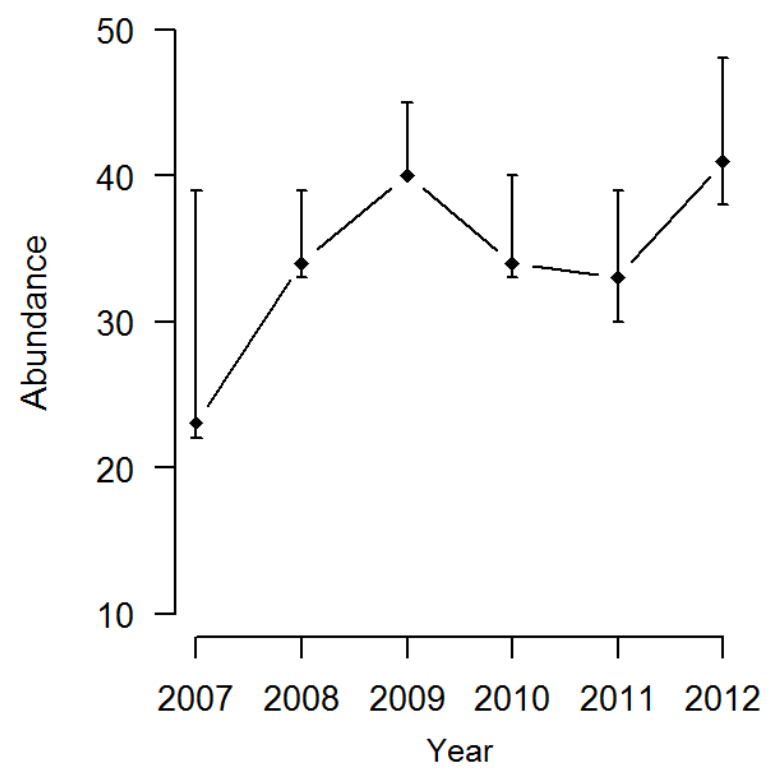

C

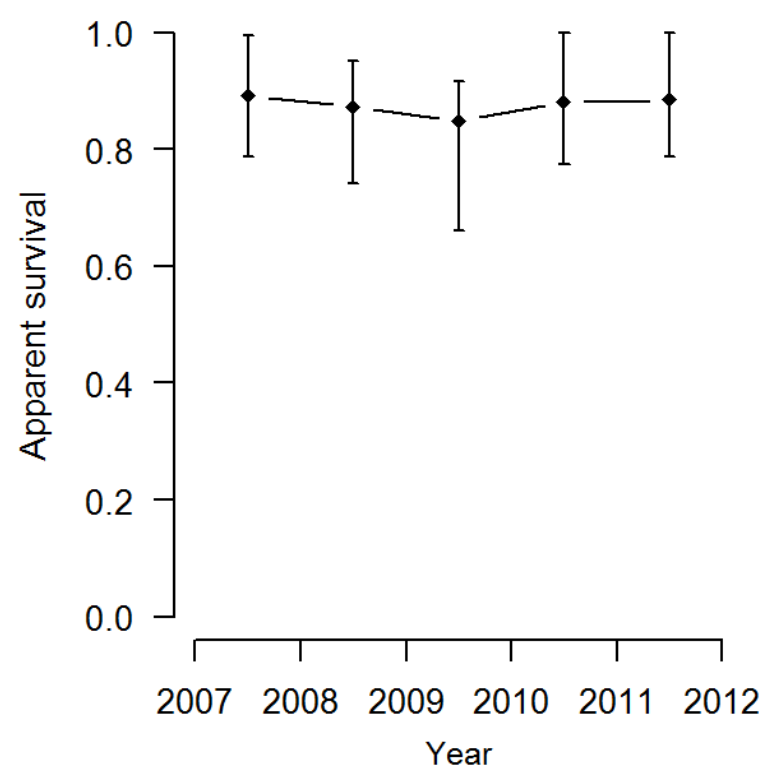

B
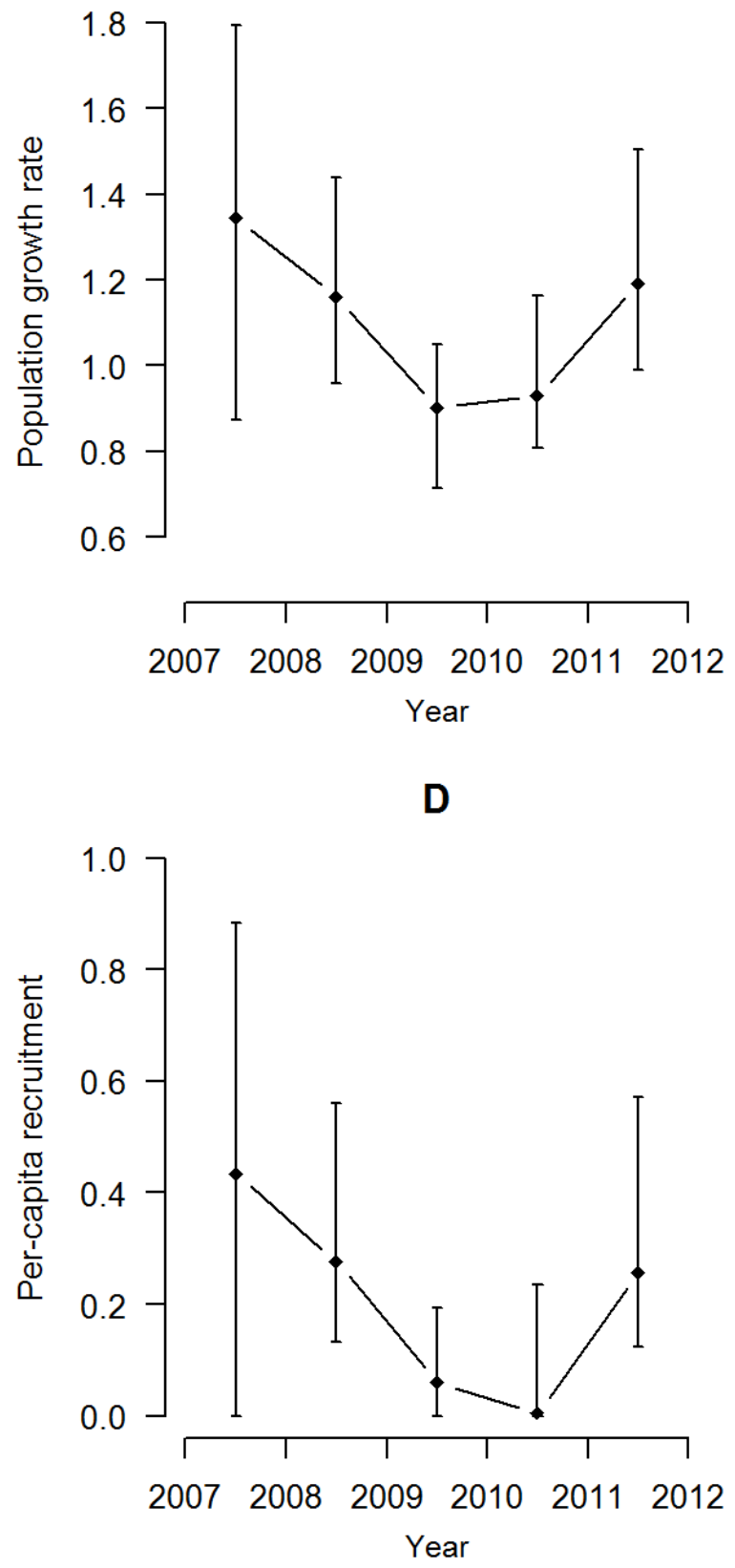

Figure 11. Estimates (diamonds) and 95-percent credible intervals (error bars) of $A$, abundance, $B$, population growth, $C$, apparent survival, and $D$, per-capita recruitment from Model 2 (individual capture heterogeneity modeled with 2-point finite mixture distribution) or female Louisiana black bears within the Upper Atchafalaya River Basin in Louisiana, 2007-12.

Point estimates of female $N$ for the LARB ranged from 78 to 97 from 2010 to 2012, based on Model 1 (fig. 12). Estimates of $\gamma$ were 0.00 (95\% CI $=0-0.03$ ) for $2010-11$ and 0.24 (95\% CI = $0.10-0.50)$ for 2011-12. For those periods, estimates of $\phi$ were $0.81(95 \% \mathrm{CI}=0.68-0.90)$ and 0.85 ( $95 \%$ CI $=0.70-0.94$ ), respectively. On the basis of Model 2, estimates of female abundance for the LARB ranged from 68 to 84 for 2010-12 (fig. 13). Model 2 estimates of $\gamma$ were 0.00 (95\% CI $=0-0.03$ ) 
for 2010- 11 and 0.31 (95\% CI $=0.16-0.51)$ for 2011-12. On the basis of Model 2, estimates of $\phi$ were $0.81(95 \% \mathrm{CI}=0.68-0.90)$ and 0.84 (95\% CI = 0.69-0.97) for 2010- 11 and 2011-12, respectively.

A

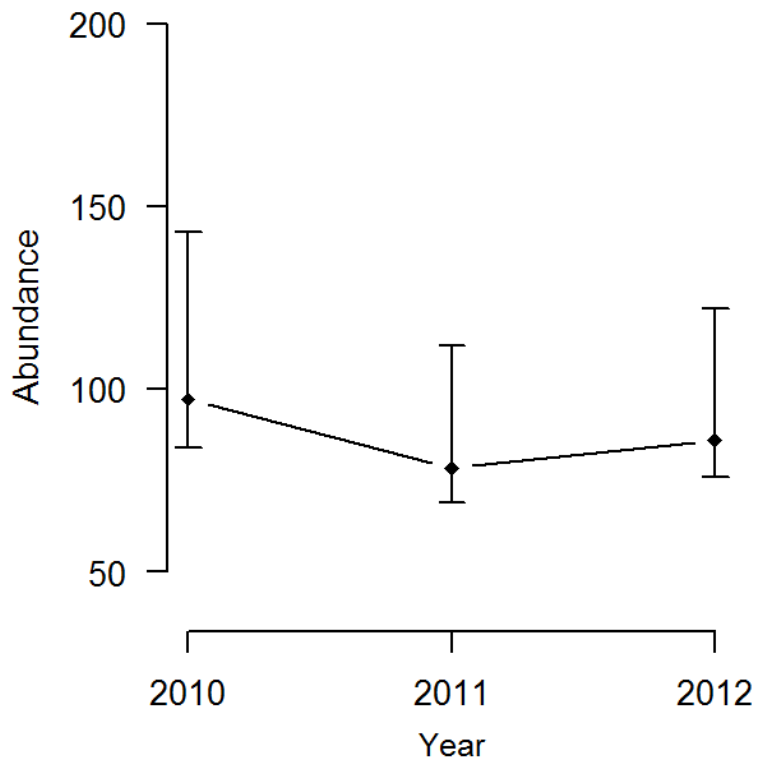

C

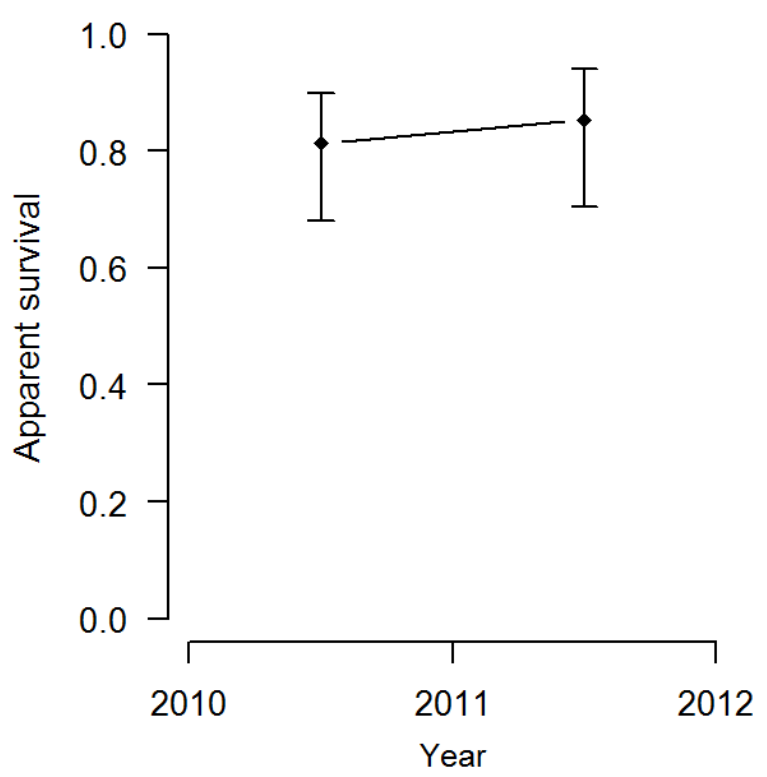

B

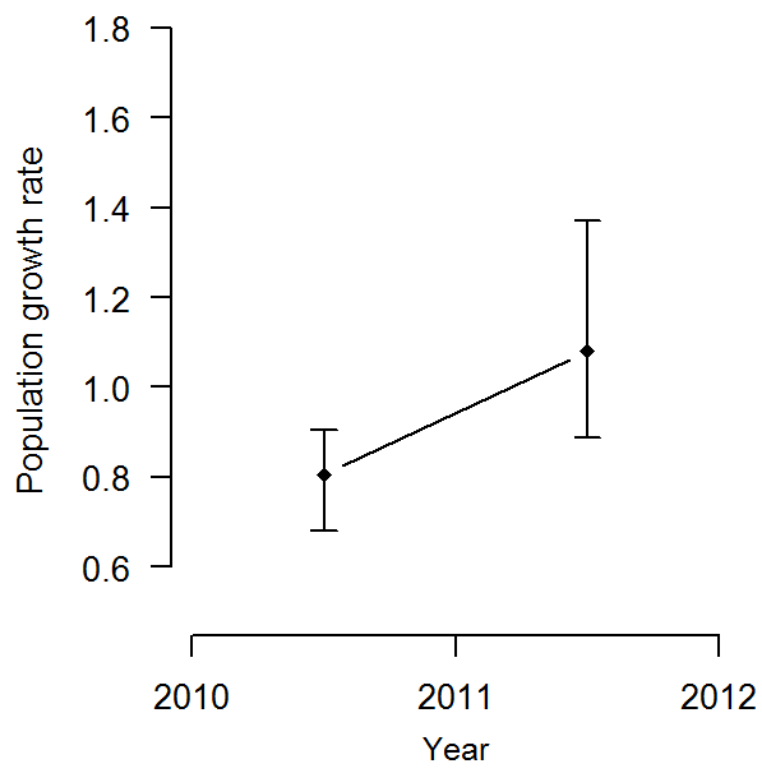

D

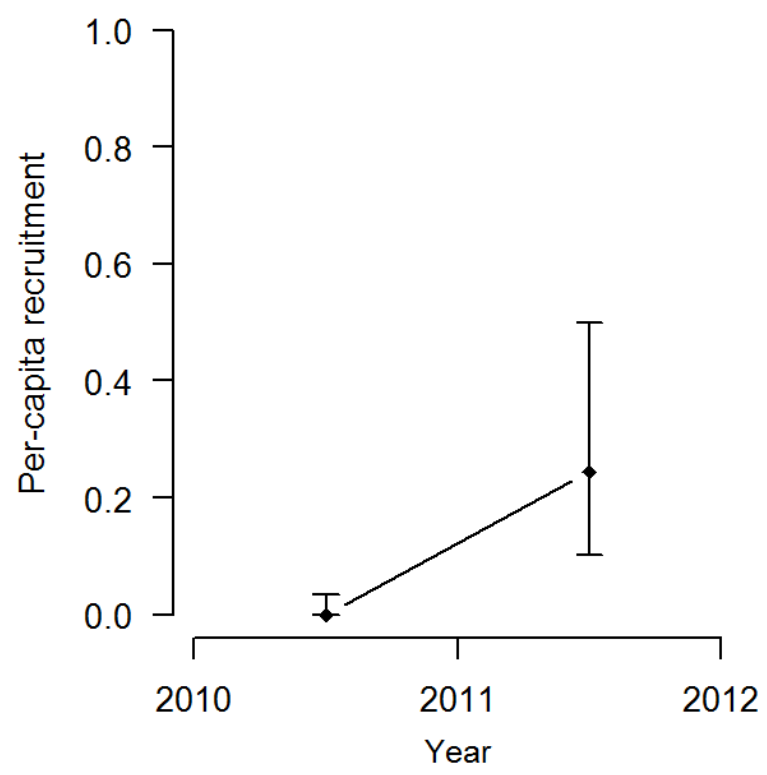

Figure 12. Estimates (diamonds) and 95-percent credible intervals (error bars) of $A$, abundance, $B$, population growth, $C$, apparent survival, and $D$, per-capita recruitment from Model 1 (individual capture heterogeneity modeled with logistic-normal distribution) for female Louisiana black bears within the Lower Atchafalaya River Basin in Louisiana, 2010-12 
A

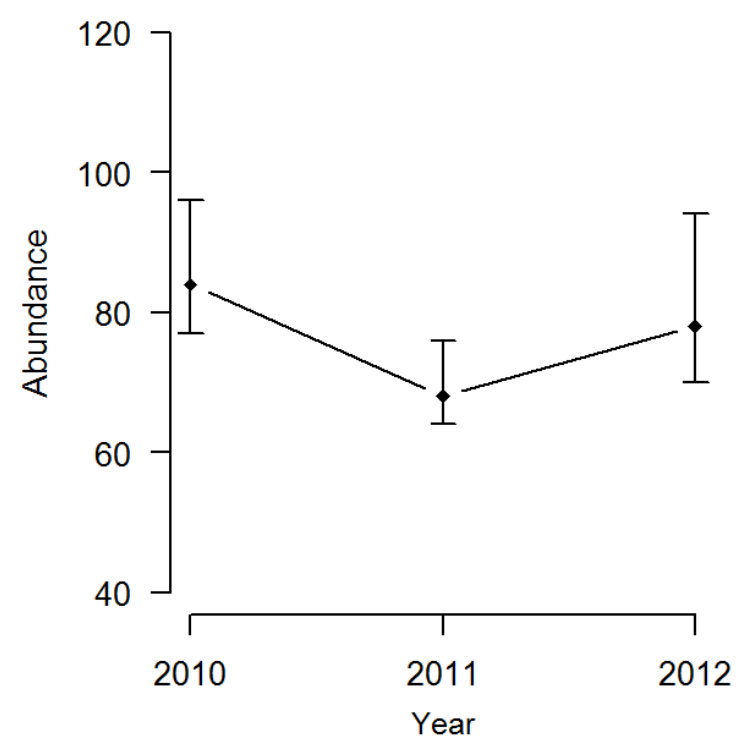

C

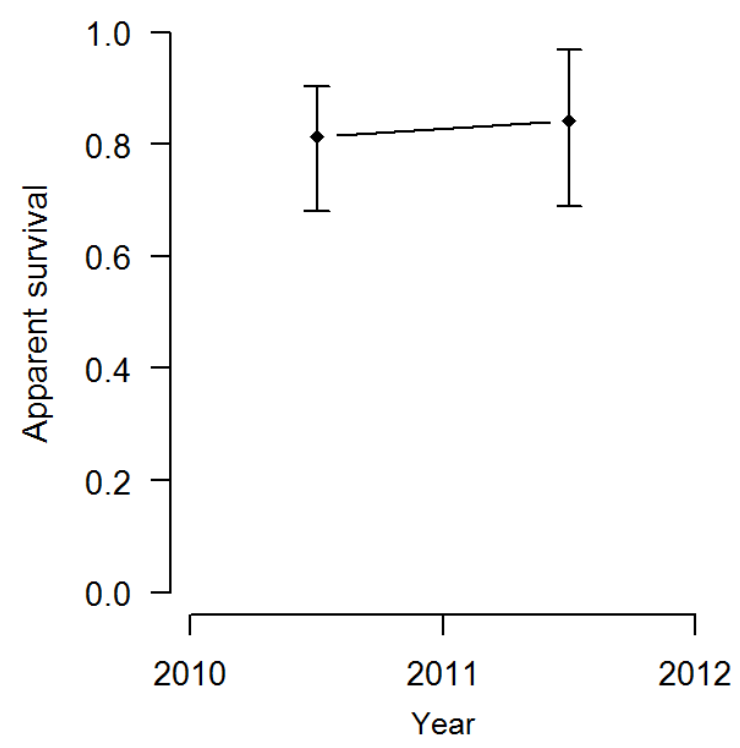

B

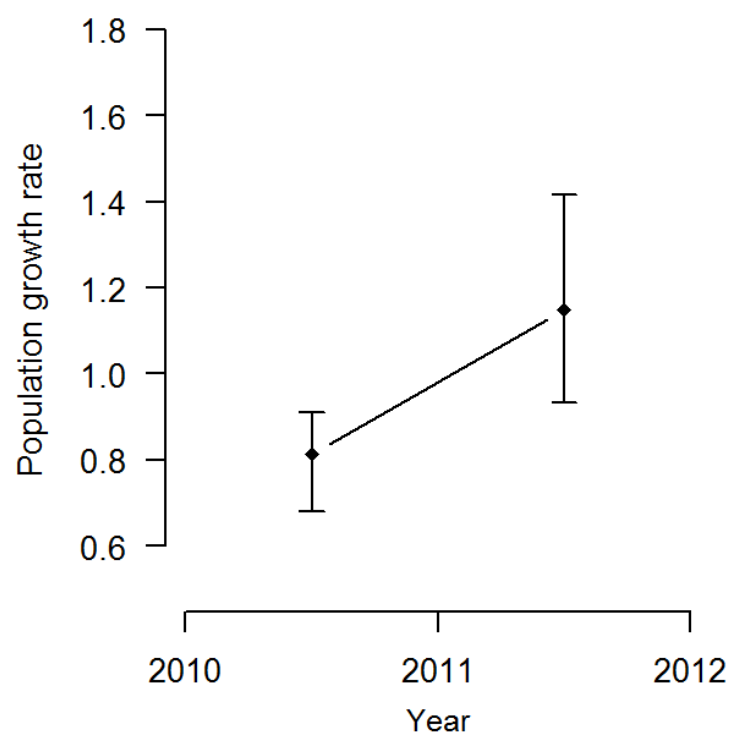

D

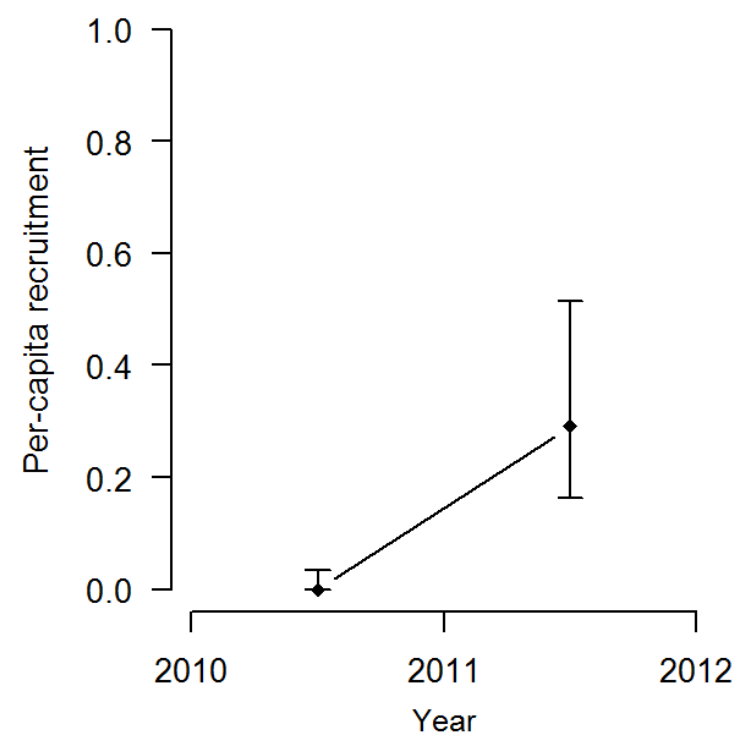

Figure 13. Estimates (diamonds) and 95-percent credible intervals (error bars) of $A$, abundance, $B$, population growth, $C$, apparent survival, and $D$, per-capita recruitment from Model 2 (individual capture heterogeneity modeled with 2-point finite mixture distribution) for female Louisiana black bears within the Lower Atchafalaya River Basin in Louisiana, 2010-12.

Stochastic Population Modeling and Extinction Risk Assessment

Estimated asymptotic growth rates $\left(\lambda_{\text {Asym }}\right)$ for the TRC when unresolved fates were AC and AD were 1.02 and 0.99 , respectively. Estimated $\lambda_{\text {Asym }}$ for the TRB when unresolved fates were AC and AD were 1.04 and 1.02, respectively. The geometric means of realized population growth rate $\left(\bar{\lambda}_{G}\right)$ for TRB were 1.02 (annual range $=0.98-1.09$; fig. 8 ) and 0.97 (annual range $=0.88-1.06$, fig. 9), based on 
Models 1 and 2, respectively. Likewise at UARB, estimates of $\bar{\lambda}_{G}$, based on Models 1 and 2, were 1.08 (annual range $=0.93-1.29$, fig. 10) and 1.09 (annual range $=0.90-1.35$, fig. 11), respectively. For LARB, estimates of $\bar{\lambda}_{G}$ were 0.81 (95\% CI $\left.=0.68-0.91\right)$ for 2010-11 and 1.08 for 2011-12 (95\% CI = 0.89-1.37; fig. 12), based on Model 1. On the basis of Model 2, estimated $\bar{\lambda}_{G}$ for $2010-11$ was 0.81 (95\% CI = 0.68-0.91) and for 2011-12, 1.16 (95\% CI = 0.93-1.41, fig. 13). Because no cubs-of-theyear handled during winter den captures were ever detected at hair-collection sites and were not part of the sampled population for CMR-based demographic rate analyses, our projections for the TRB and UARB pertain to bears $\geq 1$ year of age.

On the basis of vital rate estimates from Model 1 of the CMR analysis, probability of persistence over 100 years for the TRB population was $>0.999,0.975$, and 0.958 for process-only, $50 \%$ CI, and $95 \%$ CI projections, respectively (table 4). Similarly, the probability of persistence was $>0.999,0.982$, 0.958, based on Model 2, for process-only, 50\% CI, and 95\% CI projections, respectively. In general, the probability that the TRB population would decline over the next 100 years (that is, projected $N$ at year 100 less than initial $N$ ) was $>0.70$ for all simulations (table 4 ). The mean percent change in projected abundance for the TRB over 100 years was negative for all simulations.

Table 4. Summary of 10,000 simulated population trajectories over a 100-year period for female Louisiana black bears in the Tensas River Basin (TRB) and Upper Atchafalaya River Basin (UARB), Louisiana. Simulations were based on demographic rates estimated from capture-mark-recapture analyses that modeled capture heterogeneity as individual random effects (Model 1) or finite mixture distributions (Model 2). Simulation models incorporated only temporal-process variation (Process-only), temporal-process variation and parameter uncertainty based on the 50-percent (\%) credible interval (Cl) of Bayesian posterior distributions (All uncertainty-50\% Cl), or temporalprocess variation and parameter uncertainty based on the $95 \%$ credible interval of Bayesian posterior distributions (All uncertainty-95\% Cl).

[LCL, lower credible limit; UCL, upper credible limit]

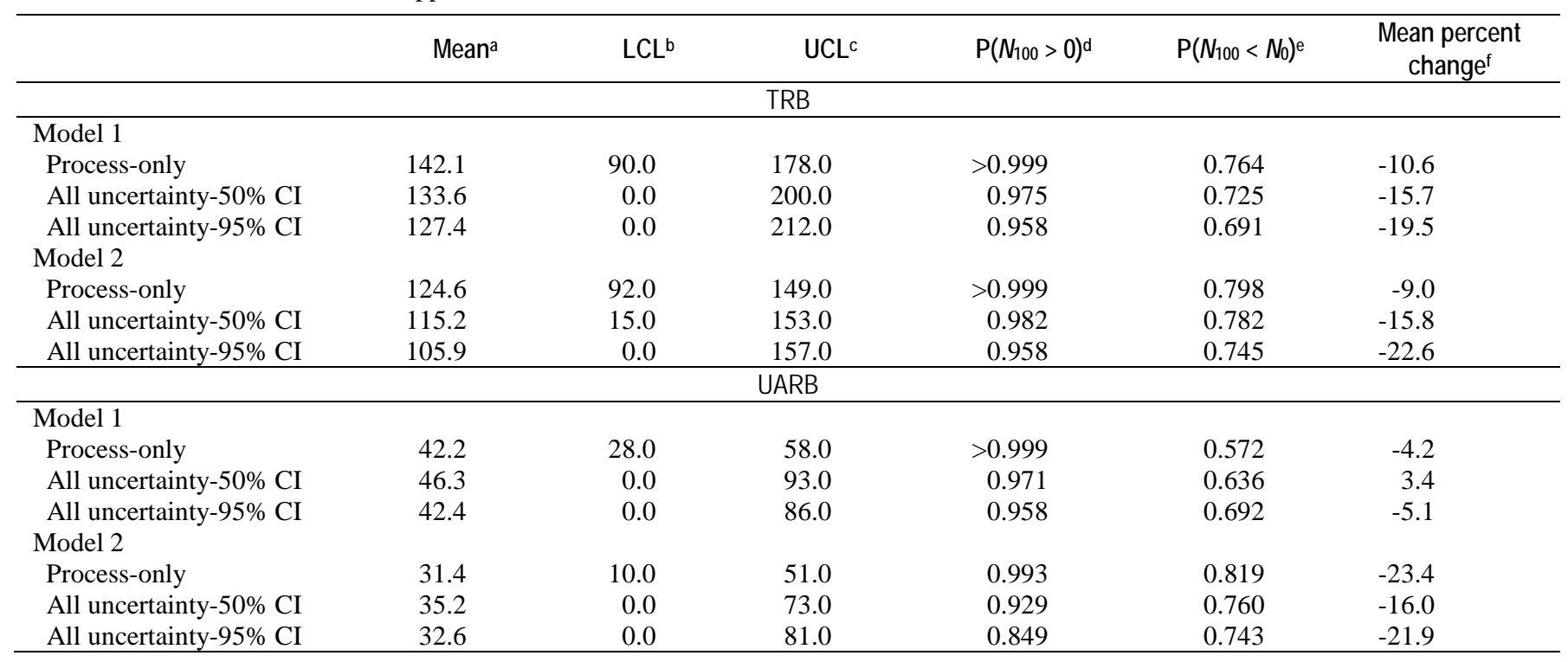

${ }^{\mathrm{a}}$ Mean female abundance after 100 years.

b2.5 percentile of distribution of abundances after 100 years.

${ }^{c} 97.5$ percentile of distribution of abundances after 100 years.

${ }^{\mathrm{d}}$ Probability of population persistence after 100 years.

${ }^{\text {e}}$ Probability of female abundance after 100 years less than starting female abundance.

${ }^{\mathrm{f}}$ Percent change in female abundance over 100 years averaged over 10,000 simulated population trajectories. 
For the UARB, probabilities of persistence based on Model 1 were $>0.999,0.971$, and 0.958 for process-only, 50\% CI, and 95\% CI projections, respectively, and based on Model 2, 0.993, 0.929. and 0.849 (table 4). Similar to the TRB, the mean percent change in projected abundance over 100 years was negative for all simulation scenarios except for the scenario based on Model 1 vital rate estimates and incorporating uncertainty based on the 50-percent CI projection model. However, further inspection of abundances after 100 years for those simulations revealed several large outlier values (that is, $N_{100}>1,000$ ) that caused right skewness and an inflated arithmetic mean. The probability that $N$ will decline ranged from 0.578 to 0.819 , depending on projection model (table 4). Projected $N$ at year 100 for the TRB and UARB was consistently greater for simulations based on vital rates from CMR Model 1 than for simulations based on Model 2 estimates (table 4).

Using the telemetry and reproductive data from the TRC, probabilities of persistence were about 3 times greater for AC projections than for AD projections when temporal process-only variance of vital rates was incorporated, regardless of assumed strength of density dependence or the CMR model used to derive carrying capacity (tables 5 and 6). When all uncertainty of vital rate estimates was incorporated, probabilities of persistence were about 1.6-1.7 times greater for AC than for AD (tables 5 and 6). Persistence probabilities were $\geq 0.95$ only for projections based on AC assumptions and incorporating process-only variation. Moreover, values of $\bar{N}_{100}$ from AC projections were more variable, which reflects uncertainty in vital rate values used in those projections. Probabilities of persistence for population projections based on equilibrium abundance estimates derived from the UARB were, in most cases, nearly identical to those based on abundance estimates derived from the TRB. However, values of $\bar{N}_{100}$ were consistently lower for all UARB-based scenarios because of the lower equilibrium abundance used. Differences in the assumed strength of the density-dependence relation used in projections had only a minor influence on all persistence probabilities (tables 5 and 6). Assuming dynamics of the TRB, TRC, and UARB populations were independent and using the most pessimistic population-specific persistence probabilities (that is, $0.958,0.295$, and 0.849 , respectively), the overall probability of persistence for bears in the population system was 0.996 . 
Table 5. Summary of 10,000 simulated population trajectories over a 100-year period for female Louisiana black bears in the Three Rivers Complex (TRC), Louisiana. Simulations were based on adult survival rates estimated from radio-telemetry data and reproductive rates estimated from den visit data, incorporated only process variation (Process-only) or process variation and parameter uncertainty (All-uncertainty), and included different strengths of density dependence $(\theta=0.1$ or 0.5$)$ using the Michaelis-Menten function for enzyme kinetics. Simulations were conducted separately for estimates of adult survival rates that treated unresolved radio losses as censored (Assumed censored) and estimates that treated those losses as mortalities (Assumed dead). Carrying capacity (CC) based on density estimates derived from capture-mark-recapture analyses modeling capture heterogeneity with random effects (Model 1).

[LCL, lower credible limit; UCL, upper credible limit]

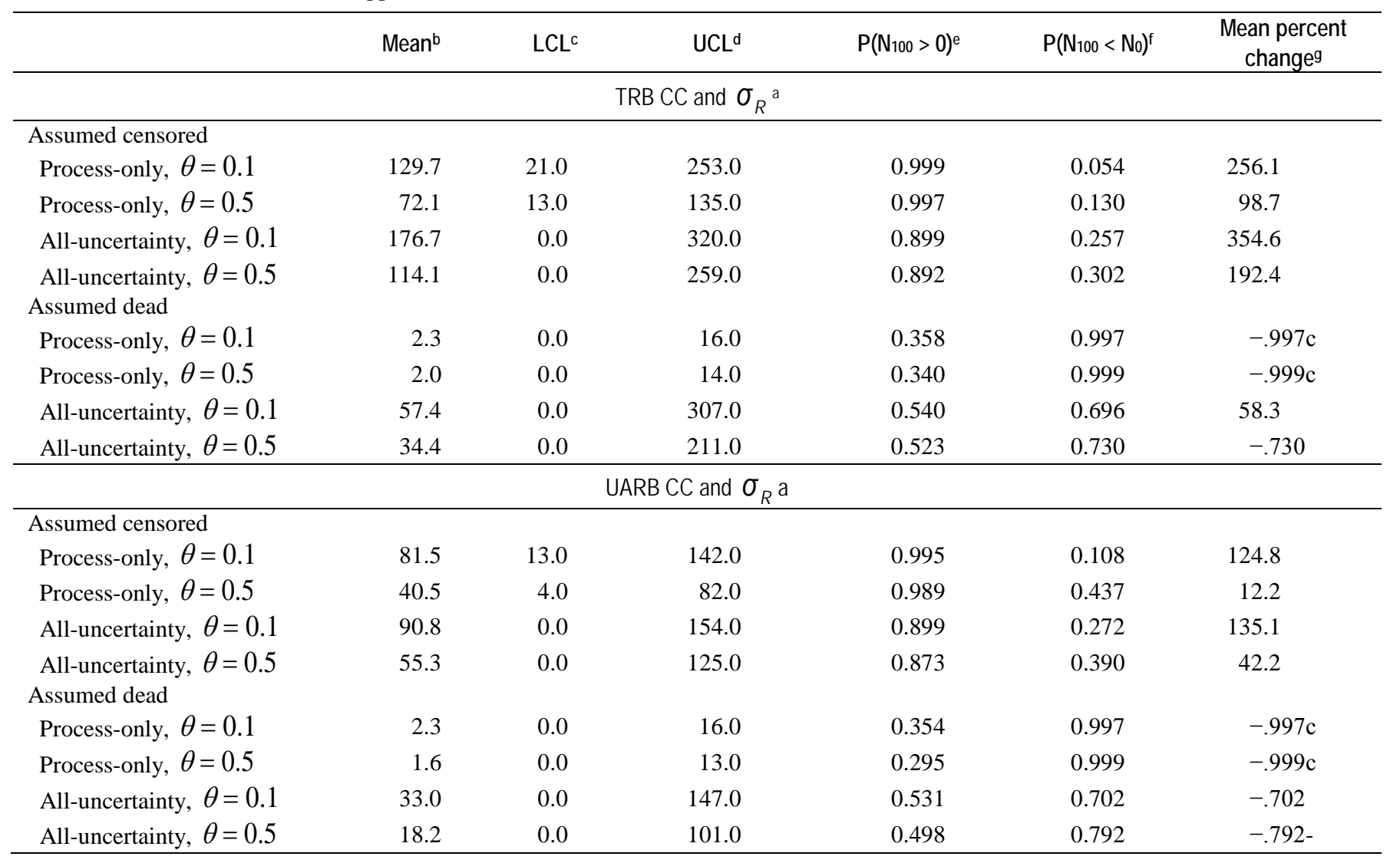

${ }^{\mathrm{a} C}$ Carrying capacity $(C C)$ and process variance for reproduction $\left(\sigma_{R}\right)$ based on Tensas River Basin (TRB) or Upper Atchafalaya River

Basin (UARB) in Louisiana.

${ }^{\mathrm{b}}$ Mean female abundance after 100 years.

${ }^{\mathrm{c}} 2.5$ percentile of distribution of abundances after 100 years.

${ }^{\mathrm{d}} 97.5$ percentile of distribution of abundances after 100 years.

${ }^{\text {e}}$ Probability of persistence after 100 years.

${ }^{\mathrm{f}}$ Probability of female abundance after 100 years less than starting female abundance.

${ }^{g}$ Percent change in abundance over 100 years $\left(\%\right.$ change $\left.=100 \times\left(N_{100}-N_{0}\right) / N_{100}\right)$ averaged over 10,000 simulated population trajectories. 
Table 6. Summary of 10,000 simulated population trajectories over a 100-year period for female Louisiana black bears in the Three Rivers Complex (TRC), Louisiana, USA. Simulations were based on adult survival rates estimated from radio-telemetry data and reproductive rates estimated from den visit data, incorporated only process variation (Process-only) or process variation and parameter uncertainty (All-uncertainty), and included different strengths of density dependence $(\theta=0.1$ or 0.5$)$ using the Michaelis-Menten function for enzyme kinetics. Simulations were conducted separately for estimates of adult survival rates that treated unresolved radio losses as censored (Assumed censored) and estimates that treated those losses as mortalities (Assumed dead). Carrying capacity $(C C)$ based on density estimates derived from capture-mark-recapture analyses modeling capture heterogeneity with 2-point finite mixture distribution (Model 2).

[LCL, lower credible limit; UCL, upper credible limit]

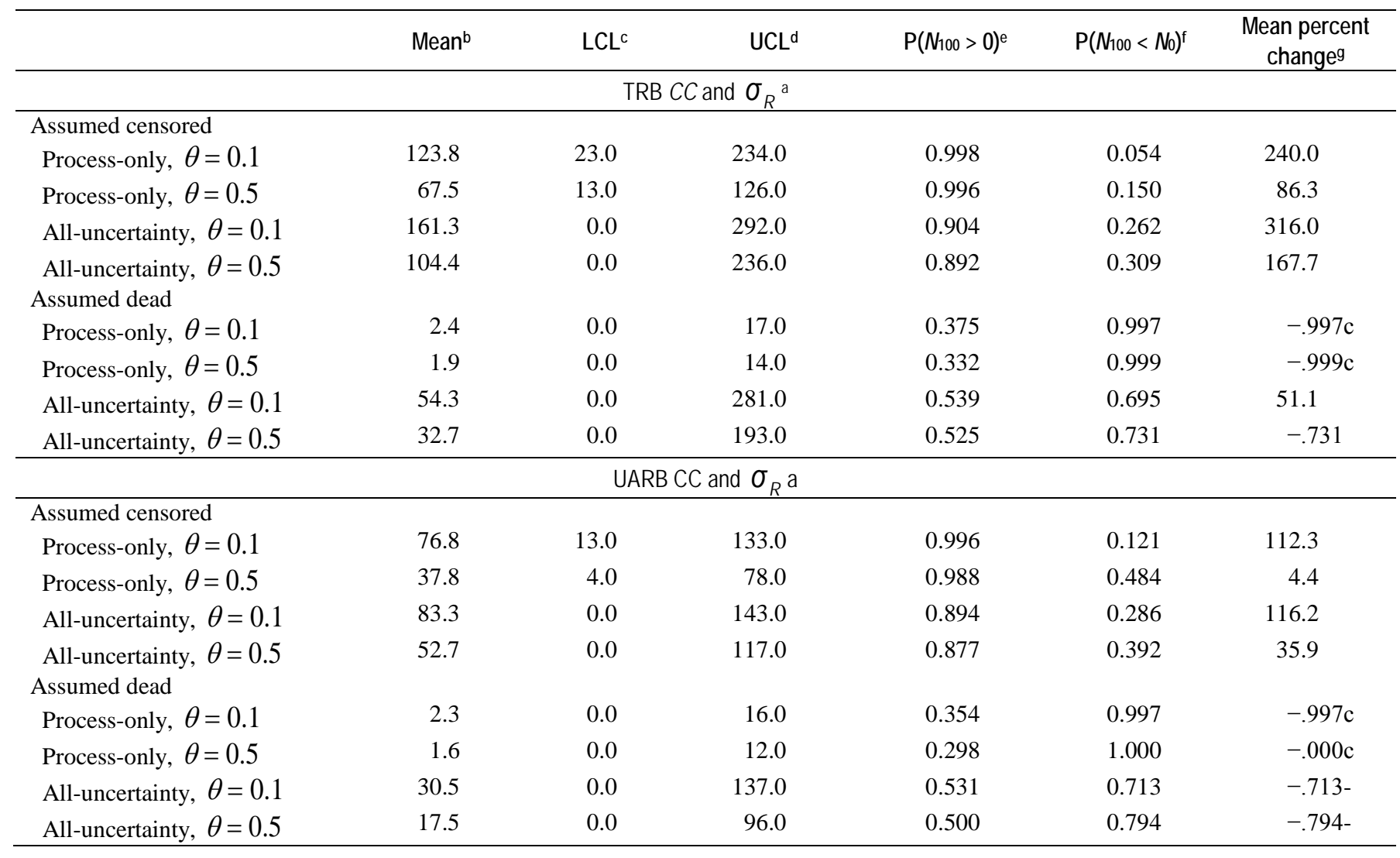

${ }^{\text {a }}$ Carrying capacity $(C C)$ and process variance for reproduction $\left(\sigma_{R}\right)$ based on Tensas River Basin (TRB) or Upper Atchafalaya River

Basin (UARB) in Louisiana, USA.

${ }^{\mathrm{b}}$ Mean female abundance after 100 years.

${ }^{\mathrm{c}} 2.5$ percentile of distribution of abundances after 100 years.

${ }^{\mathrm{d}} 97.5$ percentile of distribution of abundances after 100 years.

${ }^{\mathrm{e}}$ Probability of population persistence after 100 years.

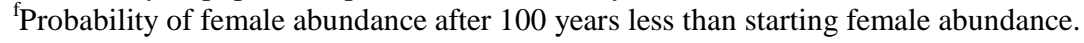

${ }^{\mathrm{g}}$ Percent change in abundance over 100 years $\left(\%\right.$ change $\left.=100 \times\left(N_{100}-N_{0}\right) / N_{100}\right)$ averaged over 10,000 simulated population trajectories. 


\section{Connectivity Analysis}

\section{Population Structure Analysis from Genetic Data}

Clustering results from the factorial correspondence analysis indicated varying levels of genetic structure among different pairs of study populations. When all populations were included in the analysis, four distinct clusters were identifiable corresponding to the MINN, UARB, and LARB populations along with a composite population (COMP) composed of all bears from the WRB and TRB, most MISS bears, and about one-half of the TRC bears (fig. 14). Genetic structure appeared to be greatest between COMP and LARB, COMP and UARB, and UARB and LARB pairs, and appeared to be lowest between MINN and UARB. Additionally, 7 bears from MISS had genetic structure between the LARB and MINN clusters, and 50 percent of the TRC bears and 3 MISS bears had genetic structures between the TRB and UARB, indicating mixed ancestry or potentially genetically distinct groups. FCA results restricted to bears from the MINN, TRB, UARB, and LARB populations revealed four distinct clusters corresponding to the true population of origin for those individuals (fig. 15). However, the MINN and UARB clusters slightly overlapped along axis 1 of the FCA indicating considerably less genetic structure between those two populations than for other population pairs. When only bears from the TRB, TRC, and UARB were considered, the TRB and UARB populations appeared as substantially distinct genetic groups, whereas bears in the TRC were divided between individuals clustering with the TRB and those whose genotypes were clustered mid-way between the TRB and UARB (fig. 16). Results of analysis based only on bears from the WRB and TRB revealed greater genetic structure between those populations than for pairings between MINN and WRB, TRB, TRC, LARB, or MISS but less structure than for the MINN-UARB pair. However, sufficient genetic structure appeared to exist between the WRB and TRB, such that recent migrants could be identified (for example, bears sampled in the TRB clustering with WRB; fig. 17). Taken together, results from the all population and the WRB-TRB clustering analyses indicate at least five genetically distinct populations are represented in the genetic data. 


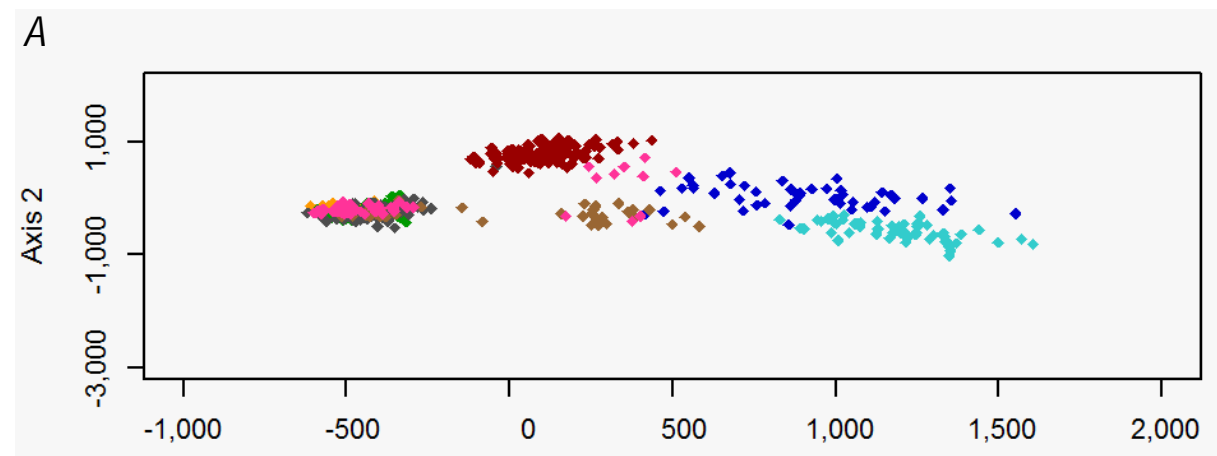

$B$

Axis 1

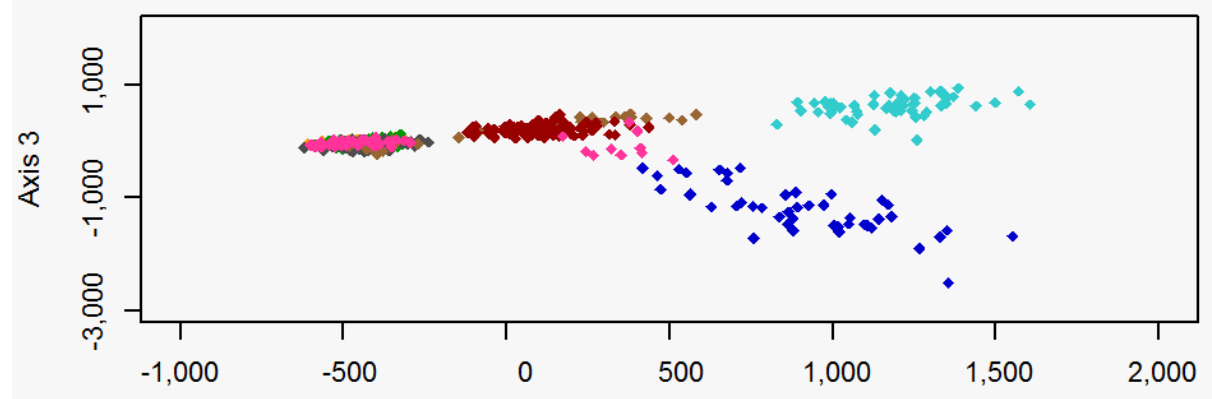

C

Axis 1

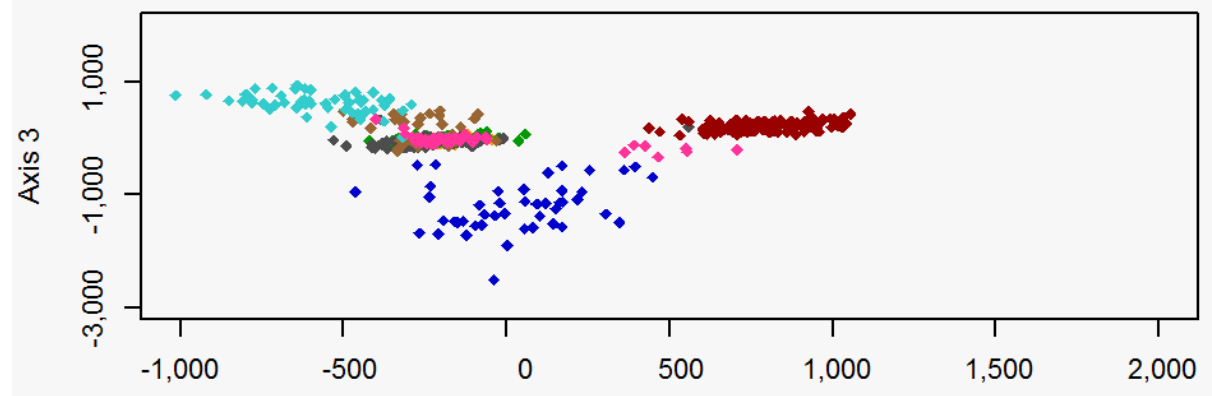

Axis 2

Figure 14. Factorial correspondence analysis results for black bears in Minnesota (blue), Mississippi (pink), the White River Basin (orange) in Arkansas, and the Tensas River Basin north of Interstate 20 (green), Tensas River Basin south of Interstate 20 (gray), Three Rivers Complex (brown), Upper Atchafalaya River Basin (light blue), and Lower Atchafalaya River Basin (red) in Louisiana: A, Axis 1 versus Axis 2; $B$, Axis 1 versus Axis 3; $C$, Axis 2 versus Axis 3. 
A

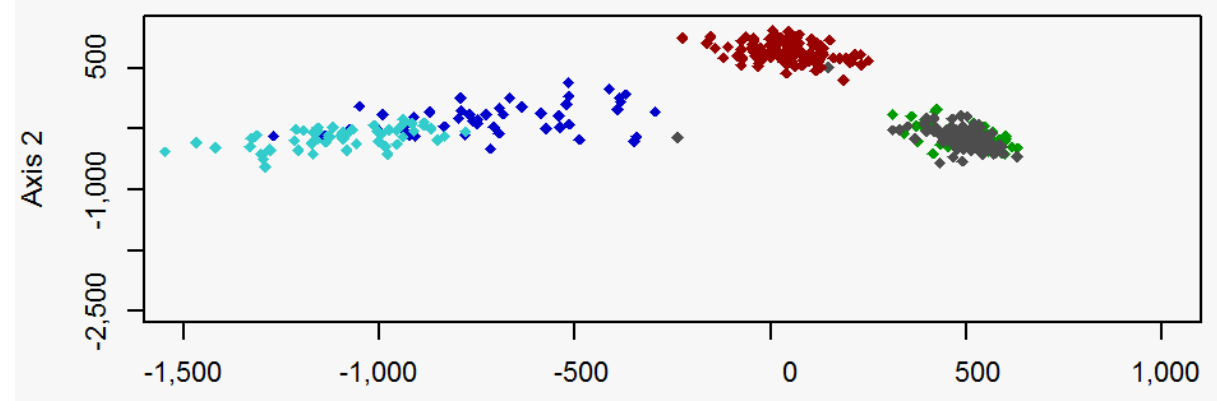

Axis 1

$B$

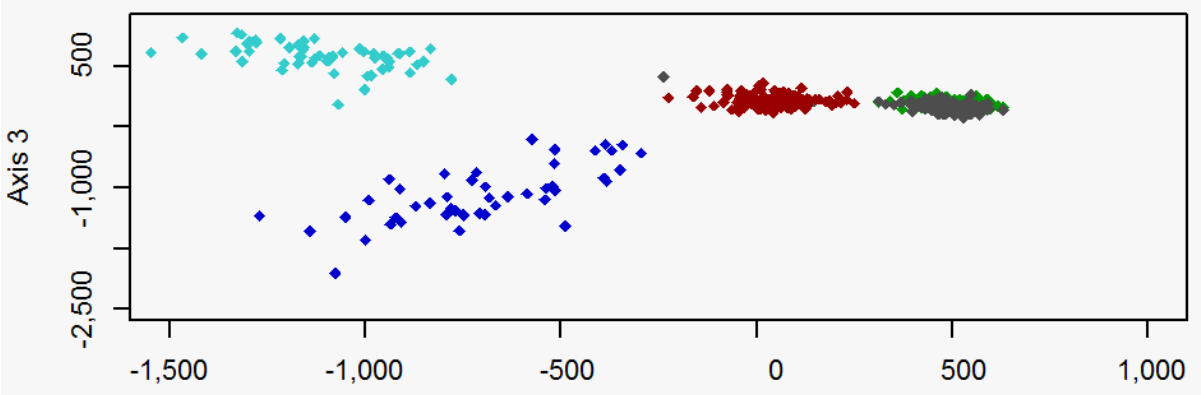

C

Axis 1

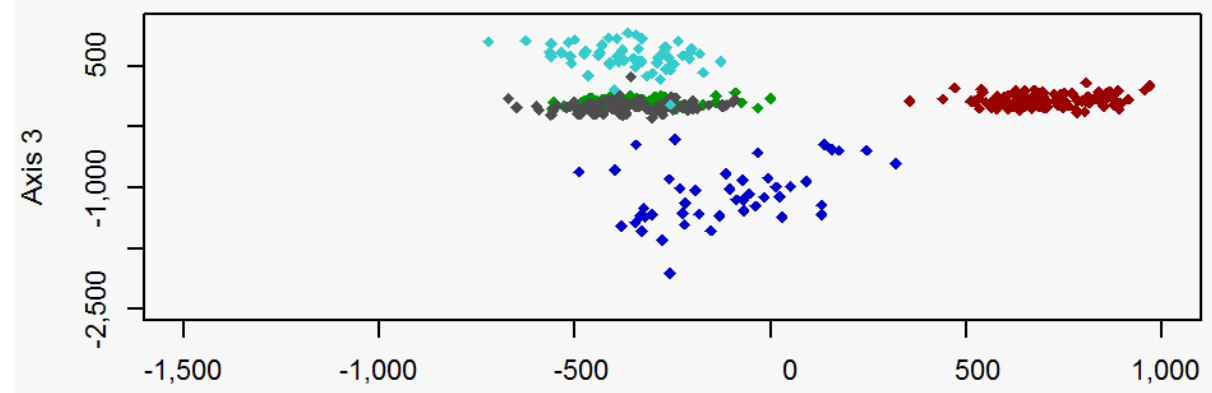

Axis 2

Figure 15. Factorial correspondence analysis results for black bears in Minnesota (dark blue) and the Tensas River Basin north of Interstate 20 (green), Tensas River Basin south of Interstate 20 (gray), Upper Atchafalaya River Basin (light blue), and Lower Atchafalaya River Basin (red) in Louisiana: A, Axis 1 versus Axis 2; $B$, Axis 1 versus Axis 3; $C$, Axis 2 versus Axis 3 
$A$

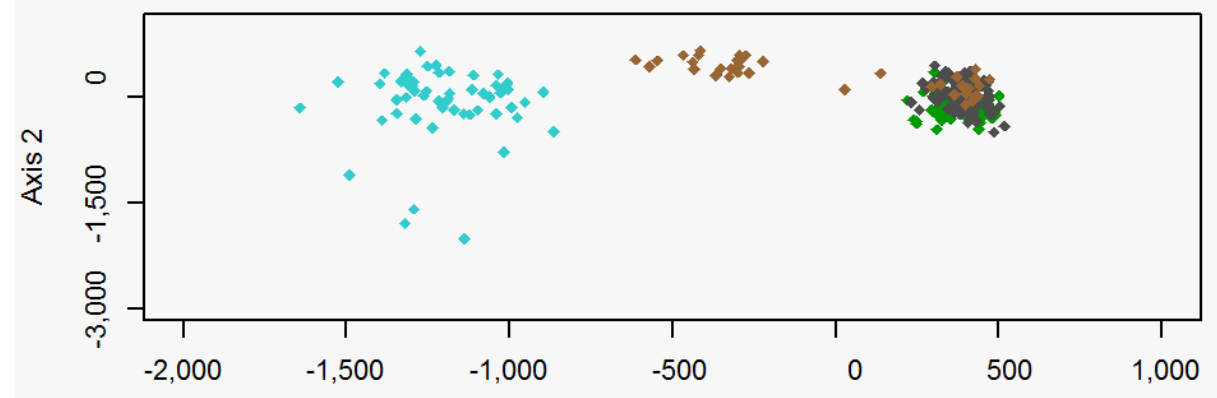

$B$

Axis 1

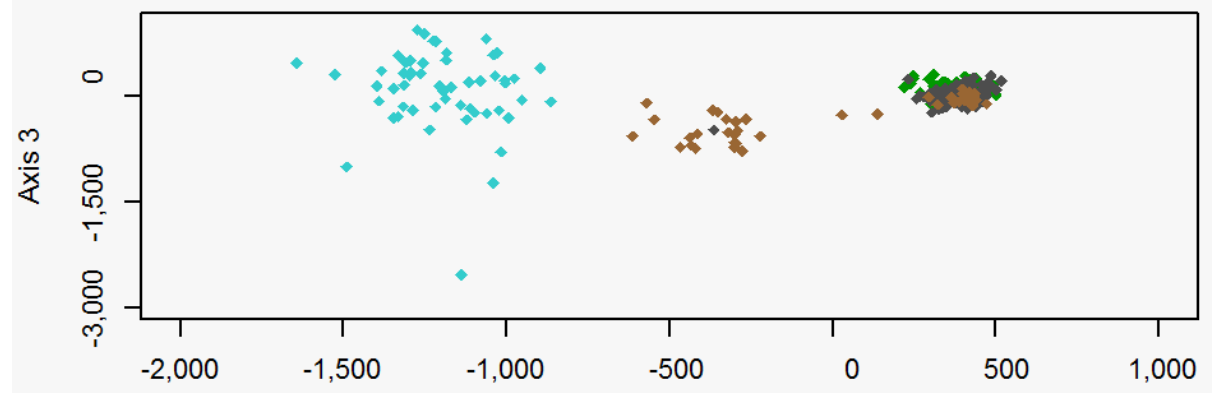

C

Axis 1

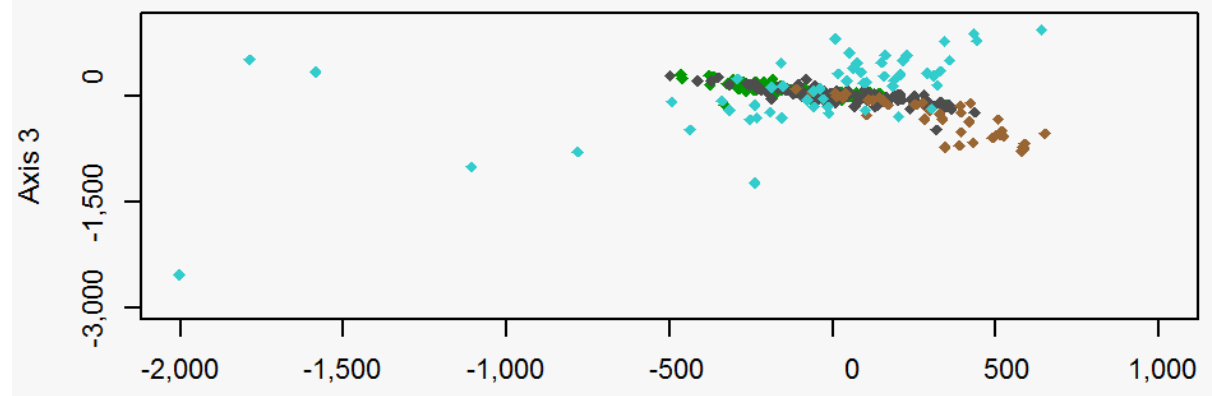

Axis 2

Figure 16. Factorial correspondence analysis results for black bears within the Tensas River Basin north of Interstate 20 (green), Tensas River Basin south of Interstate 20 (gray), Three Rivers Complex (brown), and Upper Atchafalaya River Basin (light blue) in Louisiana: A, Axis 1 versus Axis 2; B, Axis 1 versus Axis 3; $C$, Axis 2 versus Axis 3. 


\section{A}

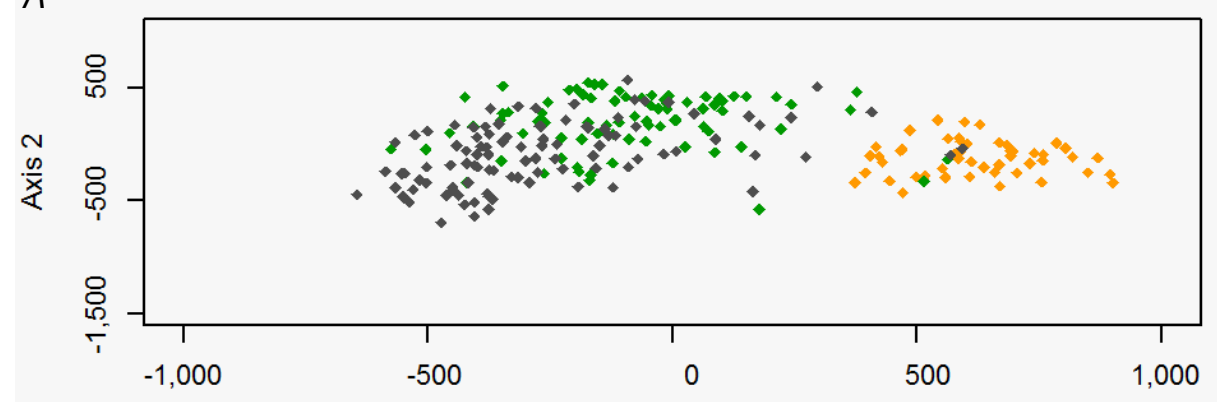

Axis 1

$B$

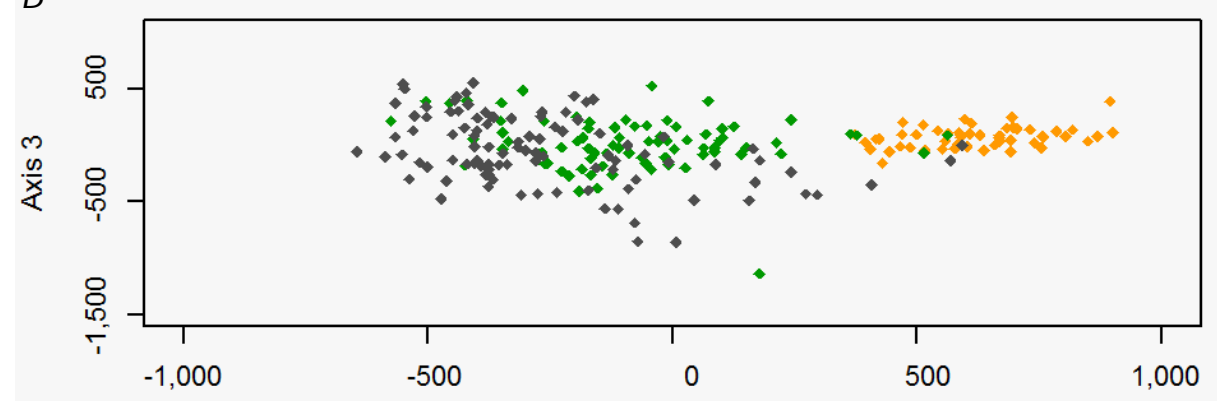

Axis 1

$C$

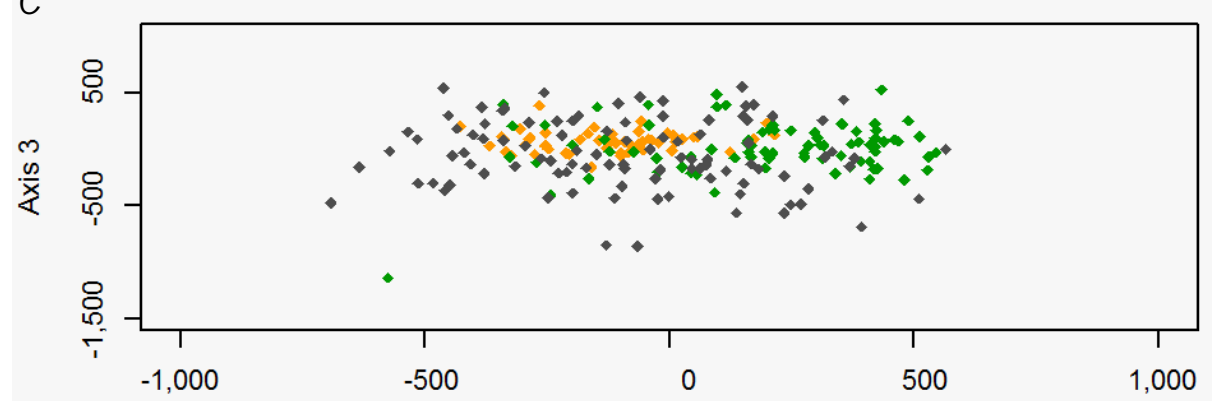

Axis 2

Figure 17. Factorial correspondence analysis results for black bears within the White River Basin (orange) in Arkansas and the Tensas River Basin north of Interstate 20 (green) and Tensas River Basin south of Interstate 20 (gray) in Louisiana: A, Axis 1 versus Axis 2; B, Axis 1 versus Axis $3 ; C$, Axis 2 versus Axis 3.

Similar to between-population genetic structure patterns, FCA revealed differing levels of within-population genetic structure among the four Louisiana populations. Within the TRB, a low level of structure was evident between bears sampled in the north and bears sampled in the south of the study area (fig. 18). Additionally, two bears did not cluster with the overall group (axis 2 versus axis 3 of the FCA, fig. 18) and were identified as outliers potentially having ancestry from another population. Bears in the TRC were strongly segregated into two genetic groups that did not correspond to any particular spatial pattern or landscape feature (fig. 19). Bears in the UARB did not show any evidence of genetic structure, except for one bear that was an extreme outlier (fig. 20). FCA revealed evidence of genetic structure in the LARB that corresponds to an eastern cluster and a western cluster with State Highway 317 and adjacent poor quality habitat operating as a potential movement barrier; this was also found by Troxler (2013; figs. 21-22). 


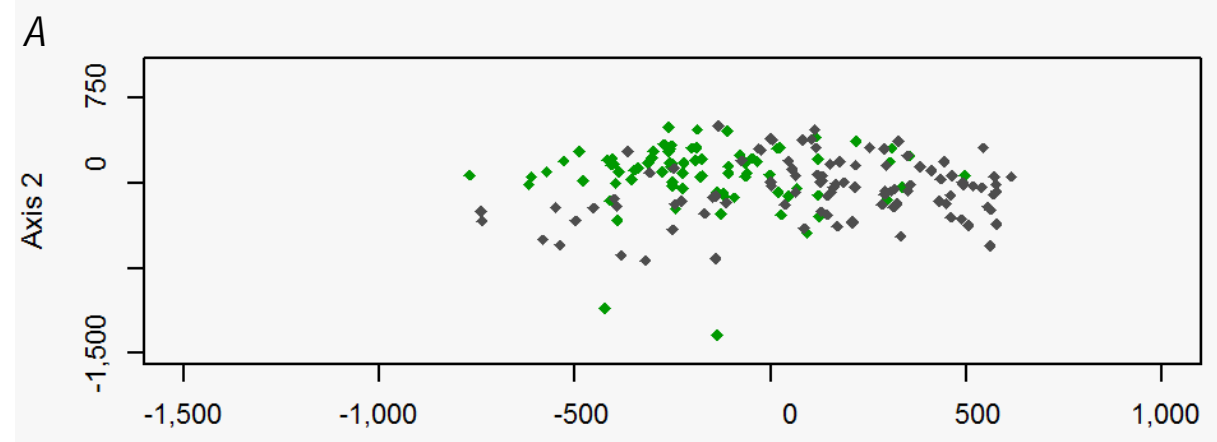

$B$

Axis 1

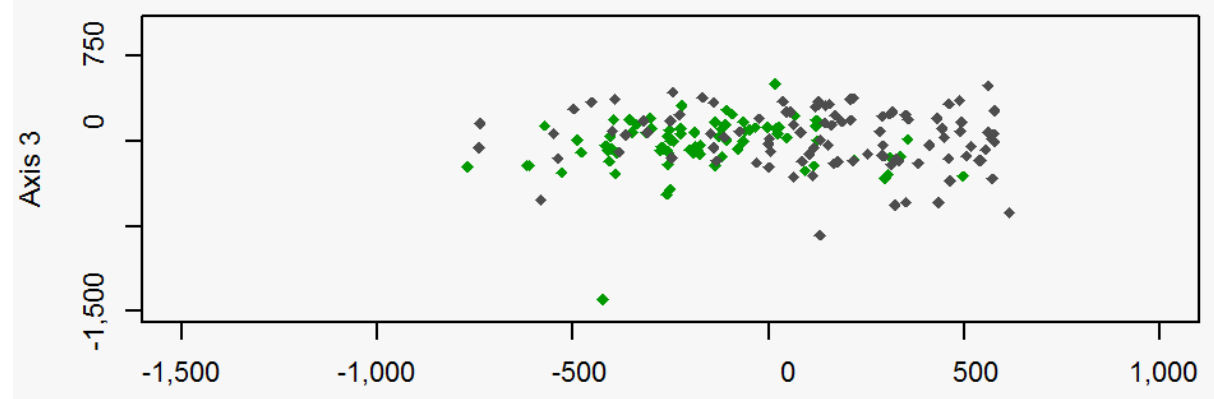

C

Axis 1

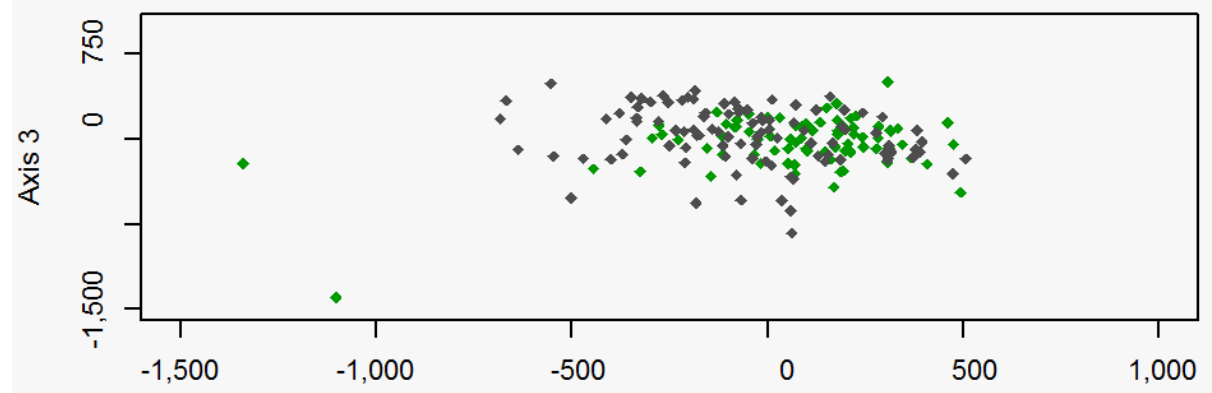

Axis 2

Figure 18. Factorial correspondence analysis results for black bears within the Tensas River Basin north of Interstate-20 (green) and south of Interstate-20 (gray) in Louisiana: A, Axis 1 versus Axis 2; B, Axis 1 versus Axis 3; C, Axis 2 versus Axis 3. 
A

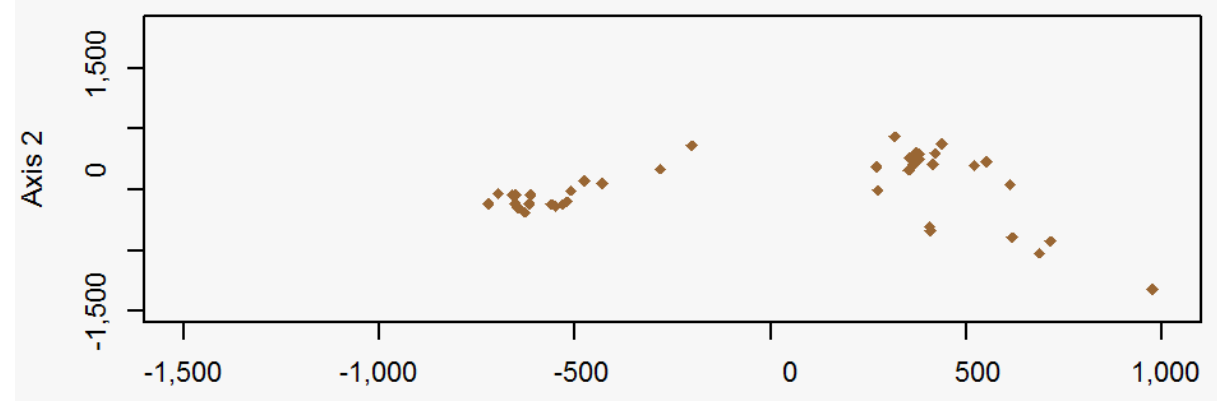

Axis 1

$B$

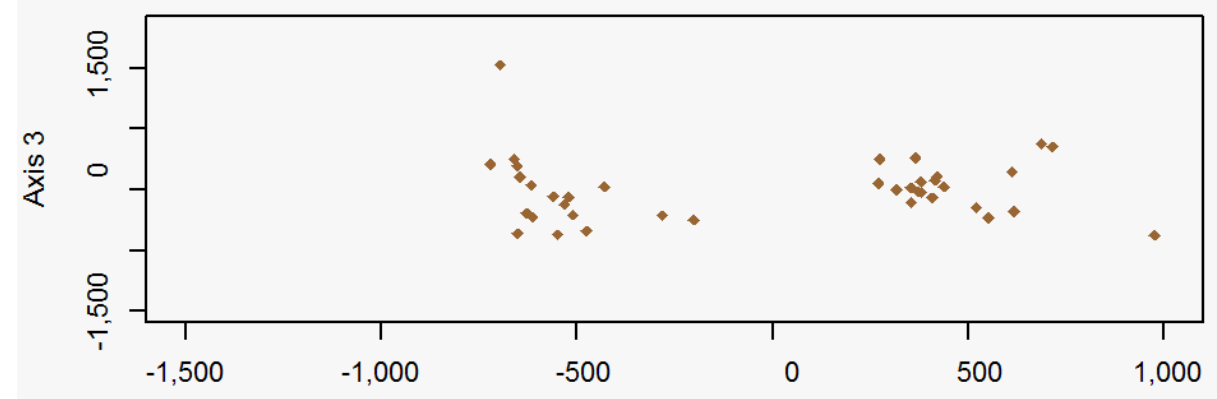

C

Axis 1

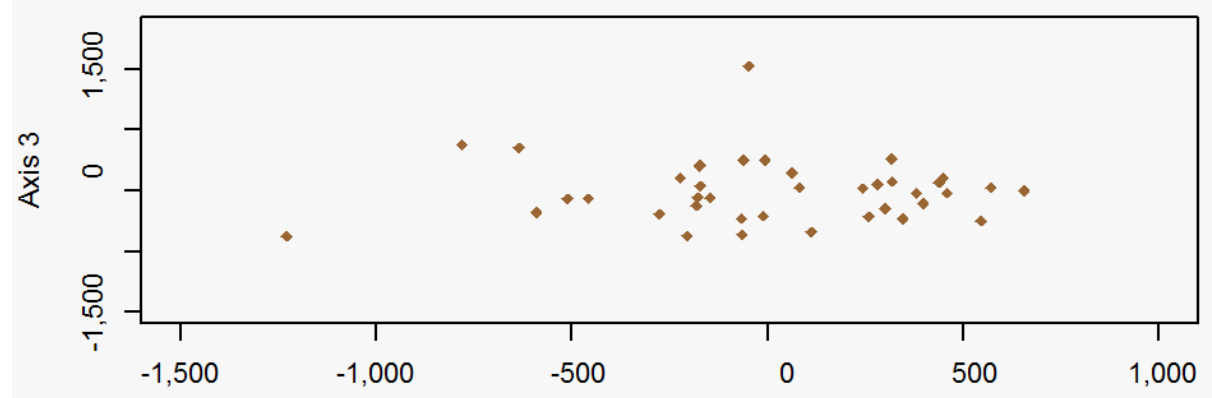

Axis 2

Figure 19. Factorial correspondence analysis results for black bear cubs born within the Three Rivers Complex in Louisiana: A, Axis 1 versus Axis 2; $B$, Axis 1 versus Axis 3; $C$, Axis 2 versus Axis 3. 


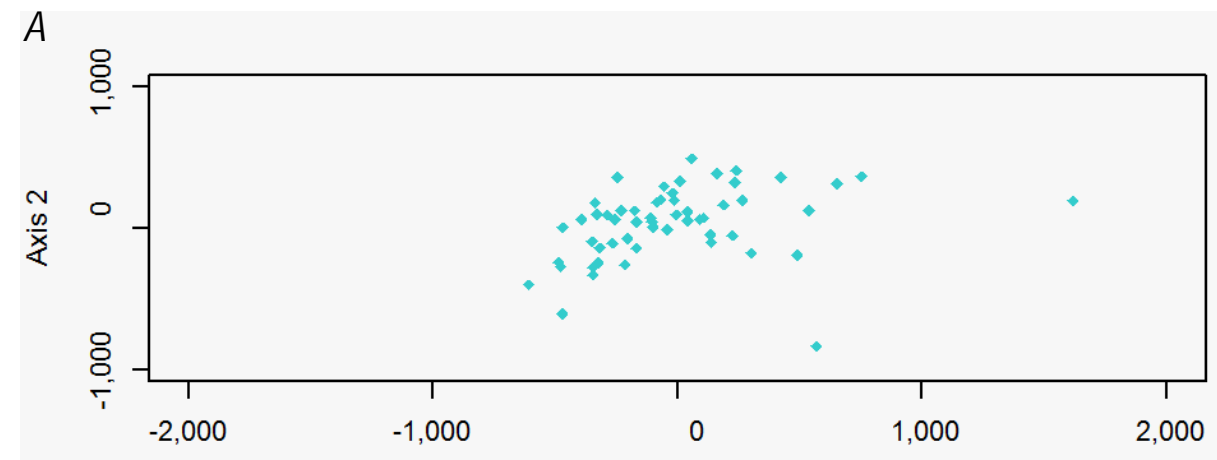

Axis 1
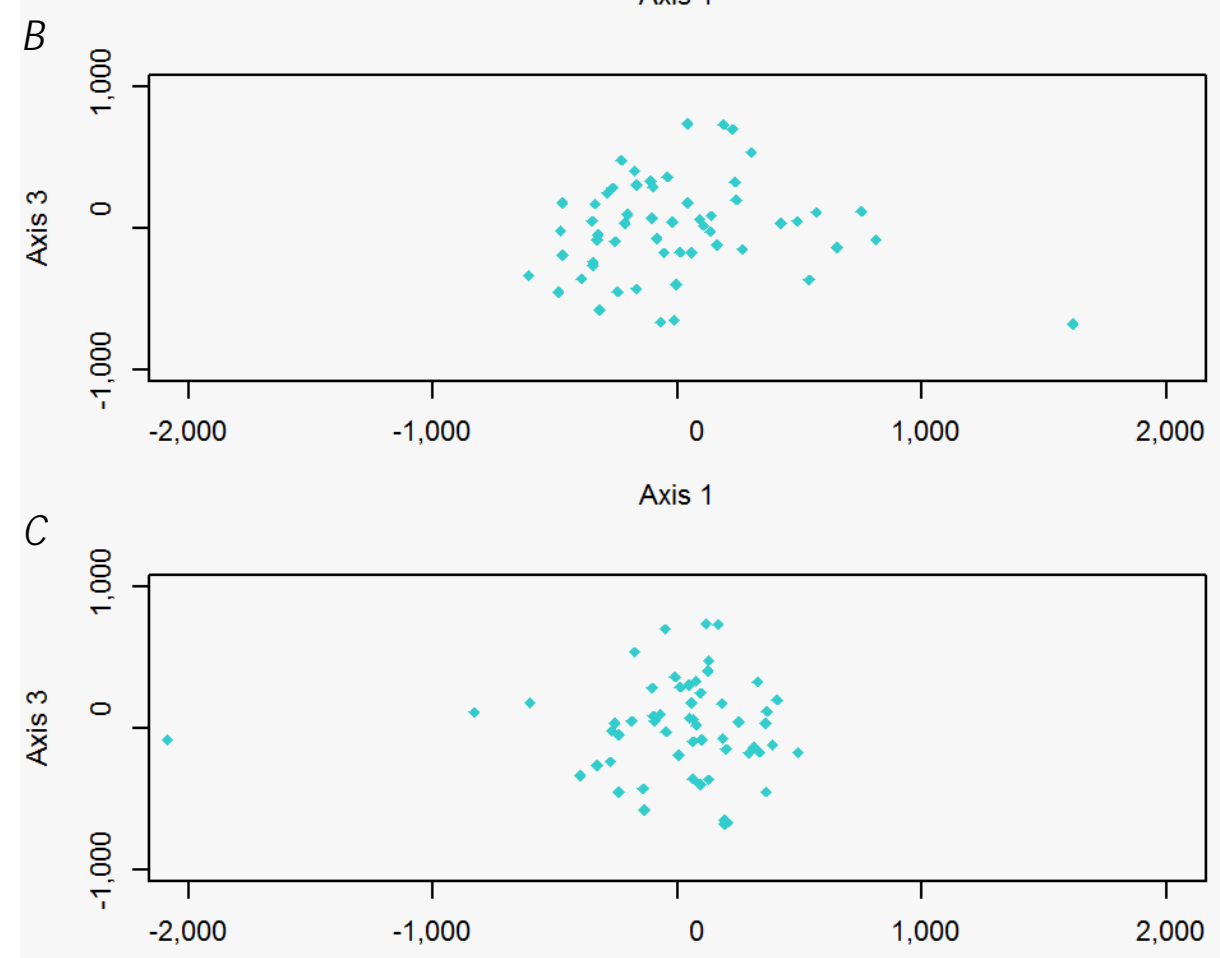

Axis 2

Figure 20. Factorial correspondence analysis results for black bears within the Upper Atchafalaya River Basin in Louisiana: A, Axis 1 versus Axis 2; $B$, Axis 1 versus Axis 3; $C$, Axis 2 versus Axis 3. 


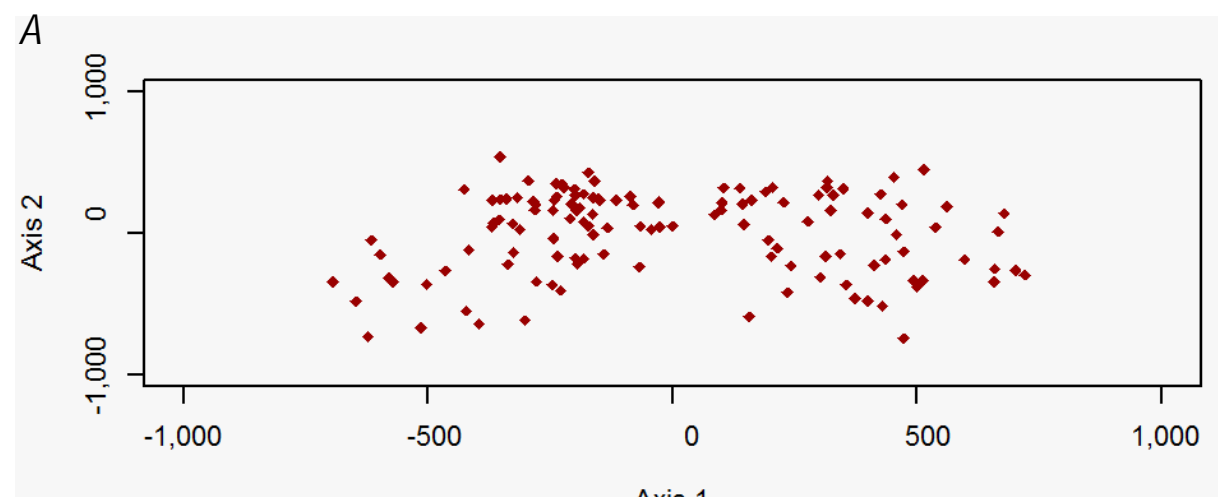

$B$

Axis 1

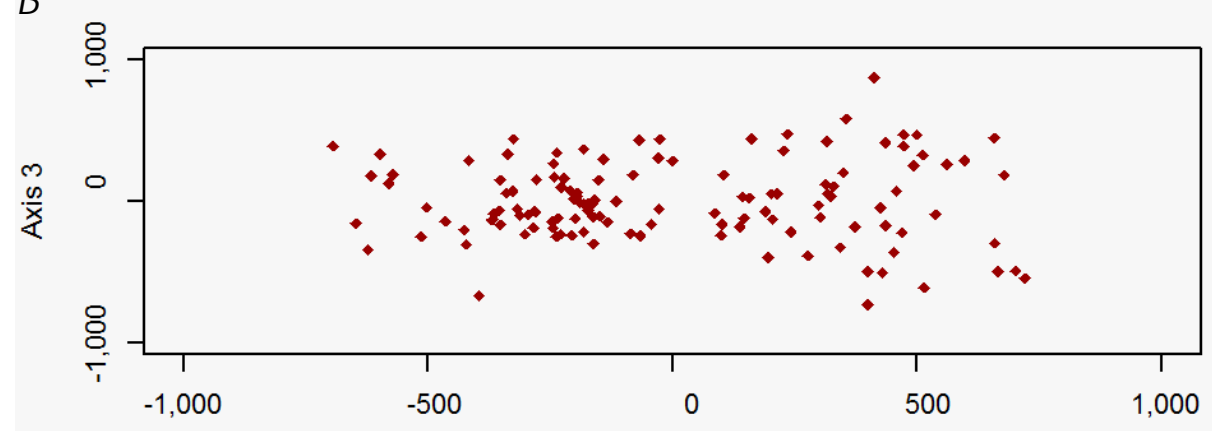

Axis 1

C

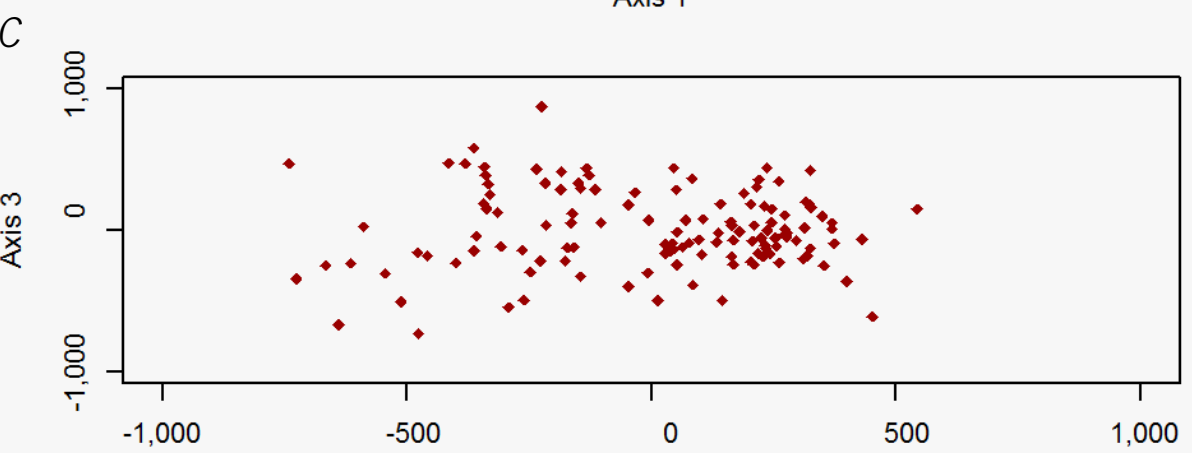

Axis 2

Figure 21. Factorial correspondence analysis results for bears within the Lower Atchafalaya River Basin in Louisiana: A, Axis 1 versus Axis 2; $B$, Axis 1 versus Axis 3; $C$, Axis 2 versus Axis 3. 


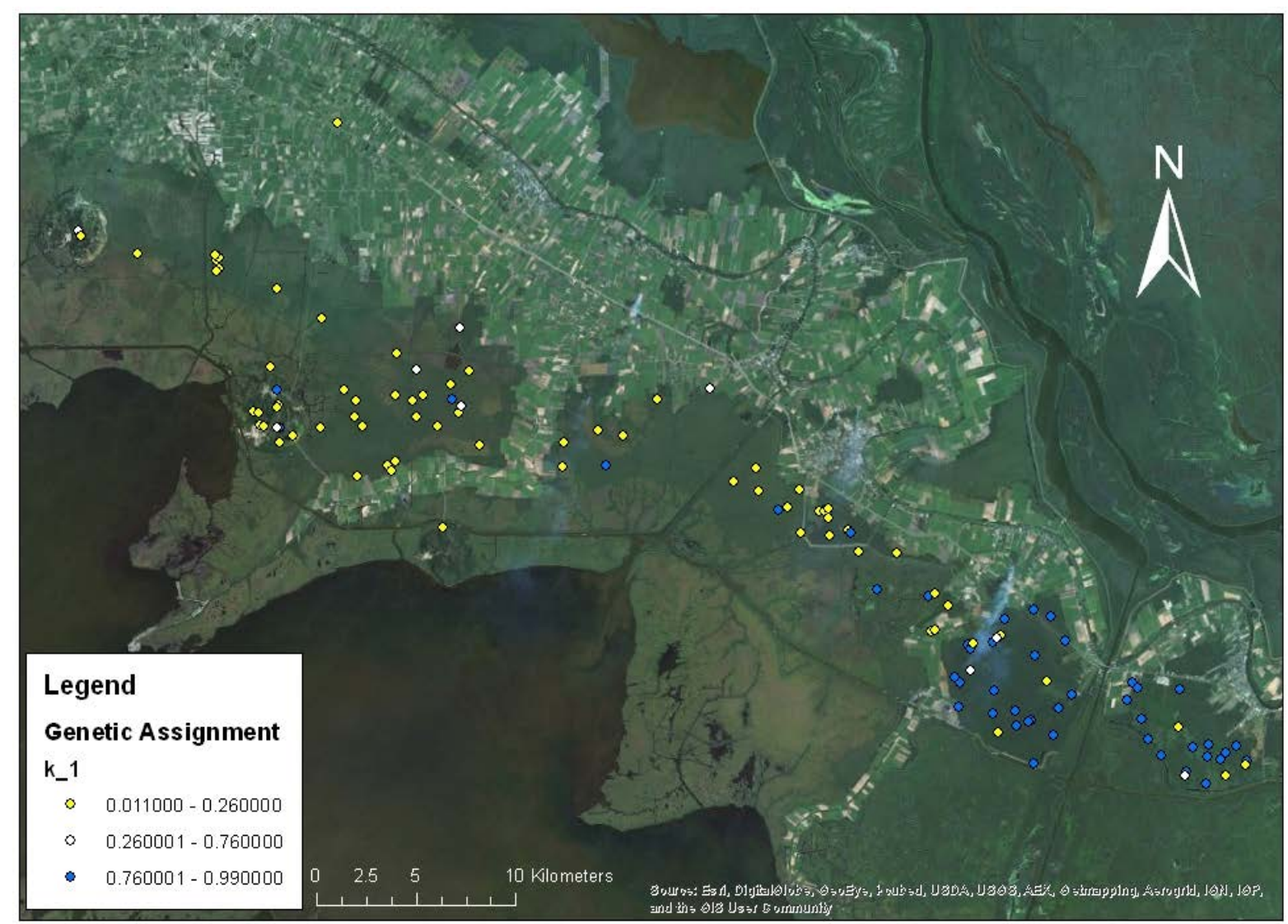

Figure 22. Mean capture locations from DNA-based captures for black bears in Lower Atchafalaya River Basin, Louisiana, 2010-12. From Troxler 2013. (Blue, greater than (>) 0.75 assignment to population 1; gold, $>0.75$ assignment to population 2; white, $0.25-0.75$ assignment to either population.

Clustering results from Program STRUCTURE consistently partitioned individuals into groups corresponding to known extant populations across independent MCMC chains as $K$ increased from 2 to 4 (fig. 23). At $K=2$, bears were partitioned into a group mostly consisting of individuals from WRB, TRB, and MISS and a group of individuals from MINN, UARB, and LARB. When $K$ was increased to 3 , bears in the LARB formed a single, separate cluster. When $K=4$, bears from WRB and a majority of MISS bears were clustered into a single group. At $K=5$, results across chains were less consistent. Six of 10 chains converged on population clusters corresponding to known populations in MINN, WRB, TRB, UARB, and LARB. Three chains pooled MINN and UARB together and split TRB into 2 groups, and 1 chain pooled MINN and UARB and split LARB into 2 groups. Results were inconclusive when $K$ $>5$ because of substantial variation of convergence among MCMC chains for each value of $K$. 


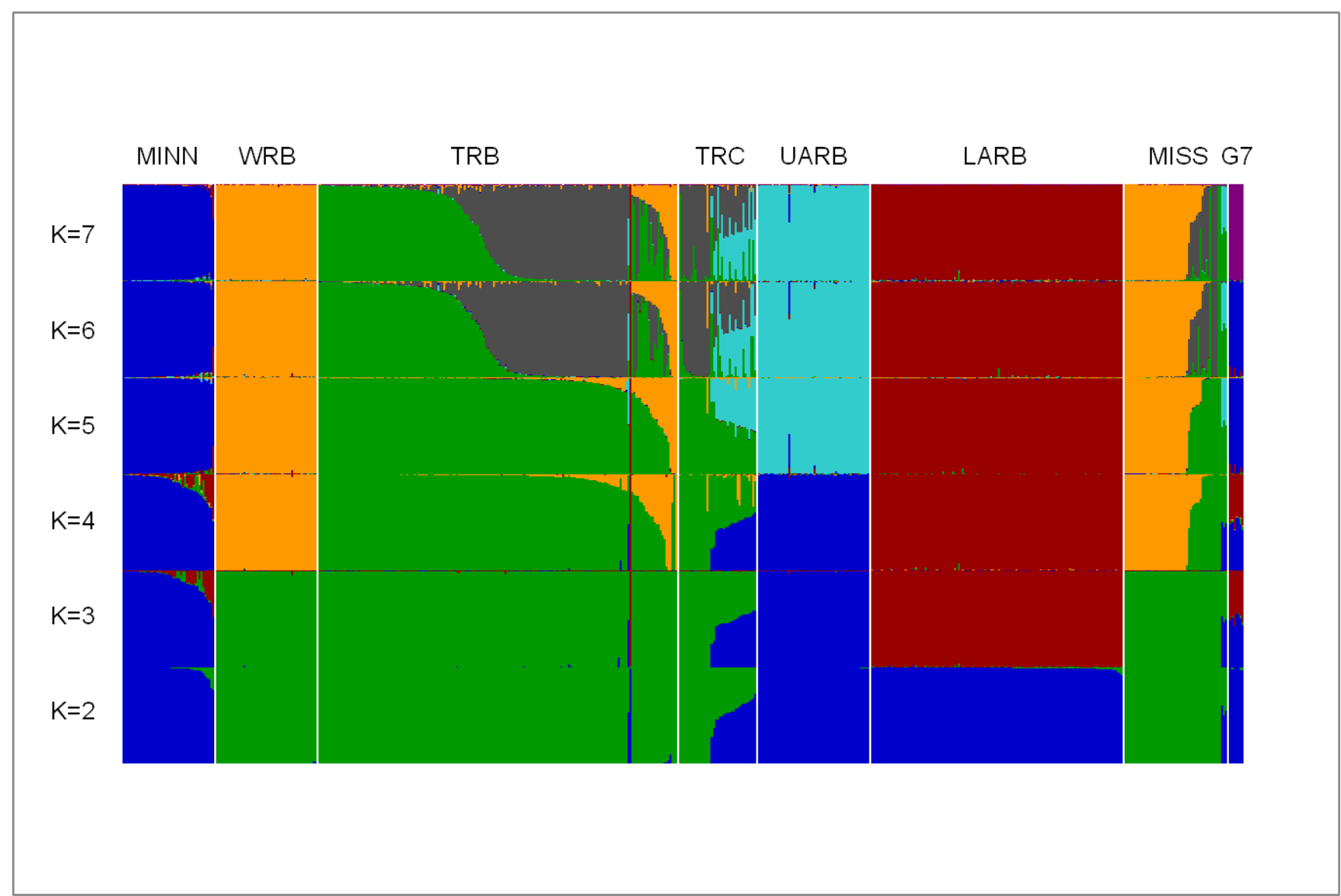

Figure 23. Proportional population ancestries for 556 black bears from Minnesota (MINN), western Mississippi (MISS), southeast Mississippi (G7), White River Basin in Arkansas (WRB), and Tensas River Basin (TRB), Three Rivers Complex (TRC), Upper Atchafalaya River Basin (UARB), and Lower Atchafalaya River Basin (LARB) in Louisiana, 2006-12. Ancestries were estimated using models in Program STRUCTURE based on assumed values of $\mathrm{K}$ (number of genetic clusters) that ranged from 2 to 11.

In general, $\log [K]$ values increased as $K$ was increased from 2 to 7 , after which values from different chains of $K$ greater than 7 began to overlap, indicating models with $K>7$ over fit our data (fig. 24). Based on $\Delta \log [K]$, the most likely number of populations present in the data was $K=4$ (fig. 25), which was also the value of $K$ that had the greatest and most stable values of $\log [K]$ across chains. However, most of the chains $(n=6)$ for $K=5$ converged on clusters corresponding to distinct populations known to be spatially segregated by large distances, indicating reasonable statistical support from the data. In total, measures of model fit and spatial distribution of fragmented populations indicate the most likely number of genetically distinct groups is five. 


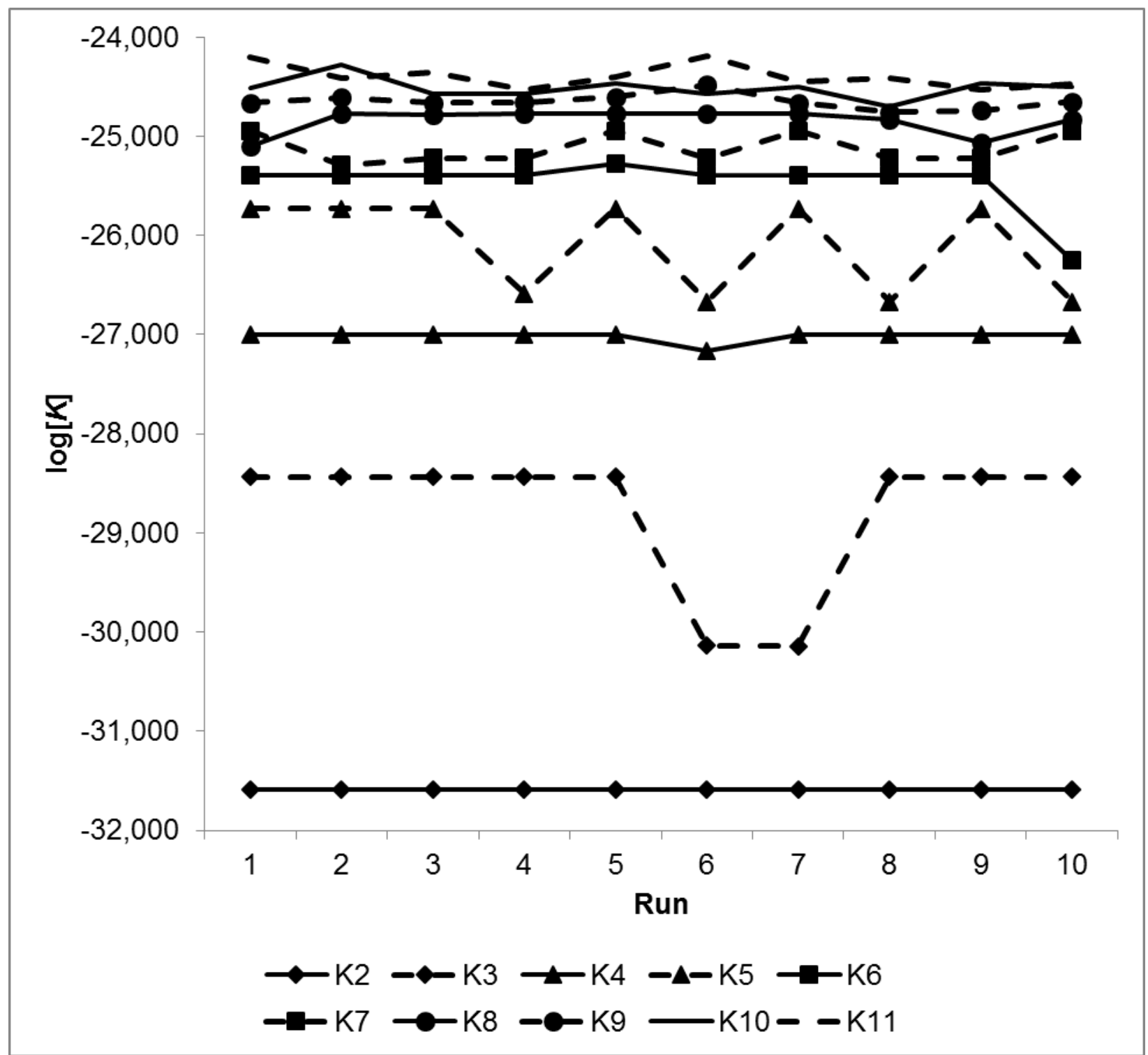

Figure 24. Program STRUCTURE $\log [K]$ values across 10 chains for each value of $K$ (number of genetic clusters) from 2 to 11, for Louisiana black bears in Louisiana, 2006-12. 


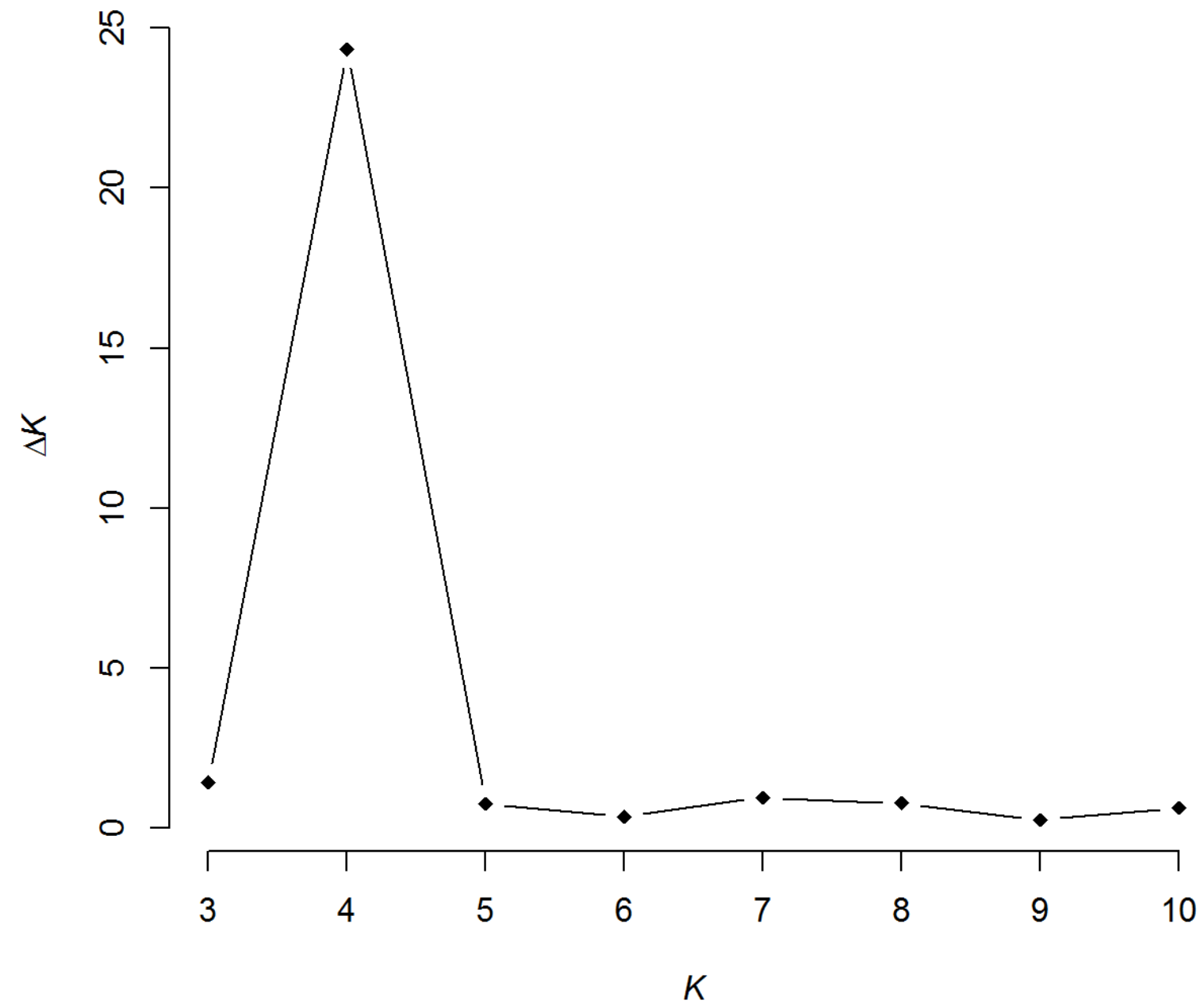

Figure 25. Estimated $\Delta \log [K]$ values from Program STRUCTURE population clustering analyses for values of $K$ (number of genetic clusters) from 3 to 10 for Louisiana black bears in Louisiana, 2006-12. 
On the basis of results from the FCA and preceding Program STRUCTURE analysis, an additional 10 chains were run in Program STRUCTURE for the $K=5$ model. For each bear, we then plotted the estimated probability that it had originated from each of the five putative clusters in search of evidence of genetic interchange between populations within the LMAV. In the TRB, nearly 30 bears had a $\geq 0.10$ probability of originating from WRB, 1 had a 0.99 probability of originating from the LARB, and 1 had a 0.48 probability of originating from the UARB (fig. 26). Thirty-two bears sampled in northwestern MISS had probabilities of WRB origin $\geq 0.90$, whereas only 10 bears from that area had $\mathrm{a} \geq 0.90$ probability of originating from the TRB (fig. 27). Six bears from northwestern MISS had mixed ancestry between WRB and TRB and were sampled east of the TRB and across the Mississippi River (fig. 27). Moreover, three cubs sampled in west-central MISS east of the TRC showed evidence of mixed ancestry between TRB and UARB. Of the sampled cubs born in the TRC, about one-half had mixed ancestry of TRB and UARB, and the other one-half had nearly complete TRB ancestries. Furthermore, an adult female reintroduced to the TRC that subsequently dispersed to the Boeuf Wildlife Management Area (northwest of the TRC and southwest of the TRB) and a cub of that female subsequently born at that location showed evidence of partial ancestry originating from WRB (upper portion of fig. 28). Thus, evidence shows that bears with WRB genes were present beyond TRB but not quite to TRC. 


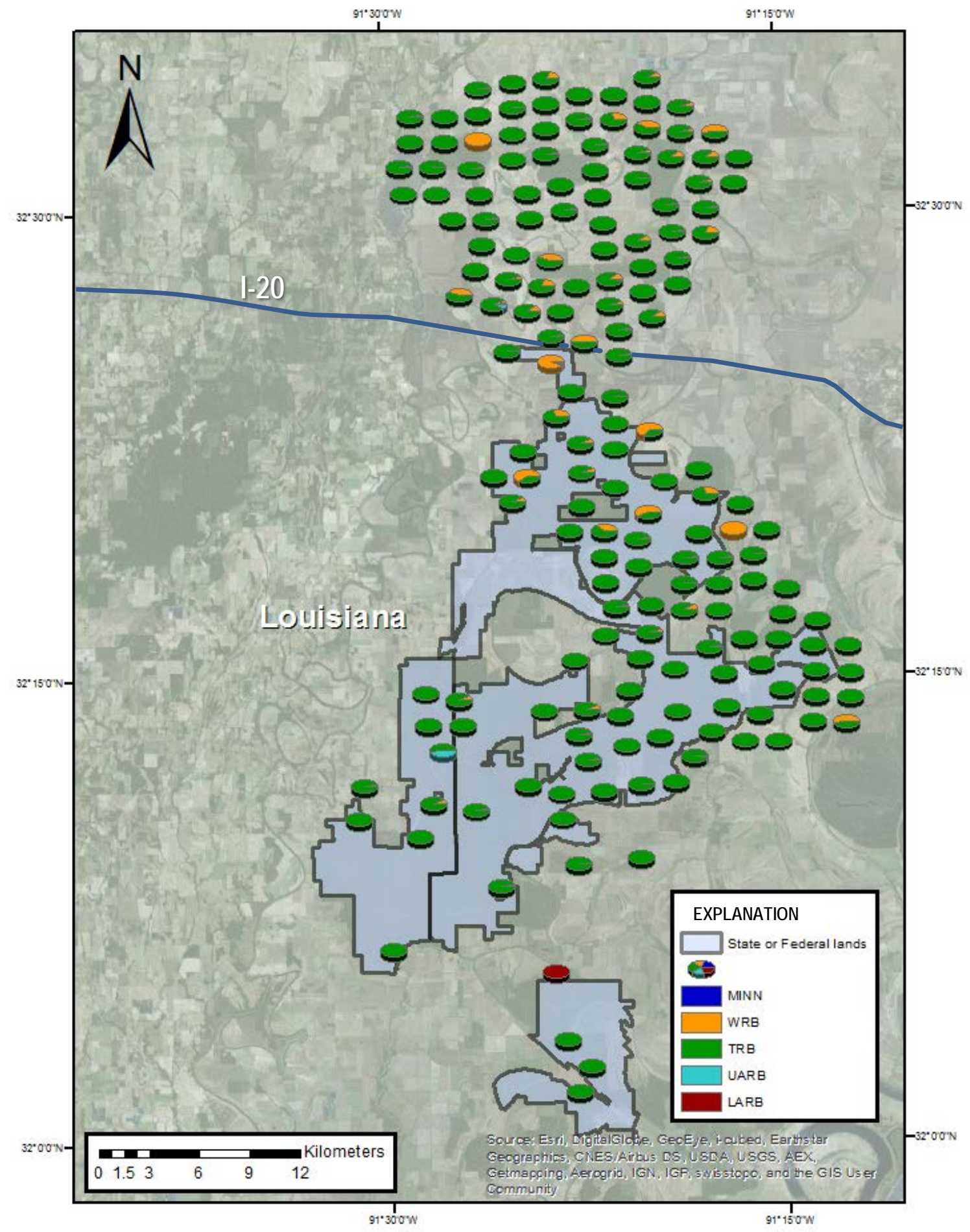

Figure 26. Proportional population ancestries for black bears within the Tensas River Basin in Louisiana, 200612, assigned to populations in Minnesota (MINN), White River Basin (WRB), Tensas River Basin (TRB), Upper Atchafalaya River Basin (UARB), or Lower Atchafalaya River Basin (LARB). Ancestries were estimated in Program STRUCTURE on the basis of an assumed value of $K=5$. ( $K$, number of genetic clusters; $I-20$, interstate 20.) 


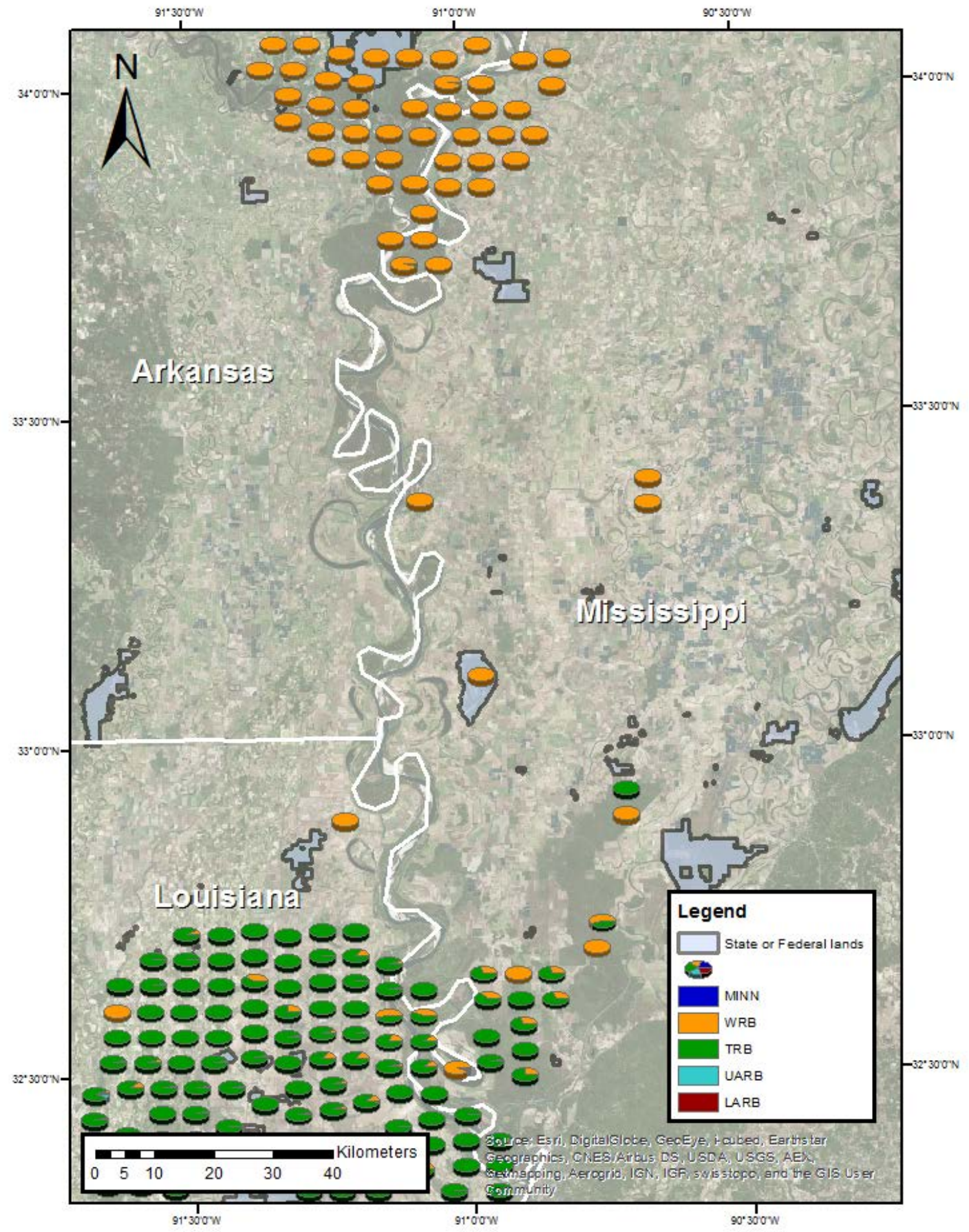

Figure 27. Proportional population ancestries for black bears within the southern portion of the White River Basin in Arkansas, the northern portion of the Tensas River Basin in Louisiana, and northwestern Mississippi, 2006-12, assigned to populations in Minnesota (MINN), White River Basin (WRB), Tensas River Basin (TRB), Upper Atchafalaya River Basin (UARB), or Lower Atchafalaya River Basin (LARB). Ancestries were estimated in Program STRUCTURE on the basis of an assumed value of $K=5$. ( $K$, number of genetic clusters.) 


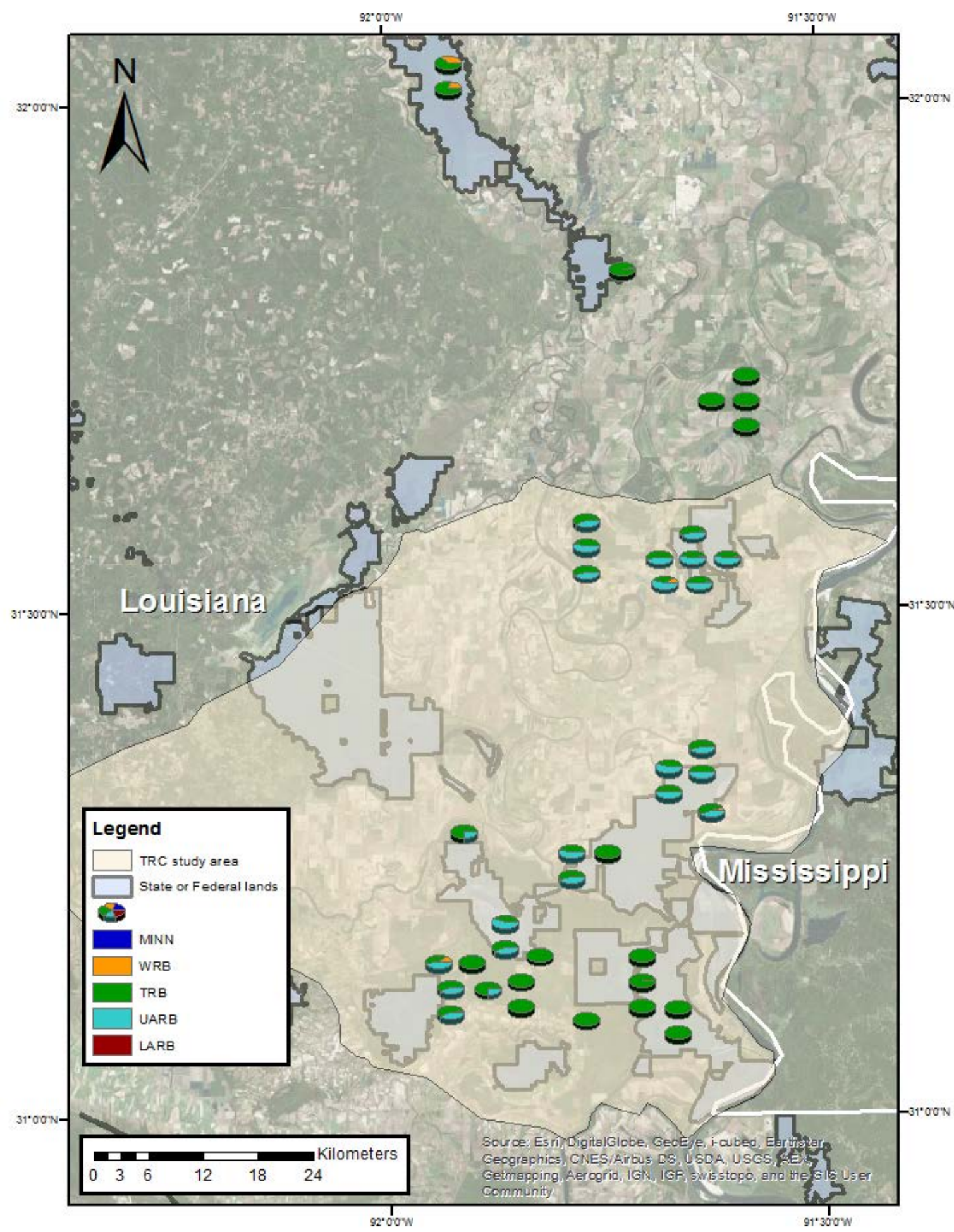

Figure 28. Proportional population ancestries for black bears within the Three Rivers Complex (TRC) in Louisiana, 2006-12, assigned to populations in Minnesota (MINN), White River Basin (WRB), Tensas River Basin (TRB), Upper Atchafalaya River Basin (UARB), or Lower Atchafalaya River Basin (LARB). Ancestries were estimated in Program STRUCTURE on the basis of an assumed value of $K=5$. (K, number of genetic clusters.) 


\section{Migrant Analysis Based on Genetic Data}

The search for migrants using Program GeneClass 2.0 identified 5 bears -4 males and 1 female - in the TRB as migrants from the WRB and 1 female bear in the TRB as a migrant from the LARB. No migrants were detected in the WRB, UARB, or LARB. The Program STRUCTURE-based migrant search using a model with $K=5$ identified the same female in the TRB as a migrant from LARB with a probability $>0.99$. Two males in the TRB were classified as migrants from the WRB with probabilities $>0.99$, and 1 male was classified as a true WRB migrant with a 0.60 probability. Taken together, results from both analyses identified 3 male migrants in TRB originating from WRB and 1 female migrant in TRB originating from the LARB. Twenty of 35 TRC cubs showed evidence of having been sired by immigrant males from UARB. Those cubs were distributed across 8 litters produced by 6 different females. Subsequently, a 2-year-old male PIT tagged as a cub at the UARB was captured in August 2014 at the TRC, providing evidence of successful male dispersal (Maria Davidson, LDWF, written commun., 2014). Searches of DNA-based CMR histories and live-capture records identified three males in the TRB that dispersed from the TRC. One male was a cub born to and moved with a female in 2006 that was translocated from the TRB to the TRC as part of the reintroduction. That male was subsequently live captured in the TRB in 2010. The other two males were born in the TRC to translocated females, handled as cubs in their natal dens, and subsequently detected at hair-collection sites in the TRB by age 2. A fourth male was detected at hair-collection sites in the TRB and was classified as a second generation migrant from the UARB (that is, 1 parent from the UARB). Three females were detected at hair-collection sites in the WRB from 2004 to 2006 and were subsequently live captured 80-150 km south of the WRB in Mississippi between 2008 and 2010. In July 2012, a GPScollared male originally captured in the UARB in 2011 traveled from the UARB to the southern part of the TRC and back to the UARB. In 2014, a 2.5-year-old male born in the UARB was captured approximately $90 \mathrm{~km}$ north in the TRC. Finally, 1 male was detected at hair-collection sites in the TRB in 2007 and was later live captured approximately $14 \mathrm{~km}$ directly east of the TRB in Mississippi, and 1 female was translocated to the TRC in 2005 as part of the reintroduction effort and subsequently dispersed to Mississippi, directly east of the TRC.

\section{GPS Telemetry Data to Estimate Successful Interchange Rates and Paths}

From April 2010 to April 2012, 8 female and 23 male bears ranging from 1 to 11 years of age were radio collared (table 7). Most of the bears were young; 4 of the females and 18 of the males were $\leq 3$ years of age. From April 2010 to October 2012, 5,400 GPS fixes were obtained for females and 30,832 for males. After generating steps from sequential parings and eliminating those that did not meet our inclusion criteria, 2,104 and 15,233 steps with median length $391.6 \mathrm{~m}$ and $649.4 \mathrm{~m}$ for females and males, respectively, were retained. The mean interval between GPS fixes was 2.71 hours for females and 2.78 hours for males. Step lengths for both sexes were skewed toward shorter distances, but extreme step lengths were more common for males (fig. 29). Turning angles were most frequent around 0 degrees, meaning that bears of both sexes exhibited directional persistence and a secondary tendency for steps to return in the direction from which they came (fig. 30). Turning angles and step lengths were independent for both sexes (correlation coefficient $\left[R^{2}\right]<0.001$ ). Path lengths for females during the period of monitoring (that is, 25.2 to 804.8 days, mean $[\bar{x}]=241.4)$ ranged from 32.9 to $419.1 \mathrm{~km}(\bar{x}=$ $185.5)$ and for males from 73.5 to $2,670.2 \mathrm{~km}(\bar{x}=623.7)$. The Euclidean distances moved from the beginning to end point of a path (displacement) ranged from 0.6 to $6.5 \mathrm{~km}(\bar{x}=3.6)$ for females and from 0.5 to $88.5 \mathrm{~km}(\bar{x}=11.2)$ for males. The maximum distance from the point of origin ranged from 3.1 to $10.8 \mathrm{~km}(\bar{x}=7.3)$ for females and 4.0 to $88.5 \mathrm{~km}(\bar{x}=18.2)$ for males. 
Table 7. Global Positioning System (GPS) collars deployed on bears in Louisiana, 2010-12.

[M, male; F, female]

\begin{tabular}{|c|c|c|c|c|c|}
\hline Bear identity & $\begin{array}{c}\text { Collar retrieval or Satellite } \\
\text { data }\end{array}$ & Sex & Age & Deployment date & Last movement date \\
\hline Nstar1 & Satellite & M & 3 & $5 / 12 / 2010$ & $10 / 15 / 2010$ \\
\hline Nstar3 & Satellite & M & 4 & $5 / 16 / 2010$ & 8/24/2011 \\
\hline Nstar4 & Satellite & M & 3 & $4 / 24 / 2010$ & 8/9/2011 \\
\hline Nstar5 & Collar/Satellite & M & 2 & $4 / 18 / 2010$ & 8/6/2011 \\
\hline Nstar6 & Satellite & M & 2 & $7 / 3 / 2010$ & $5 / 21 / 2011$ \\
\hline Nstar7 & Collar & $\mathrm{F}$ & 3 & $6 / 15 / 2010$ & $10 / 13 / 2010$ \\
\hline Nstar8 & Collar & M & 1 & 6/9/2010 & 7/31/2010 \\
\hline Nstar9 & Collar & M & 2 & $10 / 6 / 2010$ & $12 / 14 / 2010$ \\
\hline Nstar10 & Collar & M & 2 & $6 / 16 / 2010$ & 8/30/2012 \\
\hline Nstar12 & Collar & M & $1-2$ & $6 / 5 / 2011$ & 4/27/2012 \\
\hline Nstar13 & Satellite & M & 2 & 6/16/2010 & $10 / 8 / 2010$ \\
\hline Nstar14 & World Wide Web & M & 3 & $6 / 10 / 2010$ & 7/19/2011 \\
\hline Nstar16 & Collar & M & 3 & 5/31/2010 & $12 / 1 / 2010$ \\
\hline Nstar17 & Collar & M & 1 & $6 / 13 / 2010$ & $10 / 15 / 2010$ \\
\hline Nstar18 & Collar & $\mathrm{F}$ & 3 & $6 / 26 / 2010$ & 7/22/2010 \\
\hline Nstar19 & Collar & M & 4 & $6 / 14 / 2010$ & $12 / 14 / 2010$ \\
\hline Nstar20 & Collar & $\mathrm{F}$ & 5 & 5/14/2010 & $11 / 14 / 2010$ \\
\hline Nstar21 & Satellite & M & 3 & 6/7/2011 & 8/18/2011 \\
\hline Telonics2 & Collar & M & 9 & 4/4/2012 & $7 / 24 / 2012$ \\
\hline Telonics69 & Collar & M & 3 & 7/2/2011 & 8/30/2012 \\
\hline Telonics71 & Collar & $\mathrm{F}$ & 2 & 10/16/2011 & 6/20/2012 \\
\hline Telonics79 & Collar & M & 5 & 6/21/2011 & 7/26/2012 \\
\hline Telonics80 & Collar & M & 3 & 6/30/2011 & 10/3/2012 \\
\hline Telonics84 & Collar & M & 2 & 7/15/2011 & 8/1/2012 \\
\hline Telonics375 & Collar & $\mathrm{F}$ & 11 & 4/16/2012 & 8/30/2012 \\
\hline Telonics711 & Collar & M & 2 & 9/27/2011 & 10/24/2011 \\
\hline Telonics713 & Collar & $\mathrm{F}$ & $8-10$ & 10/9/2011 & 4/19/2012 \\
\hline Telonics714 & Collar & $\mathrm{F}$ & 5 & $10 / 12 / 2011$ & 11/14/2011 \\
\hline Telonics790 & Collar & M & 5 & 4/17/2012 & 8/29/2012 \\
\hline Telonics831 & Collar & M & 3 & 4/2/2012 & 8/30/2012 \\
\hline
\end{tabular}


Step lengths for females

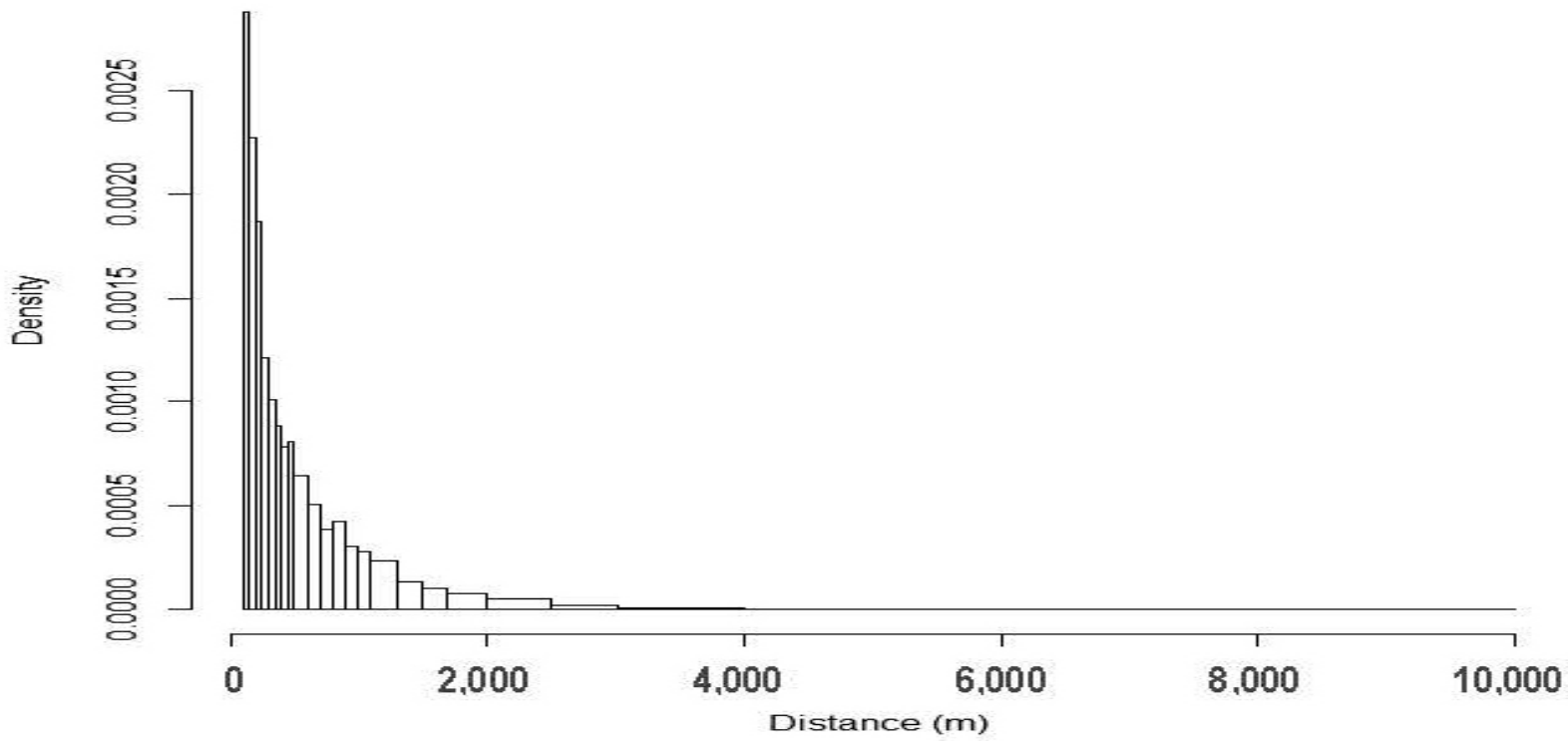

Step lengths for males

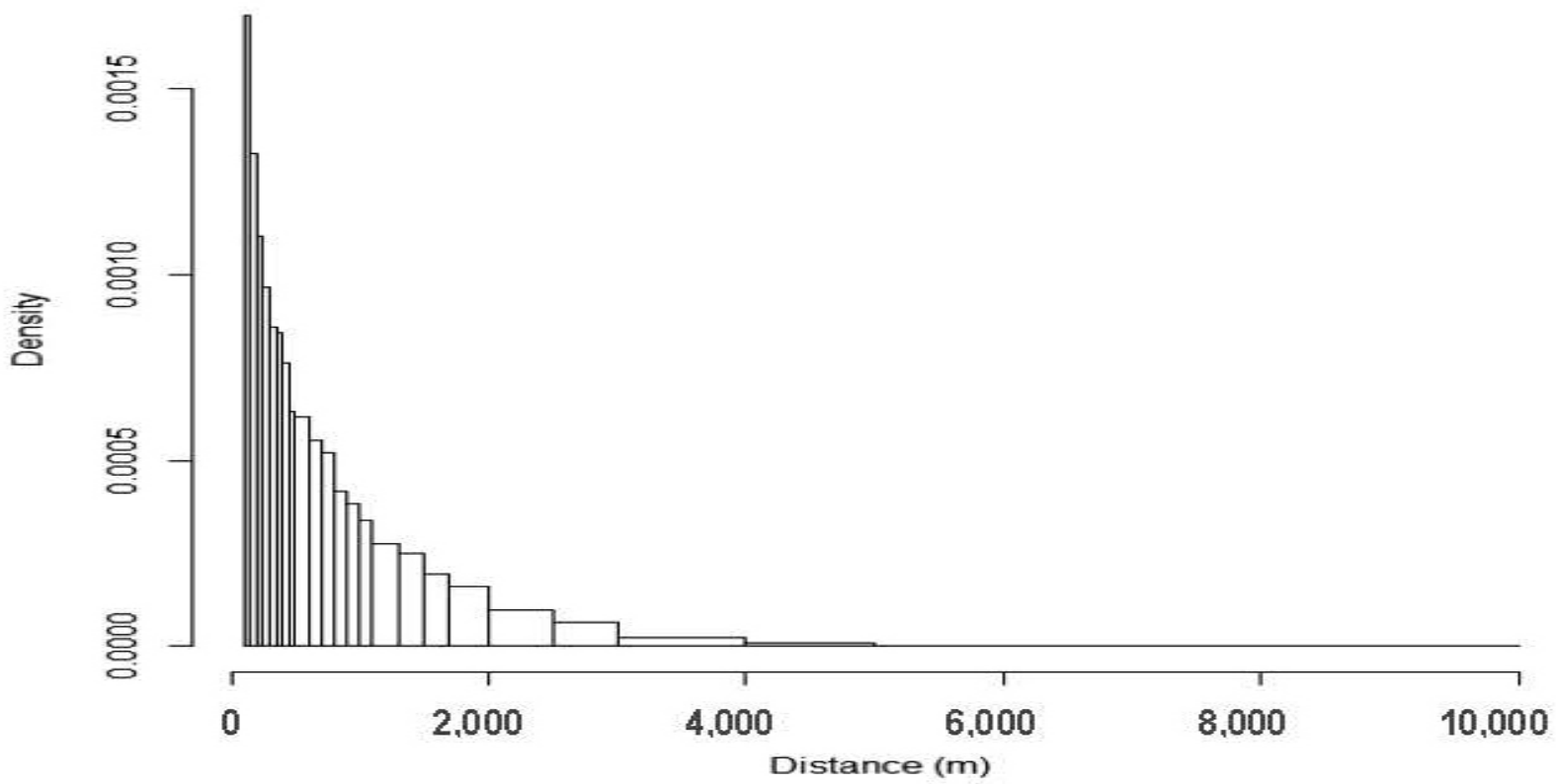

Figure 29. Step lengths for female and male Louisiana black bears based on GPS radio collar data collected in Louisiana, during 2010-12. (m, meters) 

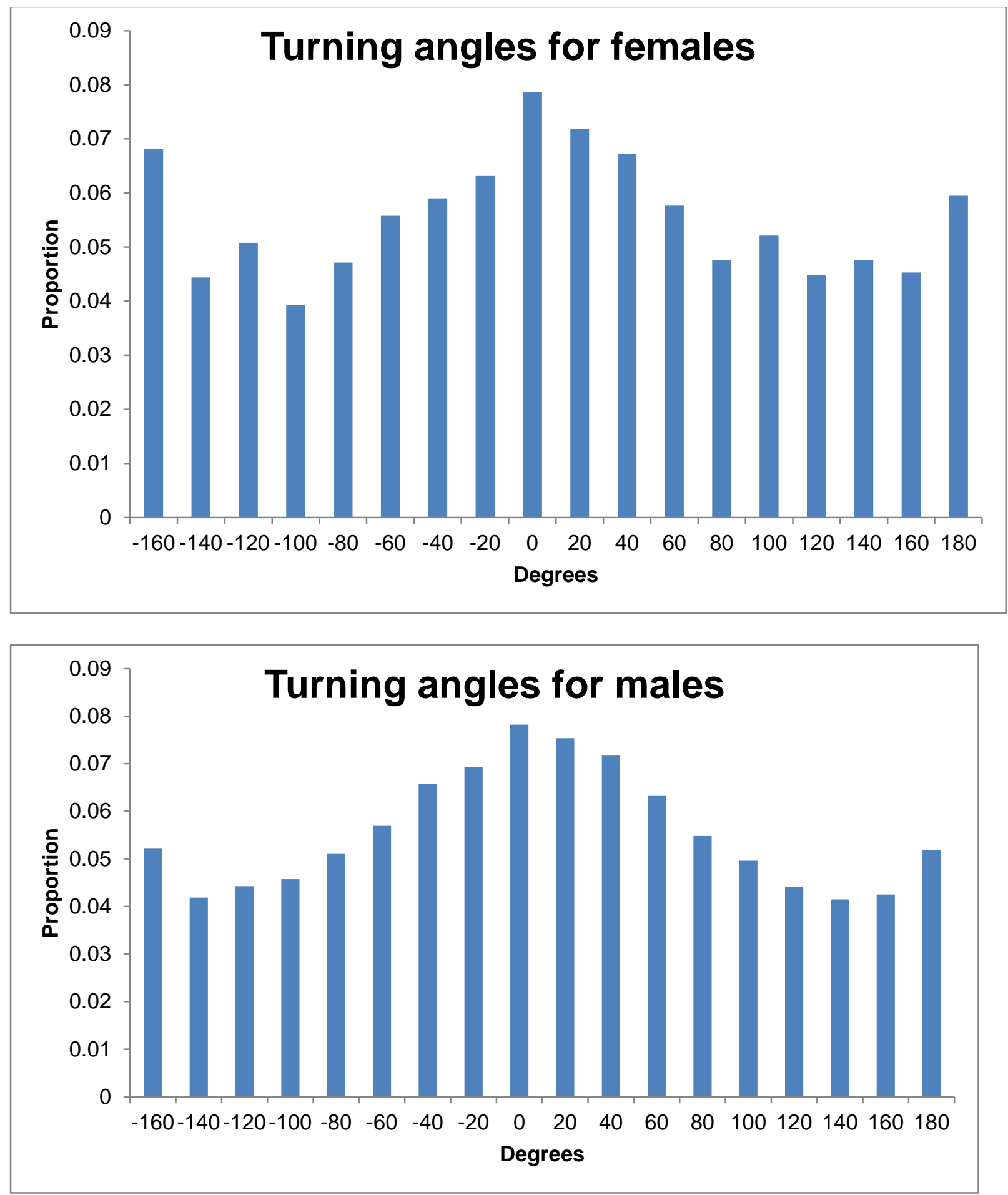

Figure 30. Turning angles for female and male Louisiana black bears based on GPS radio collar data collected in Louisiana, during 2010-12.

For females, the probability of a step being selected increased as the distance to natural land cover and distance to agriculture at the end of the step decreased and as the distance at the end of a step to roads increased. The remaining land cover variables (that is, percent forest, forest density, percent natural, natural density, percent agriculture, distance to water, road density, contagion, splitting index, 
and landscape shape index) were not retained. The probability of a step was also influenced by an interaction between distance to natural land cover and distance to roads, and an interaction between distance to natural land cover and distance to agriculture (table 8). If a bear was near natural land cover, the selection coefficient slightly increased as the distance to roads increased but decreased if far from natural land cover (fig. 31A). Selection decreased as the distance to agriculture increased but only if near natural land cover; otherwise, selection increased (fig 31B). The deviance reduction associated with the removal of each variable from the model indicates that distance to natural cover (-626.7) had the greatest effect on step selection followed by distance to roads (-71.5) and distance to agriculture (-13.4). A cutoff of 10 autocorrelated steps was identified, so the robust SE estimates were based on that cluster size. The pseudo $R^{2}(0.027$, maximum $=0.353)$ and the standardized $\beta$ values indicate that selection strength was generally low for all models (table 8). Although no attempt was made to incorporate temporal covariates into the path projections, an interaction was observed between distance to agriculture and season for females $\left(\beta=-3.15 \mathrm{E}-04, \mathrm{SE}_{\text {robust }}=2.83 \mathrm{E}-05\right)$, with selection declining during the growing season as distance to agriculture became greater but increasing during the non-growing season. On the basis of the mixed effects models, the relation between the probability of a selected step and the distance to the nearest natural land-cover type varied by individual $\left(\sigma^{2}=3.70 \mathrm{E}-05, P<0.001\right)$, with 93.1 percent expected to have a negative relation.

Table 8. Cox proportional hazards models used to create step-selection functions, Louisiana, 2010-12.

[ $\beta$, slope; SE, standard error; CI, confidence interval; --, not applicable; ID, bear identity; SD, standard deviation; P, probability; *, interaction effect; **, quadratic effect]

\begin{tabular}{|c|c|c|c|c|}
\hline Variable & $\beta$ & SE & $95 \% \mathrm{Cl}$ & Standardized $\beta$ \\
\hline \multicolumn{5}{|l|}{ Female model (fixed effects) } \\
\hline Distance to natural land cover (end) & $-4.74 \mathrm{E}-03$ & $1.26 \mathrm{E}-03^{\mathrm{a}}$ & $-7.21 \mathrm{E}-03--2.36 \mathrm{E}-03$ & -0.719 \\
\hline Distance to agriculture (end) & $-2.19 \mathrm{E}-04$ & $3.45 \mathrm{E}-05^{\mathrm{a}}$ & $-2.86 \mathrm{E}-04--1.51 \mathrm{E}-04$ & -0.194 \\
\hline Distance to roads (end) & $1.97 \mathrm{E}-05$ & $1.97 \mathrm{E}-05^{\mathrm{a}}$ & $-1.89 \mathrm{E}-05-5.82 \mathrm{E}-05$ & 0.038 \\
\hline Distance to natural*distance to roads & $-1.87 \mathrm{E}-06$ & $1.55 \mathrm{E}-07^{\mathrm{a}}$ & $-2.17 \mathrm{E}-06--1.56 \mathrm{E}-06$ & -1.325 \\
\hline Distance to natural*distance to agriculture & $1.95 \mathrm{E}-06$ & $4.62 \mathrm{E}-07^{\mathrm{a}}$ & $1.04 \mathrm{E}-06-2.85 \mathrm{E}-06$ & 0.605 \\
\hline \multicolumn{5}{|l|}{ Female model (mixed effects) } \\
\hline \multicolumn{5}{|l|}{ Fixed } \\
\hline Distance to agriculture (end) & $-2.06 \mathrm{E}-04$ & 8.62E-05 & $-3.75 \mathrm{E}-04--3.72 \mathrm{E}-05$ & -- \\
\hline Distance to roads (end) & 2.33E-06 & 5.64E-05 & $-1.08 \mathrm{E}-04-1.13 \mathrm{E}-04$ & -- \\
\hline Distance to natural*distance to roads & $-1.07 \mathrm{E}-06$ & $5.18 \mathrm{E}-07$ & $-2.09 \mathrm{E}-06--5.91 \mathrm{E}-08$ & -- \\
\hline Distance to natural*distance to agriculture & $1.75 \mathrm{E}-06$ & $8.72 \mathrm{E}-07$ & $3.84 \mathrm{E}-08-3.46 \mathrm{E}-06$ & -- \\
\hline \multicolumn{5}{|l|}{ Random } \\
\hline Distance to natural | bear ID & $-9.01 E-03$ & 2.71E-03 & $-1.43 \mathrm{E}-02--3.70 \mathrm{E}-03$ & -- \\
\hline SD of coefficient & 6.09E-03 & -- & -- & -- \\
\hline Maximum log likelihood & -4649.8 & -- & -- & -- \\
\hline Likelihood ratio test & $P<0.001$ & -- & -- & -- \\
\hline \multicolumn{5}{|l|}{ Male model (fixed effects) } \\
\hline Distance to natural land cover (end) & $-6.61 E-03$ & $3.18 \mathrm{E}-04^{\mathrm{a}}$ & $-7.23-03--5.99 E-03$ & -1.083 \\
\hline Distance to agriculture (end) & $-1.67 \mathrm{E}-04$ & $2.49 \mathrm{E}-05^{\mathrm{a}}$ & $-2.16 \mathrm{E}-04--1.18 \mathrm{E}-04$ & -0.123 \\
\hline Distance to roads (end) & $2.50 \mathrm{E}-04$ & $5.77 \mathrm{E}-06^{\mathrm{a}}$ & $2.38 \mathrm{E}-04--2.61 \mathrm{E}-04$ & 0.400 \\
\hline Distance to natural $* * 2$ & 3.05E-06 & $1.34 \mathrm{E}-06^{\mathrm{a}}$ & $4.26 \mathrm{E}-07-5.68 \mathrm{E}-06$ & 0.601 \\
\hline Distance to roads $* * 2$ & $-3.58 E-08$ & $4.97 \mathrm{E}-09^{\mathrm{a}}$ & $-4.55 \mathrm{E}-08--2.60 \mathrm{E}-08$ & -0.359 \\
\hline Distance to natural*distance to agriculture & $-1.09 \mathrm{E}-06$ & $7.26 \mathrm{E}-07^{\mathrm{a}}$ & $-2.51 \mathrm{E}-06-3.36 \mathrm{E}-07$ & -0.425 \\
\hline
\end{tabular}


Table 8. Cox proportional hazards models used to create step-selection functions, Louisiana, 2010-12.Continued

[ $\beta$, slope; SE, standard error; CI, confidence interval; --, not applicable; ID, bear identity; SD, standard deviation; P, probability; *, interaction effect; **, quadratic effect]

\begin{tabular}{|c|c|c|c|c|}
\hline $\begin{array}{r}\text { Variable } \\
\end{array}$ & $\beta$ & SE & $95 \% \mathrm{Cl}$ & Standardized $\beta$ \\
\hline \multicolumn{5}{|l|}{ Male model (mixed effects) } \\
\hline \multicolumn{5}{|l|}{ Fixed } \\
\hline Distance to agriculture (end) & $-1.75 \mathrm{E}-04$ & 2.75E-05 & $-2.29 \mathrm{E}-04--1.21 \mathrm{E}-04$ & -- \\
\hline Distance to roads (end) & $2.48 \mathrm{E}-04$ & 2.91E-05 & $1.91 \mathrm{E}-04-3.05 \mathrm{E}-04$ & -- \\
\hline Distance to natural $* * 2$ & 2.89E-06 & 3.28E-07 & 2.25E-06 - 3.54E-06 & -- \\
\hline Distance to roads $* * 2$ & $-3.72 \mathrm{E}-08$ & 4.87E-09 & $-4.68 \mathrm{E}-08--2.77 \mathrm{E}-08$ & -- \\
\hline Distance to natural*distance to agriculture & $-1.16 \mathrm{E}-06$ & 1.99E-07 & $-1.56 \mathrm{E}-06--7.73 \mathrm{E}-07$ & -- \\
\hline \multicolumn{5}{|l|}{ Random } \\
\hline Distance to natural | bear ID & $-6.63 E-03$ & 5.52E-04 & $-7.71 \mathrm{E}-03--5.54 \mathrm{E}-03$ & -- \\
\hline SD of coefficient & 2.36E-03 & -- & -- & -- \\
\hline Maximum log likelihood & -34797.0 & -- & -- & -- \\
\hline Likelihood ratio test & $P<0.001$ & -- & -- & -- \\
\hline
\end{tabular}

${ }^{\mathrm{a}}$ Robust estimates accounting for autocorrelation. 

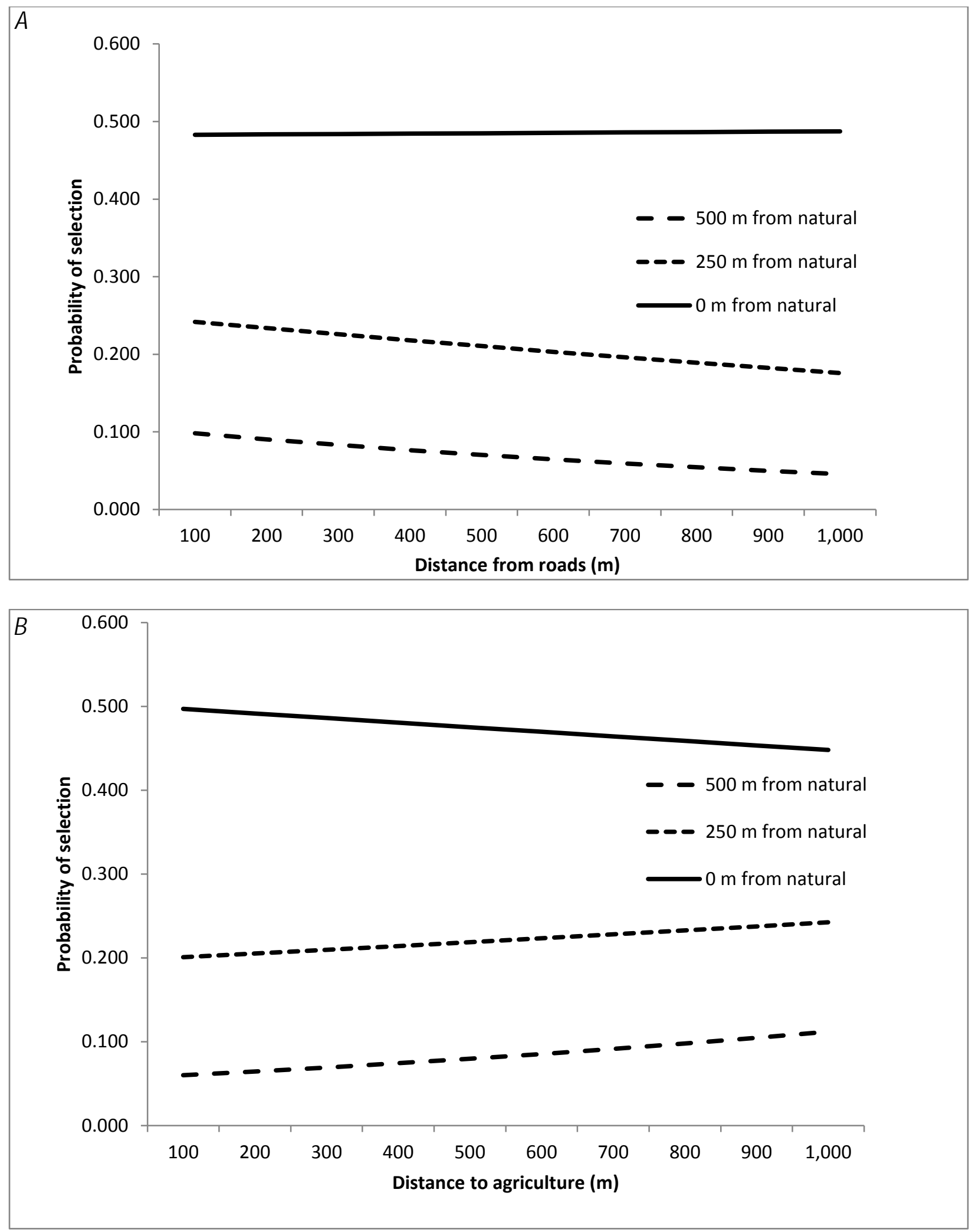

Figure 31. Relation between probability of selection of steps and distance to natural cover by $A$, distance to roads, and $B$, distance to agriculture for female Louisiana black bears during 2010-12 (natural, natural land cover; m, meters). 
For males, the probability of a step increased as the distance to natural land cover and its square at the end of a step decreased and as the distance to agriculture at the end of a step decreased on the basis of the Cox proportional hazards model (table 8). Additionally, we found that step selection was more likely as distance to roads and its square increased. Although the 95\% CI of an interaction effect between distance to natural and agricultural cover types included zero (-2.51-06-3.36-07, table 8), the AIC was much improved, so we kept the interaction term. The deviance reduction associated with the removal of each variable from the model indicates that distance to natural land cover $(-2,779.2)$ had the greatest effect on step selection, followed by distance to roads (-76.5) and distance to agriculture (-4.0). A cutoff of 24 for autocorrelated steps was identified. The pseudo $R^{2}(0.018$, maximum $=0.353)$ and the standardized $\beta$ values indicate that selection strength was generally low for all models (table 8). An interaction was observed between distance to agriculture and season $\left(\beta=-1.48-04, \mathrm{SE}_{\text {robust }}=3.96 \mathrm{E}-05\right)$. On the basis of the mixed effects models, we found that the distance to natural land cover varied by individual bear $\left(\sigma^{2}=5.59 \mathrm{E}-03, P<0.001\right)$, but the relation was expected to be negative for essentially all male bears (>99\%).

When we generated 4,000 dispersal paths, based on the step-selection function models, the least potential interchange was between the TRB and TRC and between UARB and LARB (table 9). The greatest proportion of successful projections was from UARB to TRC (937/4000 and 316/4000 for males and females, respectively), followed by paths in the reverse direction (617/4000 and 199/4000 for males and females, respectively). The cost surface maps revealed patchy values in the Lower Mississippi Alluvial Valley, which is reflective of the fragmented nature of the landscape there (figs. 32 and 33). The addition of 1,000-m- and 3,000-m-wide corridors between the four subpopulations had negligible to slightly negative effects for most pairings, the exception was an increase from the LARB to the UARB for males (table 9). Finally, when only permanently protected natural land cover was considered, the incidence of successful dispersals dramatically decreased (table 9).

Table 9. Number of successful dispersal paths between four black bear subpopulations in Louisiana, 2010-12, with and without the addition of hypothetical corridors and non-permanently protected natural land cover.

[TRB, Tensas River Basin; TRC, Three Rivers Complex; UARB, Upper Atchafalaya River Basin; LARB, Lower Atchafalaya River Basin; km, kilometer; m, meter]

\begin{tabular}{|c|c|c|c|c|}
\hline Dispersal route and distance & $\begin{array}{l}\text { Number of } \\
\text { successful paths } \\
\text { of } 4,000 \text { with no } \\
\text { additional } \\
\text { corridor }\end{array}$ & $\begin{array}{c}\text { Number of } \\
\text { successful paths of } \\
4,000 \text { with addition } \\
\text { of a } 1000-\mathrm{m} \text { wide } \\
\text { corridor }\end{array}$ & $\begin{array}{c}\text { Number of } \\
\text { successful paths of } \\
4,000 \text { with addition } \\
\text { of a } 3000-\mathrm{m} \text { wide } \\
\text { corridor }\end{array}$ & $\begin{array}{l}\text { Number of successful } \\
\text { paths when only } \\
\text { permanently protected } \\
\text { natural land cover } \\
\text { available }\end{array}$ \\
\hline \multicolumn{5}{|l|}{ Females } \\
\hline TRB to TRC (91.3 km) & 0 & 0 & 1 & 0 \\
\hline TRC to TRB (91.3 km) & 1 & 0 & 0 & 0 \\
\hline TRC to UARB (22.8 km) & 199 & 141 & 189 & 0 \\
\hline UARB to TRC (22.8 km) & 316 & 253 & 266 & 1 \\
\hline UARB to LARB (87.1 km) & 0 & 0 & 1 & 0 \\
\hline LARB to UARB $(87.1 \mathrm{~km})$ & 1 & 0 & 0 & 0 \\
\hline \multicolumn{5}{|l|}{ Males } \\
\hline TRB to TRC (91.3 km) & 40 & 32 & 30 & 0 \\
\hline TRC to TRB (91.3 km) & 24 & 24 & 30 & 0 \\
\hline TRC to UARB (22.8 km) & 617 & 541 & 602 & 0 \\
\hline UARB to TRC (22.8 km) & 937 & 879 & 931 & 55 \\
\hline UARB to LARB (87.1 km) & 44 & 32 & 40 & 0 \\
\hline LARB to UARB $(87.1 \mathrm{~km})$ & 56 & 66 & 74 & 0 \\
\hline
\end{tabular}




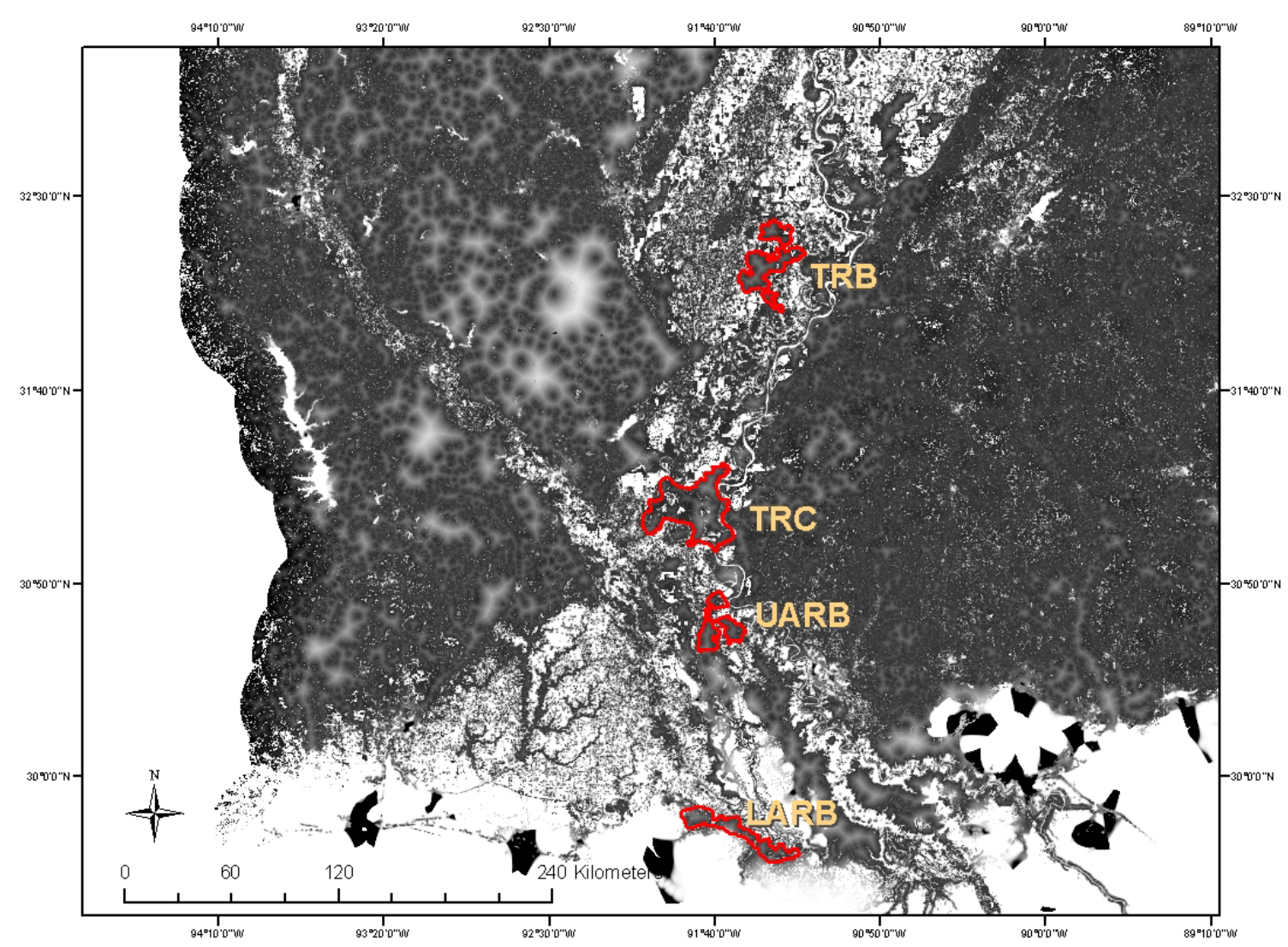

Figure 32. Cost surface for female bears, Louisiana, 2011-12. The red polygons are bear subpopulations; areas with lower costs of travel are in darker colors. (TRB, Tensas River Basin; TRC, Three Rivers Complex; UARB, Upper Atchafalaya River Basin; LARB, Lower Atchafalaya River Basin). 


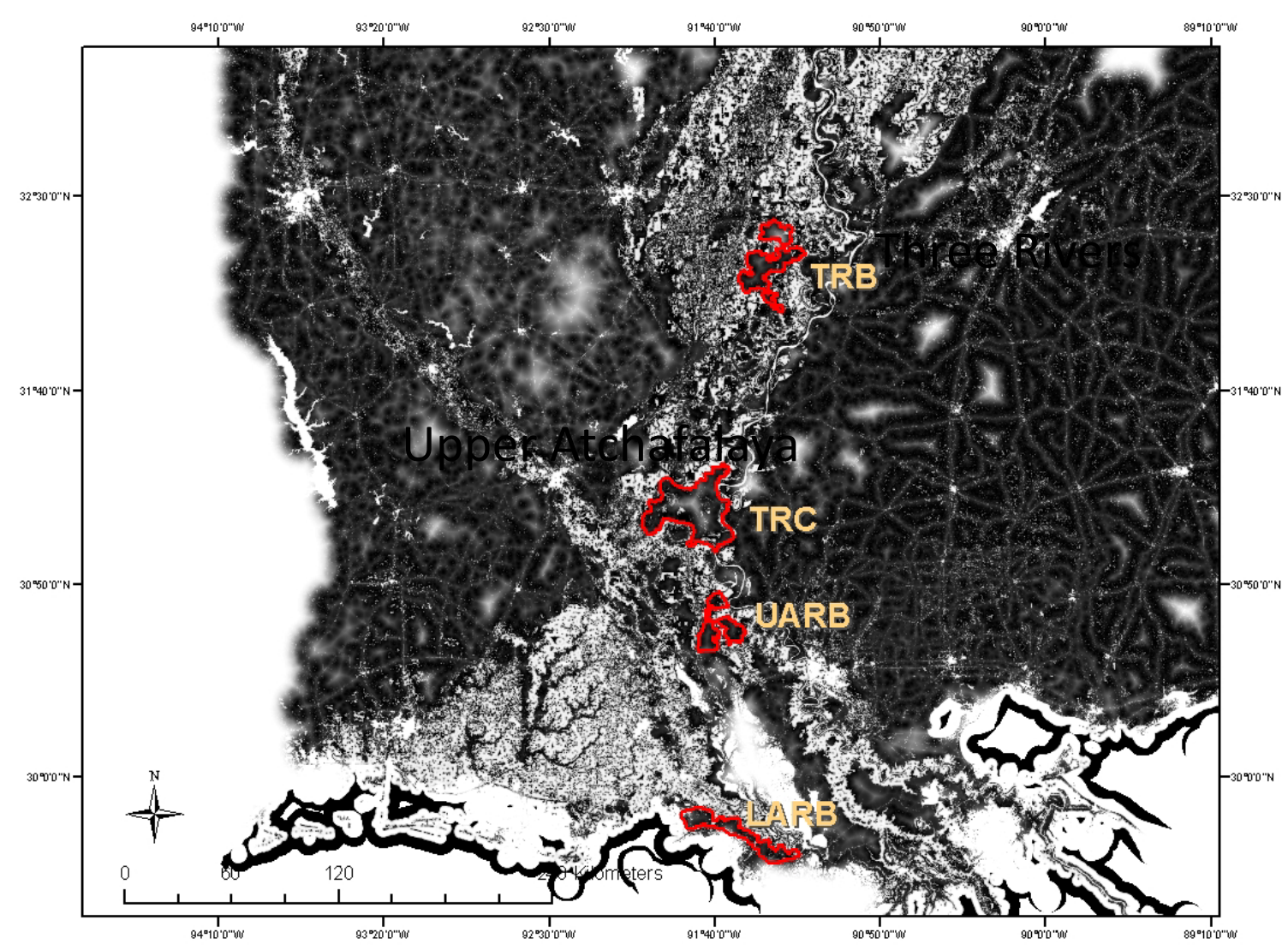

Figure 33. Cost surface for male bears, Louisiana, 2011-12. The red polygons are bear subpopulations; areas with lower costs of travel are in darker colors. (TRB, Tensas River Basin; TRC, Three Rivers Complex; UARB, Upper Atchafalaya River Basin; LARB, Lower Atchafalaya River Basin.

\section{Discussion}

\section{Population Viability Analysis}

\section{Survival Rates of Radio-Collared Adult Female Bears}

Estimates of mean annual adult female survival were slightly lower for the TRC than for the TRB when unknown fates were right censored, and that difference doubled in magnitude when unknown fates were treated as mortalities. We recorded more than twice as many known mortalities in the TRC in only two-thirds of the number of bear-years monitored than in the TRB, indicating mortality risks were greater in the TRC. Although 95\% CI for the two areas overlapped, lower point estimates for the TRC may reflect the effects of additional mortality caused by illegal kills. Nearly half (4 of 9) of the documented mortalities at the TRC were attributed to poaching, and no poaching-related mortalities were documented in the TRB. Annual survival rate estimates for adult females in the TRB and TRC were similar to or slightly greater than survival estimates for other non-hunted black bear populations in the southeastern United States (table 10). Consistent with other studies of adult female survival, the leading cause of mortalities in both study areas was human related ( 3 of 4 in the TRB and 8 of 9 in the TRC). 
Table 10. Estimates of annual survival for adult female black bears from unhunted populations within the southeastern United States.

\begin{tabular}{llc}
\hline \multicolumn{1}{c}{ Source } & \multicolumn{1}{c}{ Location } & Annual survival rate \\
\hline Wear and others 2005 & Felsenthal National Wildlife Refuge & $0.91^{\mathrm{a}}$ \\
Bales and others 2005 & Southeastern Oklahoma & 0.90 \\
Dobey and others 2005 & Osceola National Forest, Florida & 0.97 \\
Clark and Smith 1994 & Interior Highlands, Arkansas & 0.98 \\
Clark and Eastridge 2006 & White River National Wildlife Refuge, Arkansas & $0.98(0.94)^{\mathrm{b}}$ \\
\hline
\end{tabular}

${ }^{\mathrm{a}}$ Survival rate of reintroduced adult females during second year post release.

${ }^{\mathrm{b}}$ Survival rates treating lost signals as censored (first value) or mortalities (inside p).

The prevalence of mortalities of radio-collared adult females caused by poaching in the TRC in this study is in contrast to results from 2001 to 2005 reported by Benson and Chamberlain (2007). Those authors recorded zero illegal kills of 21 reintroduced adult females during the first 5 years of the reintroduction effort in the TRC and concluded that poaching prevalence was lower for TRC than for other bear reintroductions in the southeastern United States. However, we continued data collection following their study and found that all four illegal kills occurred after mid-2006. A potential explanation is that competition for space and resources increased on protected state and Federal lands as population numbers increased, causing some bears to spend more time on less protected private properties where poaching threats may have been greater. However, such range expansion would only account for one-half of the illegal kills of radio-collared females because 2 of 4 occurred on state-owned Wildlife Management Areas.

\section{Reproductive Rates of Radio-Collared Adult Female Bears}

The greater likelihood of females in the TRC to transition between states $\mathrm{C}$ and $\mathrm{Y}$ indicates that cub survival was lower in the TRB. Lower cub survival in the TRB may be caused by greater competition for resources and greater potential for intraspecific killing because that population may be close to carrying capacity. Such density-dependent regulation of dependent offspring survival is a welldocumented aspect of bear population dynamics (Bunnell and Tait, 1981; Clark and Smith, 1994; Czetwertynski and others, 2007). In contrast, females in the TRC were less likely to transition from any of the three reproductive states to state $C$, indicating breeding success was lower in the TRC. Lesser breeding success in the TRC may be related to possible Allee effects caused by few resident breeding males in the area at the onset of the reintroduction project (Courchamp and others, 2008). Despite these differences, stable state probabilities for state $\mathrm{Y}$ for the TRB and TRC were similar, indicating that the positive effect of greater cub survival was largely offset by potential Allee effects.

In general, females in the TRC produced more cubs and more yearlings per litter than females in the TRB. In contrast, $f$ was greater in the TRB, which was primarily caused by the greater proportion of females producing litters of cubs. However, $r$ in the TRC was slightly greater despite that population having a lower proportion of females encumbered by yearlings. The factor driving that difference was that females in the TRC had larger yearling litters, which similar to the reproductive transition analysis, likely reflected greater cub survival in the TRC. 


\section{Demographic Rates from Capture-Mark-Recapture Data}

Annual abundance estimates for the TRB and LARB differed depending on how detection heterogeneity was modeled. In contrast, abundance estimates for the UARB were similar for both models because nearly all females were detected in one or more secondary sampling periods (that is, weeks) of primary sampling periods (that is, years) during which they were alive and present in the study area. Moreover, estimates of $\gamma$ were also affected by model choice because those estimates are linked with estimates of abundance. However, estimates of $\phi$ were similar for all populations regardless of heterogeneity model choice because $\phi$ is more robust to heterogeneity biases (Abadi and others, 2013). Therefore, estimates of $\bar{\lambda}_{G}$ likely were robust for the heterogeneity model because growth and sustainability of bear populations are primarily driven by adult female survival.

Another potential difficulty for DNA-based CMR studies is determining whether all age cohorts within a population are being sampled because age data generally cannot be obtained from DNA. We found that cubs known to be alive and present in the study area were not detected at hair-collection sites. This is in contrast to Kendall and others (2009) who concluded their abundance estimates for grizzly bears (Ursus arctos) included all age cohorts; this difference was likely due to the physiological differences between grizzly and black bear cubs and different wire configurations. Because field collection methods were standardized across all study areas, abundance estimates and demographic rate estimates from CMR analyses for the TRB, UARB, and LARB need to be interpreted as pertaining to age cohorts 1-year old and older.

Although the specific patterns of variation in abundance and recruitment differed between models, estimated $\bar{\lambda}_{G}$ during this study was stable for the TRB, whereas increasing estimated growth was determined for the UARB, possibly because bears at TRB may be at or near carrying capacity. Greater variability of growth rates in the UARB may reflect greater environmental variation in recruitment or greater demographic variability caused by the smaller population size (Shaffer, 1987; White, 2000; Mills, 2007). Apparent survival based on the CMR analysis (0.87-0.93) was much lower than the estimate from the telemetry data 0.97-0.99) at the TRB. That difference was expected because $\phi$ from the CMR analysis includes emigration, whereas survival based on known-fate analysis does not. Apparent survival was slightly lower for the UARB than for the TRB; whether the lower $\phi$ was primarily the result of mortality or permanent emigration is unknown.

The analysis of CMR data for the TRB and UARB showed evidence of a negative relation between per capita recruitment and abundance, which indicates density-dependent regulatory factors. Eberhardt (1977) described an ordered sequence of mechanisms by which large mammal populations are regulated as density approaches carrying capacity. Initially, increased intra-specific competition for resources and direct conspecific-caused mortality would be expected at greater densities and cause reductions in survival of dependent offspring and independent juveniles. As density continues to approach carrying capacity, increases in age of primiparity and decreases in reproductive output would occur. Lastly, survival of adults would be expected to decrease under extreme conditions when population growth drastically overshoots carrying capacity. For bears, several studies have reported evidence supporting such population regulation through an inverse relation between cub survival and population density (Lindzey and others, 1983; Miller and others, 2003; Schwartz and others, 2006; Czetwertynski and others, 2007; Garrison and others, 2007). However, other researchers have reported no or inconclusive evidence that population density affects demographic rates in bears (Elowe and Dodge, 1989; Miller, 1994; Sargeant and Ruff, 2001; Obbard and Howe, 2008). Whether population regulation in bears can be rigorously detected and measured has also been questioned (Derocher and Taylor, 1994; Garshelis, 1994; McLellan, 1994). 
Our analysis demonstrated a negative relation between per capita recruitment and abundance. We estimated per capita recruitment, which is defined as the number of bears new to the population (that is, recruits) divided by the number of resident bears. This definition does not distinguish between in situ recruits that are born in the study area and immigrant recruits that disperse to the study area from adjacent but unsampled areas. However, the analysis was limited to females which typically are poor dispersers and display strong natal site fidelity. Therefore, per capita recruitment most likely reflects true in situ recruitment. Moreover, because the sampled population in the CMR dataset was restricted to bears more than 1 year old, recruitment estimates for this study are to be interpreted as in situ recruitment of yearling bears. That interpretation prohibits a clear understanding of which vital rates were being influenced by population density because the data do not allow separating yearling recruitment into demographic components (that is, female reproductive rate and cub survival). However, multiple mechanisms likely operate simultaneously to regulate populations that are near carrying capacity (Eberhardt, 1977). Such synergistic effects may explain why a density-dependent relation was detected in this study because per capita yearling recruitment represents the cumulative effects of multiple demographic processes that may be regulated by density. Also, the longer time period of this study likely contributed to a greater ability to detect density dependence.

Estimating process variation of demographic rates over time is critical for incorporating temporal stochasticity into population projection models used for population viability assessments (White, 2000). Reliability of variance estimates in terms of bias and precision for CMR analyses is linked to the number of animals sampled within each year and to the number of years of sampling (Burnham and White, 2002; White and others, 2009). Using simulated datasets and Bayesian estimation methods, White and others, (2009) found that estimates of temporal variance for apparent survival generally were positively biased when the number of occasions (for example, years) was seven and estimates were based on the mean of the posterior distribution. Although White and others (2009) did not explicitly discuss reliability of estimates that were based on other measures of central tendency (for example, median or mode), their simulation results based on seven occasions and the posterior mode as the estimator (see table 3 of White and others, 2009) indicated substantial negative bias. We chose to base our estimates of temporal process variances on posterior medians, which typically fall in between the mean and mode of skewed distributions, thereby minimizing potential bias. Moreover, CMR data collection has continued on the TRB and UARB, which will extend the time series and should facilitate more robust estimation of temporal process variation in the future.

We estimated $\gamma$ and $\lambda$ for only two intervals at LARB, and both substantially differed by interval. Whether the large difference across intervals is because population dynamics at LARB truly are more variable or because this study was conducted during an unusual sequence of extreme dynamics cannot be determined without a longer time series of data. Moreover, $\phi$ was considerably lower at the LARB than at the TRB or UARB, which was likely due to greater exposure to anthropogenic causes of mortality compared with that of other Louisiana black bear populations (Pace and others, 2000). Because data for only two annual intervals were collected in the LARB, we were unable to explicitly model and reliably estimate global means and annual variation for vital rates.

Although adult female survival rates (0.93-0.97) at TRC were high and comparable with those of other bear populations in the Southeast (table 10), fecundity for breeding females was relatively low, which contributed to lower $\lambda_{\text {Asym }}$. Whether these low reproductive rates will persist is unknown, but the high stable state probability of barren females (B) at TRC indicates an Allee effect caused by the initially low numbers of adult males there. This situation could change as cubs born at TRC grow older and reach maturity and as more males immigrate from UARB. Other researchers have documented low 
initial growth rates of reintroduced bear populations, which dramatically increased in subsequent years (S. Murphy, University of Kentucky and J. Clark, U.S. Geological Survey, unpub. data, 2014).

\section{Stochastic Population Modeling and Extinction Risk Assessment}

Estimates of $\lambda_{\text {Asym }}$ for the TRB were positive regardless of the adult female survival rate (that is, AC versus $A D)$, whereas CMR-based estimates of $\bar{\lambda}_{G}$ were stable to slightly decreasing. The discrepancy between $\bar{\lambda}_{G}$ and $\lambda_{\text {Asym }}$ is to be expected because $\bar{\lambda}_{G}$ inherently includes temporal stochasticity in vital rates that cause lower overall future growth rates, whereas $\lambda_{\text {Asym }}$ assumes stationary, environmental conditions and a stable age distribution resulting in greater growth rates (Morris and Doak, 2002; Mills, 2007). Therefore, if the true population in the TRB was decreasing at substantially high rate, estimates of $\lambda_{\text {Asym }}$ would likely have been $<1$, which was not the case in this study. Overall, estimates of $\lambda$ at TRB were similar whether projections were based on CMR or telemetry data, which lends some validity to the matrix methods used for the TRC projections. At TRC, only the most optimistic estimate of adult female survival resulted in positive population growth, indicating that population may not yet be self-sustaining.

We did not estimate or account for temporal correlation of vital rates among individual component populations in the projection models. Such correlations are potentially important to metapopulation dynamics because they cause temporal synchrony of individual population dynamics and affect global extinction of the entire population system (Harrison and Quinn, 1989; Heino and others, 1997; Palmqvist and Lundberg, 1998). For example, if populations are located within sufficient proximity such that they are affected by the same environmental variation affecting vital rates, probabilities of persistence will be lower for the entire system than if they are assumed to be independent. That is because a potential decline owing to a stochastic environmental event would be likely to similarly affect all subpopulations, with less chance that one population could compensate for the other. Although the presence of temporal correlations among Louisiana black bear populations would reduce long-term viability of the entire system, the high persistence probabilities estimated for TRB and UARB would negate any co-variation in parameters because the probability that at least one population persists would be at least as great as that for the population with the greater probability of persistence, which was $>95$ percent. Moreover, the viability analysis did not include persistence probabilities for Louisiana black bears in the LARB or in Mississippi. Inclusion of those populations would further increase the likelihood of long-term viability of bears in general for the entire system.

Correlations among population-specific vital rates were not included in the projections because the length of our time series of CMR data was insufficient to reliably estimate covariances among parameters. Such correlations can decrease persistence probabilities for the same general reasons as those among population correlations (Morris and Doak, 2002). However, high means and low variances of adult female survival rates and relatively higher variation in per capita recruitment indicate population dynamics are primarily driven by recruitment processes rather than survival processes, which would dampen potential covariance effects. Nonetheless, CMR-based monitoring efforts in the TRB and UARB are expected to continue (Maria Davidson, LDWF, oral commun., 2014), which should allow estimation of among-parameter covariance and their effects on population dynamics in the future.

Incorporating density-dependence into projection models inherently causes compensatory mechanisms to return populations to equilibrium levels and reduces the overall risk of extinction (Ginzburg and others, 1990). Furthermore, inference about long-term population persistence is sensitive to the specific form of the density-dependent relation included in a projection model used for population viability analysis (Mills and others, 1996). Therefore, density-dependent relations ideally would be 
based on empirical data collected from the population of interest rather than be assumed from population theory or extrapolated from other populations or species. Consequently, we based our density relation on the CMR data for the TRB and UARB. However, parameter estimation uncertainty prevented conclusive determination of the form of density dependence that could result in misleading conclusions about population persistence if that uncertainty was ignored. To account for that uncertainty, our all-uncertainty projections for the TRB and UARB explicitly incorporated parameter uncertainty, including the density-dependence parameter, into the simulations; we conclude that incorporating density dependence into the projection models was justified and that inferences about the long-term persistence of those populations were reliable. Furthermore, results from the projections incorporating all sources of uncertainty represent the most conservative estimates of probabilities of persistence for the TRB and UARB.

For the TRC, where data on density effects were lacking, two different strengths of density dependence were tested. Regulatory mechanisms in large mammals are expected to operate only when populations are near carrying capacity (Eberhardt, 1977, Fowler, 1981) and may not be realistic for a recently re-established population such as the TRC. However, population projection models that do not include regulatory mechanisms would result in exponential growth, given sufficient vital rates, which would also be unrealistic. In contrast, not incorporating a density-dependence relation in demographic rates could eliminate any compensatory response for a small declining population that could mistakenly increase probabilities of extinction. Because simulation results show that long-term persistence is least sensitive to the form of population regulation when compared with the method used to estimate adult female survival and whether only temporal process or all uncertainty in vital rates was included, we conclude that the forms of density dependence we used did not result in misleading inferences about the viability of the TRC.

To our knowledge, this study is the first to perform a risk assessment for determining recovery status for a threatened terrestrial mammal species using Bayesian population viability analysis (PVA). Explicitly incorporating parameter uncertainty through the use of Bayesian posterior distributions is preferred because it results in a wider distribution of extinction times that is more likely to contain the true distribution than use of other methods (Wade, 2002). Moreover, Bayesian PVAs have the added benefit of expressing extinction risk in terms of a frequency-based framework that is more readily incorporated into delisting decisions and adaptive management components of recovery plans (Goodman, 2002). For example, Bayesian PVAs that incorporate multiple sources of variation, including parameter uncertainty and process variation, (that is, temporal and demographic variance) typically result in more conservative estimates of probabilities of persistence, which lowers the chances of wrongly deciding to delist imperiled species.

PVA has often come under scrutiny for its ability to produce reliable risk assessments for the conservation and management of imperiled species. Some common criticisms include misuse of generic software packages to conduct such analyses, lack of sufficient time series data to account for environmental variation, and exclusion or inappropriate estimation of parameter uncertainty (Taylor, 1995; Beissinger and Westphal, 1998). However, Brook and others (2000) evaluated the performance of PVAs by conducting separate PVAs for 21 species for which sufficient data were available, using half the data for parameter estimation and population forecasting and half for model validation. Those authors found that estimates of extinction risk were reliable in most instances.

Regardless of the level of parameter uncertainty that was incorporated for the TRB, the probabilities of persistence were $>95$ percent and were viable, based on the definition stated in the Recovery Plan. For the UARB, all projections, including process-only variation or parameter uncertainty based on the $50 \%$ CI of vital rate posterior distributions, resulted in a $>0.95$-percent chance 
of persistence, whereas simulations based on CMR Model 2 and the 95\% CI of posterior distributions, resulted in estimates of persistence that were less than the viability threshold (0.849). The Recovery Plan states that at least two populations must be viable, one in the Tensas River Basin and one in the Atchafalaya River Basin. Our results indicate that the probability of persistence of the UARB population is $<95 \%$ under the most pessimistic set of assumptions, meaning that the literal translation of criterion 1 was not met. However, if the intent of specifying that two populations should be viable was to ensure the persistence of the overall population of $U$. a. luteolus, then our data indicate that the viability threshold in the Recovery Plan was indeed met because the probability of persistence of the population system was $>95 \%$ under the most pessimistic set of assumptions.

\section{Connectivity Analysis}

\section{Population Structure and Migrant Analysis}

Genetic clustering results from Program STRUCTURE analyses, assuming $K=2$ and using genotype data from all populations in the LMAV and the MINN population, partitioned bears by geographic regions roughly associated with the northern and southern parts of the LMAV with MINN being grouped with the southern bear clade (ancestor and all its descendants). The inclusion of MINN and UARB bears into the same cluster likely reflects the differential effects of the previous restocking effort. Of the 161 bears released from 1964 to 1967, 131 were released in the UARB and 31 were released in the TRB (Taylor, 1971). The greater number of bears released in the UARB likely resulted in more bears establishing home ranges in that area, reproducing, and eventually having a greater influence on the future genetic composition in the UARB than in the TRB. That influence would explain the greater affinity between the UARB and MINN supported by our results.

The inclusion of bears from the LARB into the southern clade is more difficult to explain because no bears were released in the LARB. However, the LARB is located approximately $100 \mathrm{~km}$ from the release site in the UARB. Given that bears were released during the summer without an acclimation period (that is, hard released) and the propensity for hard-released bears to disperse longer distances from release sites (Rogers, 1973; Eastridge and Clark, 2001; Clark and others, 2002), a sufficient number of bears released in the UARB may have dispersed to the LARB. From 1965 to 1969, released bears were reported to have dispersed to Texas, Mississippi, Arkansas, and 37 of the 64 Louisiana parishes (Taylor, 1971). Other researchers (Warrilow and others, 2001; Csiki and others, 2003; Triant and others, 2004) found greater genetic similarity between bears in the UARB and LARB than between either of those areas and the TRB or WRB.

Clustering of bears from the WRB, TRB, and MISS into a single group indicates that those areas were relatively unaffected by the restocking program in Louisiana and retained a greater proportion of their historic genetic composition. Of those 3 areas, the TRB and WRB are the only 2 areas that support extant populations that have never been extirpated, though the TRB population was augmented with bears from MINN. Contiguous bottomland hardwood forests once existed throughout the LMAV that likely supported a continuous bear population between the WRB and TRB. Prior to extensive loss and fragmentation of habitat that led to the isolation of bear populations in the LMAV, a continuous distribution of bears may have facilitated sufficient historic gene flow throughout the region to cause allele frequencies in the WRB to have been correlated with those in the TRB. Although black bears were nearly extirpated ( $\leq 12$ individuals) from Mississippi by the late 1930s, bears from Louisiana, Arkansas, and Alabama have recently recolonized formerly occupied habitat in western and southeastern parts of Mississippi (Simek and others, 2012). Because of the relatively close proximity of 
the WRB and TRB to Mississippi, those populations are the most likely sources of migrants into western Mississippi, which would explain clustering of MISS bears with TRB and WRB bears.

When we increased the number of potential genetic clusters in the Program STRUCTURE analysis from 2 to 3, the LARB was the first population to separate from the other clusters. Genetic differentiation in the LARB from other populations in Louisiana was likely caused by a combination of factors. By the late 1950s, the number of black bears in Louisiana was reduced to only 80-120 individuals in isolated patches of habitat in the LARB and TRB (St. Amant, 1959). Those areas were separated by more than $275 \mathrm{~km}$, which is beyond the typical dispersal distance capabilities of bears and likely resulted in limited historical gene flow between those populations caused by isolation-by-distance effects (Wright, 1943). Moreover, such low numbers may have resulted in rapid genetic drift (Fisher, 1930; Wright, 1931), which may have further contributed to genetic divergence between those populations.

The Program STRUCTURE analysis that assumed 4 population clusters produced the first instance of the TRB and WRB splitting into 2 separate clusters, indicating bears in the TRB had a closer genetic affinity to bears in the WRB than to bears from any of the other extant populations in the LMAV. Additionally, three males in the TRB were identified as migrants from the WRB, which indicates that the greater affinity may in part be the result of contemporary gene flow, in turn indicating that a movement pathway exists between those populations. Indeed, bears could disperse directly from the WRB to the TRB, as evidenced by a male bear with Arkansas ear tags captured in 2005 at Lake Ophelia National Wildlife Refuge in Avoyelles Parish, La. (Maria Davidson, LDWF, unpub. data, 2005), which is $100 \mathrm{~km}$ south of the TRB and almost $300 \mathrm{~km}$ south of the WRB. That bear was identified as a nuisance bear that was captured and released near White River National Wildlife Refuge in the WRB. Although that bear may have bypassed the TRB and did not contribute to gene flow in that population, its presence in WRB and TRB indicates movement directly from the WRB to the TRB is possible.

Alternatively, the migrants we detected may be the result of the reintroduction of bears from the WRB to FNWR. From 2000 to 2002, 23 adult female bears and 56 cubs were translocated from the WRB to FNWR (Wear and others, 2005), which is approximately $100 \mathrm{~km}$ northwest of the TRB. Of the bears moved to FNWR, one radio-collared adult female was known to have subsequently dispersed as far south as the Tensas River National Wildlife Refuge in the TRB (Maria Davidson, LDWF, unpub. data, 2014), demonstrating that dispersal from FNWR to the TRB has occurred. Another potential route by which migration from the WRB to the TRB could have occurred is through Mississippi. Several lines of evidence support this hypothesis. First, we found direct evidence that WRB bears have dispersed across the Mississippi River and recolonized forested habitats in western Mississippi. Second, we documented movement from the TRB to Mississippi on the basis of DNA-based CMR and live-capture data, which indicate that movement in the reverse direction is possible. Third, movement of several radio-collared bears from the TRB into Mississippi has been documented over the past 15 years (19992013; Maria Davidson, LDWF, unpub. data, 2014). Lastly, reproduction has recently been documented in an area of Mississippi located east and northeast of the TRB (Simek and others, 2012). Genetic evidence of the presence of bears with full and partial WRB ancestry in those counties, combined with the documented ability of bears to cross the Mississippi River, indicates dispersal of bears with WRB ancestry into the TRB by way of Mississippi is likely.

At $K=5$, bears in the UARB were first distinguished from MINN bears, which indicates the lasting genetic effects of the reintroduction in the 1960s. Differentiation between those populations also improved inference about admixed cubs in the TRC by identifying the UARB as the source of immigrant sires. Moreover, we found evidence of indirect interchange between the TRB and UARB by 
way of the TRC, which indicates the presence of pathways necessary for such interchange. However, interchange between the TRC and UARB does not appear to be symmetrical because no instances of bears with partial TRB ancestry were found in the UARB that would indicate movement in that direction. Such asymmetrical movement demonstrates that existence of pathways alone is not a sufficient condition for interchange to occur. Although we detected movements of bears only from the TRC to the TRB, we were unable to determine symmetry of movements because we lacked data for potential migrants into the TRC (that is, samples from bears other than translocated females and their offspring).

The only evidence of direct movement (that is, a known individual) among any of the three extant Louisiana black bear populations identified in the Recovery Plan was a single female migrant in the TRB that was identified as coming from the LARB. Given that the TRB is well beyond the typical natural dispersal distance of female black bears from the LARB and that nuisance bears in the LARB are occasionally moved to the northern part of the TRC (Maria Davidson, LDWF, oral commun., 2014), that female most likely was a translocated nuisance bear that found its way to TRB.

One male detected at hair-collection sites in the TRB was classified as being a second generation migrant from the UARB. Whether this bear is a resident offspring of a first generation UARB migrant to the TRB or is mixed ancestry offspring born in the TRC that subsequently dispersed to the TRB could not be determined. However, given the high proportion of cubs born in the TRC with mixed ancestry and documented dispersal of young males from the TRC to the TRB, that bear most likely was a cub produced in the TRC by a female with TRB ancestry and male with UARB ancestry that dispersed. Again, that bear indicates gene flow from the UARB to the TRB that was likely facilitated by the presence of the reintroduced population in the TRC.

Although analyses that assumed clusters of bears $>5$ were not well supported by the data, an interesting pattern within the TRB was apparent. At $K=6$, bears in the TRB were separated into two primary groups. When individuals in those two groups were plotted, the observed differentiation coarsely aligned with Interstate 20 (I-20) transportation corridor (fig 26), which indicates a restriction of gene flow may have occurred at some point in the past between bears north of those roadways and bears to the south. Whether or not this pattern was caused by historical fragmentation, a contemporary restriction in gene flow, or random chance could not be determined. Given the relatively long generation time of black bears, the time since construction of I-20 in the 1950s may not have been sufficient to have produced conclusive evidence of restricted gene flow. Regardless, relatively high rates of mortality associated with vehicle collisions along a 30-km section of the I-20 corridor (14 mortalities from 201013; Maria Davidson, LDWF, unpub. data, 2014) indicate those highways negatively affect successful movement.

Collective results from our clustering analyses indicate that the three subpopulations of Louisiana black bears identified in the Recovery Plan (U.S. Fish and Wildlife Service, 1995) are genetically distinct from each other. Moreover, bears in those populations show significant genetic dissimilarities when compared with bears from the WRB and MINN. Identifying the factors causing the genetic structuring of those populations is a difficult and complex problem because individual populations were affected by varying levels of many different factors. However, differentiation among populations within the LMAV can be reduced to three main factors-(1) restricted gene flow between populations caused by extensive loss and fragmentation of habitat, (2) accelerated genetic drift related to past reductions in local population abundances, and (3) differing levels of genetic introgression that resulted from the historic reintroduction of bears from Minnesota into Louisiana. Fortunately, our results also revealed evidence that gene flow has resumed among some populations, facilitated by the reintroduction efforts at the TRC and perhaps FNWR. 
Although bears in Louisiana may have affinities to MINN bears and WRB bears may have migrated to TRB, the level of genetic affinity or differentiation between populations is not sufficient evidence for determining taxonomic status (Allendorf and others, 2013) and thus ideally would not be the only measure used to warrant protection. Moreover, the issue of true taxonomic status may be irrelevant from a legal standpoint because American black bears are indistinguishable from Louisiana black bears on the basis of physical characteristics and are afforded protection within the historic range of the Louisiana black bear under the similarity of appearance section of the ESA (U.S. Fish and Wildlife Service, 1992). The data from this study indicate a genetic interchange by bears from outside the range of $U$. a. luteolus (that is, Arkansas) with bears in Louisiana and Mississippi. Given the historical proximity of WRB bears, that ingress probably needs to be considered a positive genetic and demographic contribution to the Louisiana black bear, regardless of taxonomic delineation.

\section{GPS Telemetry Data to Estimate Successful Interchange Rates and Paths}

Connectivity ideally is measured from a functional perspective rather than judged only on the basis of spatial arrangement of habitat. The movement characteristics of the focal species, such as its dispersal capabilities and behavioral responses to the physical structure of the landscape, also need to be taken into account. The projection and estimation of successful interchange events on the basis of stepselection function was an attempt to link those concepts in a single analysis. Results of the stepselection models used in this study indicate that the relative potential for natural interchange between UARB and TRC is high, which was supported by the genetic data. No evidence of natural female dispersal between subpopulations has been documented thus far, which is also consistent with model predictions. The genetic data also support these results as the factorial correspondence analysis indicated that TRC bears were clustered between the TRB (where they originated) and the UARB. About half the sampled cubs had mixed ancestry between TRB and UARB, which suggests that males dispersed from UARB and bred with females at TRC. The step-selection model predicted that dispersals between the LARB and UARB populations were infrequent but possible for males but nearly nonexistent for females. Genetic structure was high between UARB and LARB bears, with no migrants being identified in either. All of the known long-distance dispersals between our study areas were by males. The higher number of successful interchanges from the UARB to TRC compared with dispersals in the opposite direction may have been partially because of the larger relative size of the TRC, thus making intersections more likely.

The factor having greatest influence on successful interchange probably was Euclidean distance (figs. 34-35). The distance between the TRB and TRC (91.3 km) and between the LARB and UARB $(87.1 \mathrm{~km})$ is so great that females likely would not move enough within a year and male movements of that length would be infrequent. The reintroduced population at TRC, however, essentially reduced the distance between occupied fragments and resulted in increased estimated interchange rates. Without that stepping-stone population, our model predicted no dispersals of either sex from UARB to TRB. The use of land cover as stepping stones for movement also was documented for grizzly bears (Proctor and others, 2004).

The hypothetical corridors were effective for linking subpopulations only for males dispersing from the LARB to UARB. When viewing the projected pathways relative to the natural cover type, it is evident that our model projected male and female bears occasionally crossing gaps, even some large ones, if the step ended in or near natural cover (fig. 36). We speculate that the hypothetical corridors were not more effective than the broken habitat matrix that surrounded many of the subpopulations. Furthermore, the hypothetical corridors may have led bears into areas where the surrounding matrix of natural cover was sparse. This is not to say that corridors are not important. The increase in movements 
from the LARB to the UARB indicates that corridors through a depauperate natural land cover matrix can be beneficial.

Only one radio-collared bear (Nstar13, displacement $=88.5 \mathrm{~km}$ ) exhibited the type of longrange, directed dispersal behavior that has been documented in other black bears studies (80-145 km; Rogers, 1987; Lee and Vaughan, 2003); most other males did not $(\bar{x}=15.0 \mathrm{~km}$, maximum $=36.8 \mathrm{~km})$. It should be noted that the number of pathways projected for each subpopulation $(4,000)$ greatly exceeds the number of likely dispersal events given the small number of bears in the four subpopulations and thus should not be interpreted as the number of expected migrants between subpopulation pairs. No dispersal events for females, which are rare, were recorded, and females tend to disperse only as a result of extreme environmental events (Hellgren and others, 2005). Factors that would account for home range fidelity were not included in the model (Rhodes and others, 2005) because the goal was to characterize the type of movement activity that might be associated with an animal in unfamiliar surroundings in search of suitable habitat. Nevertheless, our model predictions generally agreed with our genetic and trapping data. 


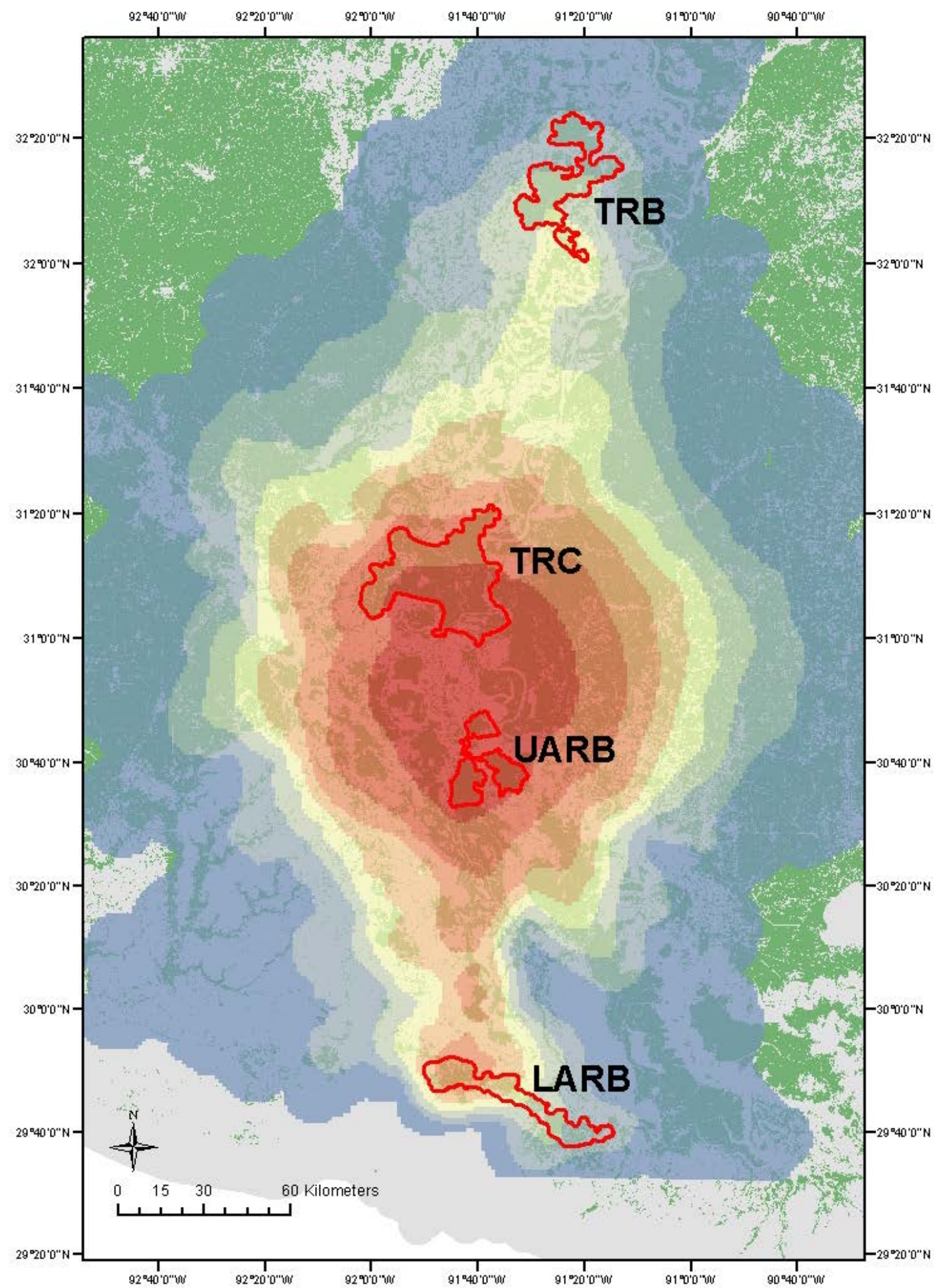

Figure 34. Relative densities of simulated successful dispersal trajectories for male bears in Louisiana. Red polygons are the four Louisiana black bear subpopulations. Highest probabilities of use are in red and lowest are in blue. (TRB, Tensas River Basin; TRC, Three Rivers Complex; UARB, Upper Atchafalaya River Basin; LARB, Lower Atchafalaya River Basin). 


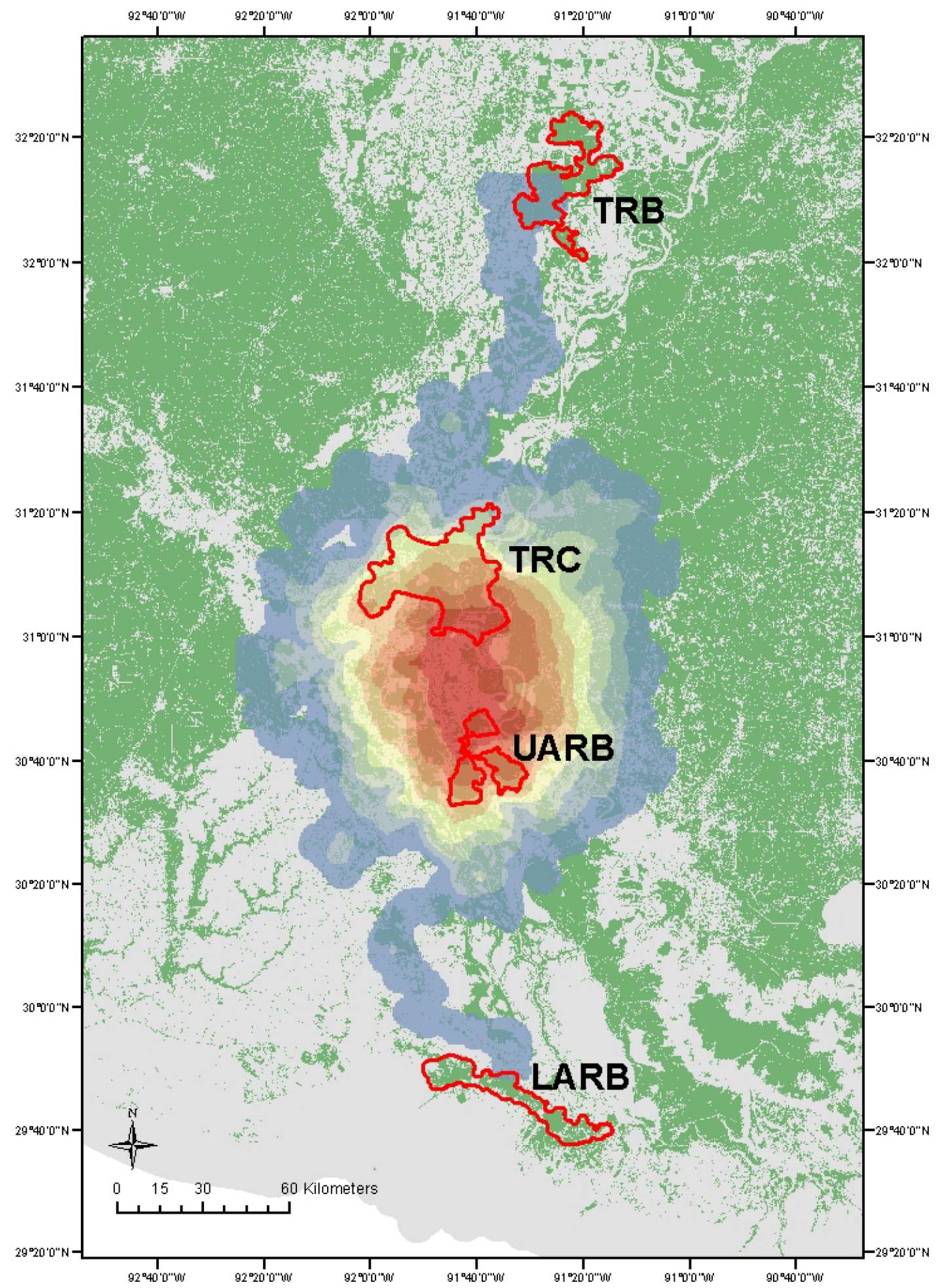

Figure 35. Relative densities of simulated successful dispersal trajectories for female bears in Louisiana. Red polygons are the four Louisiana black bear subpopulations. Highest probabilities of use are in red and lowest are in blue.(TRB, Tensas River Basin; TRC, Three Rivers Complex; UARB, Upper Atchafalaya River Basin; LARB, Lower Atchafalaya River Basin. 


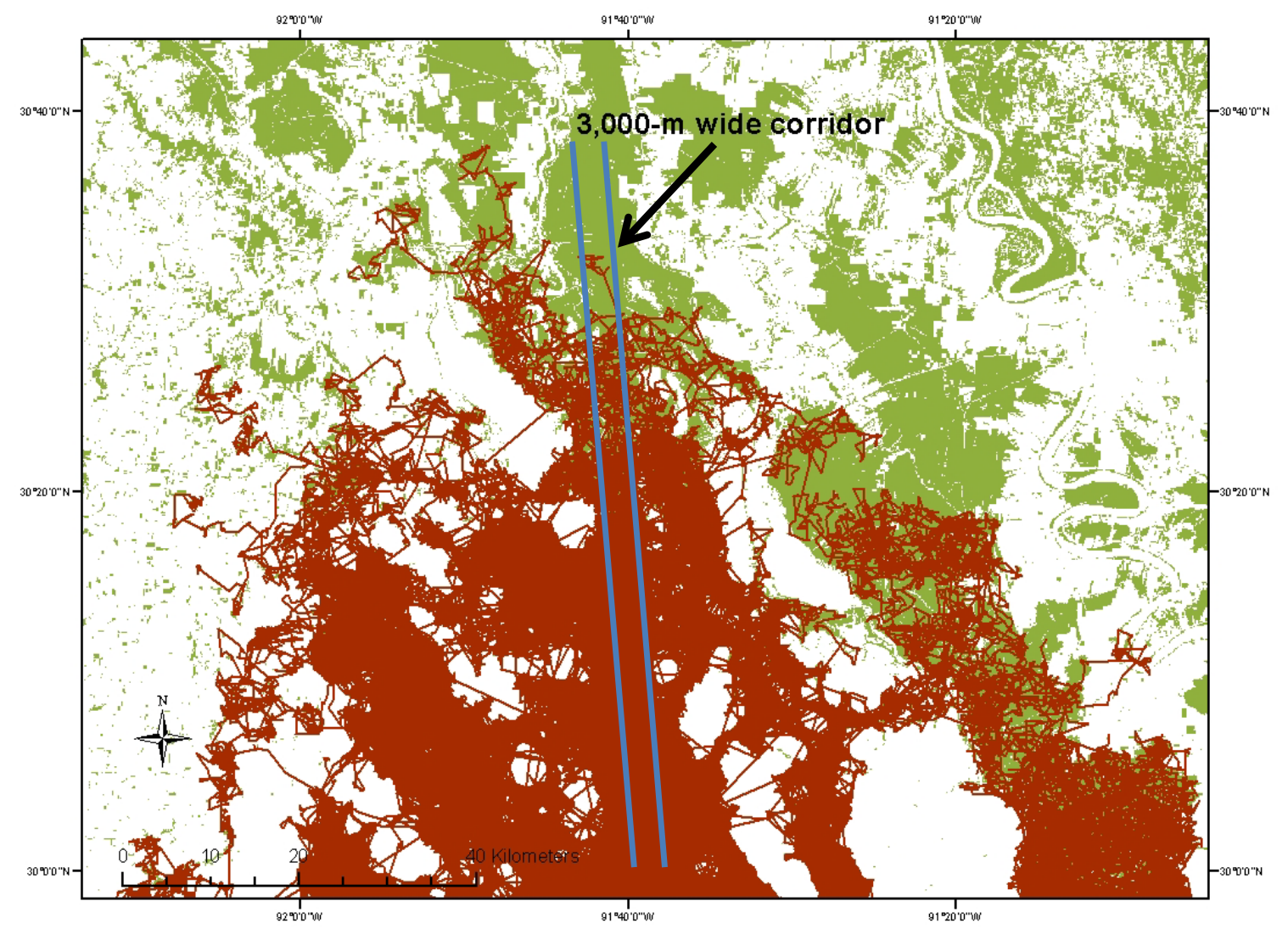

Figure 36. Projected movement paths for female Louisiana black bears (red) after the addition of a hypothetical 3,000-meter-(m)-wide corridor between the Lower Atchafalaya River Basin and Upper Atchafalaya River Basin subpopulations in Louisiana. Natural cover types are in green and non-natural are in white.

Selection was relatively weak for most variables, based on standardized $\beta$ values and the low pseudo- $R^{2}$, indicating that there was much variation in step selection that cannot be explained. There may be environmental variables that were not measured or included in the model that would have had better explanatory power. Also, we did not model the step selection as a function of time of day or month because the goal was to project movement pathways over the course of one active season that would encompass all times of day and most months. Furthermore, the study did not investigate whether presence of conspecifics affected movement paths or dispersal behavior. We assumed there were no bears between subpopulations. Therefore, the estimates are only approximations of the probabilities of successful movement between subpopulations over a 1-year activity season. We were interested in dispersal movement, however, and conclude it was appropriate to model only landscape attributes.

Forester and others (2009) explored biases associated with step-selection functions that were based on case-control sampling and found that $\beta$ estimates can be biased if selection depends on step length. Step length was not added as a covariate for the step-selection models because of the greatly increased complexity in projecting movement paths. However, Forester and others (2009) found that such bias was minimal with empirical sampling of step lengths and turning angles when selection levels were low. Consequently, the addition of that covariate may not have been necessary in this case, given the generally low relative strength of selection exhibited by the models used in this study.

Landscape ecologists have used genetics data to develop maps that show resistance to migration or gene flow on the basis of environmental barriers (Proctor and others, 2005; Cushman and others, 
2006; Storfer and others, 2010). However, the biggest challenge is assigning resistance values to individual landscape features because the actual effects on movement, survival, and abundance are usually not known (Spear and others, 2010). Least cost pathways are often used to characterize resistance, but such models do not incorporate the stepwise process by which most animals explore new environments and often require that a minimum corridor width be identified, which is difficult to estimate. Our step selection model explicitly incorporated such spatial scaling so a minimum width (or no width at all) is not necessary. We feel that our projected paths are reasonably representative of bear movement across the landscape and conclude that our model is a useful tool for evaluating relative probabilities of successful movement between subpopulations and the potential effects of conservation planning on bear movement.

The 1995 Louisiana black bear Recovery Plan requires the establishment of immigration and emigration corridors between the two viable subpopulations in the Tensas and Atchafalaya River Basins that are considered sustainable (U.S. Fish and Wildlife Service, 1995). Corridors are often touted as effective tools for connecting fragmented landscapes and enabling demographic and genetic interchange between isolated populations (Nelson and others, 2003; Noss, 2003; Dixon and others, 2006). That undoubtedly was the intent when recovery criterion 2 was developed. The projected pathways, CMR data, and genetic analysis indicate that bears from the UARB dispersed to the TRC and bred with reintroduced bears there. The hair-sampling data indicate that some subadult males have dispersed from the TRC to the TRB. Therefore, habitat is present through which contemporary interchange of bears between the Tensas and Atchafalaya River Basins has occurred, though probably not as a result of a continuous corridor of natural cover. Results of the study indicate that the patchwork of natural land cover between subpopulations may be sufficient to allow exchange, at least for males. Mills and Allendorf (1996) recommended 1-10 migrants per generation to avoid the loss of polymorphism and heterozygosity in subpopulations. Current migration rates of males, possibly facilitated by management trapping and relocation of nuisance bears, may be sufficient to avoid inbreeding. For females, dispersal potential was low to non-existent between all subpopulations, and that estimate may be biased high given the propensity of females to remain within their natal home ranges. The addition of contiguous corridors was only marginally effective at increasing successful interchanges and, depending on the habitat context, may reduce them. More important to interchange between the Tensas and Atchafalaya River Basins was the establishment of a reintroduced population at TRC. Without the TRC bears, our model predicted no male interchange between UARB and TRB. For female interchange and demographic rescue to be effective, some habitat between subpopulations probably would have to be permanently occupied. Thus, the establishment of satellite populations of resident breeding bears between the subpopulations to be linked, as illustrated by the TRC population, may be a more effective measure than the establishment of long corridors without a population presence in between.

Finally, criterion 3 of the Recovery Plan requires long-term protection of the habitat and interconnecting corridors that support each of the two viable subpopulations used as justification for delisting. The bear population at TRB exists almost entirely on a National Wildlife Refuge and state lands. Thus, habitat for that subpopulation is presumably protected. At UARB, most of the bears live within the Morganza Spillway, which is under permanent easement by the U.S. Army Corps of Engineers. The other bear subpopulations in Louisiana live on a combination of state, Federal, and private lands. The USFWS has designated 483,932 hectares (ha) as critical habitat for black bears under Section 4 of the Endangered Species Act of 1973. Of the total area, 50,122 ha and 78,588 ha of critical habitat are under Federal and state ownership, respectively (U.S. Fish and Wildlife, 2009), and the bulk of the land is under local or private ownership (355,221 ha). However, the critical habitat designation would be rescinded if delisting occurs. The long-term viability of the TRB bear subpopulation is 
probably assured, given that it is almost entirely within the National Wildlife Refuge. The same may be the case for the UARB subpopulation, given the permanent easements for habitat occupied by most of those bears. However, the Morganza Spillway is prone to catastrophic flooding, and O'Connell and others (2014) report that repeated bouts of inundation could negatively affect those bears. Land occupied by the LARB and TRC subpopulations and most of the habitat matrix that connects all the subpopulations are not in state or Federal ownership. Furthermore, our model indicated that the loss of private lands would result in nearly complete isolation of the four subpopulations. When nonpermanently protected natural cover was removed from the matrix, the incidence of successful interchange declined drastically for both sexes. Thus, private landowners will play an important role in the future of the Louisiana black bear. Conservation easements and other incentives to landowners to keep land in natural cover will be essential.

\section{Summary and Conclusions}

In 1992, the USFWS granted the Ursus americanus luteolus (Louisiana black bear) threatened status under the U.S. Endangered Species Act of 1973, listing loss and fragmentation of habitat as the primary threats (U.S. Fish and Wildlife Service, 1992). The 1995 Recovery Plan outlines recovery goals designed to meet the objective of reducing threats to the Louisiana black bear metapopulation and the habitat supporting it (U.S. Fish and Wildlife Service, 1995). To meet that objective, the Recovery Plan lists the following criteria for delisting:

1. at least 2 viable subpopulations, 1 each in the Tensas and Atchafalaya River Basins;

2. establishment of immigration and emigration corridors between the 2 viable subpopulations; and

3. long-term protection of the habitat and interconnecting corridors that support each of the 2 viable subpopulations used as justification for delisting.

To address those criteria, a study was developed by the U.S. Geological Survey in cooperation with the University of Tennessee, the Louisiana Department of Wildlife and Fisheries, and the U.S. Fish and Wildlife Service. The objectives of the study were to estimate demographic rates and genetic structure of Louisiana black bear populations; evaluate relations between environmental and anthropogenic factors and demographic, genetic, and movement characteristics of Louisiana black bear populations; develop data-driven stochastic population projection models to assess long-term persistence of individual populations and the black bear metapopulation in Louisiana; and determine the manner in which different assumptions about projection model structure and parameter values affect population trajectories and long-term persistence.

Data were collected with non-invasive DNA sampling, live capture, winter den visits, and radio monitoring from 2002 to 2012 in the four areas supporting breeding populations in Louisiana: Tensas River Basin (TRB), Upper Atchafalaya River Basin (UARB), Lower Atchafalaya River Basin (LARB), and Three Rivers Complex (TRC). Bears were live trapped and radio collared in the TRB and TRC to estimate survival and reproductive rates, and matrix models were used to estimate growth rate and viability. DNA was extracted from hair collected at baited, barbed-wire enclosures in TRB, UARB, and LARB to determine individual identities for capture-mark-recapture (CMR) analysis. CMR analysis was used to estimate apparent survival $(\phi)$, per capita recruitment $(\gamma)$, abundance $(N)$, growth rate $(\lambda)$, and long-term viability, based on Bayesian hierarchical modeling methods, to estimate temporal process variance and parameter uncertainty.

From 2002 to 2012, we radio monitored 86 adult females greater than (>) 2 years old within the TRB, representing 305 bear years, and 43 adult females were monitored in the TRC, representing 208 bear years. The mean annual survival rate estimate for TRB ranged from 0.97 (95-percent [\%] credible 
interval $[\mathrm{CI}]=0.93-0.99)$ to $0.99(95 \% \mathrm{CI}=0.96-1.00)$ and for $\mathrm{TRC}$ ranged from $0.93(95 \% \mathrm{CI}=$ 0.85-0.97) to 0.97 (95\% CI = 0.91-0.99). From 2003 to 2013, 130 cub litters for 74 females were observed in TRB, and 74 cub litters for 45 females were observed in TRC. During the same period, 43 yearling litters were observed for 33 females in TRB and 21 yearling litters for 19 females in TRC. Mean cub and yearling litter size were 1.85 and 1.40, respectively, in the TRB and 2.15 and 1.84 in the TRC. Fecundity and yearling recruitment in the TRB were 0.47 and 0.15 , respectively, whereas estimates for the TRC were 0.37 and 0.18 . On the basis of matrix projection models, asymptotic growth rates for the TRC ranged from 0.99 to 1.02, depending on how unresolved fates were treated.

From 2006 to 2012, 23,312 hair samples were collected in the TRB. The weekly number of sites that produced 1or more viable hair samples ranged from 35 to 174, and the weekly number of samples collected ranged from 98 to 1,382. Estimates of abundance $(N)$ for females in TRB ranged from 140 to 163 during 2006-12 when detection heterogeneity was assumed to follow a logistic-normal distribution (Model 1) and from 133 to 158, assuming a 2-point finite mixture distribution (Model 2). Annual estimates of per capital recruitment $\gamma$ ranged from 0.00 to 0.22 , and estimates of apparent survival $(\phi)$ ranged from 0.87 to 0.93 during that period. The geometric mean of $\lambda$ range from 0.97 (annual range $=$ 0.88-1.06) to 1.02 (annual range $=0.98-1.09$ ), depending on model assumptions.

In the UARB, 15 to 607 hair samples were collected each week, and 11,643 samples were collected across all years from 2007 to 2012. In the UARB, estimates of $N$ for females ranged from 25 to 44 during the study period, regardless of heterogeneity model. Estimated $\gamma$ ranged from 0.00 to 0.41 , and $\phi$ ranged from 0.88 to 0.90 during that period. The geometric mean of growth rate $(\lambda)$ ranged from 1.08 (annual range $=0.93-1.29$ ) to 1.09 (annual range $=0.90-1.35$ ), depending on model assumptions.

Estimated $N$ for females in LARB ranged from 78 to 97 from 2010 to 2012, based on Model 1, and ranged from 68 to 84, based on Model 2. Estimates of $\gamma$ were 0.00 (95\% CI $=0-0.03$ ) for 2010-11 and ranged from $0.24(95 \% \mathrm{CI}=0.10-0.50)$ to $0.31(95 \% \mathrm{CI}=0.16-0.51)$ for $2011-12$, depending on the method. We estimated $\phi$ as 0.81 (95\% CI $=0.68-0.90)$ for 2010-11, and from 0.84 (95\% CI $=0.69-0.97)$ to 0.85 (95\% CI $=0.70-0.94)$ for 2011-12, depending on model assumptions. The estimate of $\lambda$ for 2010-11 was 0.81 (95\% CI $=0.68-0.91)$ and for 2011-12 ranged from 1.08 (95\% CI $=0.89-1.37)$ to $1.16(95 \% \mathrm{CI}=0.93-1.41)$, depending on the model.

On the basis of vital rate estimates from Model 1 of the CMR analysis, probability of persistence over 100 years for the TRB population was $>0.999,0.975$, and 0.958 for process-only, $50 \% \mathrm{CI}$, and 95\% CI projections, respectively. Similarly, the probability of persistence, based on Model 2, was $>0.999$, 0.982, 0.958 for process-only, 50\% CI, and 95\% CI projections, respectively. For the UARB, probabilities of persistence based on Model 1 were $>0.999,0.971$, and 0.958 for process-only, $50 \%$ CI, and $95 \%$ CI projections, respectively, and 0.993, 0.929 and 0.849 for Model 2. Using the telemetry and reproductive data from the TRC, probabilities of persistence ranged from 0.295 to 0.999 depending on carrying capacity, the strength of the density dependence, level of uncertainty, and treatment of unresolved signals. Assuming dynamics of the TRB, TRC, and UARB populations were independent and using the most pessimistic population-specific persistence probabilities (that is, 0.958, 0.295, and 0.849, respectively), the overall probability of persistence for bears in that population system was 0.996.

Genetic methods were used to estimate interchange and structure between subpopulations in Louisiana and from Minnesota (MINN, the source for a historic reintroduction effort); Mississippi (MISS); and the White River Basin, Arkansas (WRB). Results of clustering from the factorial correspondence analysis (FCA) indicate various levels of genetic structure among different pairs of study populations. When bears from only the TRB, TRC (cubs only), and UARB were considered, the TRB and UARB populations appeared as substantially distinct genetic groups, whereas cubs born in the TRC were divided between individuals clustering with the TRB and those whose genotypes clustered 
mid-way between the TRB and UARB. Sufficient genetic structure appeared to exist between bears at the WRB and TRB such that recent migrants could be identified. Taken together, results from the allpopulation and the WRB-TRB clustering analyses indicate at least five genetically distinct populations were represented in the genetic data. Within the TRB, the FCA revealed a low level of structure between bears sampled north of Interstate 20 and bears sampled south of Interstate 20. The FCA also revealed evidence of genetic structure in the LARB that corresponded to an eastern cluster and a western cluster with State Highway 317 and adjacent poor quality habitat operating as a potential movement barrier.

On the basis of results from the FCA and Program STRUCTURE analysis, evidence was found of bears in the TRB with partial or entire ancestry assigned to the WRB, UARB, or LARB. Thirty-two bears from northwestern MISS had ancestry mostly associated with the WRB, 10 showed mostly TRB ancestry, and 6 had mixed ancestry between the WRB and TRB. Cubs with mixed ancestry between the TRB and UARB were identified in the TRC and in west-central MISS east of the TRC. The search for migrants using Program GeneClass and Program STRUCTURE identified 3 first-generation migrants from the WRB to the TRB and 1 from the LARB to the TRB. However, the migrant from the LARB was female and unlikely to have naturally dispersed such a large distance. Searches of DNA-based CMR histories and live-capture records identified 3 males in the TRB that dispersed from the TRC, 2 males that dispersed from the UARB to the TRC, 3 females that dispersed from the WRB to MISS, and 1 male that dispersed from the TRB to MISS. Taken together, results of the genetic clustering and migrant analyses combined with capture data provided direct evidence that interchange has occurred from the WRB to the TRB and MISS, from the TRB to MISS, from the UARB to the TRC, and from the TRC to the TRB. Indirect evidence that interchange occurred from the UARB to the TRC and from the UARB to the TRB by way of the TRC was documented. No evidence was found of interchange from any of the subpopulations to the WRB, UARB, or LARB.

Finally, data collected from global positioning system (GPS) radio-collared bears were used to perform a step-selection function analysis to estimate movement paths and interchange rates. From April 2010 to April 2012, global positioning system (GPS) radio collars were placed on 8 female and 23 male bears ranging from 1 to 11 years of age. We obtained 5,400 GPS fixes for females and 30,832 for males from April 2010 to October 2012. Path lengths during the period of monitoring (that is, 25.2 to 804.8 days, $\bar{x}=241.4)$ for females ranged from 32.9 to 419.1 kilometers $(\mathrm{km})(\bar{x}=185.5)$ and for males from 73.5 to $2,670.2 \mathrm{~km}(\bar{x}=623.7)$. For both males and females, the probability of a step being selected increased as the distance to natural land cover and agriculture at the end of the step decreased, and as distance from roads at the end of a step increased. When 4,000 correlated random walks were generated on the basis of the step-selection functions, the least potential interchange was between TRB and TRC and between UARB and LARB. The greatest proportion of successful projections was from UARB to TRC (937/4000 and 316/4000 for males and females, respectively), followed by paths in the reverse direction (617/4000 and 199/4000 for males and females, respectively). Results of the stepselection models indicate that the relative potential for natural interchange between UARB and TRC is high. The genetic data support these results as many TRC cubs were clustered between TRB (where their mothers originated) and UARB (the likely source population of their fathers). Moreover, the genetic data revealed movement of males from TRC to TRB. In contrast, genetic structure was high between UARB and LARB bears with no migrants being identified in either population. The stepselection model predicted that dispersals between the LARB and UARB populations were infrequent but possible for males and nearly nonexistent for females. No evidence of natural female dispersal between subpopulations has been uncovered thus far, which is also consistent with model predictions. 
The goal of this study was to address the three recovery criteria in the 1995 Recovery Plan and to go beyond that to use the best available science to assess long-term viability of the assemblage of bear subpopulations within the historic range of the Louisiana black bear. Those projections are based on the assumption that the environmental and demographic mechanisms affecting population dynamics during the study remain the same for the next 100 years and on assumptions built into the population projection models themselves. The inclusion of covariance among vital rates and populations, the exclusion of density effects, and any number of other modeling choices could change that. However, an attempt was made to take a conservative (pessimistic) approach, and the projections seem to be reasonable and defendable. The bear populations in the LARB and in MISS will only further enhance prospects for overall metapopulation persistence.

Whether the recovery criteria established in the 1995 Recovery Plan have been achieved will largely depend on the assumptions of the projections deemed most reasonable, interpretations of the Recovery Plan, and the levels of uncertainty that authorities determine are acceptable. Our goal was simply to provide the best information possible to state and Federal authorities so that they may make informed choices based on the data regarding whether the individual populations are viable (criterion 1), whether connectivity is established (criterion 2), and whether habitat is adequately protected (criterion 3). Regardless, monitoring for these bear subpopulations should continue for indications of changes in demographics and to collect more data for estimating temporal process variance. We suggest an active adaptive management approach for Louisiana black bears whereby alternative ways to meet management objectives are explored, the outcomes of alternatives based on the current state of knowledge are predicted, one or more of these alternatives are selected, impacts of management actions are monitored, and knowledge is updated and management actions adjusted.

\section{References Cited}

Abadi, F., Botha, A., and Altwegg, R., 2013, Revisiting the effect of capture heterogeneity on survival estimates in capture-mark-recapture studies: does it matter?: PLOS One v. 8, no. 4, e62636.

Allendorf, F.W., Luikart, G.H., and Aitken, S.N., 2013, Conservation and the genetics of populations: West Sussex, UK, John Wiley \& Sons, 610 p.

Anderson, D.R., 1997, Corridor use, feeding ecology, and habitat relationships of black bears in a fragmented landscape in Louisiana: Knoxville, Tenn., University of Tennessee, Master of Science Thesis, $124 \mathrm{p}$.

Anderson, G.S., and Danielson, B.J., 1997, The effects of landscape composition and physiognomy on metapopulation size: the role of corridors: Landscape Ecology, v. 12, p. 261-271.

Arnason, A.N., 1972, Parameter estimates from mark-recapture experiments on two populations subject to migration and death: Researches on Population Ecology (Tokyo), v. 13, p. 97-113.

Bailey, R.G., 1995, Description of the ecoregions of the United States (2d ed. rev. and expanded; 1 st ed. 1980): Washington, D.C., USDA Forest Service Miscellaneous Publication 1391 (rev.), 108 p. with separate map at scale 1:7,500,000.

Bales, S.L., Hellgren, E.C., Leslie, D.M., Jr., and Hemphill, Jr., J., 2005, Dynamics of a recolonizing population of black bears in the Ouachita Mountains of Oklahoma: Wildlife Society Bulletin, v. 33, p. 1342-1351.

Beausoleil, R.A., 1999, Population and spatial ecology of the Louisiana black bear in a fragmented bottomland hardwood forest: Knoxville, Tenn., University of Tennessee, Master of Science Thesis, $122 \mathrm{p}$. 
Beissinger, S.R., and Westphal, M.I., 1998, On the use of demographic models of population viability in endagnered species management: Journal of Wildlife Management, v. 62, p. 821-841.

Belkhir, K., 2004, GENETIX, v.4.05.2, Laboratoire Génome, Populations, Interactions, Adaptation: Montpelier, France, CNRS UPR, Université Montpelier [In French].

Benson, J.F., 2005, Ecology and conservation of Louisiana black bears in the Tensas River Basin and reintroduced populations: Baton Rouge, La., Louisiana State University, Master of Science Thesis, $114 \mathrm{p}$.

Benson, J.F., and Chamberlain, M.J., 2007. Space use and habitat selection by female Louisiana black bears in the Tensas River Basin of Louisiana: Journal of Wildlife Management, v. 71, p. 117-126.

Besbeas, P.J., Freeman, S.N., Morgan, B.J.T., and Catchpole, E.A., 2002, Integrating mark-recapturerecovery and census data to estimate animal abundance and demographic parameters: Biometrics, v. 58, p. 540-547.

Beston, J.A., 2011, Variation in life history and demography of the American black bear: Journal of Wildlife Management, v. 75, p. 1588-1596.

Beyer, H.L., 2012, Geospatial modelling environment (vers. 0.7.2.1), accessed June 1, 2013, at http://www.spatialecology.com/gme.

Boersen, M.R., Clark, J.D., and King, T.L. 2003, Estimating black bear population density and genetic diversity at Tensas River, Louisiana using microsatellite DNA markers: Wildlife Society Bulletin, v. 31, p. 197-207.

Bowman, J.L., 1999, An assessment of habitat suitability and human attitudes for black bear restoration in Mississippi: Starkville, Miss., Mississippi State University, Ph.D. dissertation, 345 p.

Bowman, J.L., Leopold, B.D., Vilella, F.J., Gill, D.A., and Jacobson, H.A., 2001, Attitudes of landowners toward American black bears compared between areas of high and low bear populations: Ursus, v. 12, p. 153-160.

Breen, M., Jouquand, S., Renier, C., Mellersh, C.S., Hitte, C., Holmes, N.G., Cheron, A., Suter, N., Vignaux, F., Bristow, A.E., Priat, C., McCann, E., Andre, C., Boundy, S., Gitsham, P., Thomas, R., Bridge, W.L., Spriggs, H.F., Ryder, E.J., Curson, A., Sampson, J., Ostrander, E.A., Binns, M.M., and Galibert, F., 2001, Chromosome-specific single-locus FISH probes allow anchorage of an 1800marker integratedradiation-hybrid/linkage map of the domestic dog genome to all chromosomes: Genome Research, v.11, p. 1784-1795.

Brook, B.W., O’Grady, J.J., Chapman, A.P., Burgman, M.A., Skcakaya, H.R., Frankham, R., 2000, Predictive accuracy of population viability analysis in conservation biology: Nature, v. 404, p. 385387.

Brooks, S.P., King, R., and Morgan, B.J.T., 2004. A Bayesian approach to combining animal abundance and demographic data: Animal Biodiversity and Conservation, v. 27, p. 515-529.

Bunnell, F.L., and Tait, D.E., 1981, Population dynamics of bears - implications, in Fowler, C.W., and Smith, T.D., eds. Dynamics of large mammal populations: New York, John Wiley and Sons, p.75-98.

Burnham, K.P., and Anderson, D.R., 2002, Model selection and multimodel inference: a practical information-theoretic approach: New York, Springer-Verlag, 488 p.

Burnham, K.P., and White, G.C., 2002, Evaluation of some random effects methodology applicable to bird ringing data: Journal of Applied Statistics, v. 29, p. 245-264.

Cagnacci, F., Boitani, L., Powell, R.A., and Boyce, M.S., 2010, Animal ecology meets GPS-based radiotelemetry: a perfect storm of opportunities and challenges: Philosophical Transactions of the Royal Society B: Biological Sciences , v. 365, p. 2157-2162.

Caswell, H., 2001, Matrix population models: construction, analysis, and interpretation (2d ed.): Sunderland, Mass., Sinauer Association, 722 p. 
Chetkiewicz, C.-L.B., and Boyce, M.S., 2009, Use of resource selection functions to identify conservation corridors: Journal of Applied Ecology, v. 46, p. 1036-1047.

Chetkiewicz, C.-L.B., St. Clair, C.C., and Boyce, M.S., 2006, Corridors for conservation: integrating pattern and process: Annual Review of Ecology, Evolution, and Systematics, v. 37, p. 317-342.

Clark, J.D., and Eastridge, R., 2006, Growth and sustainability of black bears at White River National Wildlife Refuge, Arkansas: Journal of Wildlife Management, v. 70, p. 1094-1101.

Clark, J.D., Eastridge, R., and Hooker, M.J., 2010, Effects of exploitation on black bear populations at White River National Wildlife Refuge: Journal of Wildlife Management, v. 74, p. 1448-1456.

Clark, J.D., Huber, D., and Servheen, C., 2002, Bear reintroductions: lessons and challenges: Ursus, v. 13, p. 335-345.

Clark, J.D., and Smith, K.G., 1994, A demographic comparison of 2 black bear populations in the Interior Highlands of Arkansas: Wildlife Society Bulletin, v. 22, p. 593-603.

Compton, B.W., Rhymer, J.M., and McCollough, M., 2002, Habitat selection by wood turtles (Clemmys insculpta): an application of paired logistic regression: Ecology, v. 83, p. 833-843.

Costello, C.M., Creel, S.R., Kalinowski, S.T., Vu, N.V., and Quigley, H.B., 2008, Sex-biased natal dispersal and inbreeding avoidance in American black bears as revealed by spatial genetic analyses: Molecular Ecology, v. 17, p. 4713-4723.

Coull, B.A., and Agresti, A., 1999, The use of mixed logit models to reflect heterogeneity in capturerecapture studies: Biometrics, v. 55, p. 294-301.

Courchamp, F., Berec, J., and Gascoigne, J., 2008, Allee effects in ecology and conservation: Oxford, U.K., Oxford University Press, 272 p.

Csiki, I., Lam, C., Key, A., Coulter, E., Clark, J.D., Pace, R.M., III, Smith, K.G., and Rhoads, D.D., 2003, Genetic variation in black bears in Arkansas and Louisiana using microsatellite DNA markers: Journal of Mammalogy, v. 84, p. 691-701.

Crook, A.C., and Chamberlain, M.J., 2010, A multiscale assessment of den selection by black bears in Louisiana: Journal of Wildlife Management, v. 74, p. 1639-1647.

Cushman, S.A., Schwartz, M.K., Hayden, J., and McKelvey, K.S., 2006, Gene flow in complex landscapes: testing multiple hypotheses with causal modeling: American Naturalist, v. 168, p. 486-499.

Czetwertynski, S.M., Boyce, M.S., and Schmiegelow, F.K., 2007, Effects of hunting on demographic parameters of American black bears: Ursus, v. 18, p. 1-18.

Derocher, A.E., and Taylor, M., 1994, Density-dependent population regulation of polar bears, in Taylor, M., ed., Density-dependent population regulation in black, brown, and polar bears:

International Conference on Bear Research and Management Monograph Series No. 3, p. 25-30.

Dixon, J.D., Oli, M.K., Wooten, M.C., Eason, T.H., McCown, J.W., and Paetkau, D., 2006, Effectiveness of a regional corridor in connecting two Florida black bear populations: Conservation Biology, v. 20, p. 155-162.

Dobey, S., Masters, D.V., Scheick, B.K., Clark, J.D., Pelton, M.R., and Sunquist, M., 2005, Population ecology of black bears in the Okefenokee-Osceola ecosystem: Wildlife Monographs, Number 158.

Dorazio, R.M., and Royle, J.A., 2003, Mixture models for estimating the size of a closed population when capture rates vary among individuals: Biometrics, v. 59, p. 351-364.

Duchesne, T., Fortin, D., and Courbin, N., 2010, Mixed conditional logistic regression for habitat selection studies: Journal of Animal Ecology 79, p. 5488-5555.

Duke, D.L., Hebblewhite, M., Paquet, P.C., Callaghan, C., and Percy, M., 2001, Restoring a largecarnivore corridor in Banff National Park, in Maehr, D.S., Noss, R., and Larkin, J.L., eds., Large 
mammal restoration: ecological and sociological challenges in the 21st century: Washington, D.C., Island Press, p. 261-275.

Eastridge, R., and Clark, J.D., 2001, Evaluation of 2 soft-release techniques to reintroduce black bears: Wildlife Society Bulletin, v. 29, p. 1163-1174.

Eberhardt, L.L., 1977, Optimal policies for conservation of large mammals, with special reference to marine ecosystems: Environmental Conservation, v. 4, p. 205-212.

Elowe, K.D., and Dodge,W.E., 1989, Factors affecting black bear reproductive success and cub survival: Journal of Wildlife Management, v. 53, p. 962-968.

Ennis, S., and Gallagher, T.F., 1994, A PCR-based sex-determination assay in cattle based on the bovine amelogenin locus: Animal Genetics, v. 25, p. 425-427.

Evanno, G., Regnaut, S., and Goudet, J., 2005, Detecting the number of clusters of individuals using the software STRUCTURE: a simulation study: Molecular Ecology, v. 14, p. 2611-2620.

Fisher, N.I., 1995, Statistical analysis of circular data: Cambridge, U.K., Cambridge University Press, 296 p.

Fisher, R.A., 1930, The genetical theory of natural selection: Oxford, U.K., Clarendon Press, 272 p.

Forester, J.D., Im, H.K., and Rathouz, P.J. 2009, Accounting for animal movement in estimation of resource selection functions: sampling and data analysis: Ecology, v. 90, p. 3554-3565.

Fortin, D., Beyer, H.L., Boyce, M.S., Smith, D.W., Duchesne, T., and Mao, J.S., 2005, Wolves influence elk movements: behavior shapes a trophic cascade in Yellowstone National Park: Ecology, v. 86, p.1320-1330.

Fowler, C.W., 1981, Density dependence as related to life history strategy: Ecology, v. 62, p. 602-610.

Fredholm, M., and Winterø, A.K., 1995, Variation of short tandem repeats within and between species belonging to the Canidae family: Mammalian Genome, v. 6, p. 11-18.

Fry, J., Xian, G., Jin, S., Dewitz, J., Homer, C., Yang, L., Barnes, C., Herold, N., and Wickham, J., 2011, Completion of the 2006 National Land Cover Database for the conterminous United States: Photogrammetric Engineering and Remote Sensing, v. 77, p. 858-864.

Garrison, E.P., McCown, J.W., and Oli, M.K., 2007, Reproductive ecology and cub survival of Florida black bears: Journal of Wildlife Management, v. 71, p. 720-727.

Garshelis, D.L., 1994, Density-dependent population regulation of black bears, in Taylor, M., ed., Density-dependent population regulation in black, brown, and polar bears: International Conference on Bear Research and Management Monograph Series No. 3., p. 3-14.

Ginzburg, L.R., Ferson, S., and Akcakaya, H.R., 1990, Reconstructibility of density dependence and the conservative assessment of extinction risk: Conservation Biology, v. 4, p. 63-70.

Goodman, D., 2002, Predictive Bayesian population viability analysis: a logic for listing criteria, delisting criteria, and recovery plans, in Beissinger, S.R., and McCullough, D.R., eds., Population viability analysis: Chicago, Ill., The University of Chicago Press, p. 447-469.

Haines-Young, R., and Chopping, M., 1996, Quantifying landscape structure: a review of landscape indices and their application to forested landscapes: Progress in Physical Geography, v. 20, p. 418445.

Hanski, I., 1996, Metapopulation ecology, in Rhodes, O.E., Jr., Chesser, R.K., and Smith, M.H., eds., Population dynamics in ecological space and time: Chicago, Ill., The University of Chicago Press, p. 13-43.

Haroldson, M.A., Schwartz, C.C., and White, G.C., 2006, Survival of independent grizzly bears in the Greater Yellowstone Ecosystem, 1983-2001, in Schwartz, C.C., Haroldson, M.A., White, G.C., Harris, R.B., Cherry, S., Keating, K.A., Moody, D., and Servheen, C., Temporal, spatial, and 
environmental influences on the demographics of grizzly bears in the Greater Yellowstone Ecosystem: Wildlife Monographs 161, p. 33-42.

Harrison, S., and Quinn, J.F., 1989, Correlated environments and the persistence of metapopulations: Oikos, v. 56, p. 293-298.

Heino, M., Kaitala, V., Ranta, E., and Lindstrom, J., 1997, Synchronous dynamics and rates of extinction in spatially structured populations: Proceedings of the Royal Society B-Biological Sciences, v. 264, p. 481-486.

Heisey, D. M., and Fuller, T.K., 1985, Evaluation of survival and cause-specific mortality rates using telemetry data: Journal of Wildlife Management, v. 49, p. 668-674.

Hellgren, E.C., 1988, Ecology and physiology of a black bear (Ursus americanus) population in Great Dismal Swamp and reproductive physiology in the captive female black bear: Blacksburg, Va., Virginia Polytech Institute and State University, Ph.D. Dissertation, 231 p.

Hellgren, E.C., and Vaughan, M.R., 1994, Conservation and management of isolated black bear populations in the southeastern coastal plain of the United States: Proceedings of the Annual Conference of the Southeastern Association of Fish and Wildlife Agencies, v. 48, p. 276-285.

Hellgren, E.C., Onorato, D.P., and Skiles, J.R., 2005, Dynamics of a black bear population within a desert metapopulation: Biological Conservation, v. 122, p. 131-140.

Hightower, D.A., 2003, Fine-scaled movements and habitat use of black bears in south central Louisiana: Baton Rouge, La., Louisiana State University, Master of Science Thesis, 79 p.

Hightower, D.A., Wagner, R.O., and Pace, R.M., III, 2002, Denning ecology of female American black bears in south central Louisiana: Ursus, v. 13, p. 11-18.

Hood, G.M., 2010, PopTools (vers. 3.2.5), accessed June 1, 2013, at http://www.poptools.org. Hooker, M.J., 2010, Estimating population parameters of the Louisiana black bear in the Tensas River Basin, Louisiana, using robust design capture-mark-recapture: Knoxville, Tenn., University of Tennessee, Master of Science Thesis, $60 \mathrm{p}$.

Hosmer, D.W., Jr., and Lemeshow, S., 1989, Applied logistic regression: New York, John Wiley and Sons, $392 \mathrm{p}$.

Kendall, K.C., Stetz, J.B., Boulanger, J., Macleod, A.C., Paetkau, D., and White, G.C., 2009, Demography and genetic structure of a recovering grizzly bear population: Journal of Wildlife Management, v. 73, p. 3-17.

Kennedy, M.L., Kennedy, P.K., Bogan, M.A., and Waits, J.L., 2002, Geographic variation in the black bear (Ursus americanus) in the eastern United States and Canada: Southwestern Naturalist, v. 47, p. $257-266$.

Kéry, M., and Schaub, M., 2012, Bayesian population analysis using WinBUGS: a hierarchical perspective: Amsterdam, Netherlands, Academic Press, 560 p.

Kitahara, E., Isagi, Y., Ishibashi, Y., and Saitoh, T., 2000, Polymorphic microsatellite DNA markers in the Asiatic black bear Ursus thibetanus: Molecular Ecology, v. 9, p. 1661-1662.

Lande, R., 1987, Extinction thresholds in demographic models of territorial populations: The American Naturalist, v. 130, p. 624-635.

Lande, R., 1993, Risks of population extinction from demographic and environmental stochasticity and random catastrophes: The American Naturalist, v. 142, p. 911-927.

Latham, A.D.M., Latham, M.C., Boyce, M.S., and Boutin, S., 2011, Movement responses by wolves to industrial linear features and their effect on woodland caribou in northeastern Alberta: Ecological Applications, v. 21, p. 2854-2865. 
Laufenberg, J.S., 2014, Population dynamics and genetic structure of Louisiana black bears in the Lower Mississippi Alluvial Valley of Louisiana: Knoxville, Tn., University of Tennessee, Ph.D. Dissertation, 224 p.

Laufenberg, J.S., van Manen, F.T., and Clark, J.D., 2013, Effects of sampling conditions on DNA-based estimates of American black bear abundance: Journal of Wildlife Management, v. 77, p. 1010-1020.

Lebreton, J.-D., and Gimenez, O., 2013, Detecting and estimating density dependence in wildlife populations: Journal of Wildlife Management, v. 77, p. 12-23.

Lee, D.J., and Vaughan, M.R., 2003, Dispersal movements by subadult American black bears in Virginia: Ursus, v. 14, no. 2, p. 162-170.

Lewis, J.S., Rachlow, J.L., Garton, E.O., Vierling, L.A., 2007, Effects of habitat on GPS collar performance: using data screening to reduce location error: Journal of Applied Ecology, v. 44, v. 663-671.

Lindzey, J.S., Alt, G.L., McLaughlin, C.R., and Kordek, W.S., 1983, Population response of Pennsylvania black bears to hunting: Bears-Their Biology and Management, p. 34-39.

Link, W.A., 2003, Nonidentifiability of population size from capture-recapture data with heterogeneous detection probabilities: Biometrics, v. 59, p. 1123-1130.

Link, W.A., and Barker, R.J., 2005, Modeling association among demographic parameters in analysis of open population capture-recapture data: Biometrics, v. 61, p. 46-54.

Link, W.A., and Barker, R.J., 2010, Bayesian inference with ecological applications: Burlington, Mass., Academic Press, 339 p.

Lombardo, C. A. 1993. The population ecology of black bears on Camp LeJeune, North Carolina:

Knoxville, Tenn., University of Tennessee, Master of Science Thesis, 155 p.

Lowe, C.L., 2011, Estimating population parameters of the Louisiana black bear in the Upper Atchafalaya River Basin: Knoxville, Tenn., University of Tennessee, Master of Science Thesis, 60 p.

MacArthur, R.H., and Wilson, E.O., 1967, The theory of island biogeography: Princeton, N.J., Princeton University Press, 203 p.

Maddrey, R.C., 1995. Morphology, reproduction, food habits, crop depredation, and mortality of black bears on the Neuse-Pamlico Peninsula, North Carolina: Knoxville, Tenn., University of Tennessee, Master of Science Thesis, 149 p.

Manel, S., Gaggiotti, O.E., and Waples, R.S., 2005, Assignment methods: matching biological questions with appropriate techniques: Trends in Ecology \& Evolution, v. 20, p. 136-142.

Manly, B.F.J., McDonald, L.L., Thomas, D.L., McDonald, T.L., and Erickson, W.P. 2002, Resource selection by animals: statistical design and analysis for field studies (2d ed.): Dordrecht, Netherlands, Kluwer Academic Publishers, 240 p.

Marchinton, F.B., 1995, Movement ecology of black bears in a fragmented bottomland hardwood habitat in Louisiana: Knoxville, Tenn., University of Tennessee, Master of Science Thesis, 214 p.

McCullough, D.R., 1996, Metapopulation management: what patch are we in and what corridor should we take?, in McCullough, D.R., ed., Metapopulations and wildlife conservation: Washington, D.C., Island Press, p. 405-410.

McDonald, T.L., 2013, The point process use-availability or presence-only likelihood and comments on analysis: Journal of Animal Ecology, v. 82, p. 1174-1182.

McGarigal, K., and Marks, B.J., 1995, FRAGSTATS: spatial pattern analysis program for quantifying landscape structure: Washington, D.C., U.S. Forest Service General Technical Report PNW-351, $122 \mathrm{p}$. 
McLellan, B., 1994, Density-dependent population regulation of brown bears, in Taylor, M., ed., Density-dependent population regulation in black, brown, and polar bears: International Conference on Bear Research and Management Monograph Series No. 3, p. 15-24.

Meredith, E.P., Rodzen, J.A., Banks, J.D., and Jones, K.C., 2009, Characterization of 29 tetranucleotide microsatellite loci in black bear for use in forensic and population applications: Conservation Genetics, v. 10, p. 693-696.

Miller, S.D., 1994, Black bear reproduction and cub survivorship in south-central Alaska: Bears-Their Biology and Management, v. 9, p. 263-273.

Miller, S.D., Sellers, R.A., and Keay, J.A., 2003, Effects of hunting on brown bear cub survival and litter size in Alaska: Ursus, v.14, p. 130-152.

Mills, L.S., 2007, Conservation of wildlife populations: Malden, Mass., Blackwell Publishing, 407 p.

Mills, L.S., and Allendorf, F.W., 1996, The one-migrant-per-generation rule in conservation and management: Conservation Biology, v.10, p. 1509-1518.

Mills, L.S., Hayes, S.G., Baldwin, C., Wisdom, M.J., Citta, J., Mattson, D.J., and Murphy, K., 1996, Factors leading to different viability predictions for a grizzly bear data set: Conservation Biology, v. 10, p. 863-873.

Morris, W.F., and Doak, D.F., 2002, Quantitative conservation biology: theory and preactice of population viability analysis: Sunderland, Mass., Sinauer Associates, Inc., 480 p.

Murrow, J.L., and Clark, J.D., 2012, Effects of hurricanes Katrina and Rita on Louisiana black bear habitat: Ursus, v. 2, no. 2, p. 192-205.

Murrow, J.L., Clark, J.D., Flanagan, S., and Thatcher, C., 2013, A tool for estimating effects of land-use changes on Louisiana black bear habitat. Final report to the Fish and Wildlife Service: Lafayette, La., Ecological Services Field Office, 61 p.

Neal, W.A., 1990, Proposed threatened status for the Louisiana black Bear: Federal Register, v. 55, no. 120, p. 25341-25345.

Nelson, J.G., Day, J.C., and Sportza, L.M., eds., 2003, Protected areas and the regional planning imperative in North America: integrating nature conservation and sustainable development: Calgary, Alberta, Canada, University of Calgary Press, 429 p.

Nielsen, S.E., Boyce, M.S., Stenhouse, G.B., and Munro, R.H.M., 2002, Modeling grizzly bear habitats in the Yellowhead Ecosystem of Alberta: taking autocorrelation seriously: Ursus, v. 13, p. 45-56.

Noss, R.F.. 1987, Protecting natural areas in fragmented landscapes: Natural Areas Journal, v. 7, p. 2-13.

Noss, R.F., 2003, A checklist for wildlands network designs: Conservation Biology, v. 17, p. $1270-1275$.

Nyland, P.D., 1995, Black bear habitat relationships in coastal Louisiana: Baton Rouge, La., Louisiana State University, Master of Science Thesis, 76 p.

Obbard, M.E., and Howe, E.J., 2008, Demography of black bears in hunted and unhunted areas of the boreal forest of Ontario: Journal of Wildlife Management, v. 72, p. 869-880.

O’Connell, K.C., 2013, Population dynamics of the Louisiana black bear in the Upper Atchafalaya River Basin: Knoxville, Tenn., University of Tennessee, Master of Science Thesis, 63 p.

O’Connell, K.C., Lowe, C.L., and Clark. J.D., 2014, Effects of opening the Morganza Flood Control Structure on a threatened black bear population in Louisiana: Animal Conservation, v. 17, p. 476-485.

Onorato, D.P., and Hellgren, E.C., 2001, Black bear at the border: natural recolonization of the TransPecos, in Maehr, D.S., Noss, R. and Larkin, J.L., eds., Large mammal restoration: ecological and sociological challenges in the 21st century: Washington, D.C., Island Press, 245-259 p. 
Otis, D.L., Burnham, K.P., White, G.C., and Anderson, D.R., 1978, Statistical inference from capture data on closed animal populations: Wildlife Monographs, v. 62, xx p.

Pace, R.M., III, Anderson, D.R., and Shively, S., 2000, Sources and patterns of black bear mortality in Louisiana: Proceedings of the Annual Conference of the Southeast Association of Fish and Wildlife Agencies, v. 54, no. 365-373.

Paetkau, D., 2003, An empirical exploration of data quality in DNA-based population inventories: Molecular Ecology, v. 12, p. 1375-1387.

Paetkau, D., Calvert, W., Stirling, I., and Strobeck, C., 1995, Microsatellite analysis of population structure in Canadian polar bears: Molecular Ecology, v. 4, p. 347-354.

Paetkau, D., Slade, R., Burden, M., and Estoup, A., 2004, Genetic assignment methods for the direct, real-time estimation of migration rate: a simulation-based exploration of accuracy and power:

Molecular Ecology, v. 13, p. 55-65.

Paetkau, D., and Strobeck, C., 1994, Microsatellite analysis of genetic variation in black bear populations: Molecular Ecology, v. 3, p. 489-495.

Palmqvist, E., and Lundberg, P., 1998, Population extinctions in correlated environments: Oikos, v. 83, p. 359-367.

Pelton, M.R., 1982, Black bear, in Chapman, J.A., and Feldhammer, G.A., eds., Wild mammals of North American: biology, management and economics: Baltimore, Md., The John Hopkins University Press, p. 504-514.

Pelton, M.R., 2003, Black bear, in Feldhamer, G.A., Thompson, B.C., and Chapman, J.A., eds., Wild mammals of North America: Baltimore, Md., The Johns Hopkins University Press, p. 547-55.

Piry, S., Alapetite, A., Cornuet, J.-M.,Paetkau, D., Baudouin, L., and Estoup, A., 2004. GeneClass2: a software for genetic assignment and first-generation migrant detection: Journal of Heredity, v. 95, p. 536-539.

Pledger, S., 2000, Unified maximum likelihood estimates for closed capture-recapture models using mixtures: Biometrics, v. 56, p. 434-442.

Plummer, M.N., 2011, rjags: Bayesian graphical models using mcmc. R package version 3-8.

Plummer, M., Best, N., Cowles, K., and Vines, K., 2006, CODA: convergence diagnosis and output analysis for MCMC: R News, v. 6, p. 7-11.

Pollock, K.H., 1982, A capture-recapture design robust to unequal probability of capture: Journal of Wildlife Management, v. 46, p. 757-760.

Pollock, K.H., Winterstein, S.R., Bunck, C.M., and Curtis, P.D., 1989, Survival analysis in telemetry studies: the staggered entry design: Journal of Wildlife Management, v. 53, p. 7-15.

Pritchard, J.K., Stephens, M., and Donnelly, P., 2000, Inference of population structure using multilocus genotypes data: Genetics, v. 155, p. 945-959.

Proctor, M., McLellan, B.N., and Strobeck, C., 2002, Population fragmentation of grizzly bears in southeastern British Columbia, Canada: Ursus, v. 13, p. 153-160.

Proctor, M.F., McLellan, B.N., Strobeck, C., and Barclay, R.M.R., 2004, Gender-specific dispersal distances of grizzly bears estimated by genetic analyses: Canadian Journal of Zoology, v. 82, p. 1108-1118.

Proctor, M.F., McLellan, B.N., Strobeck, C., and Barclay, R.M.R., 2005, Genetic analysis reveals demographic fragmentation of grizzly bears yielding vulnerability by small populations: Proceedings of the Royal Society B: Biological Sciences, v. 272, p. 2409-2416.

R Core Team, 2013, R: a language and environment for statistical computing: Vienna, Austria, R Foundation for Statistical Computing. [Also available at http://www.R-project.org/.] 
Raymond, M., and Rousset, F., 1995, GENEPOP (Version 1.2) - Population genetics software for exact tests and ecumenicism: Journal of Heredity, v. 86, p. 248-249.

Rhodes, J.R., MaAlpine, C.A., Lunney, D., and Possingham,H.P., 2005, A spatially explicit habitat selection model incorporating home range behavior: Ecology, v. 86, p.1999-1205.

Rogers, L.L., 1987, Factors influencing dispersal in the black bear, in Chepko-Sade, B.D., and Halpin, Z.T., eds., Mammalian dispersal patterns, the effects of social structure on population genetics: Chichago Ill., University of Chicago Press, p. 75-84.

Rogers, M.J.,1973, Movements and reproductive success of black bears introduced into Arkansas: Proceedings of the Southeastern Association of Fish and Wildlife Agencies, v. 27, p. 307-308.

Roon, D.A., Waits, L.P., and Kendall, K.C., 2005, A simulation test of the effectiveness of several methods for error-checking non-invasive genetic data: Animal Conservation, v. 8, p. 203-215.

Royle, J.A., Dorazio, R.M., and Link, W.A., 2007, Analysis of multinomial models with unknown index using data augmentation: Journal of Computational and Graphical Statistics, v. 16, p. 67-85.

Royle, J.A., and Dorazio, R.M., 2008, Hierarchical modeling and inference in ecology: the analysis of data from populations, metapopulations and communities: New York, Academic Press, 444 p.

Royle, J.A., and Dorazio, R.M., 2012, Parameter-expanded data augmentation for Bayesian analysis of capture-recapture models: Journal of Ornithology, v. 152, Supplement 2, p. S521-S537.

St. Amant, L.S., 1959, Louisiana wildlife inventory and management plan: New Orleans, La., Louisiana Wild Life and Fisheries Commission, 329 p.

Sargeant, G.A., and Ruff, R.L., 2001, Demographic response of black bears at Cold Lake, Alberta, to the removal of adult males: Ursus, v. 12, p. 59-68.

Schaub, M., Gimenez, O., Sierro, A., and Arlettaz, R., 2007, Use of integrated modeling to enhance estimates of population dynamics obtained from limited data: Conservation Biology, v. 21, p. 945-955.

Scheick, B.K., and McCown, W., 2014, Geographic distribution of American black bears in North America: Ursus, v. 25, p. 24-33.

Schwartz, C.C., and Franzmann, A.W., 1992, Dispersal and survival of subadult black bears from the Kenai Peninsula, Alaska: Journal of Wildlife Management, v. 56, p. 426-431.

Schwartz, C.C., Haroldson, M.A., and White, G.C., 2006, Survival of cub and yearling grizzly bears in the Greater Yellostone Ecosystem, 1983-2001, in Schwartz, C.C., Haroldson, M.A., White, G.C., Harris, R.B., Cherry, S., Keating, K.A., Moody, D.S., and Servheen, C., eds., Temporal, spatial, and environmental influences on the demographics of grizzly bears in the Greater Yellowstone Ecosystem: Wildlife Monographs 161, p. 18-23.

Schwartz, C.C., and White, G.C., 2008, Estimating reproductive rates for female bears: proportions versus transition probabilities: Ursus, v. 19, p. 1-12.

Settlage, K., Svan Manen, F.T., Clark, J.D., and King, T.L., 2008, Challenges of DNA-based markrecapture studies of American black bears: Journal of Wildlife Management, v. 72, p. 1035-1042.

Shaffer, M., 1987, Minimum viable populations: coping with uncertainty, in Soule, M.E., ed., Viable populations for conservation: Cambridge, UK, Cambridge University Press, p. 69-86.

She, J.X., Autem, M., Kotulas, G., Pasteur, N., and Bonhomme, F., 1987, Multivariate analysis of genetic exchanges between Solea aegyptiaca and Solea senegalensis (Teleosts, Soleidae): Biological Journal of the Linnean Society, v. 32, p. 357-371.

Simek, S.L., Belant, J.L., Young, B.W., Shropshire, C., and Leopold, B.D., 2012, History and status of the American black bear in Mississippi: Ursus, v. 23, p. 159-167.

Smith, T.R., and Pelton, M.R., 1990, Home ranges and movements of black bears in a bottomland hardwood forest in Arkansas: Ursus, v. 8, p. 213-218. 
Sokal, R.R., and Rohlf, F.J., 1995, Biometry (3d ed.): San Franciso, Calif., W. H. Freeman and Company, $887 \mathrm{p}$.

Spear, S.F., Balkenhol, N., Fortin, M., McRae, B.H., and Scribner, K., 2010 Use of resistance surfaces for landscape genetic studies: considerations for parameterization and analysis: Molecular Ecology, v. 19, p. 3576-3591.

Stinson, P.H., 1996, Black bears in the Tunica Hills: present status and habitat suitability: Baton Rouge, La., Louisiana State University, Master of Science Thesis, 77 p.

Storfer, A., Murphy, M.A., Spear, S.F., Holderegger, R., and Waits, L.P., 2010, Landscape genetics: where are we now?: Molecular Ecology, v. 19, p. 3496-3514.

Taberlet, P., Camarra, J.-J.,Griffin, S., Uhrès, E., Hanotte, O., Waits, L.P., Dubois-Paganon, C., Burke, T., and Bouvet, J., 1997, Non-invasive genetic tracking of the endangered Pyrenean brown bear population: Molecular Ecology, v. 6, p. 869-876.

Taberlet, P., and Luikart, G., 1999, Non-invasive genetic sampling and individual identification: Biological Journal of the Linnean Society, v. 68, p. 41-55.

Taylor, B.L., 1995, The reliability of using population viability analysis for risk classification of species: Conservation Biology, v. 9, p. 551-558.

Taylor, E.F., 1971, A radio-telemetry study of the black bear (Euarctos americanus) with notes on its history and present status in Louisiana: Baton Rouge, La., Louisiana State University, Master of Science Thesis, $87 \mathrm{p}$.

Taylor, M.K., Obbard, M., Pond, B., Kuc, M., and Abraham, D., 2006, RISKMAN: Stochastic and deterministic population modeling RISK MANagement decision tool for harvested and unharvested populations: Iqaluit, Nunavit Territory, Canada, Government of Nunavut, 58 p.

Therneau, T., 2013, A package for survival analysis in S. R package version 2.37-4, accessed March 1, 2013, at http://CRAN.R-project.org/package=survival.

Tredick, C.A., Vaughan, M.R., Stauffer, D.F., Simek, S.L., and Eason, T., 2007, Sub-sampling genetic data to estimate black bear population size: a case study: Ursus, v. 18, p. 179-188.

Triant, D.A., Pace, R.M., III, and Stine, M., 2004, Abundance, genetic diversity and conservation of Louisiana black bears (Ursus americanus luteolus) as detected through noninvasive sampling: Conservation Genetics, v. 5, p. 647-659.

Troxler, J.C., 2013, Population demographics and genetic structure of black bears in coastal Louisiana: Knoxville, Tenn., University of Tennessee, Master of Science Thesis, 90 p.

Turchin, P., 1998, Quantitative analysis of movement: measuring and modeling population redistribution in plants and animals: Sunderland, Mass., Sinauer Associates, 396 p.

Turner, M.G., Gardner, R.H., and O’Neill, R.V., 2001, Landscape ecology in theory and practice: pattern and process: New York, Springer-Verlag, 406 p.

U.S. Bureau of the Census, 2012, Census 2010 TIGER/Line Data, accessed March 1, 2013, at http://www.census.gov/geo/www/tiger/tgrshp2010/tgrshp2010.html.

U.S. Bureau of the Census, 2009, Census 2000 TIGER/Line Data, accessed February1, 2013, at http://www.esri.com/data/download/census2000_tigerline/index.html.

U.S. Fish and Wildlife Service (USFWS), 1992, Endangered and threatened wildlife and plants; determination for threatened status for U. a. luteolus (Louisiana black bear): Federal Register, v. 57, p. 588-595.

U.S. Fish and Wildlife Service, 1995, Louisiana black bear recovery plan: Jackson, Miss., U.S. Fish and Wildlife Service, 52 p. 
U.S. Fish and Wildlife Service, 2009, Endangered and threatened wildlife and plants; designation of critical habitat for the Louisiana black bear (Ursus americanus luteolus): Federal Register, v. 74, p. 10350-10409.

Van Why, K.R., and Chamberlain, M.J., 2003a, Sportsmen knowledge and opinions on restoring the Louisiana black bear: Proceedings of the Southeastern Association of Fish and Wildlife Agencies, v. 57, p. 299-307.

Van Why, K.R., and Chamberlain, M.J., 2003b, Mortality of Louisiana black bears associated with elevated train trestles: Canadian Field-Naturalist, v. 117, p. 113-115.

Wade, P.R., 2002, Bayesian population viability analysis, in Beissinger, S.R., and McCullough, D.R., eds., Population viability analysis: Chicago, Ill., The Chicago University Press, p. 213-238.

Wagner, R.O., 1995, Movement patterns of black bears in south central Louisiana: Baton Rouge, La., Louisiana State University, Master of Science Thesis, 57 p.

Wagner, R.O., Hightower, D.A., and Pace, R.M., III, 2001, Measuring levels and patterns of activity in black bears: Ursus, v. 12, p. 181-188.

Waits, L.P., Luikart, G., and Taberlet, P., 2001, Estimating the probability of identity among genotypes in natural populations: cautions and guidelines: Molecular Ecology, v. 10, p. 249-256.

Waller, B.W., Belant, J.L., Young, B.W., Leopold, B.D., and Simek, S.L., 2012, Denning chronology and den characteristics of American black bears in Mississippi: Ursus, v. 23, p. 6-11.

Warrilow, J., Culver, M., Hallerman, E., and Vaughan, M., 2001, Subspecific affinity of black bears in the White River National Wildlife Refuge: Journal of Heredity, v. 92, p. 226-233.

Wear, B.J., Eastridge, R., and Clark, J.D., 2005, Factors affecting settling, survival, and viability of black bears reintroduced to Felsenthal National Wildlife Refuge, Arkansas: Wildlife Society Bulletin, v. 33, p. 1363-1374.

Weaver, K.M., 1990, The ecology and management of black bears in the Tensas River Basin of Louisiana: Knoxville, Tenn., University of Tennessee, Ph.D. dissertation, 654 p.

Weaver, K.M., and Pelton, M.R., 1994, Denning ecology of black bears in the Tensas River Basin of Louisiana: Proceedings of the International Conference on Bear Research and Management, v. 9, p. $427-433$.

White, G.C., 2000, Population viability analysis: data requirements and essential analyses, in Boitani, L., and Fuller, T.K., eds. Research techniques in Animal Ecology: New York, Columbia University Press, p. 288-331.

White, G.C., Burnham, K.P., and Barker, R.J., 2009, Evaluation of a Bayesian MCMC random effects inference methodology for capture-mark-recapture data, in Thomson, D.L., Cooch, E.G., and Conroy, M.J., ed., Modeling demographic processes in marked populations: New York, Springer, p. 1119-1127.

Willey, C.H., 1974, Aging black bears from first premolar tooth sections: Journal of Wildlife Management, v. 38, p. 97-100.

Wooding, J.B., Cox, J.A., and Pelton, M.R., 1994, Distribution of black bears in the southeastern coastal plain: Proceedings of the Annual Conference of the Southeastern Association of Fish and Wildlife Agencies, v. 48, p. 270-275.

Woods, J.G., Paetkau, D., Lewis, D., McLellan, B.N., Proctor, M., and Strobeck, C., 1999, Genetic tagging of free-ranging black and brown bears: Wildlife Society Bulletin, v. 27, p. 616-627.

Wright, S., 1931, Evolution in Mendelian populations: Genetics, v. 16, p. 97-159.

Wright, S., 1943, Isolation by distance: Genetics, v. 28, p. 114-138. 
ISSN (online)

http://dx.doi.org/10.3133/ofr20141228 